\title{
Measurement of the Ratio of Branching Fractions $\operatorname{Br}\left(B_{s}^{0} \rightarrow D_{s}^{-} \pi^{+}\right) / B r\left(B^{0} \rightarrow D^{-} \pi^{+}\right)$at CDF-II \\ by \\ Ivan Krešimir Furić
}

Submitted to the Department of Physics

in partial fulfillment of the requirements for the degree of

Doctor of Philosophy

at the

\section{MASSACHUSETTS INSTITUTE OF TECHNOLOGY}

March 2004

(C) Ivan Krešimir Furić, MMIV. All rights reserved.

The author hereby grants to MIT permission to reproduce and distribute publicly paper and electronic copies of this thesis document in whole or in part.

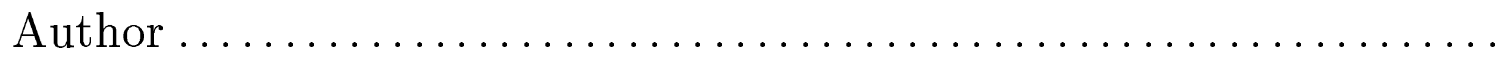

Department of Physics

March 12, 2004

Certified by .

Christoph M. E. Paus Associate Professor

Thesis Supervisor

Accepted by

Thomas J. Greytak Chairman, Department Committee on Graduate Students 


\title{
Measurement of the Ratio of Branching Fractions $\operatorname{Br}\left(B_{s}^{0} \rightarrow D_{s}^{-} \pi^{+}\right) / B r\left(B^{0} \rightarrow D^{-} \pi^{+}\right)$at CDF-II \\ by
}

\section{Ivan Krešimir Furić}

\author{
Submitted to the Department of Physics \\ on March 12, 2004, in partial fulfillment of the \\ requirements for the degree of \\ Doctor of Philosophy
}

\begin{abstract}
The measurement of $B_{s}^{0}$ mixing is one of the flagship analyses for the Run II B physics program. The sensitivity of the measurement to the frequency of $B_{s}^{0}$ oscillations strongly depends on the number of reconstructed $B_{s}^{0}$ mesons. We present the measurement of the ratio of branching fractions $\operatorname{Br}\left(B_{s}^{0} \rightarrow D_{s}^{-} \pi^{+}\right) / B r\left(B^{0} \rightarrow D^{-} \pi^{+}\right)$, which directly influences the number of $B_{s}^{0}$ events available for the measurement of $B_{s}^{0}$ mixing at CDF-II.

We analyze $115 \mathrm{pb}^{-1}$ of data collected with the CDF-II detector in $p \bar{p}$ collisions at $\sqrt{s}=1.96 \mathrm{TeV}$ using a novel displaced track trigger. We reconstruct $78 \pm 11$ $B_{s}^{0} \rightarrow D_{s}^{-} \pi^{+}$decays and $1153 \pm 45 B^{0} \rightarrow D^{-} \pi^{+}$decays with good signal to background ratio. This is the world's largest sample of fully reconstructed $B_{s}^{0} \rightarrow D_{s}^{-} \pi^{+}$decays. We find the ratio of production fractions multiplied by the ratio of branching fractions to be:

$$
\frac{f_{s}}{f_{d}} \cdot \frac{B r\left(B_{s}^{0} \rightarrow D_{s}^{-} \pi^{+}\right)}{B r\left(B^{0} \rightarrow D^{-} \pi^{+}\right)}=0.325 \pm 0.046(\text { stat }) \pm 0.034(\text { syst }) \pm 0.084(B R)
$$
\end{abstract}

Using the world average value of $f_{s} / f_{d}=0.26 \pm 0.03$, we infer that the ratio of branching fractions is:

$$
\frac{B r\left(B_{s}^{0} \rightarrow D_{s}^{-} \pi^{+}\right)}{B r\left(B^{0} \rightarrow D^{-} \pi^{+}\right)}=1.25 \pm 0.18(\text { stat }) \pm 0.13(\text { syst }) \pm 0.32(B R) \pm 0.14(P R)
$$

where the last uncertainty is due to the uncertainty on the world average measurement of the ratio of $B_{s}^{0}$ to $B^{0}$ production rates, $f_{s} / f_{d}$.

Thesis Supervisor: Christoph M. E. Paus

Title: Associate Professor 


\section{Acknowledgments}

I would like to thank my advisor Christoph Paus for his guidance and support. He exemplifies persistence, hard work and high standards. In particular, I thank him for always insisting that issues are fundamentally simple and all numbers have to make sense. This simple principle introduces clarity into problem solving and applies far beyond particle physics research.

I want to thank the entire MIT group at CDF for creating a stimulating environment to do work on hardware and analysis. I thank Ilya Kravchenko, Gerry Bauer, Jeff Tseng, Steve Tether and Sham Sumorok for sharing their knowledge and experience with me, and for always having simple solutions to what I considered to be difficult conundrums. I have learned a lot from working with Konstantin Anikeev, Andreas Korn, Mike Mulhearn and Sasha Rakitine these last four years. Every one of them provided their own unique input and approach to the project at hand, ranging from building the L3 farm, building the ToF detector to analyzing data and doing measurements. It was a pleasure doing analysis with both Alberto Belloni and Stefan Gromoll. I thank the younger students, Boris Iyutin, Nuno Leonardo, Arkadiy Bolshov and Jeff Miles, for taking over the hardware projects so that the seniors could graduate faster.

I would like to thank Heather Ray for her unconditional love and support. She has brought much happiness into my life.

I thank my family for always being there for me. I spent a lot of time as a child with my grandparents, Ivan and Marija Furić. I cherish the memories of those times, and regret that my grandfather can not be here with me to share this honor. I thank my uncle Krešimir Furić for infusing me with his passion for devices and tinkering, and I thank his family for their love.

Most of all, I am indebted to my parents, Elizabeta and Miroslav and my sister Vesna Furić. They worked selflessly on making me the person I am today since the day I was born. Whatever quickness and intuition I have, I got from my mother, while my patience and methodical approach to problems come from my father. I dedicate this thesis to the three of you. 


\section{Contents}

1 Introduction $\quad 21$

2 Theoretical Overview 23

2.1 The CKM Matrix . . . . . . . . . . . . . . 24

2.1.1 Wolfenstein Parametrization and the Bjorken Triangle . . . 26

2.2 Constraining the CKM Matrix . . . . . . . . . . 28

2.3 Considerations for Measuring $B_{s}^{0}$ Mixing . . . . . . . . . 31

2.4 Theory of Hadronic B Decays . . . . . . . . . . . . . 33

2.4.1 Factorization Approach . . . . . . . . . . 34

2.4.2 Heavy Quark Effective Theory and Symmetries . . . . . . 35

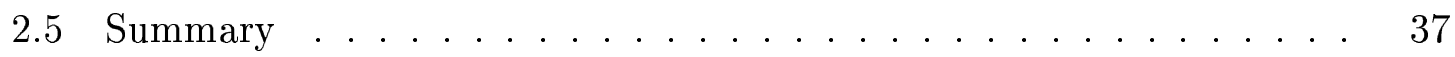

3 Experimental Apparatus $\quad 39$

3.1 Tevatron - the Source of $p \bar{p}$ Collisions . . . . . . . . . . . . 40

3.2 The CDF-II detector . . . . . . . . . . . . . . . 42

3.3 Standard Definitions in CDF-II . . . . . . . . . . . . 44

3.4 Tracking Systems . . . . . . . . . . . . . . 46

3.4.1 Silicon Tracking Detectors . . . . . . . . . . . 47

3.4.2 Central Outer Tracker . . . . . . . . . . . . 49

3.4.3 Pattern Recognition Algorithms . . . . . . . . . . 52

3.4.4 Momentum Scale ................ 53

3.5 Time of Flight . . . . . . . . . . . . . 55

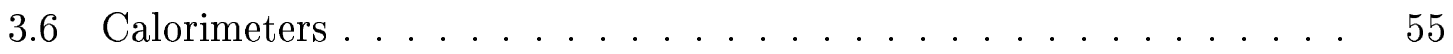


3.7 Muon Systems. . . . . . . . . . . . . . . . . . . 56

3.8 Triggering $\ldots \ldots \ldots \ldots \ldots \ldots \ldots \ldots \ldots \ldots \ldots \ldots$

3.8.1 Level 1 Trigger . . . . . . . . . . . . . . . . 61

3.8.2 Level 2 Trigger . . . . . . . . . . . . . . . 62

3.8.3 Level 3 Trigger . . . . . . . . . . . . . . . . . . 64

4 Dataset and Candidate Selection $\quad 67$

4.1 Good Runs . . . . . . . . . . . . . . . . . . . 68

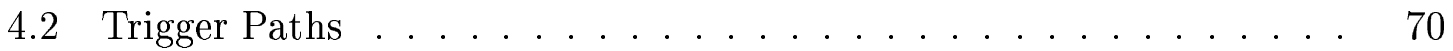

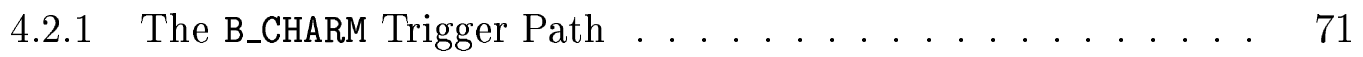

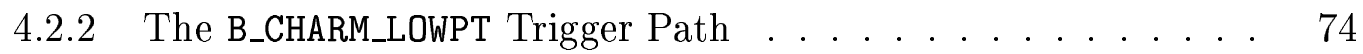

4.2.3 The B_CHARM_HIGHPT Trigger Path . . . . . . . . . . 75

4.2.4 Seven Track Trigger Paths . . . . . . . . . . . . 77

4.3 Event Selection . . . . . . . . . . . . . . . . . . 79

4.3.1 Track Preparation . . . . . . . . . . . . . . 79

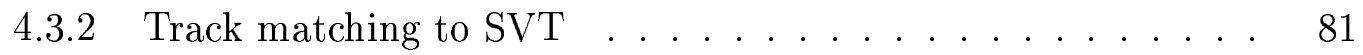

4.3 .3 Trigger Confirmation . . . . . . . . . . . . . . 82

4.3.4 Candidate Reconstruction . . . . . . . . . . . . 83

4.3.5 Optimization of Selection Requirements . . . . . . . . . . . 84

4.4 Summary . . . . . . . . . . . . . . . . . . 91

5 Rate Analysis $\quad 93$

5.1 Monte Carlo Simulation . . . . . . . . . . . . . . . 93

5.1.1 B Hadron Generation and Decay . . . . . . . . . . . . 94

5.1 .2 Realistic Simulation . . . . . . . . . . . . . 9 95

$5.2 \quad$ Fitting $B$ Mass Spectra . . . . . . . . . . . . . . . . 97

5.2 .1 General Fit Model . . . . . . . . . . . . . . . . . . . . 102

5.2.2 Fit Model: Signal and Cabibbo Suppressed Decays _. . . . 103

5.2 .3 Fit Model: $B^{+} \ldots \ldots \ldots \ldots$. . . . . . . . . . 104

5.2 .4 Fit Model: $B_{s} \ldots \ldots \ldots \ldots \ldots \ldots \ldots$

$5.2 .5 \quad$ Fit Model: $B^{0} \ldots \ldots \ldots \ldots \ldots \ldots$ 
$5.2 .6 \quad B$ Meson Yields . . . . . . . . . . . . . . . . . . . . 107

5.3 Monte Carlo Validation . . . . . . . . . . . . . . . . . . . . . 114

5.3.1 Reweighting the Monte Carlo . . . . . . . . . . . 135

5.4 Ratio of Efficiencies . . . . . . . . . . . . . . . . . 137

5.5 Ratio of Branching Fractions . . . . . . . . . . . . . . . . . 143

$5.6 \quad$ Systematic Uncertainties . . . . . . . . . . . . . . . . . 145

5.6.1 The $B$ Meson $p_{T}$ Spectrum . . . . . . . . . . 145

$5.6 .2 \quad B$ and $D$ Lifetimes . . . . . . . . . . . . 146

5.6 .3 XFT Simulation . . . . . . . . . . . . . . . . . 147

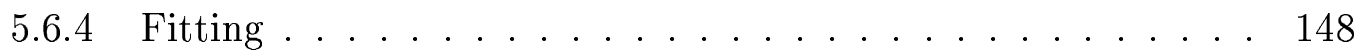

5.6 .5 Cut Efficiencies . . . . . . . . . . . . . . 152

5.6.6 The $\phi^{0}$ Invariant Mass Requirement . . . . . . . . . . . 153

5.6.7 Summary of Systematic Uncertainties . . . . . . . . . . . 154

5.7 Measurement Result . . . . . . . . . . . . . . . . 156

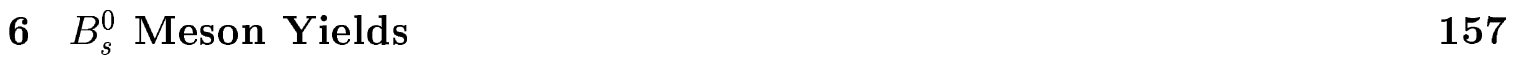

6.1 Design Yield Studies . . . . . . . . . . . . . . . . 157

6.1.1 Event Generation . . . . . . . . . . . . . . . 158

6.1.2 Level 1 Parametric Trigger Simulation . . . . . . . . . 158

6.1.3 Level 2 Parametric Trigger Simulation . . . . . . . . . . 159

6.1.4 Tracking Fiducial Volume Simulation . . . . . . . . . . . 159

6.1 .5 Yield Estimate . . . . . . . . . . . . . . . . . 159

6.2 Yield Reduction Effects . . . . . . . . . . . . . . . . 160

6.2 .1 Dead Silicon Regions . . . . . . . . . . . . . . 160

6.2 .2 Wedge Crossers . . . . . . . . . . . . . . . . . 161

6.2.3 Barrel Crossers . . . . . . . . . . . . . . . . . . . . 161

6.2 .4 Inefficiencies . . . . . . . . . . . . . . . . 162

6.2 .5 Yield Reduction . . . . . . . . . . . . . . . 168

6.3 Triggers With Loose Requirements _ . . . . . . . . . . . 170

6.4 Updated Yield Projections . . . . . . . . . . . . . . . . 170 
6.4.1 Preshutdown Inefficiencies and SVT Improvements . . . . . 172

6.4.2 SVT Algorithm Four out of Five . . . . . . . . . . . . . 172

6.4.3 Dynamically Prescaled Triggers . . . . . . . . . . . 173

6.4.4 Level 1 Rate Limitation . . . . . . . . . . . . . . 177

6.4.5 Projected Yields . . . . . . . . . . . . 177

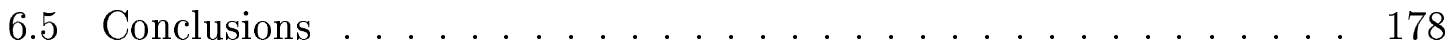

7 Conclusion

179 


\section{List of Figures}

2-1 The only first order Feynman diagram for $K^{0} \rightarrow \mu^{+} \mu^{-}$decays prior to the GIM mechanism .................. 25

2-2 Additional diagram contributing to $K^{0} \rightarrow \mu^{+} \mu^{-}$decays due to the GIM mechanism. ................... 26

2-3 The Unitarity Triangle in the complex plane. . . . . . . . . . 27

2-4 Regions of $68 \%$ and $95 \%$ probability for the fit result of the Unitarity Triangle parameters $(\rho, \eta)$, overlaid on experimental constraints . . 28

2-5 Feynman diagrams contributing to $B_{s}^{0}$ and $B^{0}$ mixing. . . . . . . 31

2-6 The tree level Feynman diagram describing $B \rightarrow D \pi^{+}$decays . . . . . 34

2-7 The additional diagram contributing only to $B^{+} \rightarrow \overline{D^{0}} \pi^{+}$decays . . . 37

3-1 Layout of the Fermilab accelerator complex . . . . . . . . . . 40

3-2 Peak luminosities for stores collided between April 2001 and September

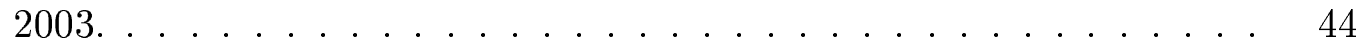

3-3 The CDF-II detector with quadrant cut to expose the different subde-

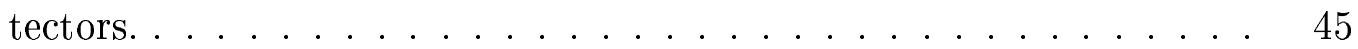

3-4 A diagram of the CDF-II tracker layout showing the different subde-

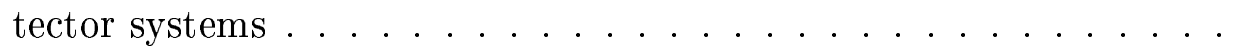

3-5 Coverage of the different silicon subdetector systems projected into the $r-z$ plane. The $r$ and $z$ axes have different scales. $\ldots . . . . . .49$

3-6 Layout of wire planes on a COT endplate . . . . . . . . . 50

3-7 Layout of wires in a COT supercell . . . . . . . . . . 51 
3-8 Dependence of the reconstructed invariant mass of $J / \psi \rightarrow \mu^{+} \mu^{-}$decays on the transverse momentum of the $J / \psi$. . . . . . . . . . 54

3-9 Rate of kaon and pion tracks faking muon signals in the CDF-II detector. 58 3-10 Diagram of the CDF-II trigger system . . . . . . . . . . 60

3-11 Diagram of the different trigger paths at Level 1 and 2. . . . . . . . 61

3-12 SVT principle of operation . . . . . . . . . . . 63

3-13 SVT impact parameter resolution . . . . . . . . . . . 63

3-14 Principle of Event Building and Level 3 Filtering . . . . . . . . . 65

4-1 Matching SVT quantities to offline quantities. . . . . . . . . 87

4-2 Mass spectrum of $B^{+}, B^{0}$ and $B_{s}^{0}$ decays using unoptimized selection

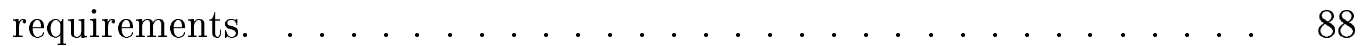

4-3 Typical topology of a $B \rightarrow D \pi$ decay. . . . . . . . . . . 89

4-4 Determining the number of background and signal events. . . . . . 89

4-5 An example of variables monitored during the optimization procedure. $\quad 90$

4-6 Mass spectra of $B^{+}, B^{0}$ and $B_{s}^{0}$ mesons using optimized selection requirements. . . . . . . . . . . . . . . . 91

5-1 Composition of the single- $B$ background for $B^{+}$decays. . . . . . . . 99

5-2 Templates used in $B^{+} \rightarrow D^{0} \pi$ fitting. . . . . . . . . . . . . . . . 99

5-3 Composition of the single- $B$ background for $B^{0}$ decays. . . . . . . . . 100

5-4 Templates used in $B^{0} \rightarrow D^{+} \pi$ fitting. . . . . . . . . . . . . . 100

5-5 Composition of the single- $B$ background for $B_{s}^{0}$ decays. . . . . . . . 101

5-6 Templates used in $B_{s} \rightarrow D_{s} \pi$ fitting. . . . . . . . . . . . . . 101

5-7 Invariant mass distribution for $B^{+} \rightarrow D^{0} \pi^{+}$candidates reconstructed with optimized selection requirements. . . . . . . . . . . . . 110

5-8 Invariant mass distribution for $B_{s}^{0} \rightarrow D_{s} \pi^{+}$candidates reconstructed with optimized selection requirements. . . . . . . . . . . . 111

5-9 Invariant mass distribution for $B^{0} \rightarrow D^{-} \pi^{+}$candidates reconstructed with optimized selection requirements. . . . . . . . . . . . 112 
5-10 Signal and high-mass sideband region definitions for sideband subtrac-

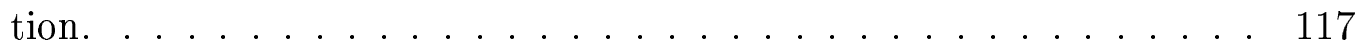

5-11 Fit of the high-mass background to an exponential distribution. . . . 118

5-12 Comparison of Data and Monte Carlo distributions of the $p_{T}(B)$ for $B^{+}$decays. . . . . . . . . . . . . . . 119

5-13 Comparison of Data and Monte Carlo distributions of the $p_{T}(B)$ for $B^{0}$ decays. . . . . . . . . . . . . . . . 119

5-14 Comparison of Data and Monte Carlo distributions of the $\phi_{0}(B)$. . 120

5-15 Comparison of Data and Monte Carlo distributions of the $\phi_{0}(B)$. . 120

5-16 Comparison of Data and Monte Carlo distributions of the $\eta(B)$ for $B^{+}$ decays. . . . . . . . . . . . . . . . 121

5-17 Comparison of Data and Monte Carlo distributions of the $\eta(B)$ for $B^{0}$ decays. . . . . . . . . . . . . . . . . 121

5-18 Comparison of Data and Monte Carlo distributions of the $p_{T}(D)$. . 122

5-19 Comparison of Data and Monte Carlo distributions of the $p_{T}(D)$. . 122

5-20 Comparison of Data and Monte Carlo distributions of the $\chi^{2}(B) . \quad \ldots 123$

5-21 Comparison of Data and Monte Carlo distributions of the $\chi^{2}(B)$. . 123

5-22 Comparison of Data and Monte Carlo distributions of the $\chi^{2}(D)$. . 124

5-23 Comparison of Data and Monte Carlo distributions of the $\chi^{2}(D)$. . 124

5-24 Comparison of Data and Monte Carlo distributions of the $L_{x y}(B)$. . 125

5-25 Comparison of Data and Monte Carlo distributions of the $L_{x y}(B)$. . 125

5-26 Comparison of Data and Monte Carlo distributions of the $d_{0}\left(\pi_{B}\right)$. 126

5-27 Comparison of Data and Monte Carlo distributions of the $d_{0}\left(\pi_{B}\right) . \quad$. 126

5-28 Comparison of Data and Monte Carlo distributions of the $p_{T}\left(\pi_{B}\right)$. . 127

5-29 Comparison of Data and Monte Carlo distributions of the $p_{T}\left(\pi_{B}\right)$. . 127

5-30 Comparison of Data and Monte Carlo distributions of the $d_{0}(B)$ for $B^{+}$decays. . . . . . . . . . . . . . . 128

5-31 Comparison of Data and Monte Carlo distributions of the $d_{0}(B)$ for $B^{0}$ decays. . . . . . . . . . . . . . . 128 
5-32 Comparison of Data and Monte Carlo distributions of the $\Delta R\left(D, \pi_{B}\right)$ for $B^{+}$decays. . . . . . . . . . . . . . . . . 129

5-33 Comparison of Data and Monte Carlo distributions of the $\Delta R\left(D, \pi_{B}\right)$ for $B^{0}$ decays. . . . . . . . . . . . . . . . 129

5-34 Comparison of Data and Monte Carlo distributions of the $L_{x y}(D)$ with respect to the primary vertex. . . . . . . . . . . 130

5-35 Comparison of Data and Monte Carlo distributions of the $L_{x y}(D)$ with respect to the primary vertex. . . . . . . . . . . 130

5-36 Comparison of Data and Monte Carlo distributions of the $L_{x y}(D) \ldots 131$

5-37 Comparison of Data and Monte Carlo distributions of the $L_{x y}(D) \ldots 131$

5-38 Comparison of Data and Monte Carlo distributions of the $\Delta \phi$ between the two trigger tracks. . . . . . . . . . . . . . . . 132

5-39 Comparison of Data and Monte Carlo distributions of the $\Delta \phi$ between the two trigger tracks. . . . . . . . . . . . . . . . . 132

5-40 Comparison of Data and Monte Carlo distributions of the $\Delta \eta$ between the two trigger tracks. . . . . . . . . . . . . . . . 133

5-41 Comparison of Data and Monte Carlo distributions of the $\Delta \eta$ between the two trigger tracks. . . . . . . . . . . . . .

5-42 Comparison of Data and Monte Carlo distributions of the $\sum p_{T}$ of the two trigger tracks. . . . . . . . . . . . . . . . . . 134

5-43 Comparison of Data and Monte Carlo distributions of the $\sum p_{T}$ of the two trigger tracks. . . . . . . . . . . . . . . . . . . 134

5-44 Fits of the $p_{T}$ spectra of the $B$ mesons for sideband subtracted data (solid) and Monte Carlo (dashed) . . . . . . . . . . . . 136

5-45 Monte Carlo reweighting functions are ratios of the fits of the individual spectra . . . . . . . . . . . . . . . . 136

5-46 Variation of trigger efficiencies as a function of run conditions. . . . . 141

5-47 Variation of reconstruction efficiencies as a function of run conditions. 141

5-48 Variation of relative total efficiencies for different run conditions. . . . 142 
5-49 Fit results for extended fit range, as part of estimating the systematic uncertainty due to limitations of the fitting model. . . . . . . . . . 149

5-50 Comparison of $\phi^{0}$ mass cut efficiency in Monte Carlo and data. . . . . 155

6-1 Track hit occupancy in wedges and halfbarrels of the SVX. . . . . . 165

6-2 Tracks lost due to wedge boundary crossing. . . . . . . . . . 166

6-3 Tracks lost due to barrel and half-barrel boundary crossing. . . . . . . 167

6-4 A sketch of the phase space overlaps of the three main hadronic $B$ paths.175

6-5 Profile of instantaneous luminosities in the Month of May 2003. . . . 175

6-6 The effective $B_{s}$ cross section as a function of the Level 1 rate limitation.176

6-7 The inverse of the prescale factor in dependence of the instantaneous luminosity. . . . . . . . . . . . . . . . . . . . 176 


\section{List of Tables}

3.1 Accelerator parameters for Run I and Run II configurations . . . . . 43

3.2 Relevant parameters for the layout of the sensors of different SVX-II

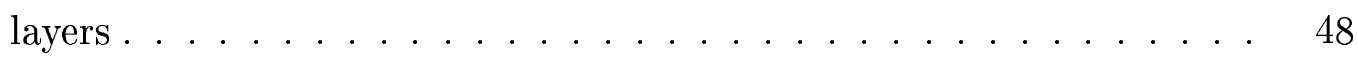

3.3 Pseudorapidity coverage, energy resolution and thickness for the different calorimeter subdetectors. . . . . . . . . . . . 57

4.1 Luminosities corresponding to the different versions of the B_CHARM trigger path. .......................... 72

4.2 Run ranges and luminosities covered by different versions of the B_CHARM_LOWPT

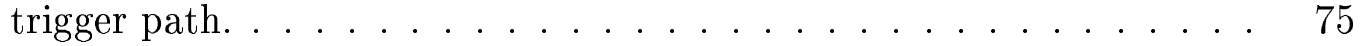

4.3 Run ranges and luminosities covered by different versions of the B_CHARM_HIGHPT

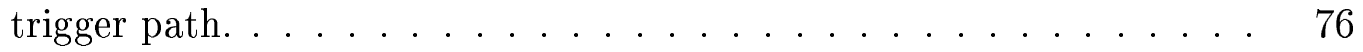

4.4 Nominal trigger requirements for the B_CHARM, B_CHARM_LOWPT and B_CHARM_HIGHPT trigger paths at Level 1 and Level 2. . . . . . . 78

4.5 Run ranges and luminosities for the seven track triggers of the B_CHARM and B_CHARM_HIGHPT trigger path families. . . . . . . . . 78

4.6 Optimized selection requirements for reconstructed hadronic $B$ decays $\quad 90$

5.1 Branching fractions for various $D^{*}$ decays $\ldots \ldots \ldots$. . . . . 98

5.2 Shape-defining template parameters and errors extracted from $D^{0} X$ generic Monte Carlo. . . . . . . . . . . . . . . 108

5.3 Shape-defining template parameters and errors extracted from $D_{s} X$ generic Monte Carlo. . . . . . . . . . . . . . 108 
5.4 Shape-defining template parameters and errors extracted from $D^{+} X$ generic Monte Carlo. . . . . . . . . . . . . . . . 109

5.5 Fit results for $B^{+} \rightarrow \overline{D^{0}} \pi^{+}$decays in the different datasets. Only statistical errors are quoted. . . . . . . . . . . . . . 113

5.6 Fit results for $B_{s}^{0} \rightarrow D_{s}^{-} \pi^{+}$decays in the different datasets. Only statistical errors are quoted. . . . . . . . . . . . . . 113

5.7 Fit results for $B^{0} \rightarrow D^{-} \pi^{+}$decays in the different datasets. Only statistical errors are quoted. . . . . . . . . . . . . . 113

5.8 Run ranges, representative runs and luminosity per run range for the hbot0h and hbot1i datasets. ................ 116

5.9 Trigger efficiencies from realistic Monte Carlo for the different $B$ decay channels and the different representative runs. . . . . . . . . . 139

5.10 Reconstruction efficiencies from realistic Monte Carlo for the different $B$ decay channels and the different representative runs. . . . . . . . 139

5.11 Luminosity averaged efficiencies from realistic Monte Carlo for the different $B$ decay channels. . . . . . . . . . . . . . . . . . 140

$5.12 B$ and $D$ meson lifetimes and errors used to estimate systematic effects by reweighting the Monte Carlo . . . . . . . . . . . . . 146

5.13 Table of systematic uncertainties due to uncertainties on meson lifetimes 147

5.14 Table of systematic uncertainties on the number of $B^{+}$events as returned by the templated fitter. . . . . . . . . . . . 150

5.15 Table of systematic uncertainties on the number of $B_{s}^{0}$ events as returned by the templated fitter. . . . . . . . . . . . 150

5.16 Table of systematic uncertainties on the number of $B^{0}$ events as returned by the templated fitter. . . . . . . . . . . . 151

5.17 Comparison of the ratio of relative $(\mathrm{N}-1)$ type efficiencies $(R)$ as measured in data and Monte Carlo for different cut variables . . . . . . . 153

5.18 Table of systematic uncertainties for the $B_{s}^{0} / B^{0}$ relative branching fraction measurements. . . . . . . . . . . . . . 155 
6.1 Gaps in the silicon coverage due to the mechanical support and readout chips. . . . . . . . . . . . . . . . . . . 163

6.2 Selection requirements applied for the two Level 2 trigger options . . 163

6.3 Measured and estimated values used as input for the $B_{s}^{0} \rightarrow D_{s}^{-} \pi^{+}$yield estimate. . . . . . . . . . . . . . . . . 163

6.4 Efficiencies and yield estimate for $B_{s}^{0} \rightarrow D_{s}^{-} \pi^{+}$decays in $2 \mathrm{fb}^{-1}$ of data.164

6.5 Comparison of design trigger-level efficiencies to those measured in unbiased data samples. . . . . . . . . . . . . . . . . . 164

6.6 Reduction of the trigger efficiency as new effects are included in the parametric simulation. . . . . . . . . . . . . . . . 164

6.7 Breakdown of event loss due to different effects which were not taken into account in the initial yield estimate. . . . . . . . . . 169 


\section{Chapter 1}

\section{Introduction}

The research status of the $B_{s}^{0}$ meson is similar to that of the recently discovered top quark in certain aspects. Both are currently only produced in $p \bar{p}$ collisions at the Tevatron. Both can provide important constraints on Standard Model parameters. In the case of the top quark, this comes from precision measurements of the top quark mass, and for the $B_{s}^{0}$ mesons, from the measurement of the frequency of $B_{s}^{0}$ oscillations.

Only a few $B_{s}^{0} \rightarrow D_{s}^{-} \pi^{+}$decay candidates have been reconstructed so far. These decays provide excellent measurements of the $B_{s}^{0}$ meson proper decay time, which is crucial for determining the frequency of $B_{s}^{0}$ oscillations. The CDF detector upgrade includes a novel displaced track trigger designed specifically for such decays. The first step towards a $B_{s}^{0}$ mixing measurement is establishing the $B_{s}^{0} \rightarrow D_{s}^{-} \pi^{+}$signal in the data gathered with the new detector and trigger system, which is the focus of this thesis. Using optimized selection requirements, the world's largest sample of fully reconstructed $B_{s}^{0} \rightarrow D_{s}^{-} \pi^{+}$decays is produced, and used to infer the ratio of branching fractions of $B_{s}^{0} \rightarrow D_{s}^{-} \pi^{+}$decays relative to $B^{0} \rightarrow D^{-} \pi^{+}$decays. Our results are relevant for planning of future measurements where higher $B_{s}^{0}$ yields can be obtained by upgrading trigger algorithms, expanding trigger paths and improvements in beam centering within the CDF-II detector.

In Chapter 2, the theoretical overview is given placing our work within the CKM formalism and $B_{s}^{0}$ mixing in particular. Chapter 3 describes the global features of the 
upgraded CDF-II detector. Event selection details and the optimization of selection requirements are described in Chapter 4. In Chapter 5, the Monte Carlo simulations are described and validated, relative efficiencies are obtained and the measurement results are summarized. The influence of future improvements needed to obtain higher $B_{s}^{0}$ yields is discussed in Chapter 6. Chapter 7 summarizes the thesis results. 


\section{Chapter 2}

\section{Theoretical Overview}

In the framework of the Standard Model, the properties of flavor-changing decays are described through the Cabibbo - Kobayashi - Maskawa (CKM) matrix. Extraction of CKM matrix elements from experimental measurements is not always trivial. Currently, there are four measurements which produce reasonably clean input into the CKM matrix: the amount of $C P$ violation in the kaon and $B$ meson system, the relative rate of $B \rightarrow \pi l \nu$ to $B \rightarrow D l \nu$ decays, and the frequency of $B^{0}$ mixing. The extrapolation of the measurement of the $B^{0}$ mixing frequency into constraints on CKM matrix elements has large errors from theoretical uncertainties on non-perturbative QCD calculations. Many of these uncertainties are common to both $B_{s}^{0}$ and $B^{0}$ mesons and cancel in the ratio of mixing frequencies. A constraint placed on the on the ratio of frequencies of $B_{s}^{0}$ and $B^{0}$ oscillations has less theoretical uncertainty. Thus, a measurement of the $B_{s}^{0}$ oscillation frequency provides a stronger constraint on the CKM matrix. With tight constraints, we can attempt to overconstrain the CKM matrix, testing the weak sector of the Standard Model.

The $B_{s}^{0}$ meson is too massive to be produced in decays of the $\Upsilon(4 S)$ resonance. As a result, $B_{s}^{0}$ mesons are not produced at any other currently operating collider. Of the two detectors currently operating at the Tevatron, the CDF-II detector has implemented a displaced track trigger which enables it to trigger on fully hadronic final states like $B_{s}^{0} \rightarrow D_{s}^{-} \pi^{+}$. Fully hadronic final states have better sensitivity to fast oscillations in mixing measurements. This puts CDF-II in the unique position 
that it has a chance of measuring the $B_{s}^{0}$ meson oscillation frequency.

This thesis presents a measurement of the branching fraction $\operatorname{Br}\left(B_{s}^{0} \rightarrow D_{s}^{-} \pi^{+}\right)$, where $D_{s}^{-} \rightarrow \phi^{0} \pi^{-}$and $\phi^{0} \rightarrow K^{+} K^{-}$. The available sample of $B_{s}^{0} \rightarrow D_{s}^{-} \pi^{+}$decays allows a precise measurement of the branching fraction and reliable estimate of the expected $B_{s}^{0}$ meson yield, which is necessary for understanding the sensitivity of the CDF-II detector to $B_{s}^{0}$ mixing.

In the following chapter, we will review the development of the CKM matrix, fitting the CKM parameters using input from experiments, the reasons for using $B_{s}^{0} \rightarrow D_{s}^{-} \pi$ decays to measure $B_{s}^{0}$ mixing and finally theoretical predictions on the value of the branching fraction $B_{s}^{0} \rightarrow D_{s}^{-} \pi$.

\subsection{The CKM Matrix}

Flavor changing decays in the Standard Model are described through weak currents. Certain symmetries of interactions through weak currents were observed early on. For instance, a lepton will only exchange a $W$ boson with the other member of its generation. For example, the $e^{-} \rightarrow W \nu_{e}$ transition will occur but the $e^{-} \rightarrow W^{-} \nu_{\mu}$ will not. This leads to the existence of the conservation of electron, muon, and tau lepton number. If quarks also only interacted within their generations, there would be similar conservation laws applying to them. Such a conservation law, however, is observed to be violated, for example in the strangeness-changing decays of the kaon, for example $K^{+} \rightarrow \mu^{+} \nu_{\mu}$.

Even before the proposal and discovery of quarks, weak hadronic currents were split into two pieces, a $\Delta S=0$ piece governing decays like $n \rightarrow p e \bar{\nu}_{e}$ and a $\Delta S=1$ piece describing decays such as $K^{+} \rightarrow m u^{+} \nu_{\mu}$. Cabibbo [1] proposed a rotation between the $\Delta S=0$ current and the $\Delta S=1$ current, by and angle $\theta_{c}$. According to Cabibbo (rephrased by Gell-Mann in terms of the quark model), the u quark coupled not to the $\mathrm{d}$, but rather to the superposition $d \cos \theta_{c}+u \sin \theta_{c}$. In this way, the $s \rightarrow W u$ transition would occur. The decay rates of strange hadrons could now be expressed in terms of $\sin \theta_{c}$. Unfortunately, this model did not always predict the 


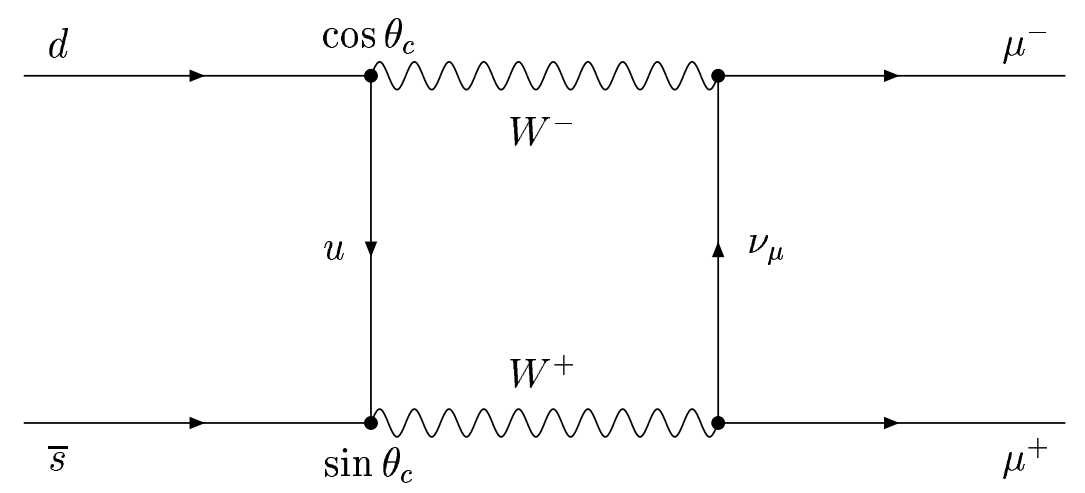

Figure 2-1: The only first order Feynman diagram for $K^{0} \rightarrow \mu^{+} \mu^{-}$decays prior to the GIM mechanism

decay rates for strange mesons very accurately. In particular, the $K^{0} \rightarrow \mu^{+} \mu^{-}$decay rate was substantially overestimated. Figure 2-1 shows the Feynman diagram that contributed to this decay in the Cabibbo model.

In 1970, Glashow, Iliopulous, and Maiani [2] predicted the existence of a fourth quark, the charm quark, in order to resolve this problem. They proposed a "mixing matrix" that would rotate the $\mathrm{d}, \mathrm{s}$ basis into the $d^{\prime}, s^{\prime}$ basis which coupled to the $\mathrm{u}$ and $\mathrm{c}$ :

$$
\left(\begin{array}{c}
d^{\prime} \\
s^{\prime}
\end{array}\right)=\left(\begin{array}{cc}
\cos \theta_{c} & \sin \theta_{c} \\
-\sin \theta_{c} & \cos \theta_{c}
\end{array}\right) \cdot\left(\begin{array}{l}
d \\
s
\end{array}\right)
$$

By doing this, they introduced a second diagram for the $K^{0} \rightarrow \mu^{+} \mu^{-}$decay, which is shown in Figure 2-2. If the charm and up quarks had the exact same mass, these two diagrams would cancel perfectly. Since their masses are not exactly the same, the new diagram suppressed the decay so that the predicted rate is consistent with experiments. This is known as the GIM mechanism.

In 1973, one year before charmonium was discovered [3, 4], Kobayashi and Maskawa [5] added a third generation of quarks (the top and bottom) to the model and generalized the GIM mixing matrix to be the most general unitary transformation from the flavor states of down-type quarks to the weak interaction states of down-type quarks. This is the CKM matrix shown in Equation 2.2. They were motivated by the fact that a violation of $C P$ symmetry was observed by Cronin and Fitch in 1964, in the decays of the $K^{0}$ meson [6]. With three generations, Kobayashi and Maskawa could incorporate $C P$ violation into the mixing matrix, while with two generations 


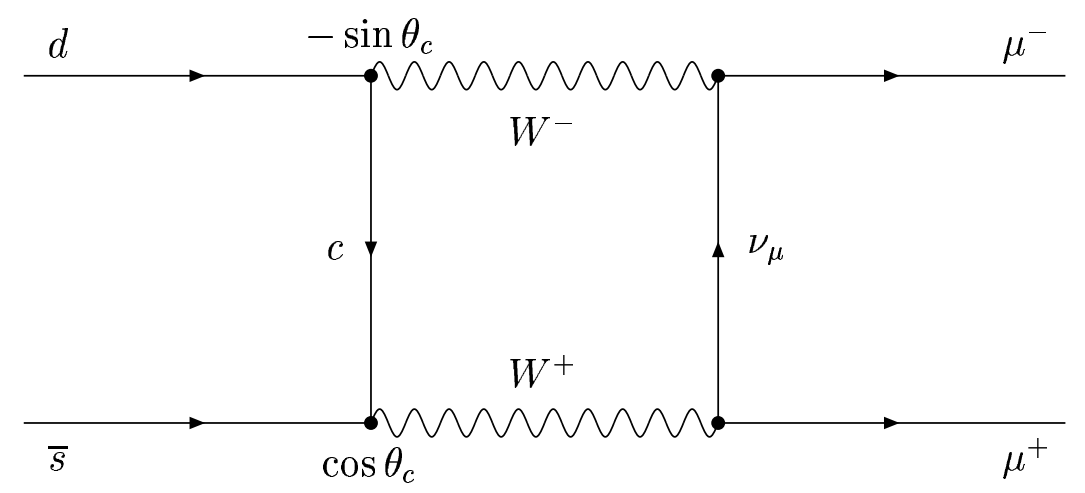

Figure 2-2: Additional diagram contributing to $K^{0} \rightarrow \mu^{+} \mu^{-}$decays due to the GIM mechanism.

they could not. Subsequently, the bottom quark was discovered in 1977 [7] and the top quark was discovered in 1993 [8], both at Fermilab.

$$
V=\left(\begin{array}{ccc}
V_{u d} & V_{u s} & V_{u b} \\
V_{c d} & V_{c s} & V_{c b} \\
V_{t d} & V_{t s} & V_{t b}
\end{array}\right)
$$

\subsubsection{Wolfenstein Parametrization and the Bjorken Triangle}

A common parametrization for the CKM matrix was given by Wolfenstein in 1983 [9]. The four parameters that define the matrix are taken to be $\lambda\left(\sin \theta_{c}\right), A, \rho$, and $\eta$, and the matrix can be written as:

$$
V=\left(\begin{array}{ccc}
1-\frac{\lambda^{2}}{2} & \lambda & A \lambda^{3}(\rho-i \eta) \\
-\lambda & 1-\frac{\lambda^{2}}{2} & A \lambda^{2} \\
A \lambda^{3}(1-\rho-i \eta) & -A \lambda^{2} & 1
\end{array}\right)
$$

This parametrization is accurate to fourth order in $\lambda$, which is roughly equal to $0.22[10]$. We can see in this parametrization that cross-generational weak decays are CKM suppressed by factors of $\lambda$. Furthermore, the on-diagonal terms of the matrix are roughly equal to 1 , and off-diagonal terms are successively smaller by factors of $\lambda$.

The unitarity condition $V V^{\dagger}=1$ yields the equations $\sum_{j} V_{i j} V_{k j}^{*}=\delta_{i k}$. The offdiagonal conditions $(i \neq k)$ can be represented as triangles in the complex plane. 


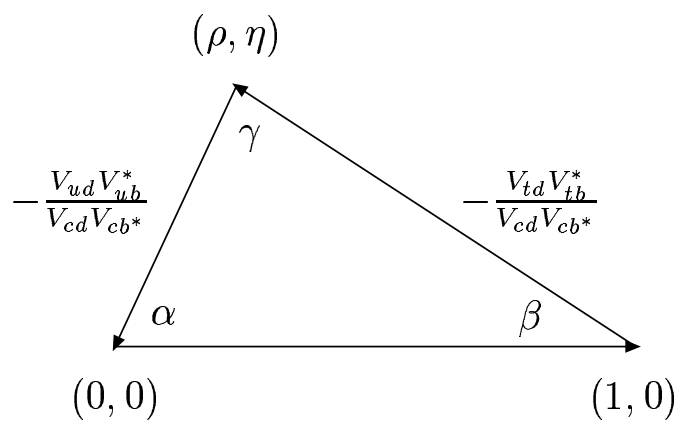

Figure 2-3: The Unitarity Triangle in the complex plane.

Each of the tree terms is a vector in the complex plane and the sum of these vectors is zero. If the CKM matrix elements had all been real numbers, this triangle would lie entirely on the real axis. The greater the $C P$ violating effects, the larger the area of the triangles.

It is easiest to measure the area of a triangle when all of the angles are roughly the same size. The triangle defined by the unitarity condition

$$
V_{u d} V_{u b}^{*}+V_{c d} V_{c b}^{*}+V_{t d} V_{t b}^{*}=0
$$

which can be written to leading order in $\lambda$ as

$$
A \lambda^{3}(\rho+i \eta)-A \lambda^{3}+A \lambda^{3}(1-\rho-i \eta)=0
$$

is roughly equilateral, with each side proportional to $\lambda^{3}$. The triangle condition expressed in Equation 2.4 is called the Bjorken (Unitarity) Triangle [11], and is shown in Figure 2-3.

If there had been more generations of quarks, Equation 2.4 would have more terms (one for each new generation) and the associated figure in the complex plane would be a polygon with the number of sides equal to the number of generations. If it is experimentally found that the Unitarity Triangle is not closed, this would be evidence for physics beyond the Standard Model. 


\subsection{Constraining the CKM Matrix}

Since the CKM matrix governs all flavor changing meson decays, one could argue that input from all measured flavor changing decays can be used to constrain parameters in the matrix. However, only a limited number of measurements provide results which can be directly interpreted in terms of constraints on the Unitarity Triangle [12]. The reason for this is that in many decays, there are considerable theoretical uncertainties involved in extracting CKM matrix elements from the measured partial decay widths.

Figure 2-4 shows a projection of current constraints on the CKM matrix parameters and regions of $68 \%$ and $95 \%$ probability regions for the fit result. The following five measurements give the cleanest constraints on the values of the parameters $\rho, \eta$ of the unitarity triangle, and are used in the fit. In Figure 2-4, these are denoted as the $\left|V_{u b} / V_{c b}\right|, \epsilon_{K}, \Delta m_{d}, \Delta m_{d} / \Delta m_{s}$, and the $\sin (2 \beta)$ constraint.

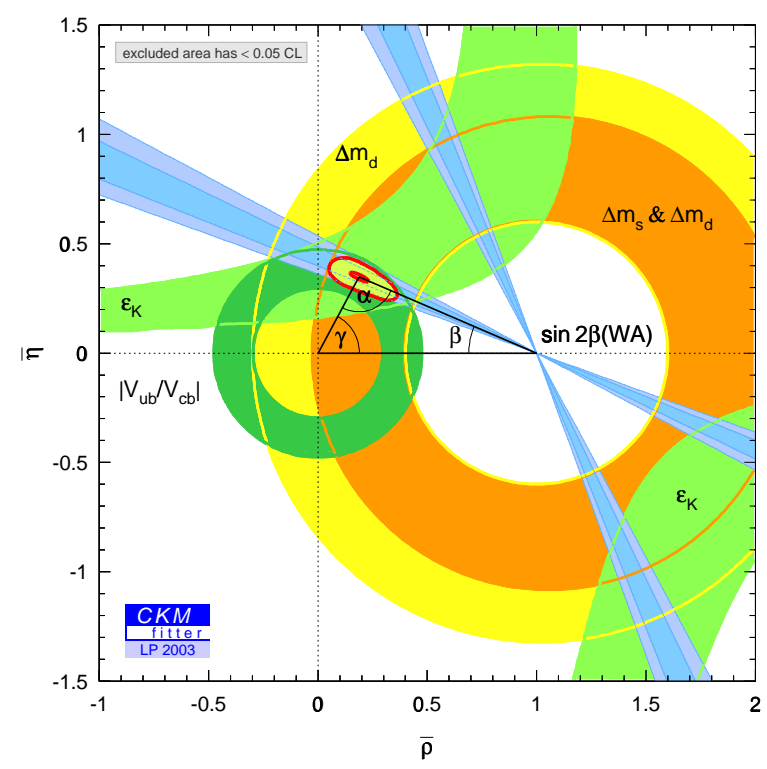

Figure 2-4: Regions of $68 \%$ and $95 \%$ probability for the fit result of the Unitarity Triangle parameters $(\rho, \eta)$, overlaid on experimental constraints

The measurement of the relative rate of $b \rightarrow c l \nu$ decays to $b \rightarrow$ ulv decays determines the $\left|V_{u b} / V_{c b}\right|$ constraint:

$$
\sqrt{\rho^{2}+\eta^{2}}=\left(1-\frac{\lambda^{2}}{2}\right) \frac{1}{\lambda}\left|\frac{V_{u b}}{V_{c b}}\right|
$$


The ratio constrains the radius of the circle centered at $(0,0)$ that passes through $(\rho, \eta)$. The best results for this measurement are obtained from the BaBar [13] and Belle [14] experiments.

The variable $\epsilon_{K}$ is defined as:

$$
\epsilon_{K}=\frac{A\left(K_{L} \rightarrow(\pi \pi)_{I=0}\right)}{A\left(K_{S} \rightarrow(\pi \pi)_{I=0}\right)}
$$

where $I$ denotes the isospin. It is a measure of $C P$ violation in the kaon system. The rates of decay are measured quite precisely in this case $\left(2.286 \pm 0.017\right.$ for $\pi^{+} \pi^{-}$and $2.275 \pm 0.017$ for $\pi^{0} \pi^{0}$ ), and the difficulty in extracting a limit on the parameters $\rho, \eta$ comes from uncertainties in the theoretical calculation, which are presently at the $15 \%$ level. A more detailed discussion on this matter can be found in Reference [12]. For our purposes, the $\epsilon_{K}$ constraint in Figure 2-4 translates roughly into a hyperbolical constraint:

$$
\eta[p \cdot(1-\rho)+q]=r
$$

in the $\eta-\rho$ plane. The exact values of parameters $p, q$ and $r$ are determined from non-perturbative QCD calculations, which introduce an error of roughly $20 \%$ (mainly from the parameters $\beta$ and $\gamma$ ) into the constraint.

Recently, the $B$ factories have measured $C P$ violation in $B^{0} \rightarrow J / \psi K_{S}^{0}$ decays $[15,16]$. The time-dependent amplitude of $B^{0} \overline{B^{0}} \rightarrow J / \psi K_{S}^{0}$ decays translates with virtually no theoretical uncertainty into a measurement of $\sin (2 \beta)$ where $\beta$ is an angle of the Unitarity Triangle, as depicted in both Figures 2-3 and 2-4. The current world average[10] value of this constraint is:

$$
(\sin 2 \beta)_{J / \psi K_{S}^{0}}=0.734 \pm 0.054
$$

and it is dominated by the measurements of the Babar and Belle collaborations.

The $\Delta m_{d}$ and $\Delta m_{d} / \Delta m_{s}$ constraints are obtained from measured frequencies of $B^{0}$ and $B_{s}^{0}$ mixing, respectively. Figure 2-5 shows the Feynman diagrams which give the main contribution to the effect of $B_{s}^{0}$ oscillations. Similar diagrams, in which the 
$t$ quarks in the loop are replaced with $c$ and $u$ quarks also contribute to the effect. To obtain the equivalent set of diagrams for $B^{0}$ oscillations, the $s$ quark lines are replaced with $d$ quark lines. Measuring only the $\Delta m_{d}$ oscillation frequency translates into a constraint on:

$$
R_{t}=\sqrt{\eta^{2}+(1-\rho)^{2}}=\frac{1}{\lambda} \cdot \frac{\left|V_{t d}\right|}{\left|V_{c b}\right|}
$$

where $R_{t}$ is the radius of a circle centered at $(1,0)$ in Figure 2-4. The propagation of the $B^{0}$ oscillation frequency into $\left|V_{t d}\right|$ involves input from lattice QCD calculations [12], which introduces uncertainties at the $20 \%$ level.

If both $\Delta m_{d}$ and $\Delta m_{s}$ are measured, then the ratio of frequencies $\Delta m_{d} / \Delta m_{s}$ translates into a constraint on:

$$
R_{t}=\frac{1}{\lambda} \xi \sqrt{\frac{m\left(B_{s}\right)}{m\left(B_{d}\right)}} \sqrt{\frac{\Delta m_{d}}{\Delta m_{s}}} \cdot\left(1-\frac{\lambda^{2}}{2}+\rho \lambda^{2}\right)
$$

where $R_{t}$ is again the radius of the same circle as before, and $\left(1-\frac{\lambda^{2}}{2}+\rho \lambda^{2}\right)$ is the departure of $\left|V_{c b}\right| /\left|V_{t s}\right|$ from unity. For $0<\rho<0.5$, this correction factor is at the $2 \%$ level and is ignored in the fit [12]. In this case, the QCD correction factors for $B_{s}^{0}$ and $B^{0}$ mesons are very similar, so the common uncertainties cancel in their ratio, $\xi$, which has a theoretical uncertainty of $\sim 3 \%$.

Due to the fact that the Unitarity Triangle is defined so that one of the sides has length 1 , three independent measurements are already enough to over-constrain the triangle. With increased samples, the BaBar and Belle experiments will improve their measurements of $\left|V_{u b} / V_{c b}\right|$ and $\sin (2 \beta)$. Proposed measurements of the angles $\alpha$ and $\gamma$ have unresolved theoretical uncertainties associated with extracting the angles from measurements, mostly from penguin diagram [17] contributions. Extrapolating constraints from the measurements of $\epsilon_{K}$ and only $\Delta m_{d}$ have large associated uncertainties from non-perturbative QCD calculations. It appears at this time that the most precise third constraint on the Unitarity Triangle will be the $\Delta m_{d} / \Delta m_{s}$ constraint. For this, it is necessary to measure the frequency of $B_{s}^{0}$ oscillations. 

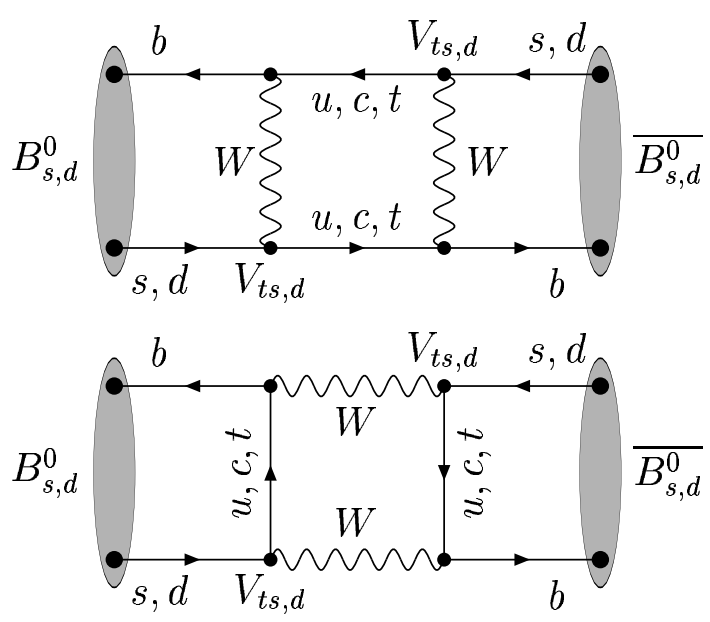

Figure 2-5: Feynman diagrams contributing to $B_{s}^{0}$ and $B^{0}$ mixing.

\subsection{Considerations for Measuring $B_{s}^{0}$ Mixing}

From an experimental point of view, the main problem in observing $B_{s}^{0}$ oscillations and measuring the $B_{s}^{0}$ oscillation frequency is the high frequency of these oscillations. The frequency of $B_{s}^{0}$ oscillations is much faster than that of $B^{0}$ oscillations. The current world average value for the frequency of $B^{0}$ oscillations is $\Delta M_{d}=0.502 \pm$ $0.006 \mathrm{ps}^{-1}$. Experimental measurements have excluded $B_{s}^{0}$ oscillations for $\Delta m_{s}>$ $14.4 \mathrm{ps}^{-1}$ at $95 \% \mathrm{CL}$.

By inspecting the Feynman diagrams in 2-5, we can also infer a rough estimate for the frequency of $B_{s}^{0}$ mixing. The main difference between $B^{0}$ and $B_{s}^{0}$ mixing are the CKM parameters $V_{t d}$ and $V_{t s}$ which govern the couplings at the corners of the box loops. The ratio of mixing probability frequencies will follow $\Delta m_{s} / \Delta m_{d} \approx\left|V_{t s} / V_{t d}\right|^{2}$, because the CKM parameters appear twice in each diagram. $\left|V_{t s} / V_{t d}\right| \sim 1 / \lambda$ which translates into $\Delta M_{s} / \Delta M_{d} \sim 25$, so the theory also predicts about 25 times faster oscillations of $B_{s}^{0}$ mesons than those of the $B^{0}$ mesons [18].

In practice, $B$ meson oscillations are measured by combining three pieces of information for every $B$ meson decay which is reconstructed: the $B$ meson flavor at production and decay, and the proper time $c t$ of the $B$ meson decay. In order to determine the decay flavor of the $B$ meson, we choose to reconstruct flavor specific final states, such as $B \rightarrow D X$. The flavor of the $D$ meson in these decays unambiguously determines the decay flavor of the $B$ meson. In order to determine the production 
flavor of the $B$ meson, we utilize flavor tagging algorithms (taggers). There are two values that characterize the usefulness of a tagging algorithm: the tagging efficiency $(\epsilon)$ and the probability that the flavor tag was correct $(p)$. The resolution with which we measure the proper decay time of the $B$ meson is $\sigma_{c t}$.

With these values, we then construct the asymmetry:

$$
\mathcal{A}(t)=\frac{N^{R S}(t)-N^{W S}(t)}{N^{R S}(t)+N^{W S}(t)}=(2 p-1) \cos (\Delta m \cdot t)
$$

where $N^{R S}(t)$ is the number of events in which the decay flavor of the $B$ meson matches the tagged production flavor of the $B$ meson, and $N^{W S}(t)$ is the number of events in which the decay flavor of the $B$ meson is opposite to the tagged production flavor of the $B$ meson. The amplitude of oscillations is called the tagging dilution $\mathcal{D}=2 p-1$. For $B$ mixing, the time dependent asymmetry will follow a cosine distribution, as shown Equation 2.12, with an amplitude which corresponds to the dilution of the tagger, $\mathcal{D}$ and a frequency which corresponds to the mixing parameter, $\Delta m$.

A Fourier analysis [19] of such an effect shows that the significance of an oscillation signal is:

$$
\mathcal{S}=\sqrt{\frac{S \epsilon \mathcal{D}^{2}}{2}} \cdot \sqrt{\frac{S}{S+B}} \cdot e^{-\frac{\sigma_{c t} \cdot \Delta m}{2}}
$$

where the first term defines the effective statistics useful for the measurement (the effective tagging probability of the tagger is $\epsilon \mathcal{D}^{2}$ ), the second term shows the deterioration of significance due to background contribution, (for zero background the second term is 1), and the third term shows the deterioration of the significance of the measurement due to uncertainty of the $B$ meson decay proper time.

We will briefly focus here on the third term, the contribution of proper decay time uncertainty. This term introduces a hard limit on the sensitivity of an experiment to fast oscillations. Roughly, we can explain this effect as follows. When the resolution of proper time measurements becomes comparable to the oscillation period, oscillations get smeared out by the measurement uncertainties and can not be resolved anymore. 
The proper time resolution can be broken down into two contributions:

$$
\sigma_{c t}=\frac{\sigma_{L x y}}{\beta \gamma} \oplus \frac{\sigma_{\beta \gamma}}{\beta \gamma} \cdot c t
$$

where $\sigma_{L x y}$ is the resolution with which we measure the displacement of the $B$ decay vertex with respect to the primary interaction vertex, $\beta \gamma$ is the boost factor, and $\sigma_{\beta \gamma}$ is the resolution of the boost factor measurement. Our choice of final state has some influence on the proper time resolution. For instance, in semi-leptonic $B_{s}$ decays, the energy lost due to the neutrino in the final state has to be corrected for using averaged momentum scale $(K)$ factors. This method introduces a $\sim 15 \%$ uncertainty of the boost factor. Equation 2.14 shows that this will mean a contribution of $15 \% \cdot c t$ per $B$ meson lifetime. In other words, for larger lifetimes, our $c t$ resolution will be drastically worse than for smaller lifetimes.

However, if we choose to do the measurement in a fully hadronic flavor specific final state, such as $B_{s}^{0} \rightarrow D_{s}^{-} \pi^{+}$, the mismeasurement of the boost factor originates from the uncertainty of momentum measurements in the tracking chamber, which is a $0.1 \%$ effect. The problem of resolution loss for higher $c t B$ decays is no longer present, which results in improved sensitivity. The problem with fully hadronic final states like $B_{s}^{0} \rightarrow D_{s}^{-} \pi^{+}$is that they require completely new techniques for triggering.

\subsection{Theory of Hadronic B Decays}

The previous sections have demonstrated the importance of the measurement of $B_{s}^{0}$ oscillations and the role of $B_{s}^{0} \rightarrow D_{s}^{-} \pi^{+}$decays in this measurement. In the following section, we briefly overview some of the theoretical methods used to calculate the branching fraction for this decay, which determines the yield of $B_{s}^{0}$ meson decays available for the measurement of $B_{s}^{0}$ oscillations.

Figure 2-6 depicts the tree-level Feynman diagram of the $B_{s}^{0} \rightarrow D_{s}^{-} \pi^{+}$decay. Diagrams of this kind are called spectator diagrams because the dominant dynamics of the decay come from the $b \rightarrow c W$ transition and the $s$ quark is a spectator in the 
process.

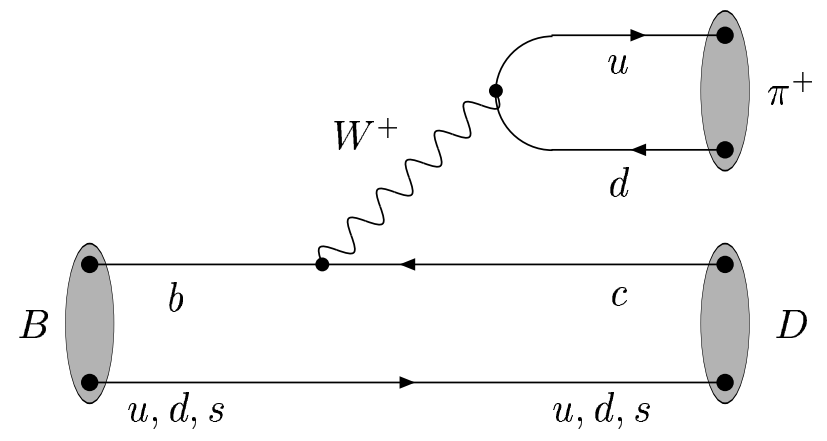

Figure 2-6: The tree level Feynman diagram describing $B \rightarrow D \pi^{+}$decays

\subsubsection{Factorization Approach}

The problem with calculating the partial width for this decay from first principles does not come from understanding the $b \rightarrow c W$ transition, but from QCD corrections which happen at the energy scale of hadronization, $\Lambda_{Q C D} \sim 100 \mathrm{MeV}$. At these energy scales, the coupling constant of $\alpha_{S}$ becomes large $(\simeq 1)$, and QCD calculations can not be expanded in this variable.

In the absence of an analytical calculation based on first principles, phenomenological approaches are often used instead. The most popular of these is the hypothesis of factorization. Immediately after the weak decay of Figure 2-6, the light $(u, \bar{d})$ quarks have a large momentum and are in the middle of a medium of gluons and light quark-antiquark pairs, with which they would interact strongly. However, if the $u \bar{d}$ pair has a small invariant mass $m(u \bar{d}) \sim m_{\pi}$, then these two quarks will remain close together as they move through the colored medium. If, in addition, they are in a color singlet state, then they will interact with the medium not individually but as a single color dipole[17]. Since the distance between the $u$ and the $\bar{d}$ grows slowly due to the small invariant mass of the two quarks, it is possible that the pair will have left the colored environment completely before its dipole moment is large enough for 
its interactions to be significant. In this case, the pair will hadronize as a single $\pi$. Such a phenomenon is known as color transparency, as the $u \bar{d}$ traveling as a coupled pair cannot see the QCD environment and passes right through it.

If, on the other hand, the $u \bar{d}$ pair has a large invariant mass, then the individual quarks will interact strongly with the medium. In this case, their reassembly into a single $\pi$ is very unlikely. As a result, it is reasonable to hypothesize the scenario of color transparency. In that case, the process factorizes into two subprocesses: the hadronization of the $D_{s}$ and, completely decoupled, the hadronization of the $\pi$. If one omits elements of the CKM matrix and propagators, for a generic $B \rightarrow D \pi$ decay, this would be equivalent to [17]:

$$
\left\langle D \pi\left|\bar{c} \gamma^{\mu}\left(1-\gamma^{5}\right) b \bar{d} \gamma_{\mu}\left(1-\gamma^{5}\right) u\right| B\right\rangle=\left\langle D\left|\bar{c} \gamma^{\mu}\left(1-\gamma^{5}\right) b\right| B\right\rangle\left\langle\pi\left|\bar{d} \gamma_{\mu}\left(1-\gamma^{5}\right) u\right| 0\right\rangle
$$

The advantage of such an approach is that the first term in the factorization, $\left\langle D\left|\bar{c} \gamma^{\mu}\left(1-\gamma^{5}\right) b\right| B\right\rangle$ is common to both semileptonic and hadronic $B$ decays so it can be inferred from semileptonic decays, and the other factor, $\left\langle\pi\left|\bar{d} \gamma_{\mu}\left(1-\gamma^{5}\right) u\right| 0\right\rangle$ is related to the pion decay form factor $f_{\pi}[20]$, which is measured in charged pion decays. The factorization ansatz makes it possible to obtain relations between various two body decays which can be tested experimentally. Unfortunately, a full calculation of the matrix element in Equation 2.15 can not be done in the factorization framework [21]. Lacking a calculation from first principles, we can attempt to infer relations between decay amplitudes for different processes using symmetries.

\subsubsection{Heavy Quark Effective Theory and Symmetries}

QCD interactions are blind to the flavor of a quark. The only parameter which differs between different flavors quarks in QCD interactions is the mass of the quark. In the case of a hadron composed of a heavy $(c, b)$ quark and a light $(u, d$ or $s)$ quark, there is a vast difference between the mass of the heavy and light quark ( 1.5 and $4.5 \mathrm{GeV} / \mathrm{c}^{2}$ compared to 1, 5 and $150 \mathrm{MeV} / \mathrm{c}^{2}$ ). Although the structure of the meson is determined by non-perturbative strong interactions, the typical energies exchanged between the 
heavy quark and the light quark and the light quark and the QCD environment are of order $\Lambda_{Q C D} \sim 100 \mathrm{MeV}<<m_{b}, m_{c}$, against which the heavy quark does not recoil. In this limit, the heavy quark acts as a static source of chromoelectric field. This is the postulate of heavy quark effective theory[22]. There are two useful symmetries that come from this theory - heavy quark symmetry and light quark symmetry. We briefly discuss heavy quark symmetry and then use light quark symmetry to establish a relation between the partial widths of $B^{0}$ and $B_{s}^{0}$ mesons.

The principle of heavy quark symmetry is a direct consequence of heavy quark effective theory. The meson is modeled with a stationary heavy quark as the source of chromoelectric field, as long as the mass of the heavy quark is much larger than the exchanged momenta ( $\sim 100 \mathrm{MeV} / \mathrm{c})$, the properties of the meson should not depend on the flavor of the heavy quark. For instance, the difference between the $D_{s}^{-}, D^{-}$and $B_{s}^{0}, B^{0}$ meson pairs is the flavor of the heavy quark. Therefore, we expect that the masses of the quarks be roughly driven by the masses of the heavy quark. The masses of the heavy quarks cancel if we compare mass differences: $m\left(D_{s}^{-}\right)-m\left(D^{-}\right)$and $m\left(B_{s}^{0}\right)-m\left(B^{0}\right)$. Measurements find the mass differences to be $99.3 \pm 0.5 \mathrm{MeV} / \mathrm{c}^{2}$ and $90.2 \pm 2.5 \mathrm{MeV} / \mathrm{c}^{2}$, respectively. The estimate obtained using heavy quark symmetry is correct to about $10 \%$.

Light quark symmetry effectively tells us that the amplitude of a diagram with a light spectator quark does not depend on the flavor of the spectator quark. The mass of the heavy quark is so large compared to the light quark masses that the differences between the light quark masses induce very small corrections to the behavior of the meson. From this we can infer that the matrix element $\left\langle D\left|\bar{c} \gamma^{\mu}\left(1-\gamma^{5}\right) b\right| B\right\rangle$ does not depend on the flavor of the light quark in the $B$ and $D$ mesons, and the amplitude of the decay driven by the diagram in 2-6 does not depend on the flavor of the light spectator quark either.

Naively, this means that the branching fractions have to be the same too, but this is not completely true. The $B^{+} \rightarrow \overline{D^{0}} \pi^{+}$decay has an additional contribution from the Feynman diagram shown in Figure 2-7 which does not apply to the decays of the $B_{s}^{0}$ and $B^{0}$ mesons. Furthermore, a branching fraction is related to the lifetime of a 


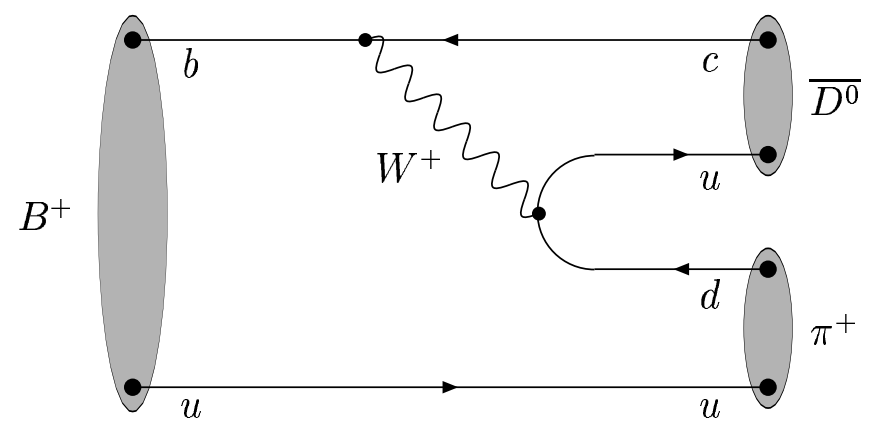

Figure 2-7: The additional diagram contributing only to $B^{+} \rightarrow \overline{D^{0}} \pi^{+}$decays

meson:

$$
B r=\frac{\Gamma_{i}}{\Gamma}
$$

where $\Gamma_{i}$ is the fractional width of the decay process and $\Gamma$ is the full width of the decay, $\Gamma=1 / \tau$. This means that the branching fractions of the mesons will be different if the lifetimes are different. The lifetimes of the $B^{+}, B^{0}$ and $B_{s}^{0}$ mesons are roughly 500, 460 and $440 \mu \mathrm{m}$ respectively. The $B_{s}^{0}$ and $B^{0}$ mesons have the same diagrams dominating their decay, and very similar lifetimes. Thus we expect the branching fractions to be the same:

$$
\operatorname{Br}\left(B_{s}^{0} \rightarrow D_{s}^{-} \pi^{+}\right) \approx B r\left(B^{0} \rightarrow D^{-} \pi^{+}\right)
$$

\subsection{Summary}

The CKM matrix is a component of the Standard Model which is related to the physics of $\mathrm{CP}$ violation and meson mixing. Much of the physics associated with the CKM matrix is represented graphically by the properties of the Unitarity triangle. By measuring the oscillation frequencies of $B_{s}^{0}$ and $B^{0}$ mesons, we constrain one of the sides of the Unitarity Triangle. Measurements from other experiments impose 
additional constraints on the Unitarity Triangle. If it is experimentally found that the Unitarity Triangle is not closed, this would be evidence for physics beyond the Standard Model. The branching fraction for the decay $B_{s}^{0} \rightarrow D_{s}^{-} \pi^{+}$determines the size of the sample available for observing $B_{s}^{0}$ oscillations at CDF-II. According to the principles of light flavor symmetry, we expect this branching fraction to be the same as the branching fraction for the decay $B^{0} \rightarrow D^{-} \pi^{+}$. 


\section{Chapter 3}

\section{Experimental Apparatus}

Fermilab's Tevatron collider represents the high energy frontier in particle physics in general. It is currently the source of the highest energy proton - antiproton $(p \bar{p})$ collisions. The collisions occur at two points in an underground ring, which has a radius of about $1 \mathrm{~km}$. At these collision points are two detectors: the Collider Detector at Fermilab (CDF-II) and D0. This analysis uses data collected by the CDF-II experiment.

Between 1997 and 2001, both the accelerator complex and the collider detectors underwent major upgrades, mainly aimed at increasing the luminosity of the accelerator, and gathering data samples of $2 \mathrm{fb}^{-1}$ or more. The upgraded machine accelerates 36 bunches of protons and anti-protons, whereas the previous version of the accelerator operated with only 6 . Consequently, the time between bunch crossings has been decreased from $3.5 \mu$ s for the previous version to $396 \mathrm{~ns}$ for the current collider.

The new configuration required detector upgrades at CDF-II to ensure a maximum response time shorter than the time between beam crossings. In the following pages, we describe how the proton and anti-proton beams are produced, accelerated to their final center of mass energy of $1.96 \mathrm{TeV}$, and collided. We then describe the components used to identify and measure properties of the particles produced in the collision. 


\subsection{Tevatron - the Source of $p \bar{p}$ Collisions}

To create the world's most powerful particle beams, Fermilab uses a series of accelerators. The diagram in Figure 3-1 shows the paths taken by protons and antiprotons from initial acceleration to collision in the Tevatron.

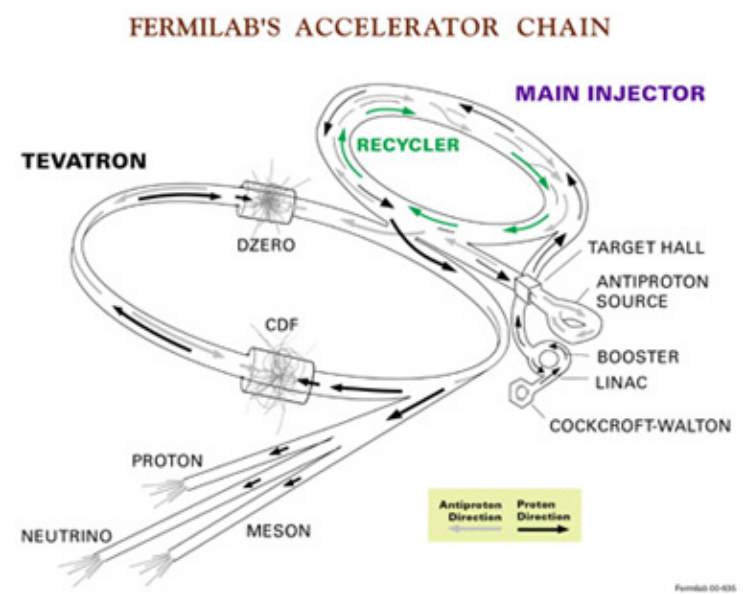

Figure 3-1: Layout of the Fermilab accelerator complex

The Cockcroft-Walton[23] pre-accelerator provides the first stage of acceleration. Inside this device, hydrogen gas is ionized to create $\mathrm{H}^{-}$ions, which are accelerated to $750 \mathrm{keV}$ of kinetic energy. Next, the $\mathrm{H}^{-}$ions enter a linear accelerator (Linac)[24], approximately 500 feet long, where they are accelerated to $400 \mathrm{MeV}$. The acceleration in the Linac is done by a series of "kicks" from RF cavities. The oscillating electric field of the RF cavities groups the ions into bunches.

The $400 \mathrm{MeV} \mathrm{H}^{-}$ions are then injected into the Booster, a circular synchrotron[24] $74.5 \mathrm{~m}$ in diameter. A carbon foil strips the electrons from the $\mathrm{H}^{-}$ions at injection, leaving bare protons. The intensity of the proton beam is increased by injecting new protons into the same orbit as the circulating ones. The protons are accelerated from $400 \mathrm{MeV}$ to $8 \mathrm{GeV}$ by a series of "kicks" applied by RF cavities. Each turn around the Booster, the protons accrue about $500 \mathrm{keV}$ of kinetic energy.

Protons are extracted from the Booster into the Main Injector [25], which operates at $53 \mathrm{MHz}$. It has four functions. It accelerates protons from $8 \mathrm{GeV}$ to $150 \mathrm{GeV}$ before injection into the Tevatron, it produces $120 \mathrm{GeV}$ protons, which are used for anti- 
proton production, it receives anti-protons from the Antiproton Source and accelerates them to $150 \mathrm{GeV}$ for injection into the Tevatron, and finally, it injects protons and antiprotons into the Tevatron.

The Main Injector replaced the Main Ring accelerator which was situated in the Tevatron tunnel. The Injector is capable of containing larger proton currents than its predecessor, which results in a higher rate of anti-proton production. The Main Injector tunnel also houses the Antiproton Recycler. Not all antiprotons in a given store are used up by the collisions. Recycling the unused antiprotons and reusing them in the next store significantly reduces the stacking time. The task of the Antiproton Recycler is to receive antiprotons from a Tevatron store, cool them and re-integrate them into the stack, so that they can be used in the next store.

To produce anti-protons, $120 \mathrm{GeV}$ protons from the Main Injector are directed into a nickel target. In the collisions, about 20 antiprotons are produced per one million protons, with a mean kinetic energy of $8 \mathrm{GeV}$. The anti-protons are focused by a lithium lens and separated from other particle species by a pulsed magnet.

Before the anti-protons can be used in the narrow beams needed in the collider, the differences in kinetic energy between the different particles need to be reduced. Since this process reduces the spread of the kinetic energy spectrum of the beam, it is referred to as "cooling" the beam. New batches of anti-protons are initially cooled in the Debuncher synchrotron, collected and further cooled using stochastic cooling [26] in the $8 \mathrm{GeV}$ Accumulator synchrotron. The principle of stochastic cooling is to sample a particles motion with a pickup sensor and correct its trajectory later with a kicker magnet. In reality, the pickup sensor samples the average motion of particles in the beam and corrects for the average. Integrated over a long period of time, this manifests itself as a damping force applied onto individual particles which evens out their kinetic energies. It takes between 10 and 20 hours to build up a "stack" of antiprotons which is then used in collisions in the Tevatron. Anti-proton availability is the most limiting factor for attaining high luminosities, assuming there are no technical problems with the accelerator (assuming, for example, perfect transfer efficiencies between accelerator subsystems) $[24,25]$. 
Roughly once a day, the stacked anti-protons (36 bunches of about $3 \times 10^{10}$ antiprotons per bunch) are injected back into the Main Injector. They are accelerated to $150 \mathrm{GeV}$ together with 36 bunches of roughly $3 \times 10^{11}$ protons. Both the protons and anti-protons are transferred to the Tevatron.

The Tevatron is the last stage of Fermilab's accelerator chain. It receives 150 $\mathrm{GeV}$ protons and anti-protons from the Main Injector and accelerates them to 980 $\mathrm{GeV}$. The protons and antiprotons circle the Tevatron in opposite directions. The beams are brought to collision at two "collision points", B0 and D0. The two collider detectors, the Collider Detector at Fermilab (CDF-II) and D0 are built around the respective collision points.

The luminosity of collisions can be expressed as:

$$
\mathcal{L}=\frac{f N_{B} N_{p} N_{\bar{p}}}{2 \pi\left(\sigma_{p}^{2}+\sigma_{\bar{p}}^{2}\right)} F\left(\frac{\sigma_{l}}{\beta^{*}}\right)
$$

where $f$ is the revolution frequency, $N_{B}$ is the number of bunches, $N_{p / \bar{p}}$ are the number of protons/anti-protons per bunch, and $\sigma_{p / \bar{p}}$ are the rms beam sizes at the interaction point. $F$ is a form factor which corrects for the bunch shape and depends on the ratio of $\sigma_{l}$, the bunch length to $\beta^{*}$, the beta function, at the interaction point. The beta function is a measure of the beam width, and is proportional to the beam's $x$ and $y$ extent in phase space.

Table 3.1 shows a comparison of Run I and design Run II[25] accelerator parameters. Figure 3-2 shows peak luminosities for stores used in this analysis.

\subsection{The CDF-II detector}

The CDF-II detector [27] is a substantial upgrade of the original CDF-II detector [28]. It is located at the B0 collision point of the Tevatron collider. The detector is designed to detect and measure properties of particles emanating from $p \bar{p}$ collisions. The design of the detector is not geared toward one particular physics measurement, but rather optimized toward extracting as a number of different properties about all 


\begin{tabular}{l|c|c} 
Parameter & Run I & Run II \\
\hline \hline number of bunches $\left(N_{B}\right)$ & 6 & 36 \\
bunch length $[\mathrm{m}]$ & 0.6 & 0.37 \\
bunch spacing $[\mathrm{ns}]$ & 3500 & 396 \\
protons/bunch $\left(N_{p}\right)$ & $2.3 \times 10^{11}$ & $2.7 \times 10^{11}$ \\
anti-protons/bunch $\left(N_{\bar{p}}\right)$ & $5.5 \times 10^{10}$ & $3.0 \times 10^{10}$ \\
total anti-protons & $3.3 \times 10^{11}$ & $1.1 \times 10^{11}$ \\
$\beta^{*}(\mathrm{~cm})$ & 35 & 35 \\
interactions/crossing & 2.5 & 2.3 \\
\hline typical luminosity $\left[\mathrm{cm}^{-2} \mathrm{~s}^{-1}\right]$ & $0.16 \times 10^{32}$ & $0.86 \times 10^{32}$ \\
integrated luminosity $\left[\mathrm{fb}^{-1}\right]$ & 3.2 & 17.3 \\
record luminosity $\left[\mathrm{fb}^{-1}\right]$ & - & $0.6 \times 10^{32}$
\end{tabular}

Table 3.1: Accelerator parameters for Run I and Run II configurations

particle species created in the $p \bar{p}$ collision. Such particle detectors are often called multi-purpose detectors.

A diagram of the CDF-II detector is shown in Figure 3-3. A quadrant of the detector is cut out to expose the different subdetectors. The detector subsystems can be grouped as follows. The innermost system is the integrated tracking system. The tracking system is barrel-shaped and consists of cylindrical subsystems which are concentric with the beam. It is designed to detect charged particles, measure their momenta and displacements from the point of collision (primary interaction vertex). The tracking system is surrounded by the Time of Flight system, designed to provide particle identification for low-momentum charged particles. Both the tracking and Time of Flight systems are placed inside a superconducting coil, which generates a $1.4 \mathrm{~T}$ solenoidal magnetic field. The coil is surrounded by calorimetry systems, which measure the energy of particles that shower when interacting with matter. The calorimetry systems are surrounded by muon detector systems. When interacting with matter, muons act as "minimally ionizing particles" - they only deposit small amounts of ionization energy in the material. Therefore, they are able to penetrate both the tracking and calorimeter systems. The integrated material of the tracking system, TOF, solenoid and calorimetry systems serves as a particle filter. Particles which penetrate through all that material are mostly muons, and they are detected 


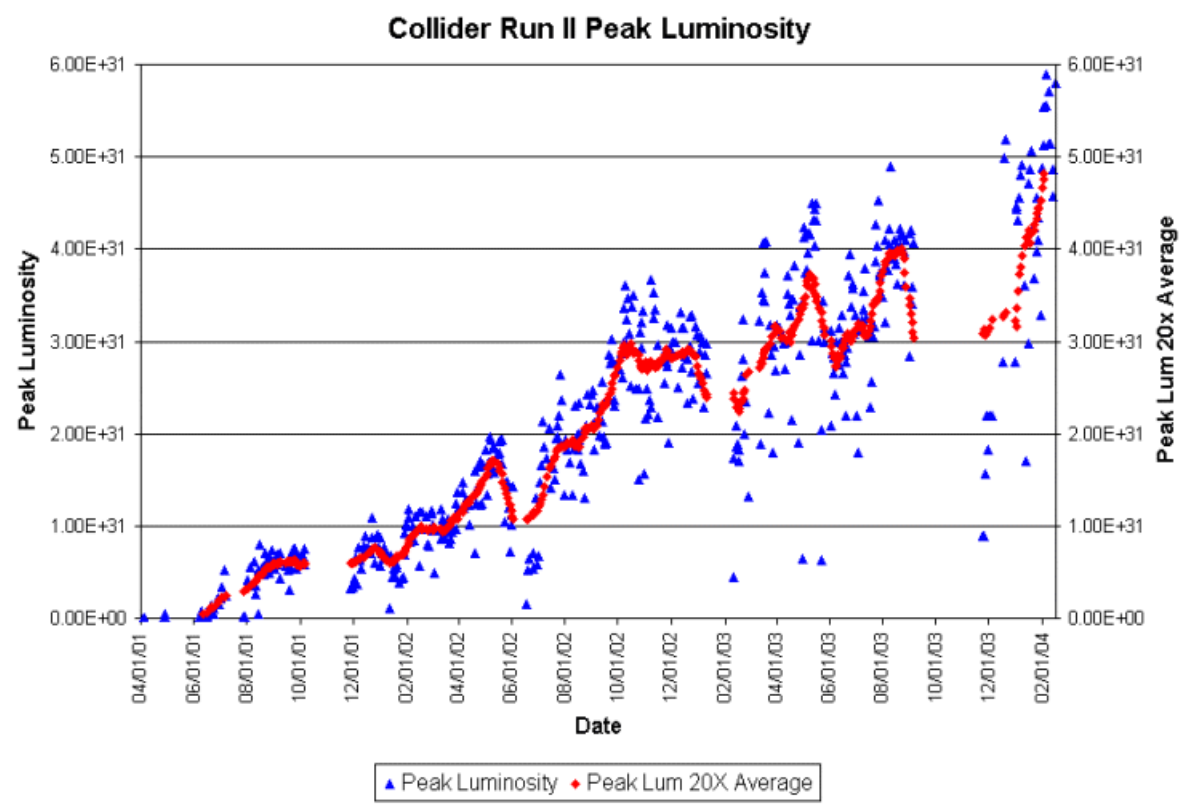

Figure 3-2: Peak luminosities for stores collided between April 2001 and September 2003.

by leaving tracks in the muon detection system, located outside of the calorimeter.

The most important parts of the detector for this analysis are the tracking system and the trigger, and these will be described in detail in the following sections. The description of the remaining systems will be brief. More detailed information on these systems can be found in the Technical Design Reports of the CDF-II [28] and CDF-II [27] detectors.

\subsection{Standard Definitions in CDF-II}

Because of the barrel-like detector shape, CDF-II uses a cylindrical coordinate system $(r, \varphi, z)$ with the origin at the center of the detector and the $z$ axis along the nominal direction of the proton beam. The $y$ axis points upwards. Since the coordinate system is right-handed, this also defines the direction of the $x$ axis. Particles moving through a homogenic solenoidal magnetic field follow helical trajectories. Reconstructed particle trajectories are referred to as "tracks". The plane perpendicular to the beam is referred to as the "transverse plane", and the transverse momentum of the track is 


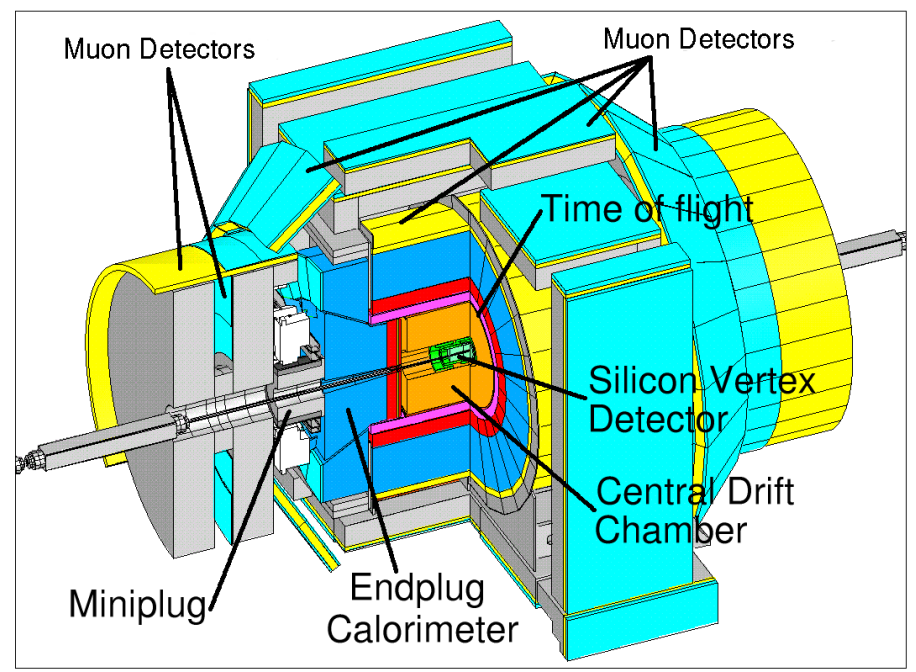

Figure 3-3: The CDF-II detector with quadrant cut to expose the different subdetectors.

referred to as $p_{T}$. As opposed to $e^{+}-e^{-}$collisions, in $p-\bar{p}$ collisions, all of the center of mass energy of the $p-\bar{p}$ system is not absorbed in the collision. The colliding partons inside the proton carry only a fraction of the kinetic energy of the proton. As a result, the center of mass system of the parton collisions is boosted along the beam direction (the "longitudinal" direction) by an unknown amount. Quantities defined in the transverse plane are conserved in the collisions. For instance, the sum of all transverse momenta of particles in the collisions is zero $\left(\sum \overrightarrow{p_{T}}=0\right)$.

To uniquely parametrize a helix in three dimensions, five parameters are needed. The CDF-II coordinate system chooses three of these parameters to describe a position, and two more to describe the momentum vector at that position. The three parameters which describe a position describe the point of closest approach of the helix to the beam line. These parameters are $d_{0}, \varphi_{0}$, and $z_{0}$, which are the $\rho, \varphi$ and $z$ cylindrical coordinates of the point of closest approach of the helix to the beam. The momentum vector is described by the track curvature $(C)$ and the angle of the momentum in the $r-z$ plane $(\cot \theta)$. From the track curvature we can calculate the transverse momentum. The curvature is signed so that the charge of the particle matches the charge of the curvature. From $\cot \theta$, we can calculate $p_{z}=p_{t} \times \cot \theta$. At any given point of the helix, the track momentum is a tangent to the helix. This 
basically means that the angle $\varphi_{0}$ implicitly defines the direction of the transverse momentum vector at the point of closest approach $p_{T}$.

The impact parameter $\left(d_{0}\right)$ of a track is another signed variable; its absolute value corresponds to the distance of closest approach of the track to the beamline. The sign of $d_{0}$ is taken to be that of $\hat{p} \times \hat{d} \cdot \hat{z}$, where $\hat{p}, \hat{d}$ and $\hat{z}$ are unit vectors in the direction of $p_{T}, d_{0}$ and $z$, respectively. An alternate variable that describes the angle between the $z$ axis and the momentum of the particle is pseudorapidity $(\eta)$ which is defined as:

$$
\eta \equiv-\ln \tan (\theta / 2)
$$

For decaying particles, we often define the displacement $L_{x y}$,

$$
L_{x y}=\vec{d} \cdot \hat{p_{T}}
$$

where $\vec{d}$ is the displacement of the decay vertex in the transverse plane, and $\hat{p_{T}}$ is the unit vector in the direction of $\overrightarrow{p_{T}}$.

\subsection{Tracking Systems}

The detector has a cylindrical tracking system immersed in a 1.4-T solenoidal magnetic field for the measurement of charged-particle momenta. We will describe this system starting from the devices closest to the beam and moving outwards. The innermost tracking device is a silicon strip vertex detector, which consists of three subdetectors. A layer of silicon sensors, called Layer 00 (L00) [29], is installed directly onto the beryllium vacuum beam pipe, at a radius of $1.7 \mathrm{~cm}$ from the beam. The beam pipe is made of beryllium because this metal has the best mechanical qualities, yet lowest nuclear interaction cross section of all materials.

The layer of silicon on the beam pipe is followed by five concentric layers of silicon sensors (SVXII) [30] located at radii between 2.5 and $10.6 \mathrm{~cm}$. The Intermediate Silicon Layers (ISL) [31] are the outermost silicon subdetector systems, consisting of 
one layer at a radius of $22 \mathrm{~cm}$ in the central region and layers at radii 20 and $28 \mathrm{~cm}$ in the forward regions. Surrounding the silicon detector is the Central Outer Tracker (COT) [32], a 3.1-m-long cylindrical open-cell drift chamber covering radii from 40 to $137 \mathrm{~cm}$.

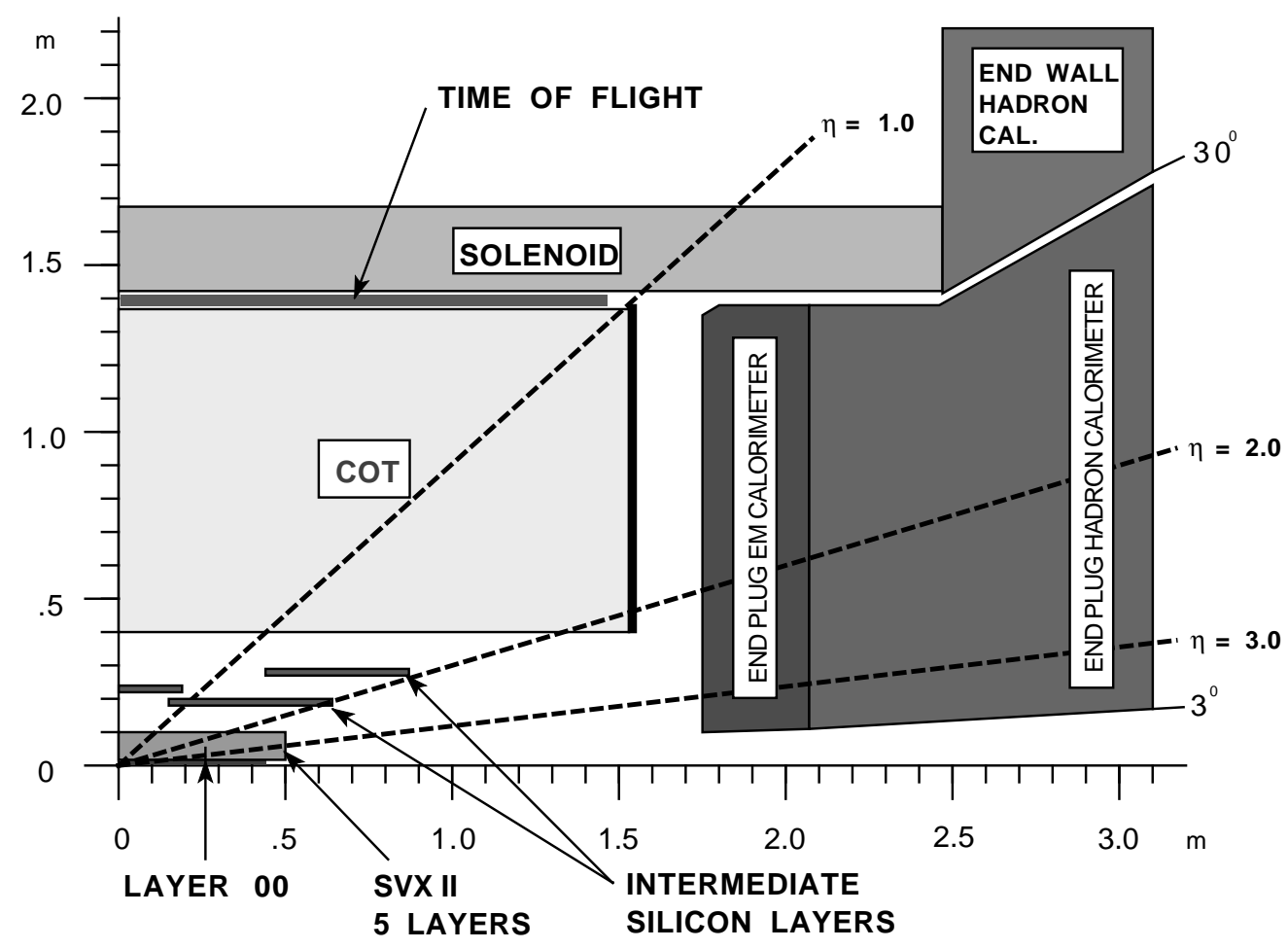

Figure 3-4: A diagram of the CDF-II tracker layout showing the different subdetector systems

\subsubsection{Silicon Tracking Detectors}

Silicon tracking detectors are used to obtain precise position measurements of the path of a charged particle. A silicon tracking detector is fundamentally a reversebiased p-n junction. When a charged particle passes through the detector material, it causes ionization. In the case of a semi-conductor material, this means that $e^{-}$-hole pairs will be produced. Electrons drift towards the anode, and holes drift toward the cathode, where the charge is gathered. The amount of charge is, to first order, 
proportional to the path length traversed in the detector material by the charged particle.

By segmenting the $\mathrm{p}$ or $\mathrm{n}$ side of the junction into "strips" and reading out the charge deposition separately on every strip, we obtain sensitivity to the position of the charged particle. All the CDF-II silicon tracking detectors are implemented as micro-strip detectors. The typical distance between two strips is about $60 \mu \mathrm{m}$. Charge deposition from a single particle passing through the silicon sensor will be read out on one or more strips. This charge deposition is called a "cluster". There are two types of microstrip detectors: single and double-sided. In single-sided detectors, only one (p) side of the junction is segmented into strips. Double-sided detectors have both sides of the junction segmented into strips. The benefit of double-sided detectors is that while one (p) side has strips parallel to the $z$ direction, providing $r-\varphi$ position measurements, the other (n) side can have strips at an angle (stereo angle) with respect to the $z$ direction, which will give $z$ position information.

The innermost layer, L00, is made of single-sided silicon sensors which provide $r-\varphi$ measurements only. The SVX-II and ISL are made of double-sided silicon sensors. As shown in Table 3.2, the SVX-II layers have different stereo angles. Two layers have a small $\left(1.2^{\circ}\right)$ stereo angle and three have a $90^{\circ}$ stereo angle. The ISL detector provides small-angle $\left(1.2^{\circ}\right)$ stereo information.

\begin{tabular}{l|c|c|c|c|c} 
Property & Layer 0 & Layer 1 & Layer 2 & Layer 3 & Layer 4 \\
\hline \hline number of $\varphi$ strips & 256 & 384 & 640 & 768 & 869 \\
number of $Z$ strips & 256 & 576 & 640 & 512 & 869 \\
stereo angle & $90^{\circ}$ & $90^{\circ}$ & $+1.2^{\circ}$ & $90^{\circ}$ & $-1.2^{\circ}$ \\
$\varphi$ strip pitch & $60 \mu \mathrm{m}$ & $62 \mu \mathrm{m}$ & $60 \mu \mathrm{m}$ & $60 \mu \mathrm{m}$ & $65 \mu \mathrm{m}$ \\
$Z$ strip pitch & $141 \mu \mathrm{m}$ & $125.5 \mu \mathrm{m}$ & $60 \mu \mathrm{m}$ & $141 \mu \mathrm{m}$ & $65 \mu \mathrm{m}$ \\
active width (mm) & 15.30 & 23.75 & 38.34 & 46.02 & 58.18 \\
active length (mm) & 72.43 & 72.43 & 72.38 & 72.43 & 72.43
\end{tabular}

Table 3.2: Relevant parameters for the layout of the sensors of different SVX-II layers

Four silicon sensors are stacked length-wise into a "ladder" structure which is $29 \mathrm{~cm}$ long. The readout electronics are mounted onto the ends of the ladders. The ladders are organized in an approximately cylindrical configuration, creating 
"barrels". A SVX-II barrel is segmented into 12 wedges, each covering approximately $30^{\circ}$ in $\varphi$ with a small overlap at the edges, allowing for many Silicon hits per track. There are three SVX-II barrels, adjacent to each other along the $z$ axis, covering the nominal interaction point in the center of the CDF-II detector. The coverage of the silicon detector subsystems is shown in Figure 3-5.

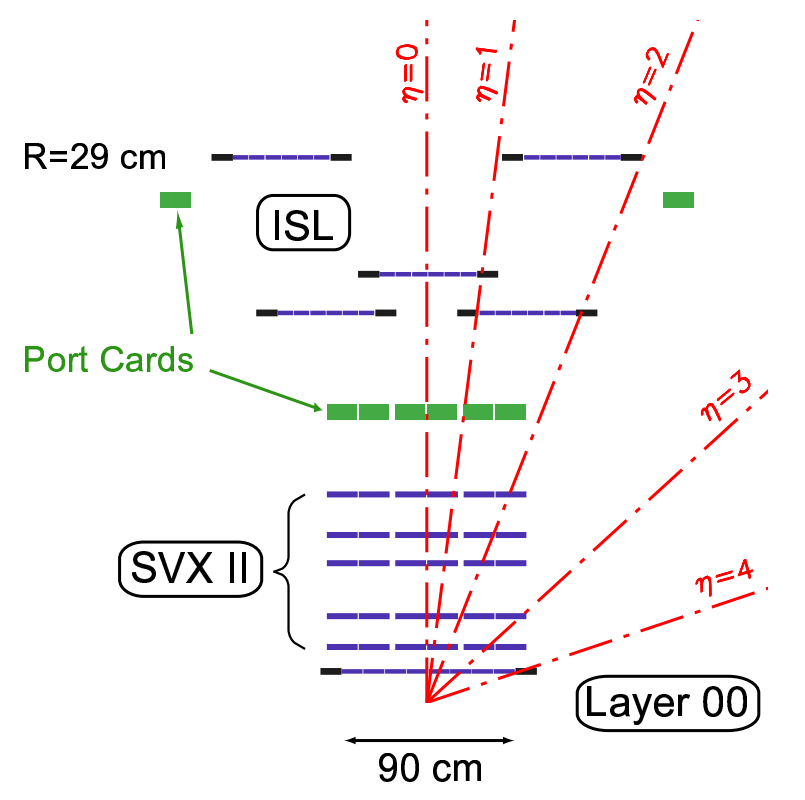

Figure 3-5: Coverage of the different silicon subdetector systems projected into the $r-z$ plane. The $r$ and $z$ axes have different scales.

\subsubsection{Central Outer Tracker}

The COT drift chamber provides accurate information in the $r-\varphi$ plane for the measurement of transverse momentum, and substantially less accurate information in the $r-z$ plane for the measurement of the $z$ component of the momentum, $p_{z}$. The COT contains 96 sense wire layers, which are radially grouped into eight "superlayers", as inferred from the end plate section shown in Figure 3-6. Each superlayer is divided in $\varphi$ into "supercells", and each supercell has 12 sense wires and a maximum drift distance that is approximately the same for all superlayers. Therefore, the number of supercells in a given superlayer scales approximately with the radius of the superlayer. The entire COT contains 30,240 sense wires. Approximately half the wires run along 
the $z$ direction ("axial"). The other half are strung at a small angle $\left(2^{\circ}\right)$ with respect to the $z$ direction ("stereo").

The active volume of the COT begins at a radius of $43 \mathrm{~cm}$ from the nominal beamline and extends out to a radius of $133 \mathrm{~cm}$. The chamber is $310 \mathrm{~cm}$ long. Particles originating from the interaction point which have $|\eta|<1$ pass through all 8 superlayers of the COT. Particles which have $|\eta|<1.3$ pass through 4 or more superlayers.

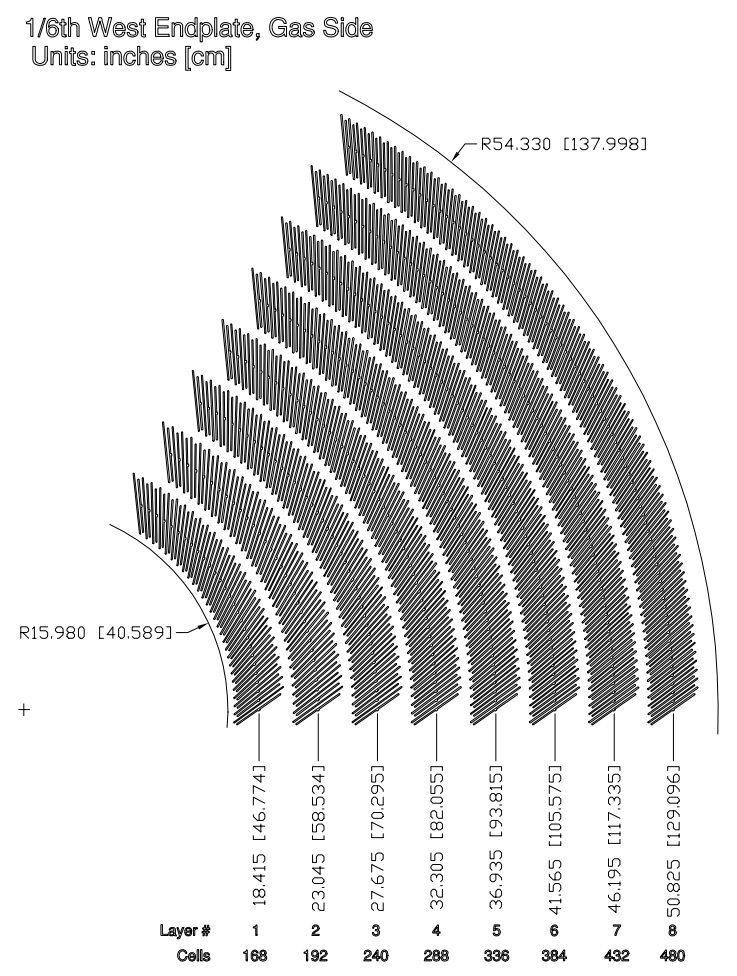

Figure 3-6: Layout of wire planes on a COT endplate

The supercell layout, shown in Figure 3-7 for superlayer 2, consists of a wire plane containing sense and potential (for field shaping) wires and a field (or cathode) sheet on either side. Both the sense and potential wires are $40 \mu \mathrm{m}$ diameter gold plated Tungsten. The field sheet is $6.35 \mu \mathrm{m}$ thick Mylar with vapor-deposited gold on both sides. Each field sheet is shared with the neighboring supercell.

The COT is filled with an Argon-Ethane-CF4 (50:35:15) gas mixture. The mixture is chosen to have a constant drift velocity across the cell width. When a charged particle passes through, the gas is ionized. Electrons drift towards the sense wires. 
The electric field in a cylindrical system grows exponentially with decreasing radius. As a result, the electric field very close to the sense wire is large, resulting in an avalanche discharge when the charge drifts close to the wire surface. This effect provides a gain of $\sim 10^{4}$. The maximum electron drift time is approximately $100 \mathrm{~ns}$. Due to the magnetic field that the COT is immersed in, electrons drift at a Lorentz angle of $\sim 35^{\circ}$. The supercell is tilted by $35^{\circ}$ with respect to the radial direction to compensate for this effect.

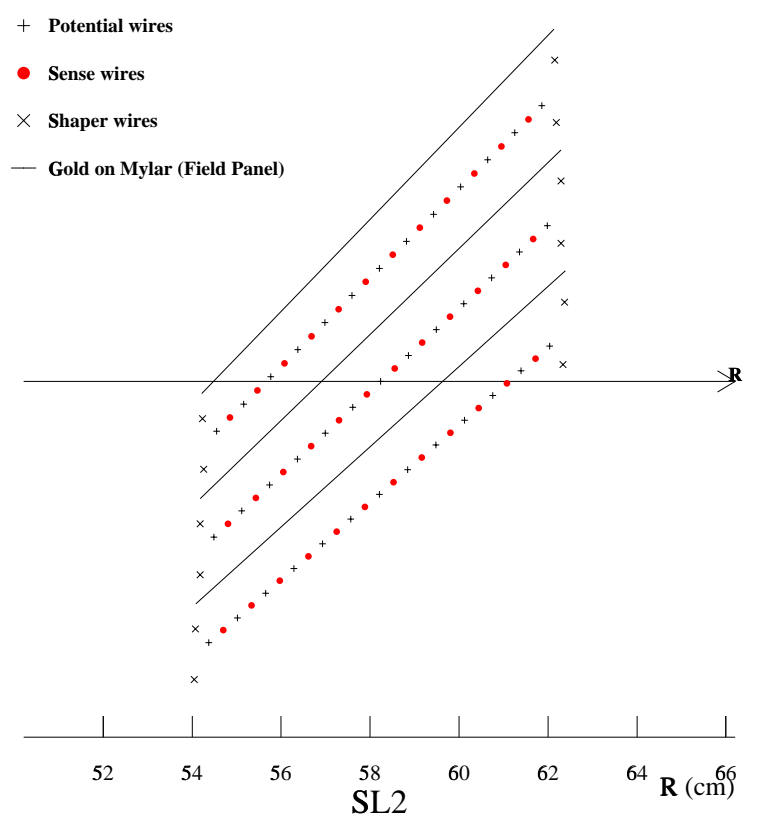

Figure 3-7: Layout of wires in a COT supercell

Signals on the sense wires are processed by the ASDQ (Amplifier, Shaper, Discriminator with charge encoding) chip, which provides input protection, amplification, pulse shaping, baseline restoration, discrimination and charge measurement [33]. The charge measurement is encoded in the width of the discriminator output pulse, and is used for particle identification by measuring the ionization along the trail of the charge particle $(d E / d x)$. The pulse is sent through $\sim 35 \mathrm{ft}$ of micro-coaxial cable, via repeater cards to Time to Digital Converter (TDC) boards in the collision hall. Hit times are later processed by pattern recognition (tracking) software to form helical tracks. 


\subsubsection{Pattern Recognition Algorithms}

As explained in the previous sections, charged particles leave small charge depositions as they pass through the tracking system. By following, or "tracking" these depositions, pattern recognition algorithms can reconstruct the charged particle track.

There are several pattern recognition algorithms used to reconstruct tracks in the CDF-II tracking system. Most of the tracks are reconstructed using "Outside-In" algorithms which we will describe here. The name of this group of algorithms suggest that the track is followed from the outside of the tracking system inward.

The track is first reconstructed using only COT information. The COT electronics report hit time and integrated charge for every wire in an event. The hit time corresponds to the time that an avalanche occurred at a sense wire. The hit time can be interpreted as the drift time of the charge in the gas, but it has to be corrected for time of flight first. The hit timing resolution is of the order of a few ns; this roughly corresponds to the average spread in collision times. It is assumed that the collision times always happen at the same time in a cycle during a store. An average of collision times is done for many previous events and this is used as the event collision time. Hit times corrected for the collision time are interpreted as drift times and used in pattern recognition. To perform the final track fit, an additional time of flight correction is performed assuming massless particles.

The helical track, when projected into the two dimensional $r-\varphi$ plane, is a circle. This simplifies pattern recognition, so the first step of pattern recognition in the COT looks for circular paths in radial superlayers of the COT. Super-cells in the radial superlayers are searched for sets of 4 or more hits that can be fit to a straight line. These sets are called "segments". The straight-line fit for a segment gives sufficient information to extrapolate rough measurements of curvature and $\varphi_{0}$. Once segments are found, there are two approaches to track finding. One approach is to link together segments for which the measurements of curvature and $\varphi_{0}$ are consistent. The other approach is to improve the curvature and $\varphi_{0}$ measurement of a segment reconstructed in superlayer 8 by constraining its circular fit to the beamline, 
and then adding hits which are consistent with this path. Once a circular path is found in the $r-\varphi$ plane, segments and hits in the stereo superlayers are added by their proximity to the circular fit. This results in a three-dimensional track fit. Typically, if one algorithm fails to reconstruct a track, the other algorithm will not. This results in a high track reconstruction efficiency $(\sim 95 \%)$ in the COT for tracks which pass through all 8 superlayers $\left(p_{T} \geq 400 \mathrm{MeV} / \mathrm{c}^{2}\right)$. The track reconstruction efficiency mostly depends on how many tracks there are to be reconstructed in the event. If there are many tracks present close to each other, hits from one track can shadow hits from the other track, resulting in efficiency loss.

Once a track is reconstructed in the COT, it is extrapolated into the SVX-II. Based on the estimated errors on the track parameters, a three-dimensional "road" is formed around the extrapolated track. Starting from the outermost layer, and working inward, silicon clusters found inside the road are added to the track. As a cluster gets added, the road gets narrowed according to the knowledge of the updated track parameters. Reducing the width of the road reduces the chance of adding a wrong hit to the track, and also reduces computation time. In the first pass of this algorithm, $r-\varphi$ clusters are added. In the second pass, clusters with stereo information are added to the track.

\subsubsection{Momentum Scale}

As the charged particle traverses through the tracker material, it loses energy. For a track that passes through the entire SVX-II volume, the amount of energy loss is roughly $9 \mathrm{MeV}$. The value is roughly constant, regardless of the momentum of the particle. In the reconstructed distribution of invariant mass of $J / \psi \rightarrow \mu^{+} \mu^{-}$decays, this effect will be more noticeable for low-momentum $J / \psi$ decays than high-momentum decays. Figure 3-8 illustrates this effect. We use the momentum-dependence of the $\mu^{+} \mu^{-}$invariant mass to calibrate the momentum scale of our detector. The $J / \psi$ mass has to be invariant of transverse momentum and match with the world average [10] value if the momentum scale is correctly calibrated. Our calibration procedure follows two steps. First, the momentum dependence of the $J / \psi$ mass is removed by correctly 
accounting for the energy loss in the tracker material, and then the overall shift of the $J / \psi$ mass is removed by correcting the value of the magnetic field used in the conversion of curvature into transverse momentum.

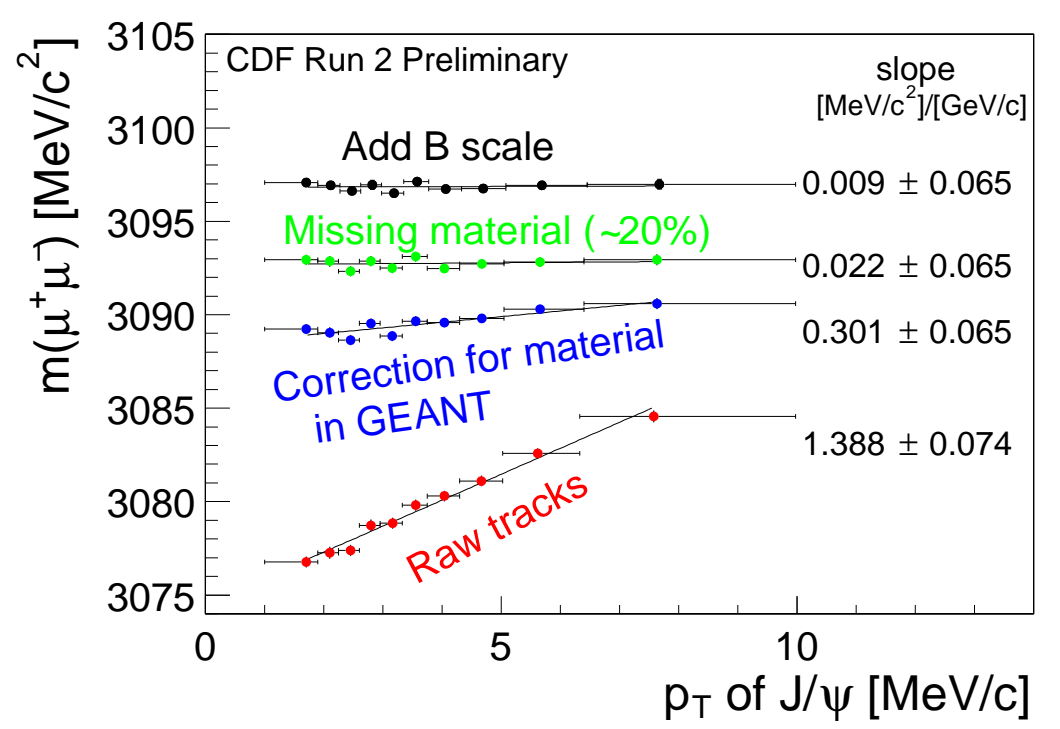

Figure 3-8: Dependence of the reconstructed invariant mass of $J / \psi \rightarrow \mu^{+} \mu^{-}$decays on the transverse momentum of the $J / \psi$.

There are two types of material in the SVX-II tracker. The silicon sensors are read out and therefore called active material. Everything else in the silicon tracker (readout chips, cards, cables, cooling pipes) is passive material. The energy loss in the active material of the tracking system is taken into account by mapping out the material in the GEANT [34] description of our detector. The passive material in the detector description is not complete, so some energy loss is unaccounted for by this method. An additional layer of material is added to the detector description, to correct for the missing material on average. By tuning the amount of missing material, the momentum dependence of the $J / \psi$ mass is removed. The remaining discrepancy with respect to the PDG average is corrected for by scaling the magnetic field. Because of the implementation of this procedure, we can not use it to measure the $J / \psi$ mass, but the results of the calibration process (the amount of missing material and the corresponding magnetic field) can be used to correct the momentum scale in any other measurement. 


\subsection{Time of Flight}

Outside the tracking system, still inside the superconducting magnetic coil, CDF-II has a Time of Flight (TOF) [35] system. The TOF system is designed to distinguish low momentum pions, kaons and protons by measuring the time it takes these particles to travel from the primary vertex of the $p \bar{p}$ collision to the TOF system. The system consists of 216 bars of scintillating material, roughly $300 \mathrm{~cm}$ in length and with a cross-section of $4 \times 4 \mathrm{~cm}$. The bars are arranged into a barrel around the COT outer cylinder. They are surrounded by superconducting solenoid on the outside. Particles passing through the scintillating material of the bars, deposit energy causing small flashes of visible light. This light is detected by photomultiplier (PMT) tubes which are attached at the end of each bar. The signal from the photomultiplier tube is processed by a pre-amplifier circuit mounted directly onto the tube. The amplified signal is sent via a twisted pair to the readout electronics in the collision hall. The readout electronics perform both time and amplitude digitization of the signal. The TDC information is a digitization of the time when the signal pulse reaches a fixed discriminator threshold. This time depends on the amplitude of the pulse, since a large pulse crosses the threshold earlier (time walk). The digitization of the pulse amplitude is needed to correct for this effect. After correcting for time walk effects, the timing resolution of the TOF system is currently about $110 \mathrm{ps}$ for particles crossing the bar exactly in front of one of the photomultiplier tubes. The timing resolution varies with displacement from the photomultiplier tube. Large pulses give better timing resolution, and light attenuates while traveling through the scintillator material. Therefore, particles passing through the bar near the photomultiplier tube have better timing resolution than those which are far away.

\subsection{Calorimeters}

The main effort of the Run II upgrade of the CDF-II calorimeter system dealt with upgrading the electronics to handle the faster bunch crossings. The active detector 
parts were taken over from Run I without modification. Since this analysis does not use calorimetry information, we will only briefly describe this system, and a more detailed description can be found in the CDF-II Technical Design Report [28].

The CDF-II calorimeter has a "projective tower" geometry. This means that it is segmented in $\eta$ and $\varphi$ "towers" that point to the interaction region. The coverage of the calorimetry system is $2 \pi$ in $\varphi$ and $|\eta|<4.2$ in pseudo-rapidity. The calorimeter system is divided into three regions: central, plug and forward. Corresponding to these regions, the subsystems will have one of the letters $\mathrm{C}, \mathrm{P}$ and $\mathrm{F}$ in their acronym. Each calorimeter tower consists of an electromagnetic shower counter followed by a hadron calorimeter. This allows for comparison of the electromagnetic and hadronic energies deposited in each tower, and therefore separation of electrons and photons from hadrons.

There are three subdetectors for the electromagnetic calorimeter: CEM, PEM and FEM. These correspond to the central, plug and forward regions of $|\eta|$, respectively. The CEM uses lead sheets interspersed with scintillator as the active detector medium. The PEM and FEM use proportional chambers. The hadron calorimeters in the central region are the central (CHA) and endwall (WHA). The plug and forward region are covered by the PHA and FHA, calorimeters, respectively. The CHA and WHA are composed of alternating layers of iron and scintillator. The PHA and FHA subdetectors are made of alternating layers of iron and gas proportional chambers. The pseudorapidity coverage, resolutions and thickness for the different electromagnetic and hadron calorimeters is given in Table 3.3.

\subsection{Muon Systems}

Muons are particles which interact with matter only by ionization. For energies relevant to this experiment, they do not cause showers in the electromagnetic or hadronic calorimeters. As a result, if a muon is created in the collision and has enough momentum, it will pass through the calorimeter with minimal interaction with the material inside. Therefore, the calorimeter can be considered as a "filter" 


\begin{tabular}{l|c|c|c} 
System & $|\eta|$ coverage & Energy Resolution & Thickness \\
\hline \hline CEM & $|\eta|<1.1$ & $13.5 \% / \sqrt{E_{T}} \oplus 3 \%$ & $18 X_{0}$ \\
PEM & $1.1<|\eta|<2.4$ & $28 \% / \sqrt{E_{T}} \oplus 2 \%$ & $18-21 X_{0}$ \\
FEM & $2.2<|\eta|<4.2$ & $25 \% / \sqrt{E_{T}} \oplus 2 \%$ & $25 X_{0}$ \\
\hline CHA & $|\eta|<0.9$ & $50 \% / \sqrt{E_{T}} \oplus 3 \%$ & $4.5 \lambda_{0}$ \\
WHA & $0.7<|\eta|<1.3$ & $75 \% / \sqrt{E_{T}} \oplus 4 \%$ & $4.5 \lambda_{0}$ \\
PHA & $1.3<|\eta|<2.4$ & $90 \% / \sqrt{E_{T}} \oplus 4 \%$ & $5.7 \lambda_{0}$ \\
FHA & $2.4<|\eta|<4.2$ & $130 \% / \sqrt{E_{T}} \oplus 4 \%$ & $7.7 \lambda_{0}$
\end{tabular}

Table 3.3: Pseudorapidity coverage, energy resolution and thickness for the different calorimeter subdetectors of the CDF-II experiment. The $\oplus$ symbol means that the constant term is added in quadrature to the resolution. $\lambda_{0}$ signifies interaction lengths and $X_{0}$ radiation lengths.

which retains particles that shower when interacting with matter and muons, and passes muons, which do not. Muon detection systems are therefore placed radially outside the calorimeters.

The CDF-II detector has four muon systems: the Central Muon Detector (CMU) [36], Central Muon Upgrade Detector (CMP) [37], Central Muon Extension Detector (CMX) [38], and the Intermediate Muon Detector (IMU). The CMU and CMP detectors are made of drift cells, and the CMX detector is made of drift cells and scintillation counters, which are used to reject background based on timing information. Using the timing information from the drift cells of the muon systems, short tracks (called "stubs") are reconstructed. Tracks reconstructed in the COT are extrapolated to the muon systems. Based on the projected track trajectory in the muon system, the estimated errors on the tracking parameters and the position of the muon stub, a $\chi^{2}$ value of the track-stub match is computed. To ensure good quality of muons, an upper limit is placed on the value of $\chi_{\varphi}^{2}$, the $\chi^{2}$ of the track-stub match in the $\varphi$ coordinate.

Most of the particles that pass through the calorimeter without showering are muons, but it is also possible for pions or kaons to survive the passage. These particles can then fake muon signals in the muon chambers. Typically, these fake rates are at the percent level, as seen in Figure 3-9 for the CMU and CMP detectors combined. The Figure shows the rate at which charged pions and kaons fake muon signals in the 
muon systems. The difference between $K^{+}$and $K^{-}$rates comes from the different cross section for interaction of these two mesons with the calorimeter material. The different interaction cross section for these two mesons comes from their quark content. In the $K^{+}$, the strange quark is the anti-quark
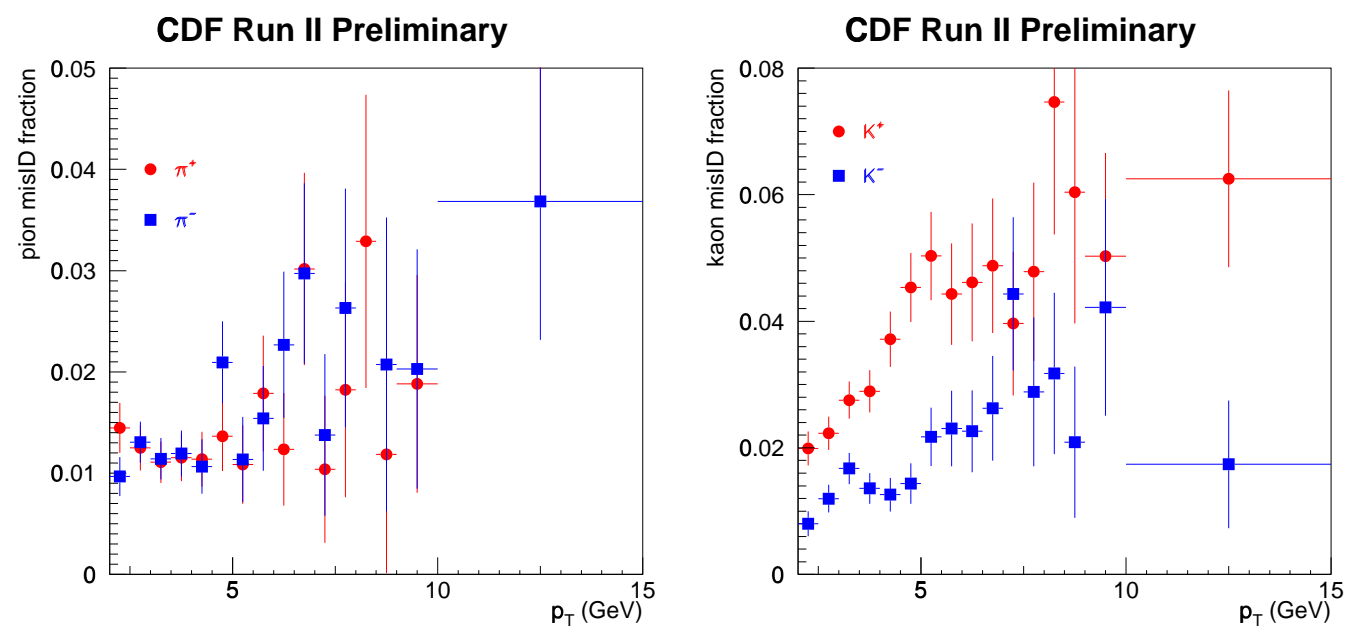

Figure 3-9: Rate of kaon and pion tracks faking muon signals in the CDF-II detector. Roughly $1 \%$ of all pions(left) and $2-4 \%$ of all kaons (right) will fake a muon signal.

\subsection{Triggering}

Triggering systems are necessary because it is not physically possible to store information about every single $p \bar{p}$ collision. Collisions happen roughly at a rate of 2.5 $\mathrm{MHz}$, and the readout of the full detector produces an event roughly the size of 250 $\mathrm{kB}$. There is no medium available which is capable of recording data this quickly, nor would it be practical to analyze all this data later on. The trigger system is a pre-filter, which reduces data rates and volumes to manageable levels.

The CDF-II triggering system is designed based on three conditions. The first condition is that the trigger be deadtimeless. This means that the trigger system has to be quick enough to make a decision for every single event, before the next event occurs. The second condition is imposed by the Tevatron upgrade for Run II, and it is the expected time between collisions, 132 ns. The last condition is that the data 
logging system can write about 30-50 events per second to tape, because of limited resources (money needed to buy computational power to do analysis on the data). In short, the trigger has to be fast enough to analyze every collision, and it has to figure out which 50 of 2.5 million events it should save in a given second. This is achieved by staging trigger decisions in three levels, as shown in Figure 3-10.

Each level of the trigger is given a certain amount of time to reach a decision about accepting or rejecting an event. By increasing the time allowed for triggering at different levels of the trigger, the complexity of reconstruction tasks can be increased at every level. At the first level of the trigger, only very rough and quick pattern recognition and filtering algorithms are used. In order to do this in time, the Level 1 and Level 2 triggering mechanisms are implemented with custom electronics. The third level of the trigger is implemented with a PC farm with about 300 CPU's. Using each CPU as an event buffer allows for nearly one second to be allocated for the trigger decision. As a result, nearly offline quality of event reconstruction is available at the third level of triggering. The Level 3 rejection rate is about 10 , resulting in 30 events/sec being accepted by the Level 3 trigger and written to tape.

The delay necessary to make a trigger decision is achieved by storing detector readout information in a storage pipeline. At Level 1, for every Tevatron clock cycle, the event is moved up one slot in the pipeline. By the time it reaches the end of the pipeline, the trigger will have reached a decision whether to accept or reject this event. If the event is accepted, its information will be sent to the higher level of the trigger. Otherwise, the event is simply ignored. Since the Level 1 buffer has 42 slots, the time allocated for making a trigger decision is about $5 \mu s$. The rejection factor after Level 1 is about 150, so the Level 1 accept rate is below $50 \mathrm{kHz}$. At Level 2, there are 4 event buffers available. This allows for $20 \mu$ s for the trigger decision. The Level 2 rejection factor is again around 150, and the accept rate is around $300 \mathrm{~Hz}$.

A set of requirements that an event has to fulfill at Level 1, Level 2 and Level 3 constitutes a trigger path. Requiring that an event was accepted through a well defined trigger path eliminates volunteer events. A volunteer event is an event which passed a higher level (L2, L3) trigger requirement but did not pass the preceding lower 


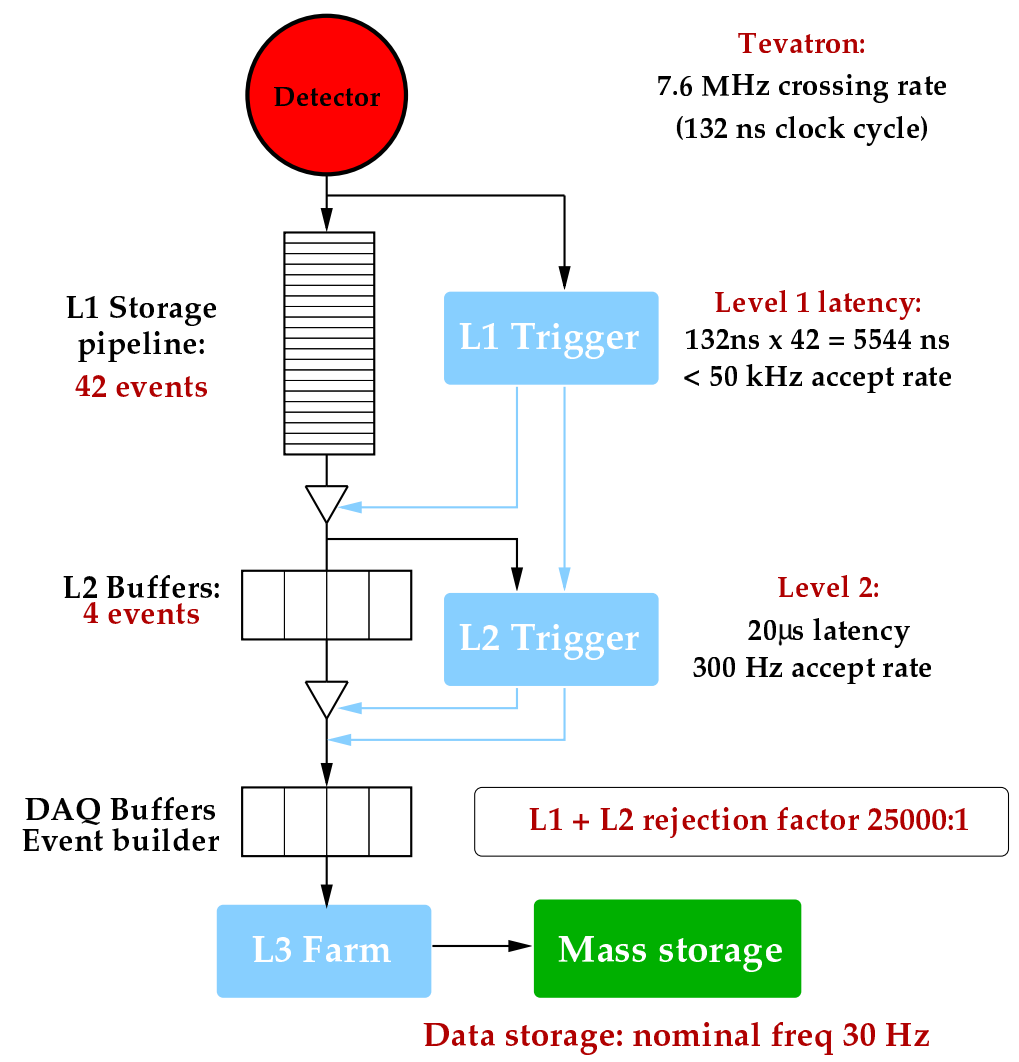

Figure 3-10: Diagram of the CDF-II trigger system

level (L1, L1/L2) trigger requirement. The CDF-II trigger system implements about 100 degenerate trigger paths. An event will be accepted if it passes the requirements of any one of these paths. The trigger path used in this analysis is the "Two Track" trigger path, which is shown in Figure 3-11, and which we will describe in detail here. The trigger path

is optimized for finding charm and bottom mesons that decay in hadronic final states. The strategy of the trigger path is as follows. At Level 1, rough measurements of track momenta are available. By cutting on track momenta and angles, most of the inelastic background will be rejected. At Level 2, the additional time available for reconstruction is utilized to use SVX-II information and obtain better impact parameter measurements of the tracks. Requiring non-zero impact parameters of tracks will require that they come from decays of long-lived particles - charmed and bottom mesons. 


\section{CDF Detector Components}

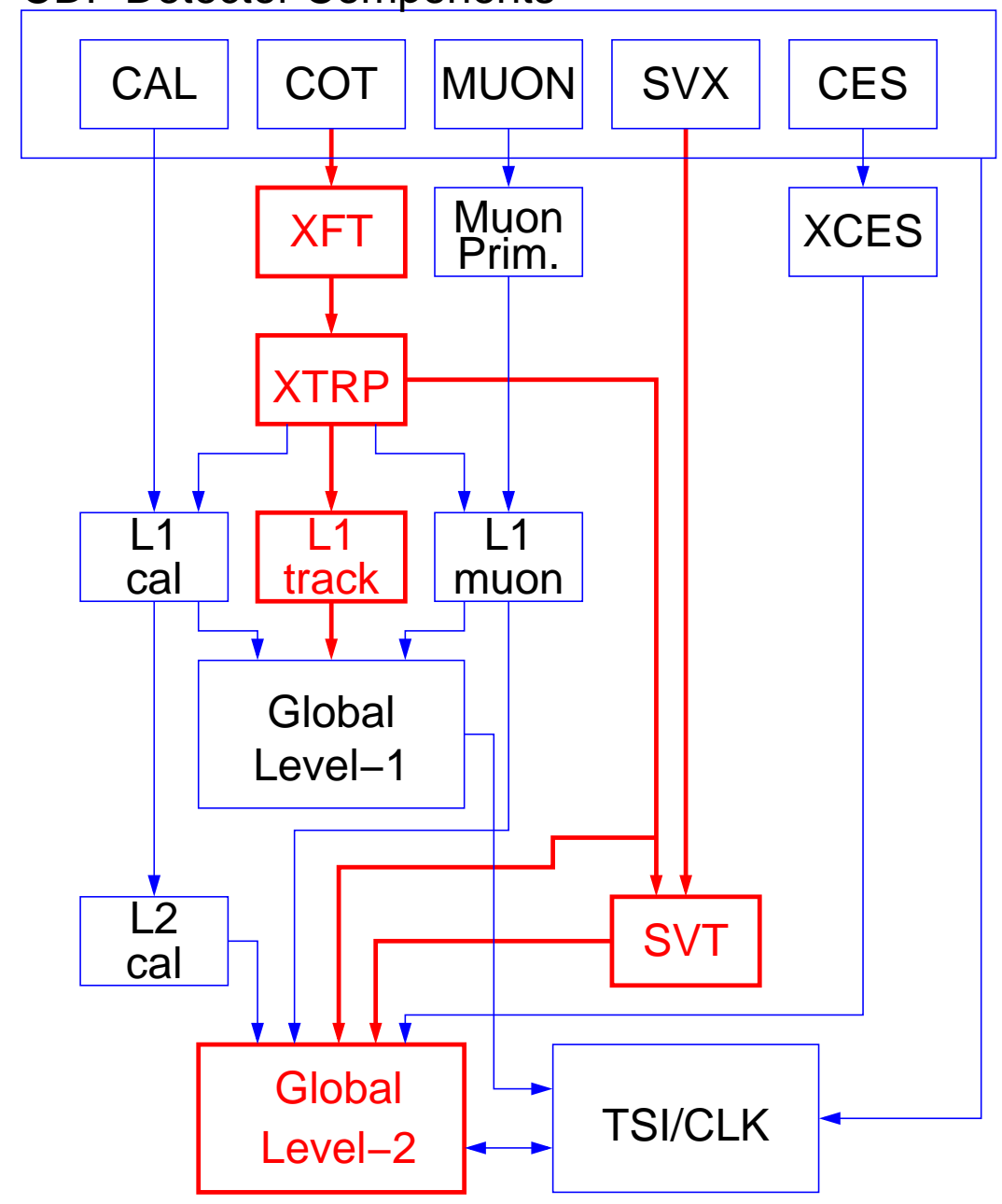

Figure 3-11: Diagram of the different trigger paths at Level 1 and 2. The data flow for the track trigger paths is outlined in red.

\subsubsection{Level 1 Trigger}

The Level 1 trigger decision is based on the information from the eXtremely Fast Tracker (XFT) [39]. This device examines the hit information of the COT in wedges of 15 degrees. It reports the measurement of the track $p_{T}$ and $\varphi_{6}$, the angle of the transverse momentum at the sixth superlayer of the COT, which is located $106 \mathrm{~cm}$ radially from the beamline. Based on pre-loaded patterns of COT hits, it is capable of recognizing track segments for tracks with $p_{T}>1.5 \mathrm{GeV} / \mathrm{c}^{2}$ in $15^{\circ}$ wedges of the COT. Two tracks are reported from a given $15^{\circ}$ wedge, the two tracks which are closest to the left and right boundary of a given wedge. As mentioned in Section 3.4.3, based 
on the hit information from a single COT cell, assuming that the track comes from the beamline, a rough measurement of the track $p_{T}$ and $\varphi_{6}$ is obtained. This is the information that the XFT device determines per track. An event is accepted at Level 1 if two tracks are found in the event such that they have opposite charge, both tracks have $p_{T}>2 \mathrm{GeV} / \mathrm{c}$, the scalar sum of transverse momenta $p_{T 1}+p_{T 2}>5.5 \mathrm{GeV} / \mathrm{c}$ and the $\varphi$ separation between the tracks at superlayer 6 is $\left|\Delta \varphi_{6}\right|<135^{\circ}$.

\subsubsection{Level 2 Trigger}

At Level 2, rough tracking information from the XFT is combined with SVX-II cluster information by the Silicon Vertex Tracker (SVT) [40]. The goal of the second level of the trigger is to obtain a precise measurement of the track $d_{0}$, and improved measurements of $p_{T}$ and $\varphi_{0}$.

Figure 3-12 shows the principle of SVT operation. As mentioned in Section 3.4.1, the SVX-II is segmented into 12 wedges in $\varphi$ and three mechanical barrels in $z$. The SVT makes use of this symmetry and does tracking separately for each wedge and barrel. Tracks which cross wedge and barrel boundaries are only reconstructed under certain circumstances, which are explained in Section 6. An SVT track starts with a two dimensional XFT "seed". The XFT measurement is extrapolated into the SVXII, forming a "road". Clusters of charge on the inner four $r-\varphi$ layers of the given wedge have to be found inside this road. The silicon cluster information and the XFT segment information are fed into a linearized fitter which returns the measurements of $p_{t}, \varphi_{0}$ and $d_{0}$ for the track.

As shown in Figure 3-13, the track impact parameter resolution is about $35 \mu \mathrm{m}$ for tracks with $p_{T}>2 \mathrm{GeV} / \mathrm{c}$. The width of the Gaussian fit for the distribution of track impact parameters in Figure 3-13 is $47 \mu \mathrm{m}$. This width is a combination of the intrinsic impact parameter resolution of the SVT measurement, and the transverse intensity profile of the interaction region. The region profile is roughly circular in the transverse plane and can be approximated by a Gaussian distribution with $\sigma \sim 35 \mu \mathrm{m}$. The intrinsic SVT resolution is obtained by subtracting the beamline width from the width of the $d_{0}$ distribution in quadrature. The Level 1 trigger conditions are 


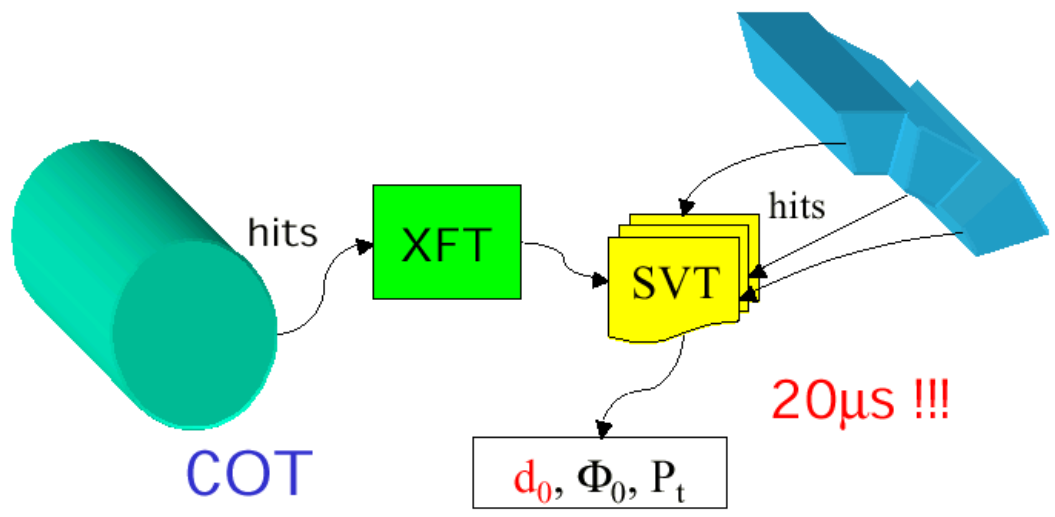

Figure 3-12: SVT principle of operation

confirmed with the improved measurements of $p_{T}$ and $\varphi_{0}$. An event passes Level 2 selection if there is a track pair reconstructed in the SVT such that the tracks have opposite charge, each track has $p_{T}>2.0 \mathrm{GeV} / \mathrm{c}$ and $120 \mu \mathrm{m}<\left|\mathrm{d}_{0}\right|<1 \mathrm{~mm}$. The vertex of the track pair has to have $L_{x y}>200 \mu \mathrm{m}$ with respect to the beamline.

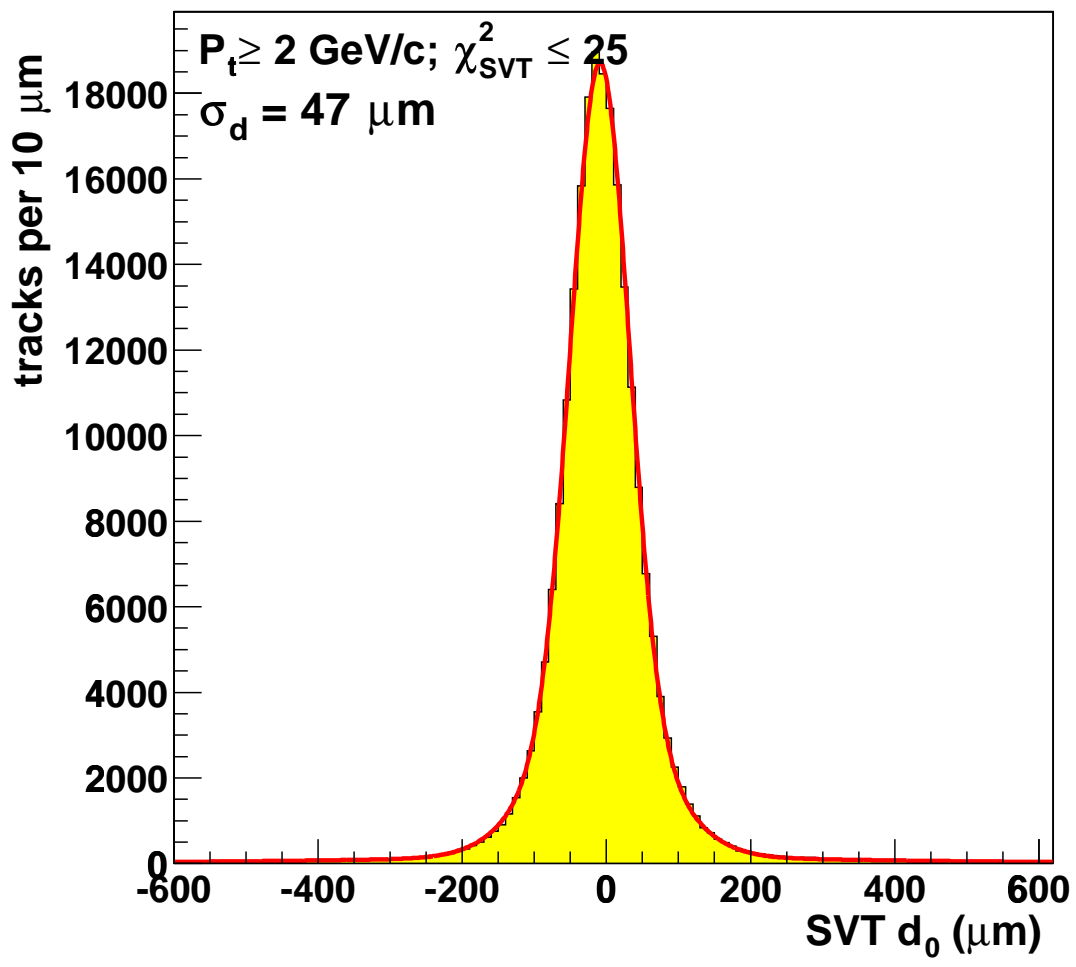

Figure 3-13: SVT impact parameter resolution 


\subsubsection{Level 3 Trigger}

The third level of the trigger system is implemented as a PC farm. Every CPU in the farm provides a processing slot for one event. With roughly 300 CPU's, and a input rate of roughly $300 \mathrm{~Hz}$, this allocates approximately 1 second to do event reconstruction and reach a trigger decision.

Figure 3-14 shows the implementation of the Level-3 farm. The detector readout from the Level 2 buffers is received via an Asynchronous Transfer Mode (ATM) switch and distributed to 16 "converter" node PC's, shown in Figure 3-14 in light blue. The main task of these nodes is to assemble all the pieces of the same event as they are delivered from different subdetector systems through the ATM switch. The event is then passed via an Ethernet connection to a "processor" node, which there are about 150 in the farm and are shown in Figure 3-14 in green. Each processor node is a separate dual-processor PC. Each of the two CPU's on the node process a single event at a time. The Level 3 decision is based on near-final quality reconstruction performed by a "filter" executable. If the executable decides to accept an event, it is then passed to the "output" nodes of the farm, which are shown in Figure 3-14 in yellow. These nodes send the event onward to the Consumer Server / Data Logger (CSL) system for storage first on disk, and later on tape.

For most of the data used for this analysis, full COT tracking was being used to reconstruct tracks. The measurements of $p_{T}, z_{0}, \varphi_{0}$ and $\cot \theta$ from the COT are combined with the $d_{0}$ measurement from the SVT to create a further improved track. The Level 1 and Level 2 trigger conditions (including the requirement on the twotrack vertex $L_{x y}$ ) are repeated at Level 3 using improved track measurements. For later data, which is not used in this analysis, full SVX-II tracking is available, and the trigger conditions are repeated using a combined COT/SVX-II fit of the track helices. 


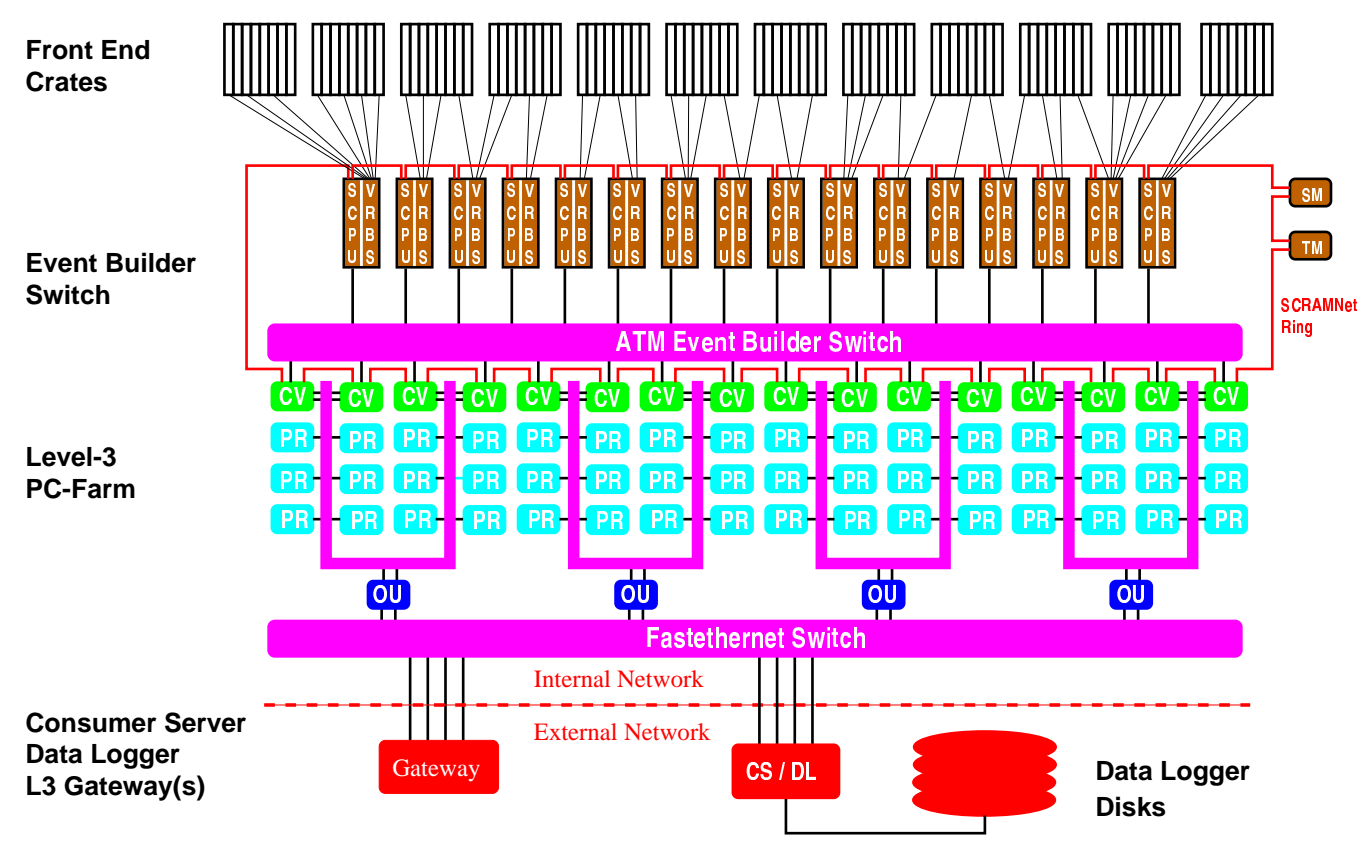

Figure 3-14: Event building and Level 3 operating principle: data from the front end crates is prepared by Scanner CPU's (SCPU) and fed into the ATM switch (purple). On the other side of the switch, converter nodes (green) assemble events and pass them to processor nodes. Accepted events are passed to output nodes (dark blue) which send them to the Consumer Server and data logging systems (red). 


\section{Chapter 4}

\section{Dataset and Candidate Selection}

The measurement that we focus on relates the rate of reconstructed $B_{s}^{0} \rightarrow D_{s}^{-} \pi^{+}$ decays to that of $B^{0} \rightarrow D^{-} \pi^{+}$decays. Individual and relative rates are modified by changing the conditions under which the data are gathered or the way that particles are reconstructed. In order to make the measurement robust, we only analyze data for which the data taking and trigger conditions are stable. To minimize the differences between reconstruction conditions, we keep the selection requirements similar for the two reconstructed mesons.

The nominal trigger conditions for multibody hadronic $B$ data have been proposed long before Run II started [41, 42]. Detailed studies on Run I minimum bias data and signal Monte Carlo predicted trigger rates and purity. Depending on the instantaneous luminosity of the Tevatron, three scenarios were proposed. The three scenarios differ in the value of the minimum $p_{T}$ requirements that are placed on the individual tracks and the track pair.

During Run II data taking, the trigger path closest to the nominal multibody hadronic trigger is the B_CHARM trigger path. The trigger conditions apply to events accepted by this path are not completely homogeneous throughout the currently available data samples. In particular, the trigger was undergoing heavy modifications and improvements from September 2001 to September 2002, and it was running under much more stable conditions from September 2002 until March 2003. In the first period, roughly $67 \mathrm{pb}^{-1}$ of data were gathered. We refer to this period as the winter 
data sample, or hbot0h sample. In the second period, an additional $53 \mathrm{pb}^{-1}$ of data were gathered. We refer to this period as the summer data sample, or hbot1i data sample. The distinction between winter and summer samples comes from the fact that the first sample was available for the winter conferences, and the other became available for the summer conferences of 2003.

In this chapter, we describe the procedures applied to the data in order to obtain a sample in which the relative rate of $B_{s}^{0}$ and $B^{0}$ meson production is understood and reproduced with detailed simulation. In the first step, events that pass only one trigger path are selected. This can be simulated in detail. The second step is finding a set of selection requirements that does not bias the ratio of $B_{s}^{0}$ to $B^{0}$ mesons found in the sample, but still produces a clean signal of $B$ meson decays.

\subsection{Good Runs}

To ensure that data taking conditions are homogeneous for the different detector components, the CDF-II collaboration uses the concept of good runs. For this analysis, it is relevant to have homogeneous running conditions between the tracking systems and the track-based triggers in order to have stable rates which are reproducible by Monte Carlo simulation. Therefore, we only use good runs for our analysis. A run is marked as a good run if the shift operators, offline production operators and subdetector experts sign off that the data taking conditions for all relevant subdetectors are good enough that the data can be analyzed in a reliable fashion. Good run criteria are suggested by the CDF-II data validation group.

In practice, the shift takers and subdetector experts mark runs as good or bad by setting appropriate bits to true or false in the CDF-II Run Database [43]. According to the recommendations of the data validation group for B meson physics analyses, the following boolean values are required to be true for a run to be considered a "Good Run": RUNCONTROL_STATUS, SHIFTCREW_STATUS, OFFLINE_STATUS, CLC_STATUS, L1T_STATUS, L2T_STATUS, L3T_STATUS, SVT_STATUS, SVX_STATUS, COT_OFFLINE, and SVT_OFFLINE. 
Runs in which there are problems with the detector setup are quickly stopped, so an indication of problems with a run is the fact that it is very short. The first bit to check is RUNCONTROL_STATUS, which is set to true if there are 100 million live Tevatron clock cycles, 10000 Level 1 accepts, 1000 Level 2 accepts and at least 1 nb $^{-1}$ of luminosity in the run. Any run shorter than that was most likely aborted because of technical problems with the detector setup.

The SHIFTCREW_STATUS and OFFLINE_STATUS bits are set by the data acquisition shift person, who confirms that no temporary, undocumented (TEST) trigger tables were used in the gathering of the data, and the offline shift crew, which confirms that the data gathered during this run has been sent through offline production without problems or production executable crashes.

The CLC_STATUS bit will be set if the high voltage was on for the Čerenkov Luminosity Counters during the run, and luminosity and beam monitor plots are reasonable. This guarantees good quality of the luminosity measurement done offline using the CLC subdetector. In our analyses, we use luminosity measurements from this subdetector to correctly weight our Monte Carlo samples. This is a minor correction to the effects we extract from the Monte Carlo, and a rough measurement of the luminosity is sufficient.

L1T_STATUS and L2T_STATUS bits are set if Level 1 and Level 2 trigger monitoring plots agree with templates provided to the shift crew. The L3T_STATUS bit is set if the rate of SVX data corruption errors is less than $1 \%$ and the run number for the run is correctly set. If the SVT online beam position subtraction is done correctly and the SVT occupancy is within limits set by experts, the SVT_STATUS will be set. If, in addition, the $D^{0}$ and $D^{*+}$ yields are within an expected range, the SVT_OFFLINE bit will be set, too. The SVX_STATUS bit will be set on if the SVX high voltage is on. For the COT_OFFLINE bit to be set, the integrated luminosity of the run has to be larger than $10 \mathrm{nb}^{-1}$ and the number of bad COT channels has to be less than $1 \%$. The first requirement is a slightly tighter requirement on the run length, and the second guarantees good COT performance for tracking. 


\subsection{Trigger Paths}

Before going into more detail, we make a few introductory remarks about trigger paths, versions and volunteers. Each trigger path has a well defined sequence of Level 1, Level 2 and Level 3 triggers. As mentioned before in Section 3.8, requiring that an event is accepted through a well defined trigger path eliminates volunteer events. In the dataset relevant to our analysis, some of the triggers underwent modifications and development. To keep track of such effects, each trigger has an independent version number. The higher the trigger level, the more often its version changes, because all higher level triggers update their versions when a corresponding lower level trigger version changes, to uniquely identify the sequence applied. A trigger at any level is uniquely specified by its name, which also contains the version number.

The trigger and path versions are added to the name separated by an underscore and has the format $\mathrm{v}-\mathrm{X}$ with $\mathrm{X}$ being the version number. In the following sections the version number always refers to the corresponding trigger path unless explicitly mentioned otherwise. In order to have good control over the rates of $B_{s}^{0}$ and $B^{0}$ detection, we will require that all the reconstructed $B$ mesons were gathered through the same path. Later, we simulate this path in Monte Carlo to investigate differences between $B_{s}^{0}$ and $B^{0}$ mesons. We choose the main path for $B$ meson reconstruction, the B_CHARM trigger path. Other paths that $B$ mesons can be gathered through include B_CHARM_LOWPT, B_CHARM_HIGHPT and seven track paths, which we also describe briefly. A detailed discussion on the trigger requirements can be found in Reference [42]. The B_CHARM_LOWPT and B_CHARM_HIGHPT paths are relevant for increasing the total $B$ meson yield, whereas the seven track paths are introduced to reduce combinatorics in decision making in the Level 1 trigger. For comparison, the nominal trigger requirements for the B_CHARM, B_CHARM_LOWPT and B_CHARM_HIGHPT trigger paths are listed in Table 4.2.3.

In order to control triggers with high accept rates, prescales are introduced. A trigger prescaled with a factor $N$ will only pass every $N$-th event which satisfies the specified trigger conditions. For some trigger paths in our dataset, the prescale factor 
is set dynamically during run time, based on the available trigger bandwidth and collision luminosity. These triggers are referred to as Dynamically Prescaled triggers and typically have the string DPS embedded in their names. Dynamically prescaled triggers are implemented to make use of all available trigger bandwidth. During collisions, the instantaneous luminosity drops as protons and antiprotons get used in the collisions. This causes unprescaled trigger rates to drop with time. Dynamically prescaled triggers adjust their prescale rate according to the instantaneous luminosity to populate the remaining rate which is not filled out by unprescaled triggers at low luminosities.

\subsubsection{The B_CHARM Trigger Path}

The B_CHARM trigger path is the closest to the original "multi-body hadronic trigger path" [41] proposed for gathering hadronic multi-body decays of $B$ mesons with the SVT. It has been modified slightly with respect to the original proposal. An $L_{x y}$ cut has been added at Level 2 which did not exist in the original proposal. In addition, the trigger is dynamically prescaled so its rate would not overload the trigger system at high luminosities.

Currently, the B_CHARM trigger path has had eleven different versions and three different sets of prescales. In the run range of the hbot0h datasets, the relevant B_CHARM versions are 4 through 11 . Versions 4 through 7 are not prescaled, and versions 8 through 11 have dynamic prescales. In the hbot1i dataset, the relevant versions of the trigger are version 11, DPS_v-1 and DPS_v-2. The default prescale for the prescaled triggers is 1 . The dynamic prescale range can change the prescale factor from 1 to 10. The corresponding luminosities are listed in Table 4.1. We introduce the term of effective luminosity for a trigger. This is the luminosity divided by the average prescale factor. For dynamically prescaled triggers, the event yield will follow the effective luminosity, not the total luminosity. This distinction is relevant when weighting Monte Carlo samples representing different run ranges. 


\begin{tabular}{l|l|c|c|c|c} 
Path & Dataset & LowRun & HighRun & $\mathcal{L}\left(\mathrm{pb}^{-1}\right)$ & Eff. $\mathcal{L}\left(\mathrm{pb}^{-1}\right)$ \\
\hline \hline B_CHARM_v-4 & hbot0h & 138766 & 139369 & 0.414 & 0.414 \\
B_CHARM_v-5 & hbot0h & 139239 & 140999 & 0.908 & 0.908 \\
B_CHARM_v-6 & hbot0h & 140685 & 149821 & 7.864 & 7.864 \\
B_CHARM_v-7 & hbot0h & 146623 & 152898 & 13.156 & 13.156 \\
B_CHARM_v-8 & hbot0h & 150708 & 152949 & 8.693 & 7.409 \\
B_CHARM_v-9 & hbot0h & 152413 & 156487 & 35.247 & 34.902 \\
B_CHARM_v-10 & hbot0h & - & - & 0 & 0 \\
B_CHARM_v-11 & hbot0h & 154470 & 156464 & 0.824 & 0.815 \\
B_CHARM_v-11 & hbot1i & 159603 & 161324 & 14.794 & 14.550 \\
B_CHARM_DPS_v-1 & hbot1i & 161327 & 163113 & 33.209 & 31.688 \\
B_CHARM_DPS_v-2 & hbot1i & 163117 & 164200 & 4.076 & 3.993
\end{tabular}

Table 4.1: Luminosities corresponding to the different versions of the B_CHARM trigger path.

\section{Level 1}

At Level 1, the B_CHARM trigger requires a pair of XFT tracks with opposite charge, and hits in at least 4 XFT layers for each track. Both tracks have $p_{T}>2.04 \mathrm{GeV} / \mathrm{c}$ as measured in the XFT. For the track pair, there is a requirement on the scalar sum of transverse momenta, $\sum p_{T}>5.5 \mathrm{GeV} / \mathrm{c}$ and the opening angle at superlayer 6 of the COT, $0<\Delta \varphi_{6}<135^{\circ}$.

The algorithm of the Level 1 decision and its parameters have not changed for different versions of the trigger relevant to this analysis, which are listed in Table 4.1. The name of the trigger used at Level 1 is L1_TWO_TRK2_OPPQ_DPHI135_SUMPT5.5_v-2 From B_CHARM version 6 onward this Level 1 trigger name changes, introducing specific options. The options specify the values of trigger requirements (like track $p_{T}$ ) and allow for the tuning of these values. Even though this functionality is implemented, the specific options are the same as the previously hardcoded values.

Versions 4 through 7 of the B_CHARM path have a hardcoded unit prescale factor for the Level 1 trigger. Versions 8 and higher have dynamic prescales; the prescale factor can be varied from 1 to 5 , and the default value is 1 . In versions 9 through 11 of the B_CHARM path, the range of the prescale factor is extended to 10 , while the default value is still 1 . The distribution of luminosities for different versions of the 
trigger are shown in Table 4.1.

\section{Level 2}

The Level 2 trigger is not quite as homogeneous, as trigger requirements actually changed during data taking. The two periods of data taking are B_CHARM versions 4 through 6, and B_CHARM versions 7 and higher.

In the first period of data taking, the trigger requires two SVT tracks. Each track is required to have SVT fit $\chi^{2}$ less than 25 , impact parameter as measured in the SVT between $100 \mu \mathrm{m}$ and $1 \mathrm{~mm}$ and $p_{T}>2 \mathrm{GeV} / \mathrm{c}$. For this range of trigger paths versions (4-6), the opposite charge, opening angle, and $\sum p_{T}$ requirements are not confirmed at Level 2. They are taken over from Level 1. The trigger name used in this period is L2_TWO_TRK2_D100_v-2.

In the second period of data taking, the Level 2 trigger makes additional requirements. It requires two tracks with opposite charge. Each track is required to have SVT fit $\chi^{2}<25$, impact parameter as measured in the SVT between $120 \mu \mathrm{m}$ and $1 \mathrm{~mm}$, and $p_{T}>2.0 \mathrm{GeV} / \mathrm{c}$. The track pair is required to have an opening angle which satisfies $2^{\circ}<\Delta \varphi_{0}<90^{\circ}$, the scalar sum of transverse momenta satisfies $\sum p_{T}>5.5 \mathrm{GeV} / \mathrm{c}$, and the displacement of the two-track vertex is required to be $L_{x y}>200 \mu \mathrm{m}$. The trigger name for this period is L2_B_CHARM_v-1.

\section{Level 3}

The Level 3 executable matches SVT tracks to COT tracks by requiring proximity in curvature and $\varphi_{0}$ between tracks reconstructed offline and in the SVT. The track $d_{0}$ is set to the SVT measurement, and the other four track parameters are taken from the COT measurement of the track. Pairs of these hybrid tracks are then subjected to the Level 3 requirements.

The cuts on the individual tracks did not change much between trigger paths versions 3 and 11 . An event has to have at least two tracks such that each track has $p_{T}>2 \mathrm{GeV} / \mathrm{c},|\eta|<1.2$ and impact parameter between $120 \mu \mathrm{m}$ and $1 \mathrm{~mm}$.

In versions 4 and later, the upper cut on the track impact parameter $(<1 \mathrm{~mm})$ 
is implemented, while it does not exist for version 3. For version 4 of the Level 3 algorithm, the tracks in the pair are required to have opposite charge. The track pair has to have an opening angle $2^{\circ}<\Delta \varphi<135^{\circ}$. The distance between the two tracks along the beam axis is required to be $\left|\Delta z_{0}\right|<5 \mathrm{~cm}$ and scalar $\sum p_{T}>5.5 \mathrm{GeV} / \mathrm{c}$. Versions 5 and later of the B_CHARM trigger path include the two-track $L_{x y}$ cut: $L_{x y}>$ $200 \mu \mathrm{m}$.

\subsubsection{The B_CHARM_LOWPT Trigger Path}

The B_CHARM_LOWPT trigger path complements the main B_CHARM trigger path. The first two versions of the trigger path are pertinent to the hbot0h dataset. The idea of this trigger is to populate the remaining bandwidth for the bottom triggers by making less stringent trigger requirements, and dynamically changing the prescale factor as the instantaneous luminosity drops during the store. Therefore, the trigger requirements for the lowpt path are looser than those of the B_CHARM: the two tracks are not required to have opposite charge, and the requirement on the scalar sum of the track $p_{T}$ is not applied either.

The luminosities covered by the two different versions of the trigger paths are quoted in Table 4.2. Note that the total luminosity covered does not have to match the total luminosity of good runs, since these triggers were not in use at the beginning of data taking in some stores, due to high luminosities which would saturate the trigger bandwidth. In addition, the B_CHARM_LOWPT trigger is prescaled most of the time, so the difference between the effective luminosity and the total luminosity are much more significant than in the case of the B_CHARM trigger path.

\section{Level 1}

At Level 1, the decision is made by the L1_TWO_TRK2_DPHI90-DPS_v-1 algorithm which requires two tracks, each with hits in 4 XFT layers. Each track is required to have $p_{T}>2.04 \mathrm{GeV} / \mathrm{c}$. The pair opening angle at superlayer 6 is required to be $\Delta \phi_{6}<90^{\circ}$. There is no requirement on $\sum p_{T}$ or the charge of the tracks. The Level 1 trigger 


\begin{tabular}{|c|c|c|c|c|c|}
\hline Path & Dataset & LowRun & HighRun & $\mathcal{L}\left(\mathrm{pb}^{-1}\right)$ & Eff. $\mathcal{L}\left(\mathrm{pb}^{-1}\right)$ \\
\hline "B_CHARM_LOWPT_v-1 & hbot0h & 150708 & 15156487 & 43.940 & 21.527 \\
\hline B_CHARM_LOWPT_v-2 & hbot0h & 154470 & 156464 & 0.824 & 0.544 \\
\hline B_CHARM_LOWPT_v-2 & hbot1i & 159603 & 161324 & 14.794 & 11.362 \\
\hline B_CHARM_LOWPT_DPS_v-1 & hbot1i & 161327 & 163113 & 33.209 & 21.722 \\
\hline B_CHARM_LOWPT_DPS_v-2 & hbot1i & 163117 & 164200 & 4.076 & 3.478 \\
\hline
\end{tabular}

Table 4.2: Highest and lowest good run numbers and luminosities covered by different versions of the B_CHARM_LOWPT trigger path.

decision is prescaled by a factor ranging from 1 to 250 , with the default value being 250.

\section{Level 2}

At Level 2, the decision is based on the L2_B_CHARM_LOWPT_v-1 trigger algorithm. It requires two tracks with SVT fit $\chi^{2}<25$; each track is required to have $p_{T}>2 \mathrm{GeV} / \mathrm{c}$ and $120 \mu \mathrm{m}<\left|d_{0}\right|<1 \mathrm{~mm}$. The track pair is required to have an opening angle $\Delta \phi_{0}<90^{\circ}$ and displacement $L_{x y}>200 \mu \mathrm{m}$. Again, there is no requirement on track charge or $\sum p_{T}$.

\section{Level 3}

The two versions of the trigger path differ in details from the Level 3 filter implementation, but have the same trigger requirements. They both require two COT tracks matched to SVT tracks. Each track is required to have $p_{T}>2.0 \mathrm{GeV} / \mathrm{c}$ and $120 \mu \mathrm{m}<\left|d_{0}\right|<1 \mathrm{~mm}$. The track separation along the $z$ axis is required to be $\left|\Delta z_{0}\right|<5 \mathrm{~cm}$. The track pair opening angle is required to be $2^{\circ}<\Delta \varphi_{0}<90^{\circ}$ and the scalar sum of momenta is required to be $\sum p_{T}>4.0 \mathrm{GeV} / \mathrm{c}$.

\subsubsection{The B_CHARM_HIGHPT Trigger Path}

The B_CHARM_HIGHPT trigger path has been added as a trigger path with lower yield that would not be prescaled during data taking. This guarantees a non-prescaled 


\begin{tabular}{l|l|c|c|c|c} 
Path & Dataset & LowRun & HighRun & $\mathcal{L}\left(\mathrm{pb}^{-1}\right)$ & Eff. $\mathcal{L}\left(\mathrm{pb}^{-1}\right)$ \\
\hline \hline B_CHARM_HIGHPT_v-1 & hbot0h & 150708 & 152949 & 8.693 & 8.483 \\
B_CHARM_HIGHPT_v-2 & hbot0h & 152413 & 156487 & 35.247 & 35.247 \\
B_CHARM_HIGHPT_v-3 & hbot0h & - & - & 0 & 0 \\
B_CHARM_HIGHPT_v-4 & hbot0h & 154470 & 156464 & 0.824 & 0.824 \\
B_CHARM_HIGHPT_v-4 & hbot1i & 159603 & 163113 & 48.003 & 48.003 \\
B_CHARM_HIGHPT_v-5 & hbot1i & 163117 & 164200 & 4.076 & 4.076
\end{tabular}

Table 4.3: Highest and lowest good run numbers and luminosities covered by different versions of the B_CHARM_HIGHPT trigger path.

trigger path that can be used in a straight-forward manner for cross section measurements.

Currently, it has five different path versions. The trigger algorithm is similar to the B_CHARM trigger, but with higher $p_{T}$ and $\sum p_{T}$ requirements to lower the rate.

The run ranges, active and scaled luminosities for the B_CHARM_HIGHPT trigger are listed in Table 4.3. The discrepancy between active and effective luminosity for version 1 comes from a single run (152615) where the number of "prescaled" events in the database is 0 , while the number of unprescaled events for this same path is around 60 million. This is probably a database error and should be irrelevant for all practical purposes.

\section{Level 1}

At Level 1, the selection is performed by the algorithms L1_TWO_TRK2.2_OPPQ_ DPHI135-SUMPT6_v-1 and L1_TWO_TRK2 .5_OPPQ_DPHI135-SUMPT6.5_v-1 corresponding to B_CHARM_HIGHPT path version 1 and all higher versions, respectively. Only a small fraction of the data has been taken with version 1 of the trigger path. Both triggers require two tracks with hits in at least 4 XFT layers. The triggers require that both tracks have $p_{T}>2.23 \mathrm{GeV} / \mathrm{c}$ and $p_{T}>2.46 \mathrm{GeV} / \mathrm{c}$, respectively. The track pair scalar sum of momenta is required to be $\sum p_{T}>6.0 \mathrm{GeV} / \mathrm{c}$ and $\sum p_{T}>6.5 \mathrm{GeV} / \mathrm{c}$, respectively. Both triggers require that the pair opening angle at superlayer 6 be $\Delta \varphi_{6}<135^{\circ}$. 


\section{Level 2}

Similar changes are seen at Level 2 of the trigger. The event selection is performed by the algorithms L2_B_CHARM_HIGHPT_v-1 and L2_B_CHARM_HIGHPT_v-2 for trigger path versions 1 and all higher versions, respectively. The triggers require two tracks with opposite charge. Each track is required to have SVT $\chi^{2}<25$ and $120 \mu \mathrm{m}<$ $\left|d_{0}\right|<1 \mathrm{~mm}$. The two different versions of the trigger require $p_{T}>2.25 \mathrm{GeV} / \mathrm{c}$ and $p_{T}>2.5 \mathrm{GeV} / \mathrm{c}$ respectively. The track pair opening angle is required to be $2^{\circ}<\Delta \varphi<90^{\circ}$ and the displacement of the two-track vertex is required to be $L_{x y}>200 \mu \mathrm{m}$. Version 1 of the trigger requires the scalar sum $\sum p_{T}>6.0 \mathrm{GeV} / \mathrm{c}$, whereas version 2 requires $\sum p_{T}>6.5 \mathrm{GeV} / \mathrm{c}$.

\section{Level 3}

At Level 3, the implementation of the filter may change from version to version, but the trigger requirements stay the same. The filter requires that there are at least two COT tracks in the event matched to SVT tracks. Each track is required to have $120 \mu \mathrm{m}<\left|d_{0}\right|<1 \mathrm{~mm},|\eta|<1.2$ and $p_{T}>2.0 \mathrm{GeV} / \mathrm{c}$. The tracks have to have opposite charge. The track pair is required to have an opening angle $2^{\circ}<\Delta \varphi_{0}<90^{\circ}$, separation along the $z$ axis $\left|\Delta z_{0}\right|<5.0 \mathrm{~cm}$ and $\sum p_{T}>5.5 \mathrm{GeV} / \mathrm{c}$.

\subsubsection{Seven Track Trigger Paths}

The seven track backup trigger has been designed to accept events in which at least seven tracks pass the Level 1 transverse momentum threshold. Analyzing these events in Level 1 and later in the SVT requires a significant amount of time. Since it is quite likely that the event is anyways interesting, these events are automatically accepted from Level 1 onward.

For cross section or branching ratio analyses these trigger types have to be taken into account since a large fraction of the events passing this trigger would also pass the standard trigger of the B_CHARM family. The problem with the seven track backup

trigger is that B_CHARM events could be promoted into the seven track trigger path 


\begin{tabular}{l|c|c|c} 
Requirement & B_CHARM & B_CHARM_LOWPT & B_CHARM_HIGHPT \\
\hline \hline XFT layers /track & 4 & 4 & 4 \\
L1 track min $p_{T}$ & $2.04 \mathrm{GeV} / \mathrm{c}$ & $2.04 \mathrm{GeV} / \mathrm{c}$ & $2.46 \mathrm{GeV} / \mathrm{c}$ \\
L1 pair opposite Q & yes & no & yes \\
L1 pair min $\sum p_{T}$ & $5.5 \mathrm{GeV} / \mathrm{c}$ & none & $6.5 \mathrm{GeV} / \mathrm{c}$ \\
L1 pair $\Delta \varphi_{6}$ & $<135^{\circ}$ & $<90^{\circ}$ & $<135^{\circ}$ \\
\hline L2 track SVT $\chi^{2}$ & 25 & 25 & 25 \\
L2 track min $p_{T}$ & $2.0 \mathrm{GeV} / \mathrm{c}$ & $2.0 \mathrm{GeV} / \mathrm{c}$ & $2.5 \mathrm{GeV} / \mathrm{c}$ \\
L2 track $\left|d_{0}\right|$ & {$[120 \mu \mathrm{m}, 1 \mathrm{~mm}]$} & {$[120 \mu \mathrm{m}, 1 \mathrm{~mm}]$} & {$[120 \mu \mathrm{m}, 1 \mathrm{~mm}]$} \\
L2 pair opposite Q & yes & $n o$ & yes \\
L2 pair min $\sum p_{T}$ & $5.5 \mathrm{GeV} / \mathrm{c}$ & $4.0 \mathrm{GeV} / \mathrm{c}$ & $6.5 \mathrm{GeV} / \mathrm{c}$ \\
L2 pair $\Delta \varphi_{0}$ & $2^{\circ}<\varphi_{0}<90^{\circ}$ & $2^{\circ}<\Delta \varphi_{0}<90^{\circ}$ & $2^{\circ}<\Delta \varphi_{0}<90^{\circ}$ \\
L2 pair min $L_{x y}$ & $200 \mu \mathrm{m}$ & $200 \mu \mathrm{m}$ & $200 \mu \mathrm{m}$
\end{tabular}

Table 4.4: Nominal trigger requirements for the B_CHARM, B_CHARM_LOWPT and B_CHARM_HIGHPT trigger paths at Level 1 and Level 2.

\begin{tabular}{|c|c|c|c|c|c|}
\hline B_CHARM_ Path & Dataset & LowRun & HighRun & $\mathcal{L}\left(\mathrm{pb}^{-1}\right)$ & Eff. $\mathcal{L}\left(\mathrm{pb}^{-1}\right)$ \\
\hline L1_SEVEN_TRK2_v-8 & $\overline{\text { hbot1i }}$ & 159603 & 163113 & 48.003 & 48.003 \\
\hline L1_SEVEN_TRK2_v-9 & hbot1i & 163117 & 164200 & 4.076 & 4.076 \\
\hline HIGHPT_L1_SEVEN_TRK2_V-5 & hbot1i & 159603 & 163113 & 48.003 & 48.003 \\
\hline HIGHPT_L1_SEVEN_TRK2_v-6 & hbot1i & 163117 & 164200 & 4.076 & 4.076 \\
\hline
\end{tabular}

Table 4.5: High, low run numbers, integrated and effective luminosity for the seven track triggers of the B_CHARM and B_CHARM_HIGHPT trigger path families.

because there are many tracks with $p_{T}>2 \mathrm{GeV}$ in the event. Since an event is either accepted through the B_CHARM path or the seven track path, this would change the acceptance of the B_CHARM trigger. Our Monte Carlo generator only simulates the decay of the signal $B$ meson. It does not reproduce the remaining event structure: fragmentation tracks, multiple collisions or the opposite side $B$ meson, which can cause the promotion of our events into the seven track trigger path. Thus the proper way to deal with events from the seven track path is to perform an emulation of the corresponding trigger path of the B_CHARM family in the offline analysis and accept the events which are accepted through the seven track backup trigger and pass B_CHARM trigger confirmation on the candidate of interest. The run ranges, active and scaled luminosities for the seven track trigger paths are listed in Table 4.5. 


\subsection{Event Selection}

Our goal is to reconstruct fully hadronic $B$ meson decays in a sample pre-selected by the B_CHARM trigger. In the following chapters, we will describe the methods which we use to reconstruct these decays. Our reconstruction is track-based, so we discuss methods of track preparation and track quality requirements first. These requirements reduce the amount of background introduced by mis-reconstructed tracks.

We want to make use of the trigger pre-selection when looking for $B$ mesons. In addition, by correctly confirming the trigger conditions on a given candidate, we remove volunteer candidates from our dataset. Therefore, we require that two of the four tracks in our final state be matched to trigger tracks.

Ultimately in our analysis, we want to extract the number of reconstructed $B$ meson candidates with the smallest relative statistical error. In order to achieve this goal, we implement a procedure which optimizes the statistical significance of the signal, defined as:

$$
\mathcal{S}=\frac{N_{S}}{\sqrt{N_{S}+N_{B}}}
$$

where $N_{S}$ is the number of signal events, and $N_{B}$ is the number of background events.

\subsubsection{Track Preparation}

Because our reconstruction is track based, we take special care to ensure that the tracks used for reconstructing candidates are of best available quality. We therefore apply track quality requirements and do additional preparation of the tracks so that the track parameters their error estimates are more realistic.

Due to misalignments in the tracking systems and, inevitably, hits caused by noise in the tracking systems fake and mis-measured tracks appear in the events. A very simple way to reduce the number of these tracks is to require a minimum number of hits in the drift chamber and silicon detector. We select tracks which have at least two SVX $r$ - $\phi$ hits and have a COT parent. This in itself implies that it will have at least $20 \mathrm{COT}$ axial and $20 \mathrm{COT}$ stereo hits. We also require the track to have an error matrix which can be inverted and a helix fit attached to it. The last two 
are requirements which remove very few tracks which otherwise cause the software to crash. The effect is much less than a per mill and is not correlated with particular types of events.

Monte Carlo studies, as well as studies in data have demonstrated that the error matrix of the COT fit of tracks underestimates the measurement errors. In order to correct for this problem, we start from the COT parent of our track and refit it. Before refitting, we rescale the covariance matrix of the COT track with the following scale factors:

$$
\begin{aligned}
& s(\lambda)=\sqrt{1+p_{\lambda}\left(1+\lambda^{2}\right)^{1.5} / p_{T}^{2}} \quad \text { with } p_{\lambda}=0.580 \\
& s(c)=\sqrt{1+p_{c} / p_{T}^{2}} \quad \text { with } p_{c}=5.33 \\
& s\left(z_{0}\right)=\sqrt{1+p_{z_{0}}\left(1+\lambda^{2}\right)^{1.5} / p_{T}^{2}} \quad \text { with } p_{z_{0}}=0.653 \\
& s\left(d_{0}\right)=\quad \sqrt{1+p_{d_{0}} / p_{T}^{2}} \quad \text { with } p_{d_{0}}=3.01 \\
& s\left(\phi_{0}\right)=\quad \sqrt{1+p_{\phi_{0}} / p_{T}^{2}} \quad \text { with } \quad p_{\phi_{0}}=3.7
\end{aligned}
$$

The factors are applied to the covariance matrix as: $c_{i j}^{\text {new }}=s_{i} s_{j} c_{i j}$, where the indices $i$ and $j$ represent the various parameters. The rescaled COT track is used as a starting point for the refit of the combined COT+SVX track. The refit of the track takes into account energy loss corrections for kaon or pion hypothesis according to the requested species. The refit starts from the silicon hits which have been originally assigned to the track. Silicon hits from the intermediate silicon layer and Layer 00 are dropped because both of those subdetectors are not yet fully aligned ${ }^{1}$. The model for the material description is based on the Kalman track refitting package [44]. The alignment table has to be specified for track refitting. We use here the recommended alignment table "ofotl_prd_read 1000301 GOOD".

\footnotetext{
${ }^{1}$ Recently, better alignments became available for both ISL and L00, which were not available at the time of this analysis.
} 


\subsubsection{Track matching to SVT}

For rate or relative rate analyses it is essential to be able to connect the offline tracks with the online trigger information because only this way volunteer candidates are not considered. Volunteer candidates can be due to various reasons: one would be that only one track of the $B$ decay passes the trigger criteria while the second one is provided by a fake XFT track which got several accidental hits assigned and has thus high probability to have a large impact parameter. Another example is an event triggered by a track from one $B$ meson and another track from the other $B$ meson in the event. All of those cases are undesirable in the analysis of the relative branching ratios since they will not be reproduced by the Monte Carlo and thus the efficiencies will be incorrect. Our Monte Carlo simulation only generates the decay products of exactly one $B$ hadron per event.

The SVT matching algorithm is based on the svtsim [45] package. The track matching algorithm calculates the distance:

$$
\Delta=\sqrt{\frac{\left(C_{\text {offline }}-C_{S V T}-C_{\text {mean }}\right)^{2}}{\sigma_{C}^{2}}+\frac{\left(\phi_{\text {offline }}^{0}-\phi_{S V T}^{0}-\phi_{\text {mean }}^{0}\right)^{2}}{\sigma_{\phi}^{2}}}
$$

where $C_{\text {offline }}$ is the offline measured curvature, $C_{S V T}$ is the curvature measured by the SVT, $\phi_{\text {offline }}^{0}$ and $\phi_{S V T}^{0}$ are the $\phi_{0}$ angles measured by offline and SVT reconstruction, respectively. The values $C_{\text {mean }}, \sigma_{C}$ are the mean and width of the difference between offline and SVT curvature measured in $J / \psi$ signal data [46]. The fits of the difference between SVT and offline measured curvature an $\varphi_{0}$ are depicted in Figure 4-1. The values $\phi_{\text {mean }}^{0}$ and $\sigma_{\phi}$ are the corresponding mean and width of of the $\phi$ difference. The non-zero mean of the $\phi$ difference between SVT and offline tracks is a consequence of different silicon alignments used in offline and SVT reconstruction. Since the $\phi$ and curvature measurements in a track fit are correlated, this means that the curvature difference between offline and SVT will also have a non-zero mean. Offline matching is only done on SVT tracks which have a SVT fit $\chi^{2}$ which is less or equal to 25. To promote an offline track to being matched to an online trigger track, we require that the distance $\Delta$ be less than 95, and that the SVT measurements of 
the $p_{T}$ and $d_{0}$ to comply with: $p_{T}>2 \mathrm{GeV}$ and $120 \mu \mathrm{m}<\left|d_{0}\right|<0.1 \mathrm{~cm}$.

\subsubsection{Trigger Confirmation}

The confirmation of the trigger is a crucial aspect for this analysis since the SVT fit is not obtained from the exact same hits or fitting algorithm as the offline tracks. Differences in SVT and offline quantities are rather subtle and a careful analysis is necessary. To ensure a correct analysis, we save several levels of trigger information. Per event, we record the complete list of trigger bits: Level 1, Level 2 and Level 3.

This information is important for the analysis since we have to be able to assign each event to a particular trigger path. This could also be done on the candidate level but this is not sufficient since the repetition of the trigger on the candidate track does not take into account the effect of prescale factors. The B_CHARM, B_CHARM_LOWPT and B_CHARM_HIGHPT trigger share a large amount of phase space. Consequently, a candidate which passes B_CHARM confirmation might not have been accepted through the B_CHARM path because of the dynamic prescale, but instead got accepted through the B_CHARM_LOWPT or B_CHARM_HIGHPT paths. Independent trigger prescales for different triggers which share phase space require a proper handling of the event trigger bits for any rate or relative rate analysis.

Apart from the event based trigger information, we further store candidate based trigger information. Each track which has been matched with an SVT track is tagged with a bit. Furthermore, trigger pairs according to the B_CHARM_LOWPT, B_CHARM and B_CHARM_HIGHPT trigger algorithms are determined per event and a candidate gets tagged if it contains a trigger pair. Confirming the trigger on the event basis, and requiring that the trigger be confirmed on the decay products of our $B$ meson candidate guarantees that the candidate was not a volunteer, but that it actually caused the event to be accepted by the trigger.

In order to remove volunteer candidates, we require that two of the tracks that make the $B$ meson candidate are matched to an SVT trigger track pair. The following quantities are available for selection: track charges, the linear SVT $p_{T}$ sum, the $\Delta \phi$ angle between the SVT tracks and the $L_{x y}$ as calculated from the SVT quantities 
$p_{T}, d_{0}$ and $\phi_{0}$ of the two tracks. All of those quantities are recorded as they were available online at the time of the trigger decision, thus the real trigger decision can be repeated in our analysis. For yield studies, it is relevant to record if an event and a candidate passed B_CHARM, B_CHARM_LOWPT or B_CHARM_HIGHPT triggers.

To confirm a B_CHARM candidate, we require the two tracks to have opposite charge. Each track is required to have $p_{T}>2.0 \mathrm{GeV}$, linear sum $p_{T}>5.5 \mathrm{GeV}, 2^{\circ}<\Delta \phi<90^{\circ}$ and $L_{x y}>0.02 \mathrm{~cm}$.

A B_CHARM_LOWPT candidate is required to have $p_{T}>2.0 \mathrm{GeV} / \mathrm{c}$ for both SVT tracks. No additional linear $p_{T}$ sum requirement and $L_{x y}>0.02 \mathrm{~cm}$ are placed. The two tracks are not required to have opposite charge. To confirm a B_CHARM_HIGHPT candidate, we require opposite charge between the two tracks, each track $p_{T}>$ $2.5 \mathrm{GeV}$, linear sum $p_{T}>6.5 \mathrm{GeV}, 2^{\circ}<\Delta \phi<90^{\circ}$ and $L_{x y}>0.02 \mathrm{~cm}$.

\subsubsection{Candidate Reconstruction}

When reconstructing candidates, we do not take advantage of any particle identification information. Instead, we only rely on tracking information. For a given decay topology, we try all possible track combinations and reconstruct the mass spectrum from the measured momenta. Long lived particle decays appear as Gaussian peaks in the otherwise smooth mass spectrum.

Candidates for each particle decay are constructed from the bottom up. For example, in the case of the $B_{s}^{0} \rightarrow D_{s}^{-} \pi^{+}, D_{s}^{-} \rightarrow \phi \pi^{-}$decays we start by reconstructing a $\phi \rightarrow K^{+} K^{-}$decay which is used in the next step to make candidates for $D_{s}^{-} \rightarrow \phi \pi^{-}$ decays. Finally those $D_{s}^{-}$decays are used to reconstruct $B_{s}^{0} \rightarrow D_{s}^{-} \pi^{+}$decays. In each step the candidates are subjected to a set of selection requirements which is optimized to quickly reject uninteresting candidates. Charge correlations between tracks are applied first. Candidates with duplicate tracks are removed. Next, the trigger is confirmed: for the $D$ meson decay, one track is required to be matched to a SVT trigger track without loosing any events. For the full $B$ decay, two online trigger tracks are required. The raw mass is calculated based on the track momenta and candidates are only accepted within a loose mass window. A fit of the decay 
topology is performed and the resulting $\chi^{2}$ in the $r-\varphi$ plane, the fitted mass, and the $L_{x y}$ are used to reject candidates. Selection requirements are only applied if, later at the analysis stage, tighter cuts are applied on the same quantity.

The fit of the decay topology introduces constraints which improve measurements of decay particle track parameters. The tracks corresponding to the $D$ meson decay products are required to originate from the same point in space. The $D$ meson momentum is required to point back to the pion from the $B \rightarrow D \pi$ decay. For $B$ meson candidate fits the mass of the $D$ meson is constrained to its PDG value [10]. We use the CTVMFT [47] package to perform the vertex fit of the decay topology.

\subsubsection{Optimization of Selection Requirements}

Our goal is to reconstruct $B$ decays so that the resulting number of signal candidates has the smallest relative statistical error. This ensures a small statistical error on the measurement of relative fractions, and the same selection can be used in later analyses to maximize the significance of a $B_{s}^{0}$ mixing signal.

In this process, we have to be careful not to choose selection requirements which would artificially enhance the $B$ meson yield. The standard way to avoid this is to use a combination of data and Monte Carlo simulated events to optimize selection requirements. A sample of data containing no signal ("sideband"), but kinematically similar, is used to estimate the number of background events after a selection is applied. A weighted Monte Carlo sample is used to estimate the number of background events after the same selection. From the estimated number of signal and background events, the statistical significance is constructed. Selection requirements are varied to optimize the significance.

The main problem with the described optimization in the hadronic $B$ decay channels is finding an unbiased method to test the requirements while optimizing. The mass spectrum of $B$ decays shows large reflection peaks just below the $B$ mass peaks, as seen in Figure 4-2. Therefore, sampling events from the immediate low-mass sideband can not be done easily.

The solution to this problem is to fit the background to an exponential distribu- 
tion away from the signal mass peak and the main reflections, and use the integral of the exponential distribution under the signal region to determine the number of background events. To ensure that the cuts are not biased, we determine the number of signal events from Monte Carlo; the scaling factor between Monte Carlo and data is determined before the cut optimization is done. A more detailed description of the Monte Carlo generation procedure can be found in Section 5.1.

Figure 4-4 depicts how the number of background events, $B$, and the number of signal events, $S$ are determined. The left plot of Figure 4-4 depicts the fit used to determine the number of background events under the peak; an exponential fit is used and it is limited to the range away from the signal and reflections. The exponential fit is extrapolated into the signal region to determine how many background events are in a $\pm 3 \sigma$ range under the signal Gaussian. The number of signal events is determined from signal Monte Carlo which is rescaled to the number of events seen in the mass peak in the data before cut optimization. The exponential fit slightly overestimates the number of background events. This is a consequence of the sample composition discussion in Section 5.2. The non-Gaussian tails in the signal Monte Carlo distribution come from candidates with mismeasured tracks.

Figure 4-5 shows an example of how the optimization process is done on one cut, the $L_{x y}$ of the $B_{s}$ meson. The two variables that are monitored while optimizing cuts are the signal significance $(\sigma=S / \sqrt{S+B})$, as shown in the left plot, and the analysis efficiency, shown on the right. When given a set of points with similar significance, the point with the highest cut efficiency is chosen. While varying one cut, the values of all the other cuts are kept fixed. The optimization process is iterative; the selection requirements are changed to new values at the beginning of every iteration.

The optimization process starts with a set of selection requirements which are chosen based on Monte Carlo distributions to have high $(\sim 90 \%)$ efficiency. The results of using these selection requirements to select the different $B$ mesons are shown in Figure 4-2.

Figure 4-3 depicts a typical decay topology for $B \rightarrow D \pi$ decays. The $B$ meson has a finite lifetime, so its vertex is displaced from the primary vertex. Furthermore, the 
$D$ meson also has a finite lifetime and its decay vertex is displaced from the $B$ decay vertex. The resulting topology has a three-prong vertex and a single displaced track. The following quantities are available to select such decays: $\chi^{2}(r, \varphi)$ of both the $D$ and $B$ meson fits, the transverse momenta $\left(p_{T}\right)$ of both the $D$ and the $B$ meson, the $L_{x y}$ of the $D$ and $B$ meson with respect to the primary interaction vertex, the $L_{x y}$ of the $D$ meson vertex with respect to the $B$ meson vertex, the transverse momentum of the $B$ meson daughter pion, the $\Delta R$ between the $D$ meson and the $B$ daughter pion, and the $B$ meson impact parameter with respect to the primary vertex. We select to use the $\chi^{2}(r, \varphi)$ of the decays for the following reasons. At the time of this analysis, the $z$ alignments in the Silicon are not well understood. Therefore, it is safer to make selection requirements on variables in the transverse plane. Furthermore, in our procedure we reconstruct three-prong vertices, which should have low $\chi^{2}$ in two dimensions, so a cut on the two-dimensional $\chi^{2}$ separates tracks which originate from a common vertex from combinatorial background.

The optimized selection requirements are listed in Table 4.3.5. When reconstructing $B_{s}$ mesons, we require that the invariant mass of the two kaon tracks be between 1013 and $1028 \mathrm{MeV} / \mathrm{c}^{2}$ in addition to these requirements. Figure 4-6 shows the mass spectra for the three $B$ meson decays obtained after optimizing the selection requirements. 

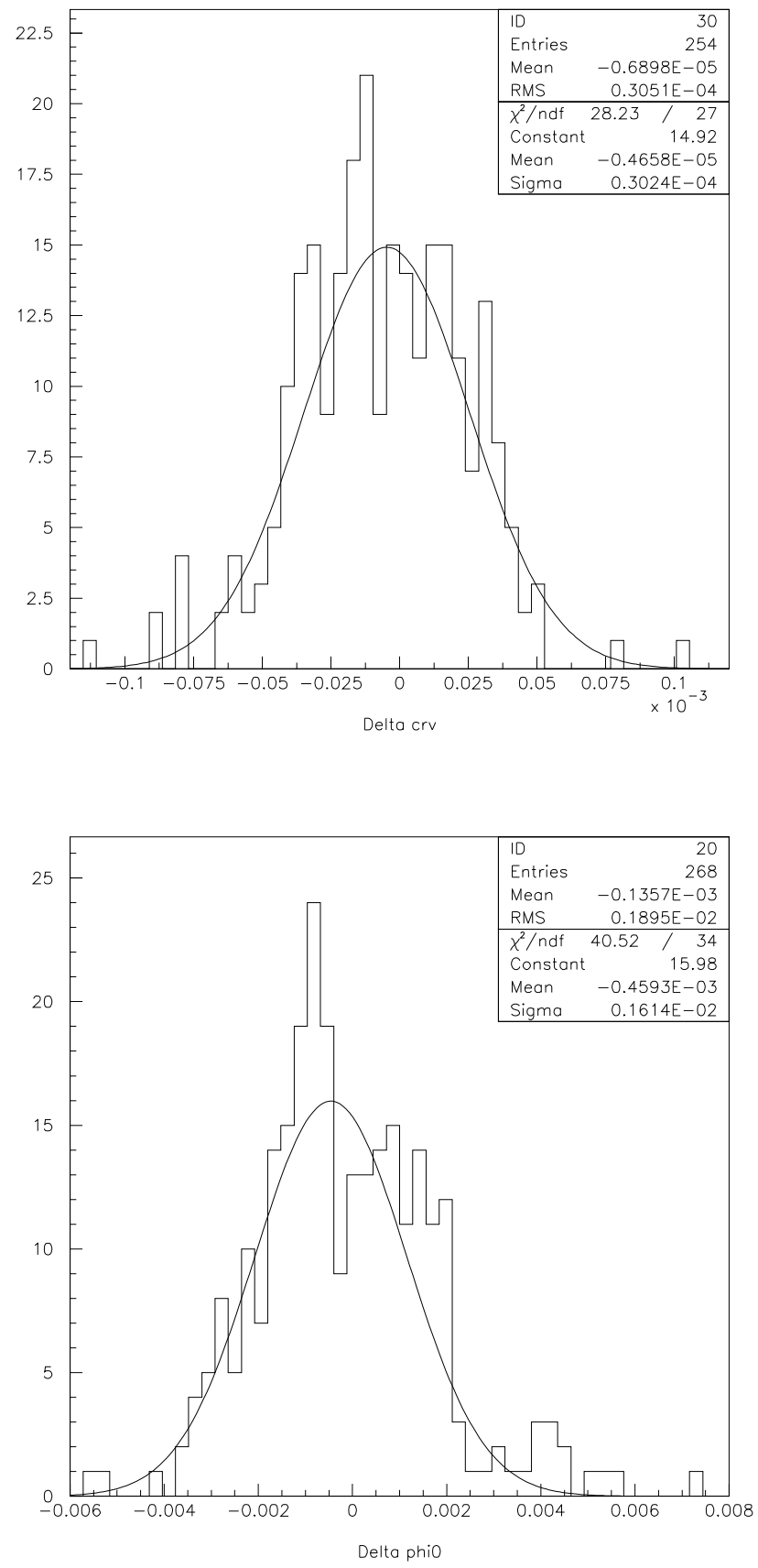

Figure 4-1: Matching SVT quantities to offline quantities. On the left, a plot showing the difference in curvature between SVT and offline measured curvature for sideband subtracted $J / \psi$ data. On the right, the difference between SVT and offline measured $\varphi_{0}$ using the same method. 

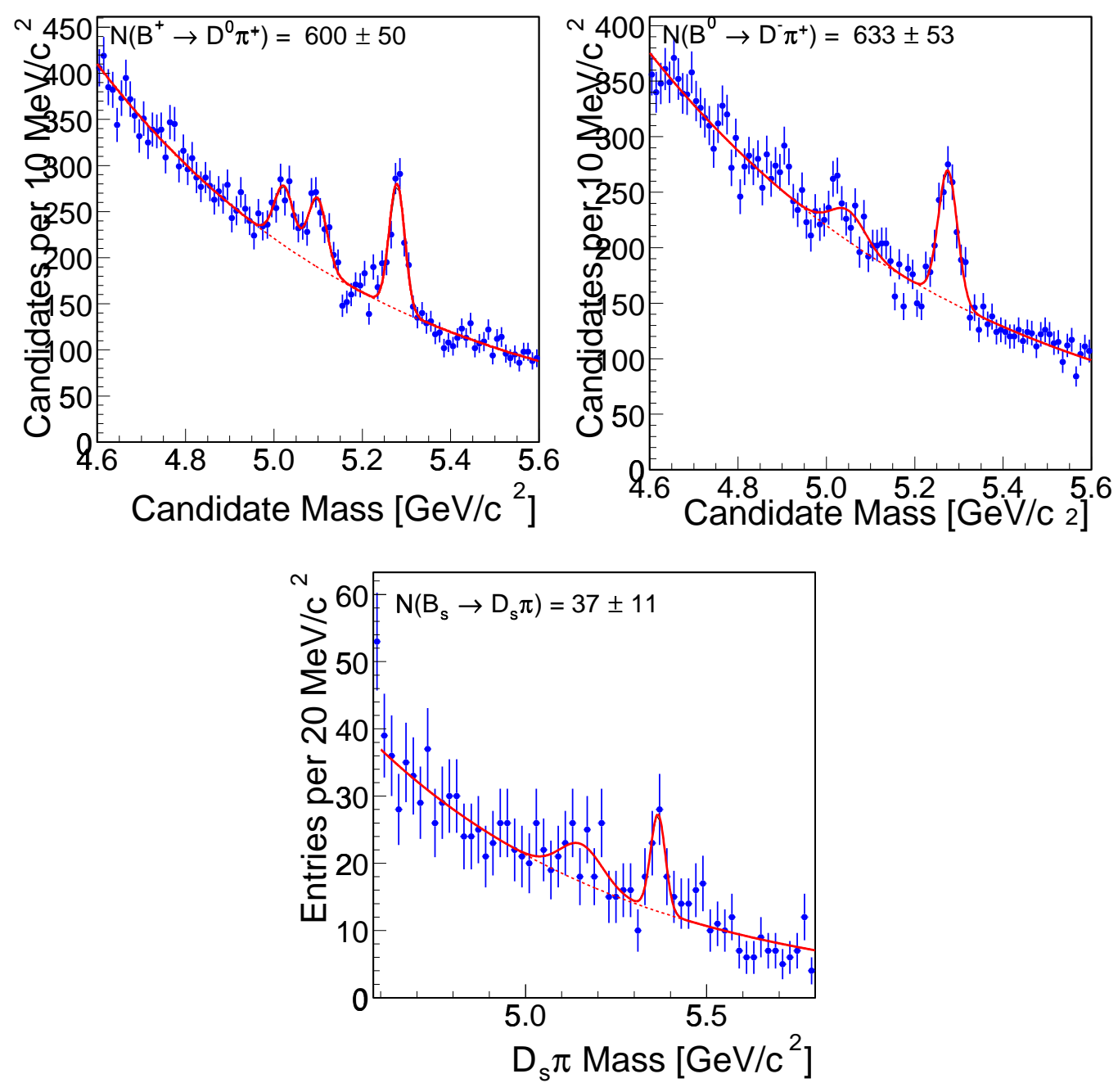

Figure 4-2: Mass spectrum of $B^{+}, B^{0}$ and $B_{s}^{0}$ decays using unoptimized selection requirements on the hbot0h sample. Non-smooth structures are visible below the main signal peaks. 


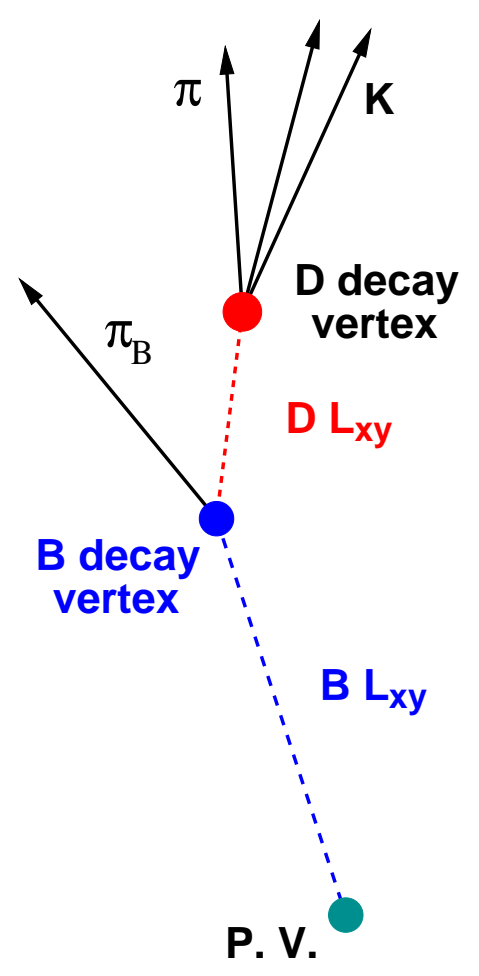

Figure 4-3: Typical topology of a $B \rightarrow D \pi$ decay.
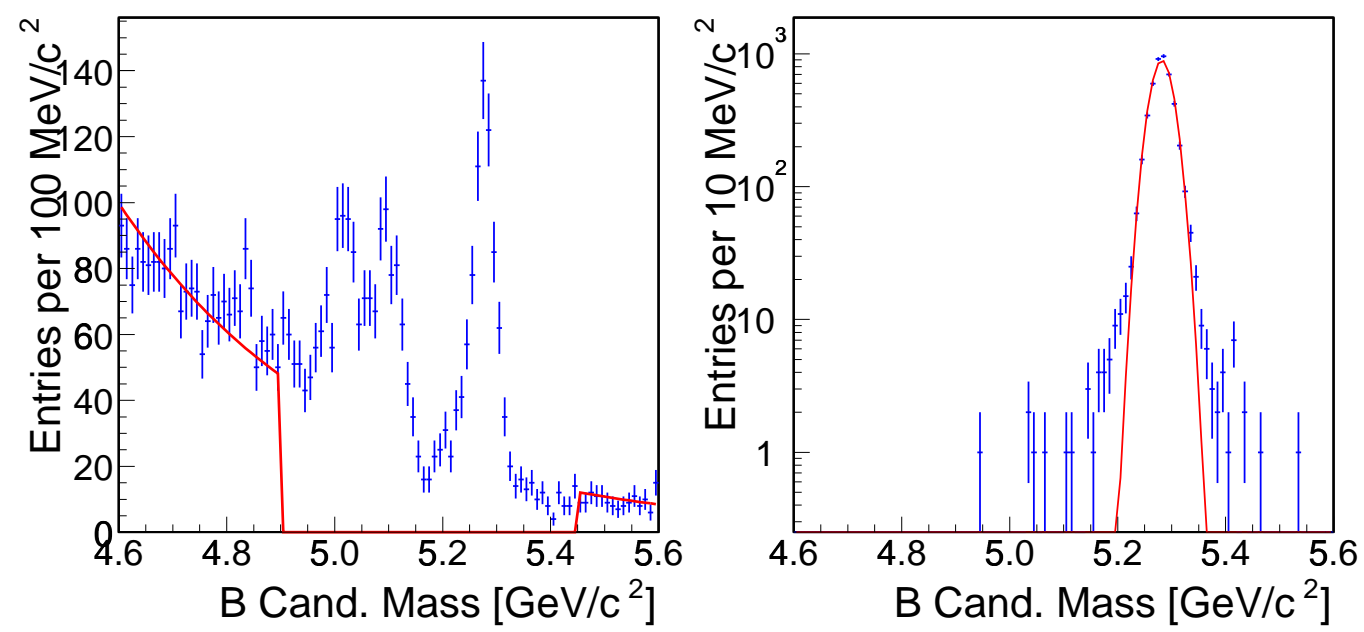

Figure 4-4: Determining the number of background and signal events. On the right, the fit of the background excluding the signal area. On the right, fitting the Monte Carlo simulated signal with a Gaussian. 

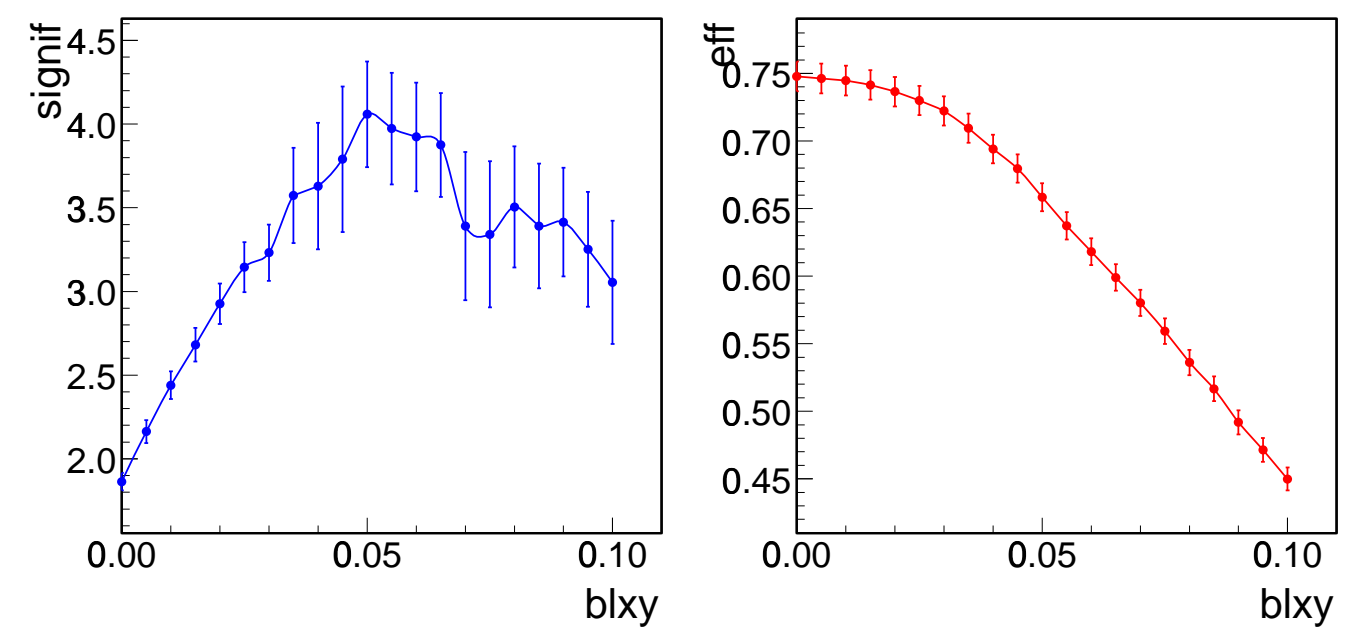

Figure 4-5: An example of variables monitored during the optimization procedure. On the left, the significance of the $B_{s}^{0}$ signal as a function of the $L_{x y}$ selection requirement. On the right, the efficiency of the $L_{x y}$ selection requirement.

\begin{tabular}{l|l|l}
$B_{s}$ & $B^{+}$ & $B^{0}$ \\
\hline \hline$\chi_{r, \varphi}^{2}(D)<14$ & $\chi_{r, \varphi}^{2}(D)<12$ & $\chi_{r, \varphi}^{2}(D)<14$ \\
$\chi_{r, \varphi}^{2}(B)<15$ & $\chi_{r, \varphi}^{2}(B)<13$ & $\chi_{r, \varphi}^{2}(B)<15$ \\
$p_{T}(D)>3.5 \mathrm{GeV} / \mathrm{c}$ & $p_{T}(D)>3.0 \mathrm{GeV} / \mathrm{c}$ & $p_{T}(D)>4.0 \mathrm{GeV} / \mathrm{c}$ \\
$p_{T}(B)>5.5 \mathrm{GeV} / \mathrm{c}$ & $p_{T}(B)>5.0 \mathrm{GeV} / \mathrm{c}$ & $p_{T}(B)>5.5 \mathrm{GeV} / \mathrm{c}$ \\
$L_{x y}(D)>400 \mu \mathrm{m}$ & $L_{x y}(D)>300 \mu \mathrm{m}$ & $L_{x y}(D)>500 \mu \mathrm{m}$ \\
$L_{x y}(B)>400 \mu \mathrm{m}$ & $L_{x y}(B)>300 \mu \mathrm{m}$ & $L_{x y}(B)>300 \mu \mathrm{m}$ \\
$L_{x y}(B \rightarrow D)>-200 \mu \mathrm{m}$ & $L_{x y}(B \rightarrow D)>-200 \mu \mathrm{m}$ & $L_{x y}(B \rightarrow D)>-150 \mu \mathrm{m}$ \\
$\Delta R\left(D, \pi_{B}\right)<1.5$ & $\Delta R\left(D, \pi_{B}\right)<1.5$ & $\Delta R\left(D, \pi_{B}\right)<1.5$ \\
$p_{T}\left(\pi_{B}\right)>1.5 \mathrm{GeV} / \mathrm{c}$ & $p_{T}\left(\pi_{B}\right)>1.5 \mathrm{GeV} / \mathrm{c}$ & $p_{T}\left(\pi_{B}\right)>1.6 \mathrm{GeV} / \mathrm{c}$ \\
$\left|d_{0}(B)\right|<80 \mu \mathrm{m}$ & $\left|d_{0}(B)\right|<80 \mu \mathrm{m}$ & $\left|d_{0}(B)\right|<80 \mu \mathrm{m}$
\end{tabular}

Table 4.6: Optimized selection requirements for reconstructed hadronic $B$ decays 

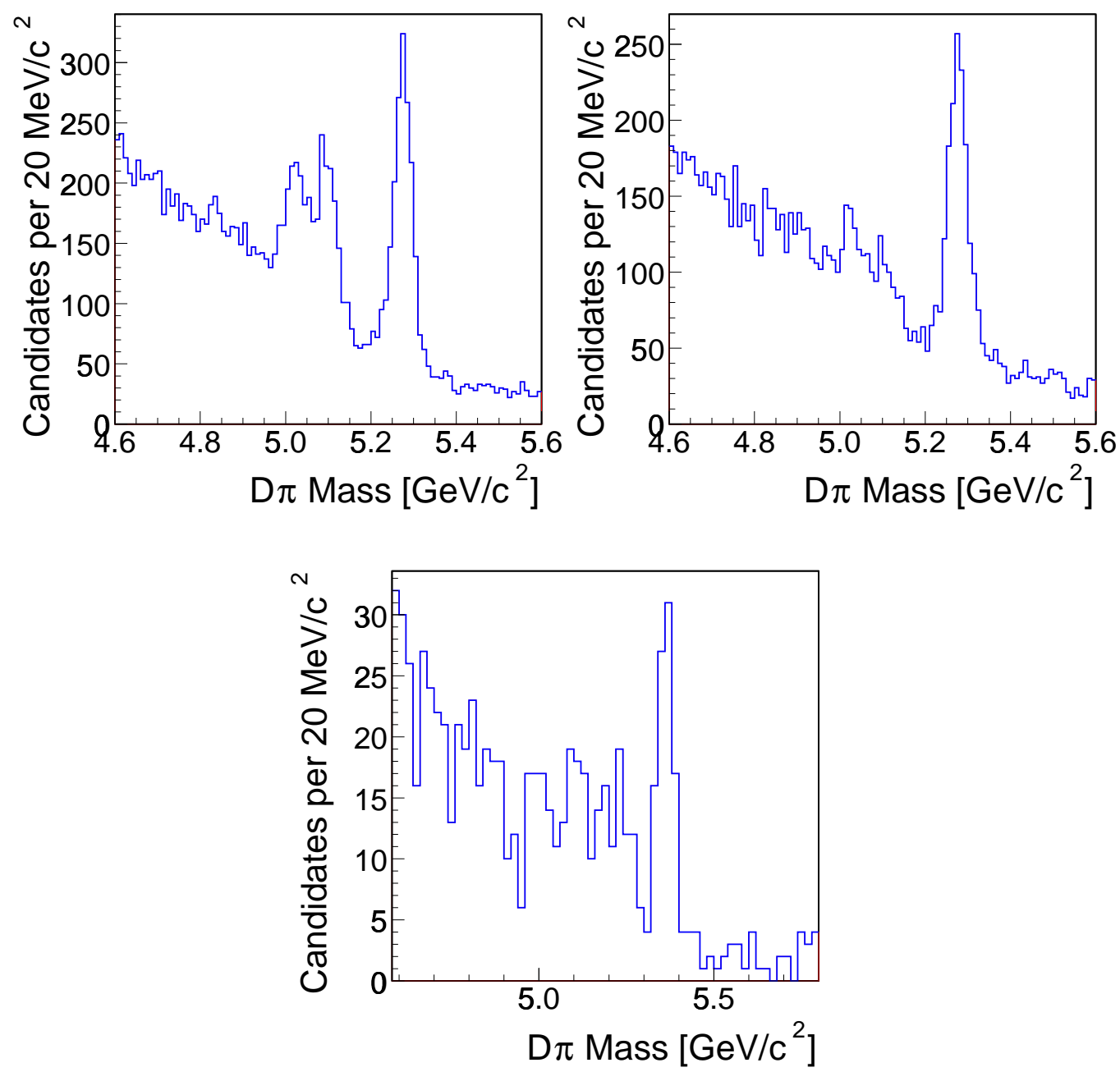

Figure 4-6: Mass spectra of $B^{+}, B^{0}$ and $B_{s}^{0}$ mesons using optimized selection requirements.

\subsection{Summary}

We have reviewed the different trigger paths and versions of multi body hadronic triggers in the hbot $0 \mathrm{~h}$ and hbot1i samples. We find that the trigger conditions are changing for the all trigger paths. For our analysis we choose to measure the rate of $B$ meson decays reconstructed in the B_CHARM trigger path, because we can reliably simulate the trigger effects and changing trigger conditions for this trigger path. Combining input from Monte Carlo and data, we optimize selection requirements to reconstruct $B$ meson decays with high signal significance without biasing the rates. 
The resulting mass spectra have interesting structures and a good $S / B$ ratio. 


\section{Chapter 5}

\section{Rate Analysis}

In the previous chapter, we explain the procedures that guarantee stability and reproducibility of the reconstructed $B$ meson rates. In this chapter we describe the procedures which perform the measurement of the ratio of branching fractions on the sample of reconstructed $B$ mesons.

The reconstructed mass spectra show rich reflection structures. In order to correctly extract the number of $B$ mesons, we need to account for these structures. Using Monte Carlo simulation, we study differences in trigger and reconstruction efficiencies between $B^{0}$ and $B_{s}^{0}$ mesons. To trust the results of our study, we validate the Monte Carlo simulation by comparing distributions in data to those in Monte Carlo. Finally, we correct the measured rate of reconstructed $B$ mesons by the ratio of efficiencies in order to extract the ratio of branching fractions.

\subsection{Monte Carlo Simulation}

Monte Carlo simulation has been used to study the behavior of physics events in the detectors for a long time. The complexity of detectors and numerous effects which have to be taken into account make analytical derivations of relevant distributions impractical or impossible. Simulation using Monte Carlo techniques is especially useful when studying physics events which have not been registered by the detector for some reason, or events whose origin is difficult to determine. We use the Monte 
Carlo simulation for these two purposes in our analysis.

The first application is to model the fitting function where it is crucial to understand the exact composition of the candidates we reconstruct. The second application is the determination of trigger and reconstruction efficiencies.

For our normalization sample, we choose a kinematically similar mode with large statistics, $B^{0} \rightarrow D^{-} \pi^{+}$. For our measurement, we will need to extract the ratio of efficiencies for the two modes. Therefore, our result does not require precise knowledge of the absolute values of trigger and reconstruction efficiencies, because these effects cancel to first order in the ratio. The differences between the two decays are discussed in the section dealing with systematic uncertainties.

Before going into any detail on how the Monte Carlo was used, it is crucial that we understand what the Monte Carlo simulation is based on. There are several components in the simulation. Only if we understand each component we can evaluate in how far the simulation can be trusted. The components are:

- $B$ meson production and decay

- quark production mechanism

- fragmentation

$-B$ meson decay

- description of the trigger

- description of the reconstructed quantities

The first three components are theory related while the last two are related to the simulation of the detector.

\subsubsection{B Hadron Generation and Decay}

Because we have an exclusive reconstruction and a clean signal, we can confine ourselves to simulating only the $B$ meson which makes our candidate. By applying sideband subtraction, any quantity of the particular $B$ meson decay is studied and 
compared to the Monte Carlo simulation. Therefore, the production mechanism and fragmentation process are not relevant to the measurement as long as the correct momentum spectrum and angular distribution are produced. The decay characteristics are well modeled by the commonly used decay programs. We use Bgenerator [48] as the Monte Carlo program to generate $B$ mesons. It is based on NLO calculations [49] and the fragmentation is implemented using the Peterson fragmentation function [50]. Although recently more up-to-date fragmentation models have become available [51] it is not critical to this analysis since we can modify the $p_{T}$ and $\eta$ dependencies to correct for imperfect description of the underlying processes. Bgenerator only produces $B$ mesons, no fragmentation products or proton remnants are present. For simulating the $B$ meson decays we use the EvtGen program [52]. This program has been extensively tuned by the experiments at the $\Upsilon(4 S)$ resonance. The $B^{+}$and $B^{0}$ components are very well understood. Much less so the $B_{s}^{0}$ and the $b$ baryon decays.

\subsubsection{Realistic Simulation}

The detector simulation is more complicated and is subdivided into the trigger and the reconstructed quantities. The detector geometry and the behavior of its active components is simulated using the GEANT [34] simulation framework. This allows for modeling the detector response at the hit level. This means that the energy deposition in every active and passive component of the detector is simulated. Furthermore, the data acquisition systems response to the active detector components is also simulated and the output of the simulation mimics the real data structure. This way the data reconstruction program can be directly applied to outputs of the simulation.

In its first incarnation, the simulation assumed that the detector functions perfectly which means that all components have the proper high voltage and the electronics react to traversing particles as designed. Real data also includes time dependent inefficiencies, electronic noise and effects from malfunctioning subdetector parts. For instance, fractions of the silicon detector have been turned off temporarily or even for good, and the active coverage of the silicon system directly affects the efficiency of the SVT-based triggers. The parameters of the trigger subsystems have also changed 
on various occasions. The positioning of the beams with respect to the detector and the positioning of the silicon is only known to a certain extent and has to be taken into account when performing simulations.

To account for these imperfections in the detector simulation, a special realistic Monte Carlo simulation has been developed [53]. The data taking period is divided into sub-periods where the detector performance is constant. This includes the parameters of the triggers at Level 1 and Level 2, the position of the beamline, the silicon detector conditions and the alignment. The COT is assumed to have constant behavior.

Because there are occasional glitches in various detector subsystems which are not described in this simulation, only runs where conditions are known to be reproducible are selected. More information about the selection of good runs can be found in Section 4.1.

We generate the Monte Carlo for both the winter (hbot0h) and summer (hbot1i) data using the latest available version of the realistic simulation package. The generation of the events is done with Bgenerator [48], using the NDE spectrum for single $b$ quarks. The $b$ quark mass is set to $4.75 \mathrm{GeV} / \mathrm{c}^{2}$, and the Peterson fragmentation parameter is set to $\epsilon_{B}=0.006$. The minimum $b$ quark $p_{T}$ is set to $0 \mathrm{GeV} / \mathrm{c}$, and the maximum absolute pseudorapidity of the $b$ quark is set to $|\eta|<10$. The generation, decay and GEANT simulation of the detector response are done using the 4.11.1 version of cdfSim. The response of the trigger simulation is done using the TRGSim++ executable as available with the 4.9.1hpt3 release of CDF-II software. The production executable used is the ProductionExe as available from the 4.9.1hpt3 release. The trigger decision is simulated using the SvtFilter executable of the realistic trigger simulation package. This executable confirms cuts equivalent to those for the B_CHARM trigger path at L2 on the SVTD_StorableBank information available in the event record. 


\section{$5.2 \quad$ Fitting $B$ Mass Spectra}

The mass spectra for all three $B$ mesons have interesting features which are already visible when doing the analysis with unoptimized cuts. When the optimized cuts are applied, the structures get even more pronounced. Figure 4-6 shows a significant structure below the main signal peak for every flavor of $B$ meson investigated. We use Monte Carlo simulated events to study the origin of the structures seen in the data.

We generate single $b$ hadrons using BGenerator and decay them using EvtGen. We generate three different Monte Carlo samples: generic $B \rightarrow D_{s}^{-} X$, where the $D_{s}^{-}$decay is forced: $D_{s}^{-} \rightarrow \phi \pi^{-}\left(\phi \rightarrow K^{+} K^{-}\right)$, generic $B \rightarrow \overline{D^{0}} \pi$, where the $D^{0}$ decay is forced: $\overline{D^{0}} \rightarrow K^{-} \pi^{+}$and generic $B \rightarrow D^{-} X$, where the $D^{-}$decay is forced: $D^{-} \rightarrow K^{+} \pi^{-} \pi^{-}$. "Generic $b$ " decays means a mixture of $B^{+}, B^{0}, B_{s}^{0}$ and $b$ baryons is generated with fractions according to the world average values [10]. The events are then filtered and reconstructed using the realistic simulation framework, and analyzed with the same reconstruction software and selection requirements that are used to reconstruct the different $B$ mesons. The resulting mass spectra are decomposed based on the recorded Monte Carlo decay history information, so that contributions from different decay modes are isolated. The spectra with different contributions are shown in Figures5-1, 5-3 and 5-5 for $B^{0}, B^{+}$and $B_{s}^{0}$.

All the $B$ backgrounds have common features: the Cabibbo suppressed decays produce a small contamination of the main signal peak and there is a structure just below it. The rest of the background is continuous, and it turns on at low enough $B$ masses that it does not pollute the main $B$ peak. In the case of the $B^{+}$mesons, the structure below the main $B$ peak has two pronounced "spikes". The mechanism that produces this structure is the result of a $B \rightarrow D^{*} \pi$ decay followed by a $D^{*} \rightarrow D^{0} \pi$ decay. The world average values [10] of branching fractions for $D^{*} \rightarrow D \pi$ decays are given in Table 5.2.

The $D^{*}$ is completely polarized in this decay. As a result, the angle between the momentum of the soft pion in the $D^{*}$ decay and the $D^{*}$ flight direction follows a 


\begin{tabular}{l|c} 
Decay mode & Branching Fraction \\
\hline \hline$D^{* 0} \rightarrow D^{0} \pi^{0}$ & $61.9 \pm 2.9 \%$ \\
$D^{*+} \rightarrow D^{0} \pi^{+}$ & $67.7 \pm 0.5 \%$ \\
$D^{*+} \rightarrow D^{+} \pi^{0}$ & $30.7 \pm 0.5 \%$ \\
\hline$D^{* 0} \rightarrow D^{0} \gamma$ & $38.1 \pm 2.9 \%$ \\
$D^{*+} \rightarrow D^{+} \gamma$ & $1.6 \pm 0.4 \%$ \\
$D_{s}^{*+} \rightarrow D_{s}^{+} \gamma$ & $94.2 \pm 2.5 \%$
\end{tabular}

Table 5.1: Branching fractions for various $D^{*}$ decays

$\cos ^{2}(\theta)$ distribution. Most of the time, the pion is released either in the same direction as the $D^{*}$ momentum, or in the opposite direction. Depending whether the pion was released in the same or opposite direction to the $D^{*}$ flight direction, the missing mass is quantized. Either most of the free energy of the decay will be carried away by the soft pion or very little. The $Q$ value of the $D^{*}$ decay is small $\left(\sim 6 \mathrm{MeV} / \mathrm{c}^{2}\right)$, and the polarization effect causes the values of the $B$ mass to pile up in either of the two spikes.

The two "spikes" are very pronounced for $B^{+}$decays, because both $B^{0} \rightarrow D^{*-} \pi^{+}$ and $B^{+} \rightarrow \overline{D^{* 0}} \pi^{+}$decays contribute, because a large fraction of both $D^{*-}$ and $D^{* 0}$ decay to a $D^{0}$ meson and a pion. The "spikes" are less pronounced for $B^{0}$ decays, because only $B^{0} \rightarrow D^{*-} \pi^{+}$decays contribute to this mass spectrum. In the case of $B_{s}^{0}$ decays, we do not expect to see a very prominent structure, because in the analogous $B_{s}^{0} \rightarrow D_{s}^{*-} \pi^{+}$, the $D_{s}^{*-}$ decays to $D_{s}^{-}$and a photon $94 \pm 2.5 \%$ of the time [10], so the decay mechanism is different and this determines the shape of the background structure.

The mass spectra after applying optimized cuts are shown in Figure4-6. To extract the number of signal events a fit of the lineshapes is applied. The number of signal events is determined by integrating the function describing the signal. The following subsections give a complete, detailed description of the fit and motivate the various components. 

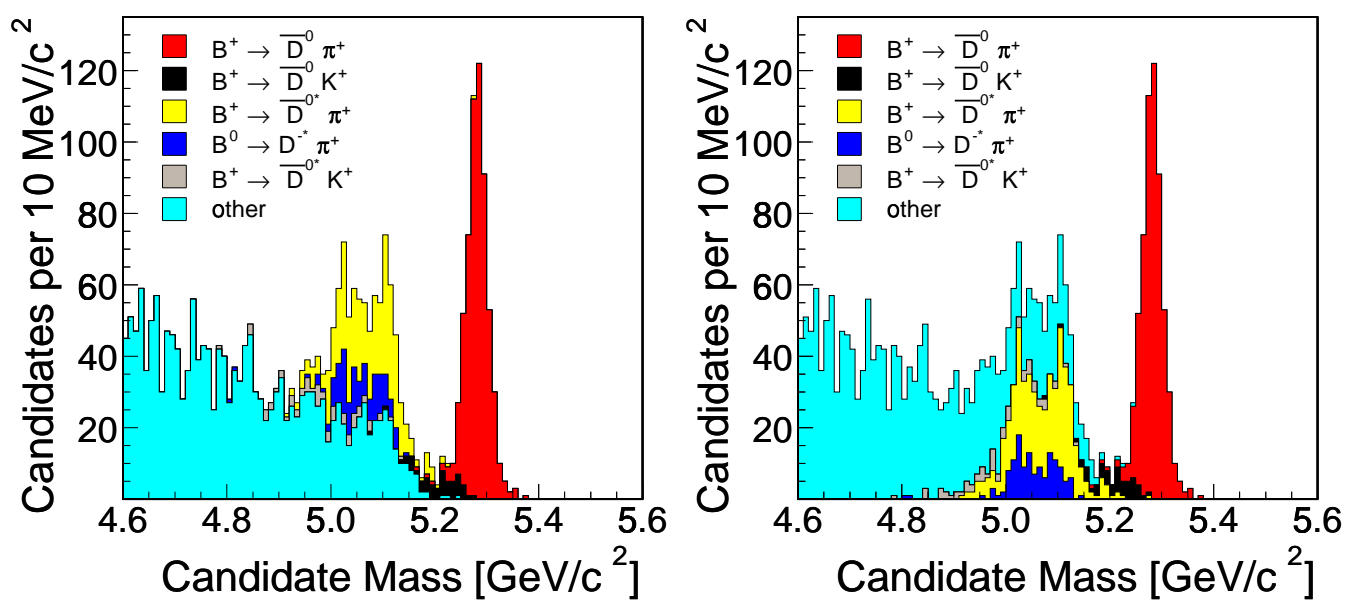

Figure 5-1: Composition of the single- $B$ background for $B^{+}$decays. In the left plot, the decomposition is done in such a way that it shows which decays cause the spiked structure in the mass peak. In the right plot, the different contributions are stacked to show the shapes of the different decay contributions.
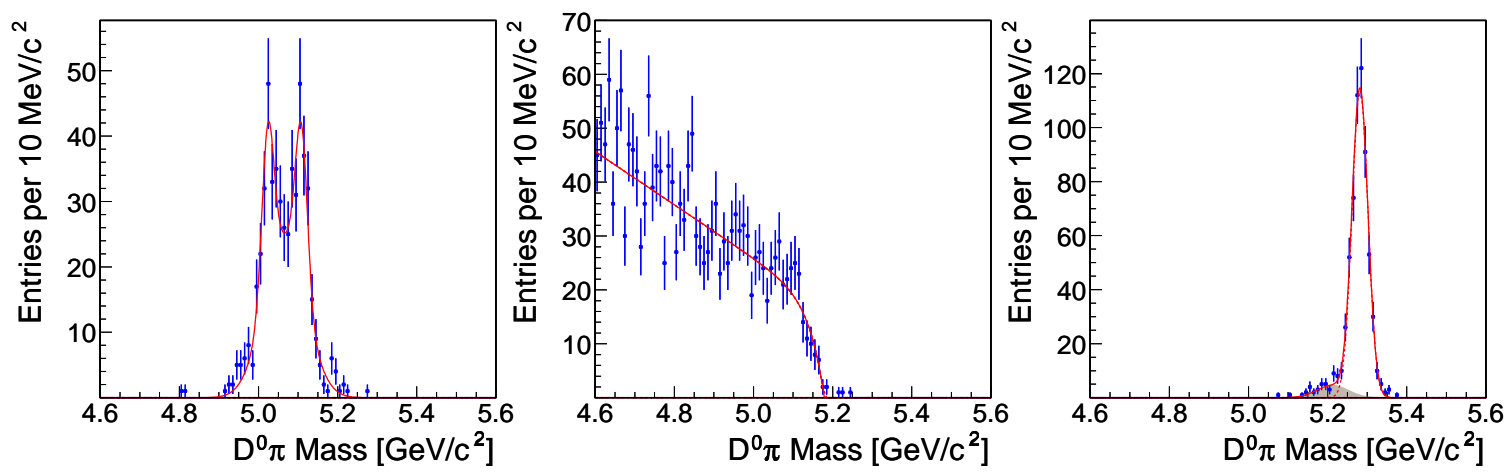

Figure 5-2: Templates used in $B^{+} \rightarrow D^{0} \pi$ fitting: on the left, $B \rightarrow D^{*} \pi$ decays where the pion from the $D^{*}$ decay is not reconstructed. The polarization of the $D^{*}$ causes the double peak structure. The center plot depicts the smooth continuum distribution, and the right plot shows the signal distribution with the Cabibbo suppressed $D^{0} K$ decay in the shaded region. The analytical templates used to fit data are overlaid. 

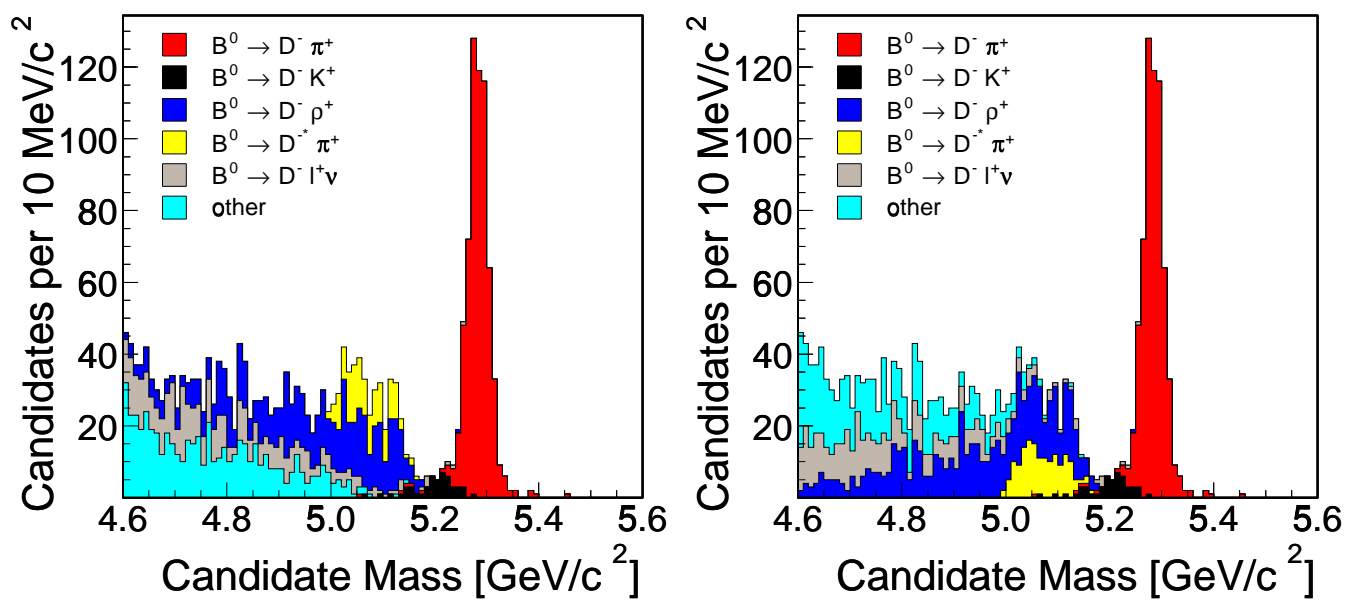

Figure 5-3: Composition of the single- $B$ background for $B^{0}$ decays. In the left plot, the decomposition is done so that it shows which decays cause the spiked structure in the mass peak. In the right plot, the different contributions are stacked to show the shapes of the different decay contributions.
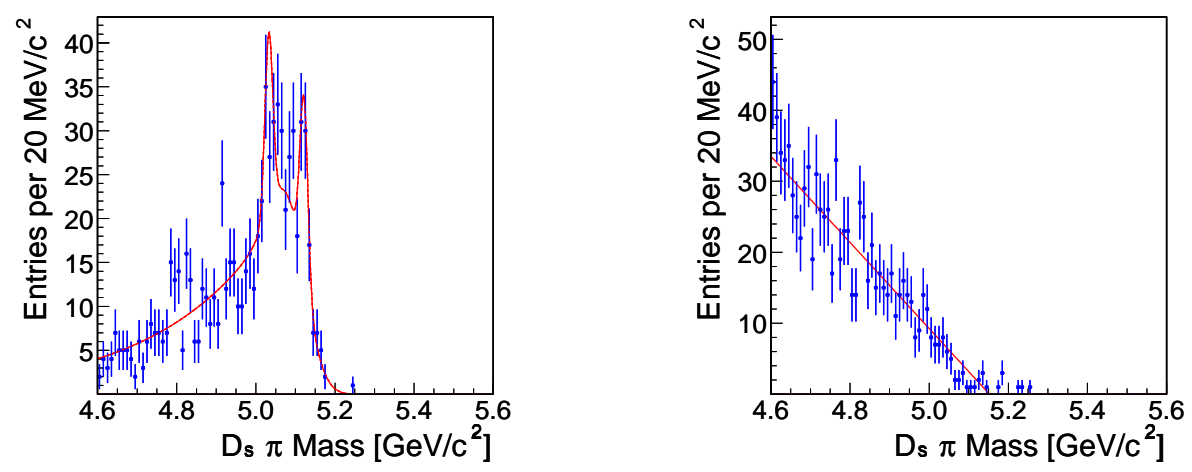

Figure 5-4: Templates used in $B^{0} \rightarrow D^{+} \pi$ fitting. On the left, the structure due to $D^{*} \pi$ and $D_{s} \rho^{+}$decays, with the double peak structure caused by $D^{*}$ decays. The right plot shows the smooth distribution from other $D_{s} X$ decays. The analytical templates used to fit the data are overlaid. 

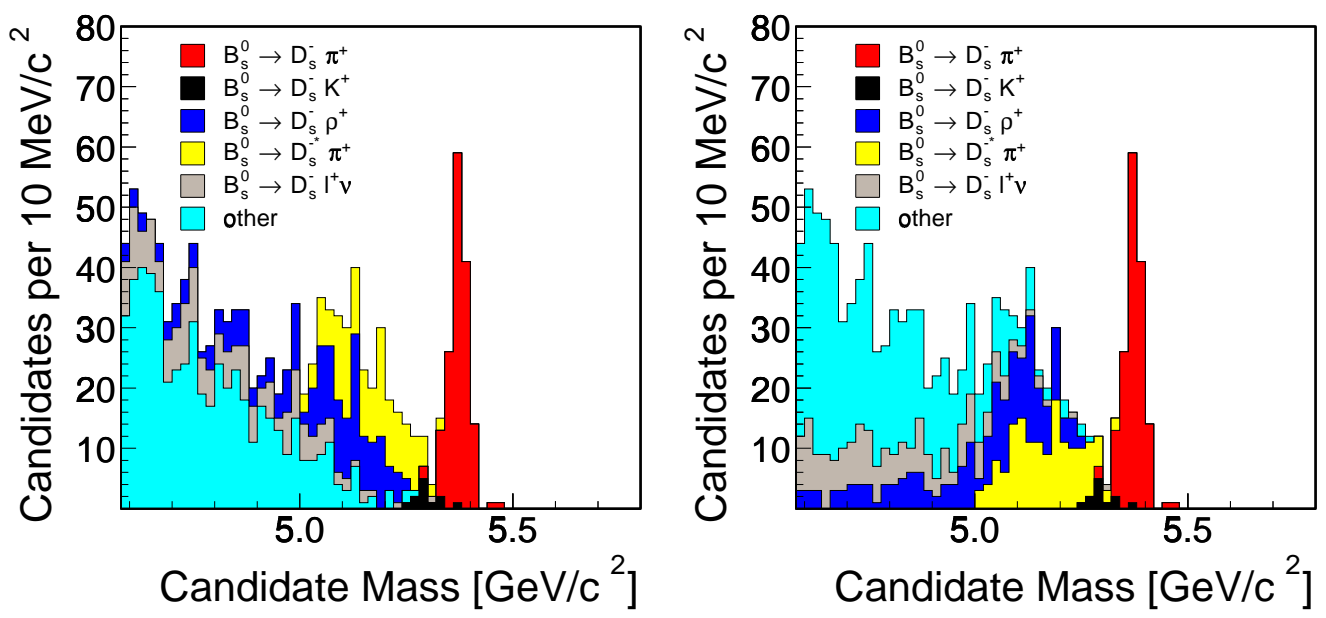

Figure 5-5: Composition of the single- $B$ background for $B_{s}^{0}$ decays. In the left plot, the decomposition is done so that it shows which decays cause the spiked structure in the mass peak. In the right plot, the different contributions are stacked to show the shapes of the different decay contributions.
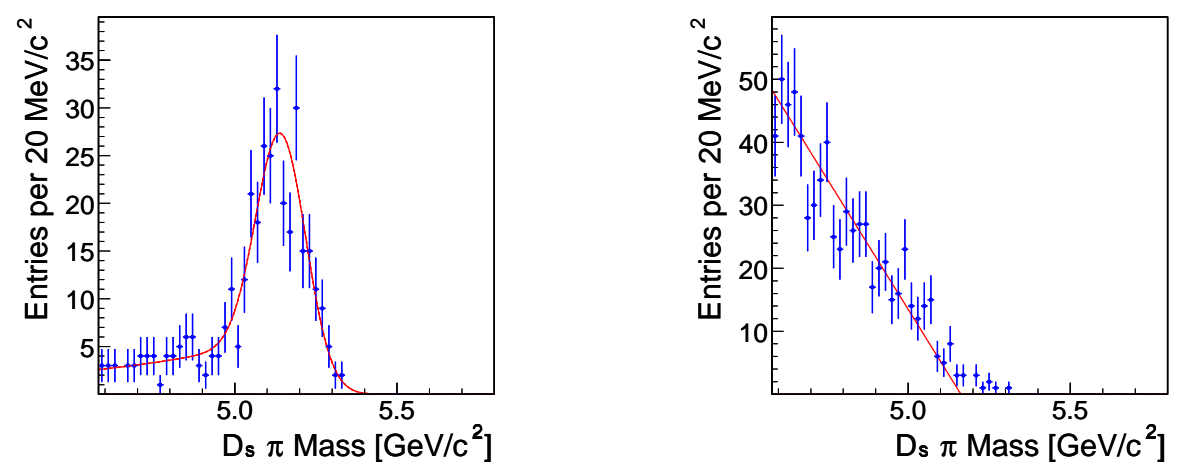

Figure 5-6: Templates used in $B_{s} \rightarrow D_{s} \pi$ fitting: left, the structure due to $D_{s}^{*} \pi$ and $D_{s} \rho^{+}$decays. No double peak structure is seen because the kinematics of $D_{s}^{*+} \rightarrow D_{s}^{+} \gamma$ do not produce such structures. The right plot shows the smooth distribution from other $D_{s} X$ decays. The analytical templates used to fit the data are overlaid. 


\subsubsection{General Fit Model}

All reconstructed mass spectra have quite interesting structures. There is a main peak around the mass of the $B$ meson in question which constitutes the signal. Its width is driven by the detector resolution and is therefore described by a Gaussian distribution. It could be argued that several Gaussians are more appropriate but we decide to use only one Gaussian because the data are reasonably well described. There is a rich structure of reflections in the lower mass regions which is most pronounced in the $B^{+}$case. The structure of those reflections is usually due to kinematically similar decays where a pion is not reconstructed. In particular soft pions from $D^{*}$ decays have a significant structure due to the low $Q$ value of the reaction. It is essential to describe those reflections well to properly subtract them when they leak into the signal area. The following general strategy is applied to all modes. The details for each particular fit is discussed in the following subsections.

Monte Carlo studies are performed in which large numbers of inclusive decays are generated. Particular decays which are prone to produce a spiky reflection structure are singled out and grouped into one component which is then templated. This means that a function is developed to describe the shape as precisely as possible. Parts of that function are motivated by physics arguments and other parts are just terms which fit the distributions best.

The Cabibbo suppressed decays, $B \rightarrow D K$, are modeled as simple Gaussians. The central position and the width is taken from the Monte Carlo. The normalization is fixed to the PDG ratio [10] with respect to the signal peak. The $B \rightarrow D K$ decays appear in our mass spectrum because we use no particle identification and therefore cannot distinguish this decay from the $B \rightarrow D \pi$ decay. This effect is known as a reflection peak. Reflection peaks often tend to be distorted and non-Gaussian. For this particular reflection and the statistics relevant to our sample, we find that modeling it with a Gaussian distribution is sufficient.

The remaining Monte Carlo contribution which has no significant structure, is typically modeled by a linear functions or exponential. This takes care of the con- 
tributions from the remaining partially reconstructed $B$ decays. Combinatorics are modeled by a simple exponential which takes over in the upper mass region beyond the signal peak. For the fit the contributions from $B$ decays, three templates (spiky, Cabibbo suppressed and smooth), are mostly fixed to the parameters obtained from fits to the Monte Carlo. All the parameters of the the signal Gaussian and the exponential for the combinatorics are let to float freely.

\subsubsection{Fit Model: Signal and Cabibbo Suppressed Decays}

Our approach to modeling the signal is same in all three cases, so we deal with its implementation first. In all cases, the signal peak and the reflection from the Cabibbo suppressed $B \rightarrow D K$ decay are very close in the mass spectrum, so they can be modeled with one template.

We chose to model the signal peak with a single Gaussian distribution. One could argue that multiple Gaussian distributions would be more appropriate because of the different classes of quality of reconstructed $B$ mesons. We find that fits using only one Gaussian distribution describes the data well. At the level of statistics of our measurement, our Monte Carlo studies show that the Cabibbo suppressed $B \rightarrow D K$ decay is also well described with a single Gaussian distribution.

Therefore, the signal template is composed of two Gaussians; one for the signal peak and one for the Cabibbo suppressed reflection. The fit function is:

$$
f_{S}(m)=N_{S} \cdot\left[\mathcal{G}(m \mid \mu, \sigma)+f_{C} \cdot \mathcal{G}\left(m \mid \mu-\delta_{C}, \sigma_{C}\right)\right]
$$

where $N_{S}$ is the overall normalization, $\mu$ and $\sigma$ are the parameters for the signal Gaussian, $f_{C}$ is the scale factor for the Cabibbo suppressed Gaussian, $\delta$ is the offset of the mean of the Cabibbo Gaussian, and $\sigma_{C}$ is its width. From the fits to our Monte Carlo sample, we derive the offset between the Cabibbo suppressed peak and the main signal peak. $\delta$, and the ratio of Cabibbo suppressed to signal events, $f_{C}$. These parameters are then kept constant in the fit to the data. We trust our Monte Carlo to reproduce relative numbers (relative yield of one $B$ meson to another, the mass 
difference between the signal peak and the Cabibbo reflection) better than absolute numbers (absolute values of the means of the Gaussians). The values of the fixed parameters are extracted separately for each of the three $B$ mesons modeled.The remaining parameters, $N_{S}, \mu$ and $\sigma$ are allowed to float in the fit.

\subsubsection{Fit Model: $B^{+}$}

The $B^{+}$decay has the most prominent reflection structure which is mostly due to the $B^{+} \rightarrow D^{* 0} \pi^{+}$which has a larger branching fraction than the corresponding modes in $B^{0}$ or $B_{s}$. There are also contributions from $B^{0} \rightarrow D^{*+} \pi^{+}$which have no equivalent in the other decays.

The reflection has a peculiar two horn structure which is caused by the polarization of the $D^{*}$ in the decay. To make up the spiky template the $B^{+} \rightarrow D^{* 0} \pi^{+}$and $B^{0} \rightarrow D^{*-} \pi^{+}$spectra are combined. The combined mass spectrum is then modeled with a combination of three Gaussian distributions. One models the body of the structure, and the other two are placed symmetrically around it to model the two spikes:

$$
f_{H}(m)=N_{H} \cdot\left[(1-f) \cdot \mathcal{G}\left(m \mid \mu, \sigma_{B}\right)+f \cdot\left[0.5 \cdot \mathcal{G}\left(m \mid \mu-\delta, \sigma_{H}\right)+0.5 \cdot \mathcal{G}\left(m \mid \mu+\delta, \sigma_{H}\right)\right]\right]
$$

where $N_{H}$ is the normalization, $\mu$ is the mean of the main Gaussian, $\delta$ is the spike half-distance, $\sigma_{B}$ is the width of the main Gaussian, and $\sigma_{H}$ is the width of the spike Gaussians. In the final fit to the data, most of the parameters of the above equation are fixed from Monte Carlo values. The values which are left floating are $N$, which defines the overall normalization, and $\mu$ and $\delta$ which adjust for the slight differences in absolute mass scale between the data and Monte Carlo.

The background contribution from other, unspecified $B \rightarrow D^{0} X$ decays can be simply modeled with a linear function multiplied with an exponential decay function. Our choice of fit function is:

$$
f_{C}(m)=N_{C} \cdot(1.0-(m-4.9) \cdot s) \cdot\left(1-e^{(m-\mu) \cdot \tau)}\right)
$$


where $N$ defines the normalization, $s$ defines the slope, $\mu$ is the turnoff point for our function; we do not define the function beyond this point. $\tau$ is the parameter of exponential decay of of our function toward the turnoff point. The mass spectra for the relevant templates are depicted in Figure 5-2. The analytical templates with the parameters used in the data fits are overlaid.

For our fits to the data, we fix all the parameters in the above equations to the values from Monte Carlo, except for the normalization constants, $N_{C}$ and $N_{H}$. For these two variables, we require that the ratio $N_{C} / N_{H}$ be fixed. The values of parameters extracted from Monte Carlo templates are listed in Table 5.2.

\subsubsection{Fit Model: $\boldsymbol{B}_{s}$}

In the case of $B_{s}$ decays, we do not expect to see a spiky satellite structure as we do for the other two $B$ mesons. The reason for this is the fact that the main contribution to such a structure would be coming from the $B_{s} \rightarrow D_{s}^{*} \pi$ decays, but in this case the $D_{s}^{*}$ decays to $D_{s}$ and a photon most of the time, so the helicity structure is lost. The two decay modes that contribute to the peak below the $B_{s}$ mass are the $B_{s} \rightarrow D_{s}^{*} \pi$ and the $B_{s} \rightarrow D_{s}^{-} \rho^{+}, \rho^{+} \rightarrow \pi^{+} \pi^{0}$ decays. We create one template to model this contribution, and another to model the contribution of all other $B \rightarrow D_{s} X$ backgrounds. We find that the combined distribution for $D_{s}^{*} \pi$ and $D_{s}^{-} \rho^{+}$decays can be modeled with a lifetime distribution and a Gaussian:

$$
f_{R}(m)=N_{R} \cdot[(1-f) \cdot \exp (m \mid \lambda) \otimes \mathcal{G}(m \mid \mu, \sigma)+f \cdot \mathcal{G}(m \mid \mu, \sigma)]
$$

where $N_{R}$ is the normalization, $f$ is the fraction of the events in the Gaussian peak, $\sigma$ is the width of the Gaussian and at the same time the resolution of the lifetime distribution, and $\lambda$ is the decay constant of the low-mass tail. In the fit to the data, all parameters were fixed to Monte Carlo values except for the normalization, $N_{R}$.

We find that the continuum background from all other $D_{s} X$ decays can be modeled 
with a simple linear function:

$$
f_{L}(m)=N_{L} \cdot \frac{2}{(\gamma-L)^{2}} \cdot(\gamma-m)
$$

where $N_{L}$ is the normalization, $\gamma$ is the cutoff point at which the linear function hits zero, and $L$ is the lower boundary of the fitting region.

In the fit to the data, the parameter $\gamma$ is fixed. For the combined function $f_{R}+f_{L}$, we require that the ratios of the normalizations $N_{R} / N_{L}$ be fixed to a value determined from the Monte Carlo. The mass spectra for the different contributions and the corresponding analytical templates are shown in Figure 5-6. The values of parameters extracted from Monte Carlo templates are listed in Table 5.3.

\subsubsection{Fit Model: $B^{0}$}

The $B^{0}$ decay has a much less pronounced reflection than the $B^{+}$but still has very similar features, namely the two spikes are present. The spiky structure here mostly comes from the $B^{0} \rightarrow D^{*-} \pi^{+}$and the $B^{0} \rightarrow D^{-} \rho^{+}$decays. To make up the spiky template $B^{0} \rightarrow D^{*-} \pi^{+}$and $B^{0} \rightarrow D^{-} \rho^{+}$are combined. In this case, we use a similar function to what we had in the $B_{s}$, but we add the spiky contribution with two Gaussians on top:

$$
\begin{array}{r}
f_{R}(m)=N_{R} \cdot\left[\left(1-f_{G}-f_{H}\right) \cdot \exp (m \mid \lambda) \otimes \mathcal{G}\left(m \mid \mu, \sigma_{R}\right)+f_{G} \cdot \mathcal{G}\left(m \mid \mu-\nu, \sigma_{R}\right)+(5 .\right. \\
\left.f_{H} \cdot\left(0.5 \cdot \mathcal{G}\left(m \mid \mu-\nu-\delta, \sigma_{H}\right)+0.5 \cdot \mathcal{G}\left(m \mid \mu-\nu+\delta, \sigma_{H}\right)\right)\right]
\end{array}
$$

where $N_{R}$ is the signal normalization, $f_{G}$ and $f_{H}$ are the fractions in the main Gaussian and the spikes respectively, $\mu$ is the zero-point of the lifetime distribution, $\nu$ is the offset of the mean of the main Gaussian, and $\delta$ is the half-distance between the horns. The relevant widths are $\sigma_{R}$ both for the main Gaussian and the resolution of the lifetime convolution and $\sigma_{H}$ for the spikes. All these parameters are fixed from fits to the Monte Carlo, except for the normalization constant $N_{R}$. The continuum background is modeled with the same parametrization of the linear function $\left(f_{L}\right)$ as 
is used for the $B_{s}$, but the values of the parameters are different.

In the overall fit to the data, we require that the ratio of normalization constants $N_{R} / N_{L}$ be fixed to the value extracted from the Monte Carlo, because these depend on the relative branching fractions which are assumed to be correct in the Monte Carlo simulation. Again, to allow for small discrepancies on the absolute mass scale between the data and Monte Carlo, we allow the offsets $\nu$ and $\delta$ to float in the fit. The mass spectra for the different contributions and the corresponding analytical templates are shown in Figure 5-4. The values of parameters extracted from Monte Carlo templates are listed in Table 5.4.

\subsection{6 $\quad B$ Meson Yields}

We reconstruct $B$ mesons using optimized selection requirements described in Section 4.3.5 and apply fits using analytical templates described in Section 5.2.1 to the spectra. The resulting fits are shown in Figures 5-7, 5-8 and 5-9. The number of candidates extracted from the fits are listed in Tables 5.5, 5.7 and 5.6. The errors quoted in the tables are statistical only. To determine the systematic error on the number of candidates, an independent study is performed.

The yields and mass spectra are first broken down between the winter and summer datasets; we expect the absolute rates in these two samples to be slightly different because the trigger was more efficient in the summer. The $B_{s}^{0}$ signal, shown in Figure 5-8 is cleaner than the $B^{+}$and $B^{0}$ signals, shown in Figures 5-7 and 5-9, respectively. This comes from requiring that the reconstructed invariant mass of the $\phi \rightarrow K^{+} K^{-}$ be within a tight window around the world average value, as described in Section 4.3.5. The $B^{+}$and $B^{0}$ decays do not have equivalent requirements. 


\begin{tabular}{c|l|c} 
Parameter & Meaning & Value \\
\hline \hline$f_{C}$ & Cabbibo supp. fraction & $0.079 \pm 0.013$ \\
$\delta_{C}$ & Cabbibo mass diff $\left[\mathrm{GeV} / \mathrm{c}^{2}\right]$ & $0.069 \pm 0.011$ \\
$\sigma_{C}$ & Cabbibo supp. width $\left[\mathrm{GeV} / \mathrm{c}^{2}\right]$ & $0.040 \pm 0.009$ \\
$f$ & spike fraction & $0.368 \pm 0.137$ \\
$\sigma_{B}$ & main body width $\left[\mathrm{GeV} / \mathrm{c}^{2}\right]$ & $0.052 \pm 0.005$ \\
$\sigma_{H}$ & spike width $\left[\mathrm{GeV} / \mathrm{c}^{2}\right]$ & $0.015 \pm 0.004$ \\
$N_{C} / N_{H}$ & continuum fraction & $0.768 \pm 0.013$ \\
$s$ & slope $\left[1 / \mathrm{GeV} / \mathrm{c}^{2}\right]$ & $1.601 \pm 0.217$ \\
$\tau$ & turnoff lifetime $\left[1 / \mathrm{GeV} / \mathrm{c}^{2}\right]$ & $29.978 \pm 9.162$
\end{tabular}

Table 5.2: Shape-defining template parameters and errors extracted from $D^{0} X$ generic Monte Carlo, used in fitting for the structures in the $B^{+} \rightarrow D^{0} \pi$ mass spectrum. In the fit to the data, these parameters were set to the mean values and kept constant.

\begin{tabular}{c|l|c} 
Parameter & Meaning & Value \\
\hline \hline$f_{C}$ & Cabbibo suppressed fraction & $0.079 \pm 0.023$ \\
$\delta_{C}$ & Cabbibo mass diff $\left[\mathrm{GeV} / \mathrm{c}^{2}\right]$ & $0.075 \pm 0.009$ \\
$\sigma_{C}$ & Cabbibo supp. width $\left[\mathrm{GeV} / \mathrm{c}^{2}\right]$ & $0.031 \pm 0.006$ \\
$\lambda$ & refl. "lifetime" $\left[1 / \mathrm{GeV} / \mathrm{c}^{2}\right]$ & $0.680 \pm 0.552$ \\
$f$ & reflection gaus. frac & $0.678 \pm 0.061$ \\
$\sigma$ & reflection width $\left[\mathrm{GeV} / \mathrm{c}^{2}\right]$ & $0.077 \pm 0.005$ \\
$N_{R} / N_{L}$ & "B continuum" frac & $0.625 \pm 0.021$ \\
$L$ & "B continuum" cutoff $\left[\mathrm{GeV} / \mathrm{c}^{2}\right]$ & $5.163 \pm 0.113$
\end{tabular}

Table 5.3: Shape-defining template parameters and errors extracted from $D_{s} X$ generic Monte Carlo used in fitting for the structures in the $B_{s}^{0} \rightarrow D_{s}^{-} \pi$ mass spectrum. In the fit to the data, these parameters were set to the mean values and kept constant. 


\begin{tabular}{c|l|c} 
Parameter & Meaning & Value \\
\hline \hline$f_{C}$ & Cabbibo suppressed fraction & $0.067 \pm 0.023$ \\
$\delta_{C}$ & Cabbibo mass diff $\left[\mathrm{GeV} / \mathrm{c}^{2}\right]$ & $0.067 \pm 0.006$ \\
$\sigma_{C}$ & Cabbibo supp. width $\left[\mathrm{GeV} / \mathrm{c}^{2}\right]$ & $0.323 \pm 0.009$ \\
$\lambda$ & reflect. "lifetime" $\left[\mathrm{GeV} / \mathrm{c}^{2}\right]$ & $0.282 \pm 0.038$ \\
$f_{G}$ & reflection Gaus. frac. & $0.065 \pm 0.083$ \\
$\sigma_{R}$ & reflection width $\left[\mathrm{GeV} / \mathrm{c}^{2}\right]$ & $0.040 \pm 0.007$ \\
$f_{H}$ & spike frac & $0.134 \pm 0.037$ \\
$\delta$ & spike separation $\left[\mathrm{GeV} / \mathrm{c}^{2}\right]$ & $0.036 \pm 0.004$ \\
$\sigma_{H}$ & spike width $\left[\mathrm{GeV} / \mathrm{c}^{2}\right]$ & $0.015 \pm 0.004$ \\
$N_{R} / N_{L}$ & continuum frac & $0.568 \pm 0.019$ \\
$\chi$ & continuum cutoff $\left[\mathrm{GeV} / \mathrm{c}^{2}\right]$ & $5.152 \pm 0.006$
\end{tabular}

Table 5.4: Shape-defining template parameters and errors extracted from $D^{+} X$ generic Monte Carlo, used in fitting for the structures in the $B^{0} \rightarrow D^{-} \pi$ mass spectrum. In the fit to the data, these parameters were set to the mean values and kept constant. 

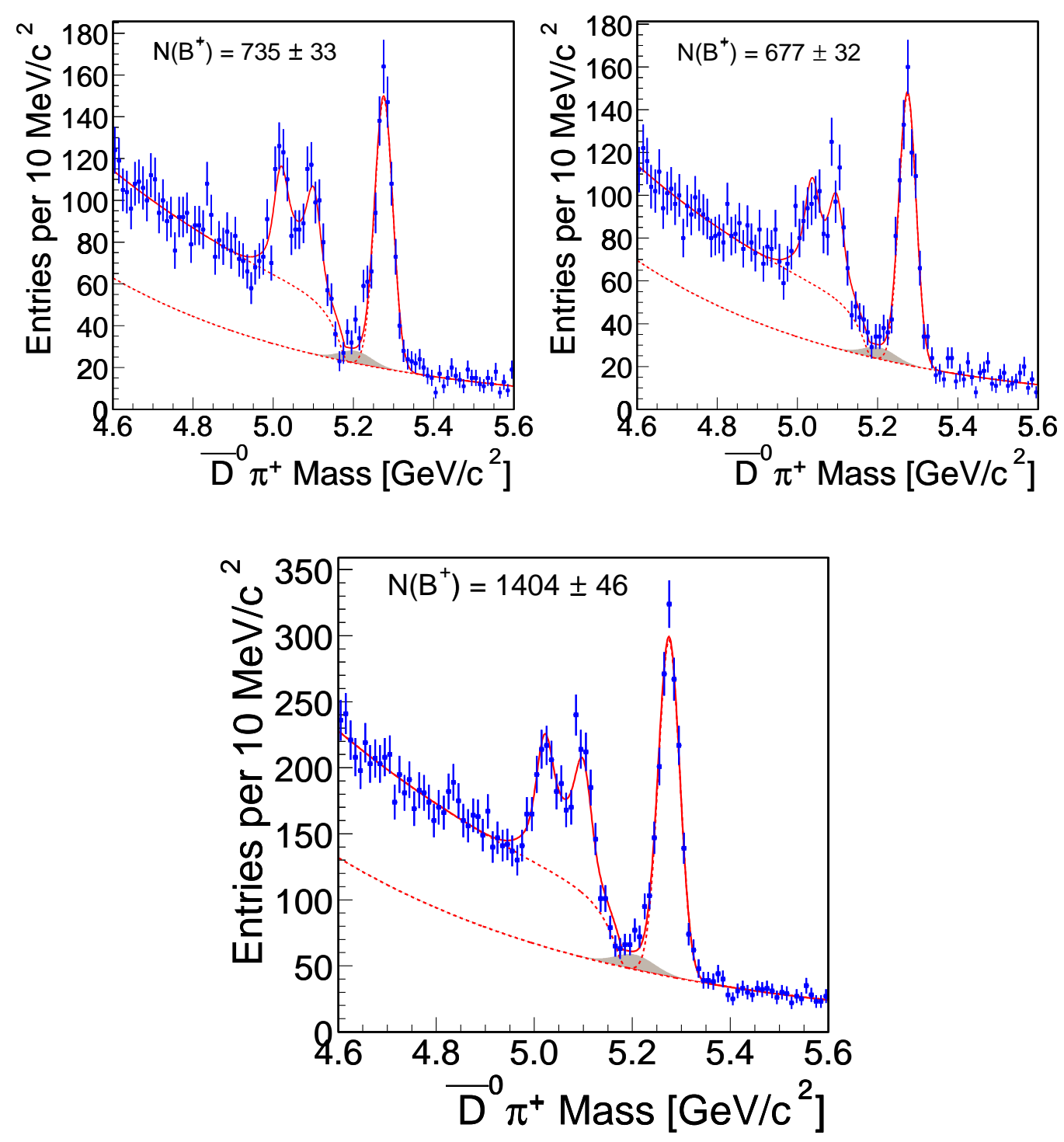

Figure 5-7: Invariant mass distribution for $B^{+} \rightarrow D^{0} \pi^{+}$candidates reconstructed with optimized selection requirements. The left plot shows the distribution for the winter data (hbot0h), and the right plot shows the same distribution for the summer data (hbot1i). The distributions are fitted with an exponential and three Gaussians. In the pre-shutdown data, the number of $B^{+}$candidates is $735 \pm 33$, and in the postshutdown data, this number is $677 \pm 32$. Combining both samples, the fit result yields $1404 \pm 46 B^{+}$. 

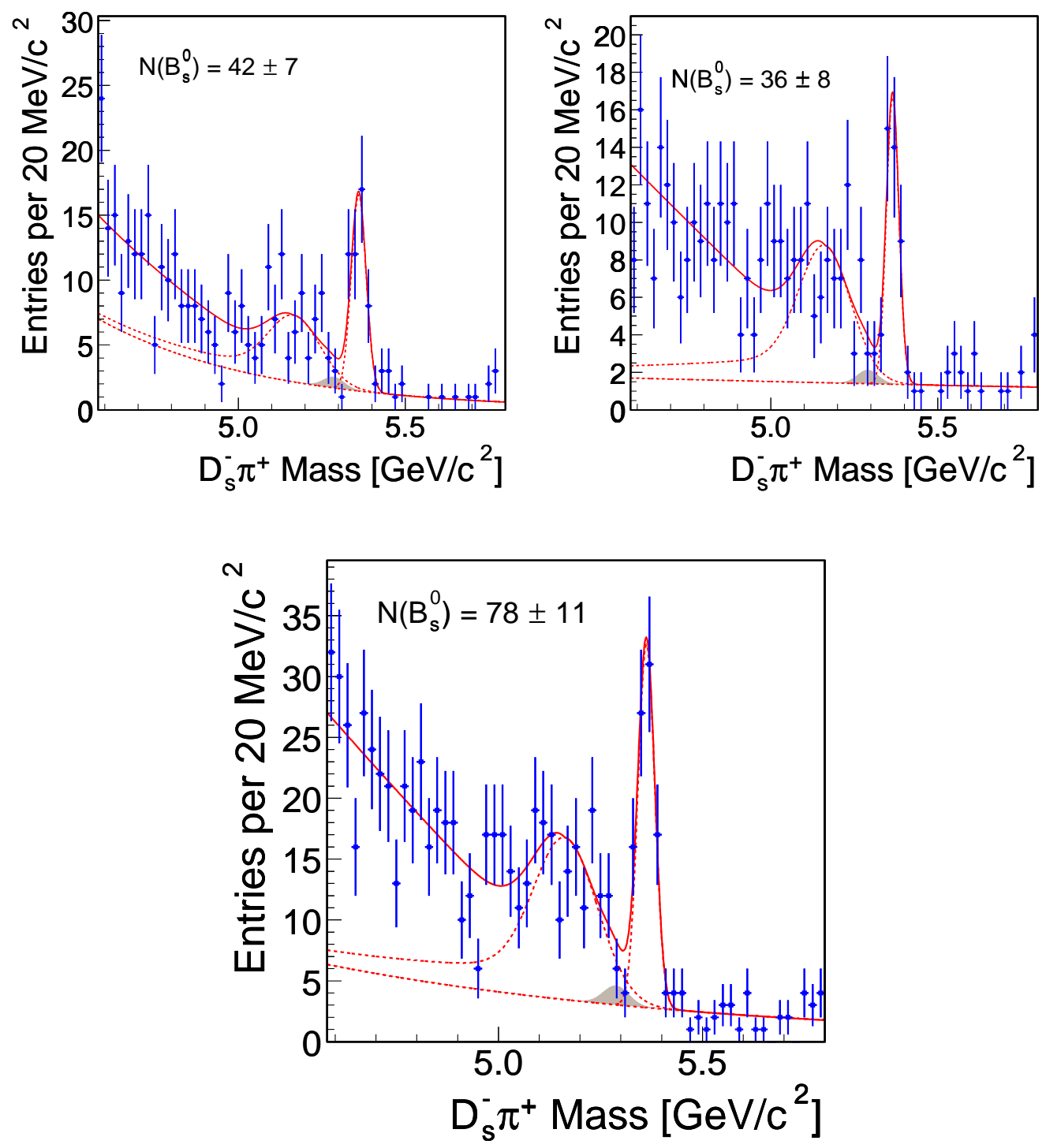

Figure 5-8: Invariant mass distribution for $B_{s}^{0} \rightarrow D_{s} \pi^{+}$candidates reconstructed with optimized selection requirements. The left plot shows the distribution for the winter dataset (hbot $0 \mathrm{~h}$ ), the middle plot shows the same distribution for the summer dataset (hbot1i), and the right plot is the sum of the two distributions. The distributions are fitted with an exponential and two Gaussians. In the pre-shutdown data, the number of $B_{s}^{0}$ candidates is $42 \pm 7$, and in the post-shutdown data, this number is $36 \pm 8$. Combining the two samples, the fit result yields $78 \pm 11$ candidates. 

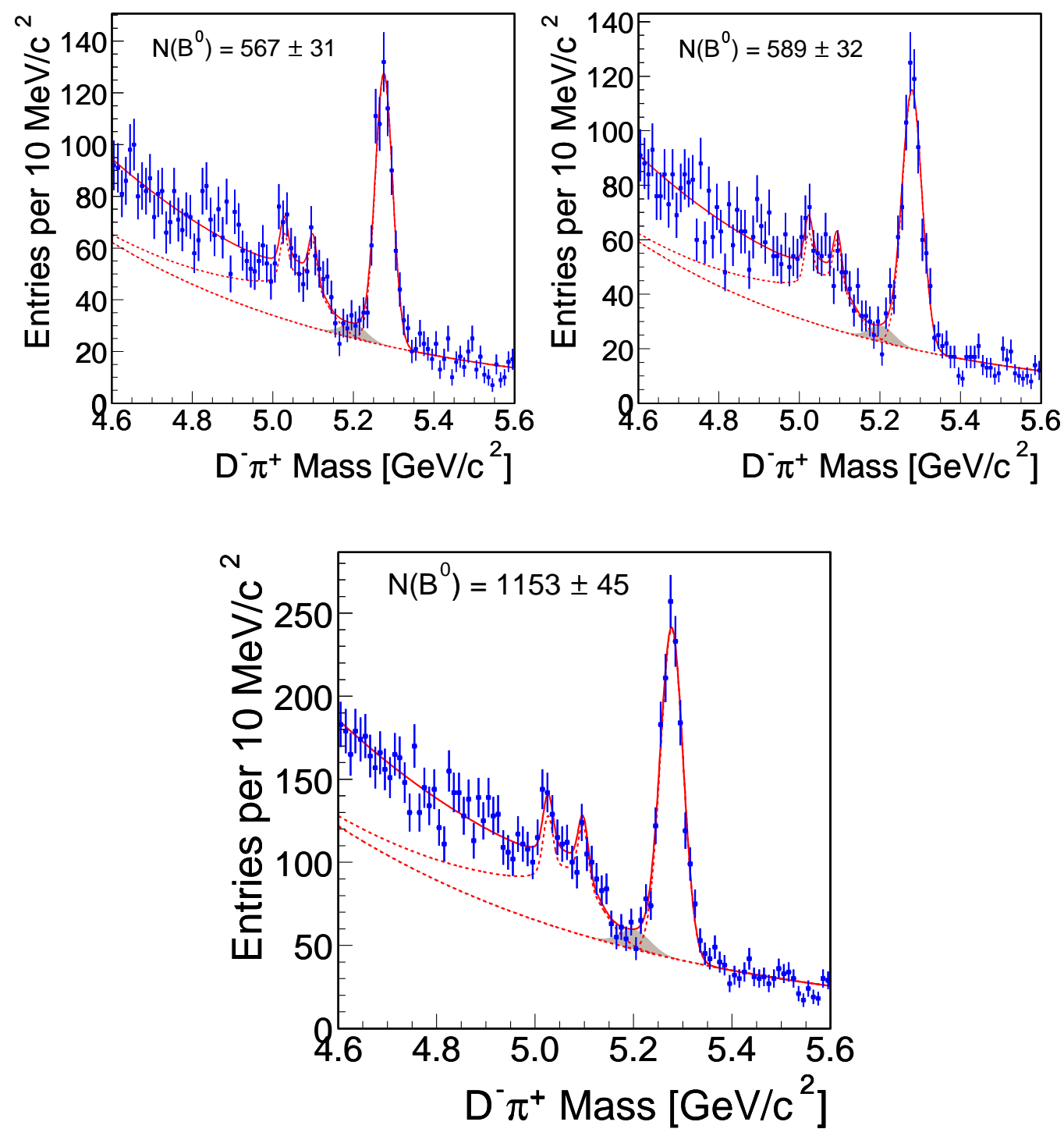

Figure 5-9: Invariant mass distribution for $B^{0} \rightarrow D^{-} \pi^{+}$candidates reconstructed with optimized selection requirements. The left plot shows the distribution for the winter dataset (hbot0h), and the right plot shows the same distribution for the summer dataset (hbot1i). The distributions are fitted with an exponential and three Gaussians. In the pre-shutdown data, the number of $B^{0}$ candidates is $567 \pm 31$, and in the post-shutdown data, this number is $589 \pm 32$. Combining the two samples, the fit result yields $1153 \pm 45$. 


\begin{tabular}{c|c|c|c} 
& hbot0h & hbot1i & combined \\
\hline \hline Events & $735 \pm 33$ & $677 \pm 32$ & $1404 \pm 46$ \\
Mass $\left[\mathrm{MeV} / \mathrm{c}^{2}\right]$ & $5275.5 \pm 1.1$ & $5274.5 \pm 1.1$ & $5274.9 \pm 0.8$ \\
Width $\left[\mathrm{MeV} / \mathrm{c}^{2}\right]$ & $22.7 \pm 1.2$ & $21.3 \pm 1.0$ & $22.0 \pm 0.8$
\end{tabular}

Table 5.5: Fit results for $B^{+} \rightarrow \overline{D^{0}} \pi^{+}$decays in the different datasets. Only statistical errors are quoted.

\begin{tabular}{c|c|c|c} 
& hbot0h & hbot1i & combined \\
\hline \hline Events & $42 \pm 7$ & $36 \pm 8$ & $78 \pm 11$ \\
Mass $\left[\mathrm{MeV} / \mathrm{c}^{2}\right]$ & $5360.6 \pm 4.3$ & $5365.0 \pm 3.9$ & $5362.7 \pm 3.1$ \\
Width $\left[\mathrm{MeV} / \mathrm{c}^{2}\right]$ & $22.1 \pm 3.4$ & $18.7 \pm 3.1$ & $20.7 \pm 2.3$
\end{tabular}

Table 5.6: Fit results for $B_{s}^{0} \rightarrow D_{s}^{-} \pi^{+}$decays in the different datasets. Only statistical errors are quoted.

\begin{tabular}{c|c|c|c} 
& hbot0h & hbot1i & combined \\
\hline \hline Events & $567 \pm 31$ & $589 \pm 32$ & $1153 \pm 45$ \\
Mass $\left[\mathrm{MeV} / \mathrm{c}^{2}\right]$ & $5275.7 \pm 1.3$ & $5279.6 \pm 1.5$ & $5277.6 \pm 1.0$ \\
Width $\left[\mathrm{MeV} / \mathrm{c}^{2}\right]$ & $21.6 \pm 1.2$ & $24.8 \pm 1.4$ & $23.2 \pm 0.9$
\end{tabular}

Table 5.7: Fit results for $B^{0} \rightarrow D^{-} \pi^{+}$decays in the different datasets. Only statistical errors are quoted. 


\subsection{Monte Carlo Validation}

The biggest challenge in comparing data to Monte Carlo is doing proper sideband subtraction in the samples. The problem comes from the fact that at masses just below the signal peak, which is where one would usually sample the low-mass sideband, there are large reflection structures. We need to use input from Sections 5.2 and 5.2.6 to solve this problem.

Based on the findings of these sections, we assume that the background under the signal peak is completely combinatorial, which means that it is equivalent to the background we will find in the high-mass sideband. The low-mass sideband has an additional component which comes from $B \rightarrow D X$-type decays which are not present under the signal peak, and should therefore not be directly subtracted from the signal distributions without somehow taking into account the distributions of $B \rightarrow D X$-type backgrounds. Furthermore, the shape of the background distribution can be described with an exponential function. We need to take into account that the background level in the high-mass sideband and signal regions are not the same. We therefore fit the mass spectrum in the high-mass sideband with an exponential distribution and extrapolate the fit result into the signal region. The integral of the extrapolated exponential across the signal region provides an estimate of the background level in the signal region.

Figure 5-10 shows the definitions of the signal and high-mass sideband regions for our comparisons. Since the mass of the $B^{+}$and $B^{0}$ mesons are virtually the same, we can use the same mass regions in both cases. The signal region is defined as $5.20 \mathrm{GeV} / \mathrm{c}^{2}<\mathrm{m}(\mathrm{B})<5.35 \mathrm{GeV} / \mathrm{c}^{2}$. The high-mass sideband is defined as $5.425 \mathrm{GeV} / \mathrm{c}^{2}<\mathrm{m}(\mathrm{B})<5.575 \mathrm{GeV} / \mathrm{c}^{2}$. Figure $5-11$ shows fits of the high-mass tail to an exponential distribution. The fit results were used to obtain normalization scale factors for the sideband subtraction procedure.

The simulation is run for the different representative runs for the pre-shutdown

and post-shutdown data. To evaluate the validity of the Monte Carlo simulation, a number of variables have to be compared. To check the validity of the underlying 
theory, production, and fragmentation it is sufficient to study the hadron $p_{T}$ and $\eta$ distributions. To check the validity of the trigger simulation, the number of trigger pairs, the trigger track $p_{T}, d_{0}$ and $\phi_{0}$ and the trigger pair $\sum p_{T}, \Delta \phi$ and $L_{x y}$ have to be investigated. Those are the quantities relevant to the trigger decision. To validate the offline reconstruction, the $p_{T}\left(\pi_{B}\right), p_{T}(D), p_{T}(B), \phi_{0}(B), \eta(B), \chi_{r \phi}^{2}(D)$, $\chi_{r \phi}^{2}(B), L_{x y}(B \rightarrow D), L_{x y}(B), L_{x y}(D), \Delta R\left(D, \pi_{B}\right)$ and $d_{0}(B)$ are also compared. These are the variables which are used in the selection. It is important to note that even though we compare distributions between data and Monte Carlo separately for both our high-statisticis samples, our measurement is only sensitive to differences in relative efficiencies. Even if there are discrepancies between a given distribution and its Monte Carlo, as long as the discrepancy is consisistent between different $B$ species, it cancels in the ratio.

In the following subsections we perform a detailed comparison of the Monte Carlo simulation to the reconstructed high statistics decays $B^{+} \rightarrow \overline{D^{0}} \pi^{+}$and $B^{0} \rightarrow D^{-} \pi^{+}$ . In all pictures sideband subtraction is applied to single out only the behavior of the real $B^{0}$ signal. To avoid trigger volunteer candidates in the sample the trigger is confirmed on each candidate separately. Since the winter and summer datasets have slightly different properties they are studied separately. The Monte Carlo candidates are properly weighted according to Tables 5.3 to account for the integrated luminosity in each sub-period. In the Figures 5-12 through 5-43, the comparison between sideband subtracted data and Monte Carlo simulation is summarized. Good agreement is observed in most distributions. The largest discrepancies are present in the $\chi^{2}(B)$ distribution in Figures 5-20 and 5-21. 


\begin{tabular}{c|c|c} 
Run Range & Representative & $\mathcal{L}\left(\mathrm{pb}^{-1}\right)$ \\
\hline \hline $138809-143000$ & 141508 & 3.4 \\
$143001-146000$ & 144578 & 3.7 \\
$146001-149559$ & 146920 & 7.2 \\
$150010-151117$ & 150799 & 1.9 \\
$151118-152668$ & 151513 & 10.7 \\
$152669-152953$ & 152747 & 2.3 \\
$152953-156487$ & 153618 & 32.69 \\
\hline $159603-160541$ & 160230 & 6.541 \\
$160542-161013$ & 160823 & 6.396 \\
$161014-161633$ & 161379 & 6.649 \\
$161634-162178$ & 161379 & 6.535 \\
$162179-162480$ & 162423 & 6.604 \\
$162481-162825$ & 162498 & 6.489 \\
$162826-163012$ & 162857 & 6.436 \\
$163013-164200$ & 163064 & 6.429
\end{tabular}

Table 5.8: Run ranges, representative runs and luminosity per run range for the hbot $0 \mathrm{~h}$ and hbot $1 \mathrm{i}$ datasets. The total integrated luminosity for the sample is 115 $\mathrm{pb}^{-1}$. 

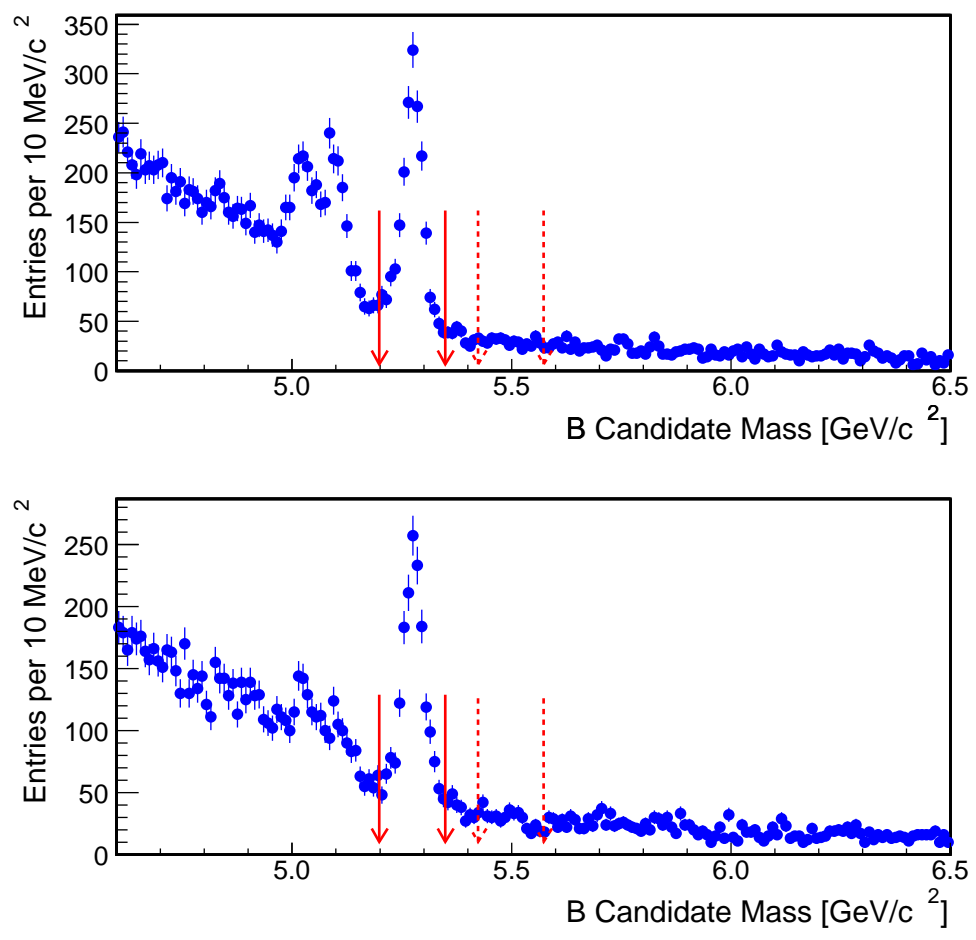

Figure 5-10: Signal and high-mass sideband region definitions for sideband subtraction. The top plot shows the mass spectrum for the $B^{+}$decays, and the bottom plot shows the same distribution for the $B^{0}$ decays. The signal region is marked with solid arrows, and the sideband with dashed arrows. 

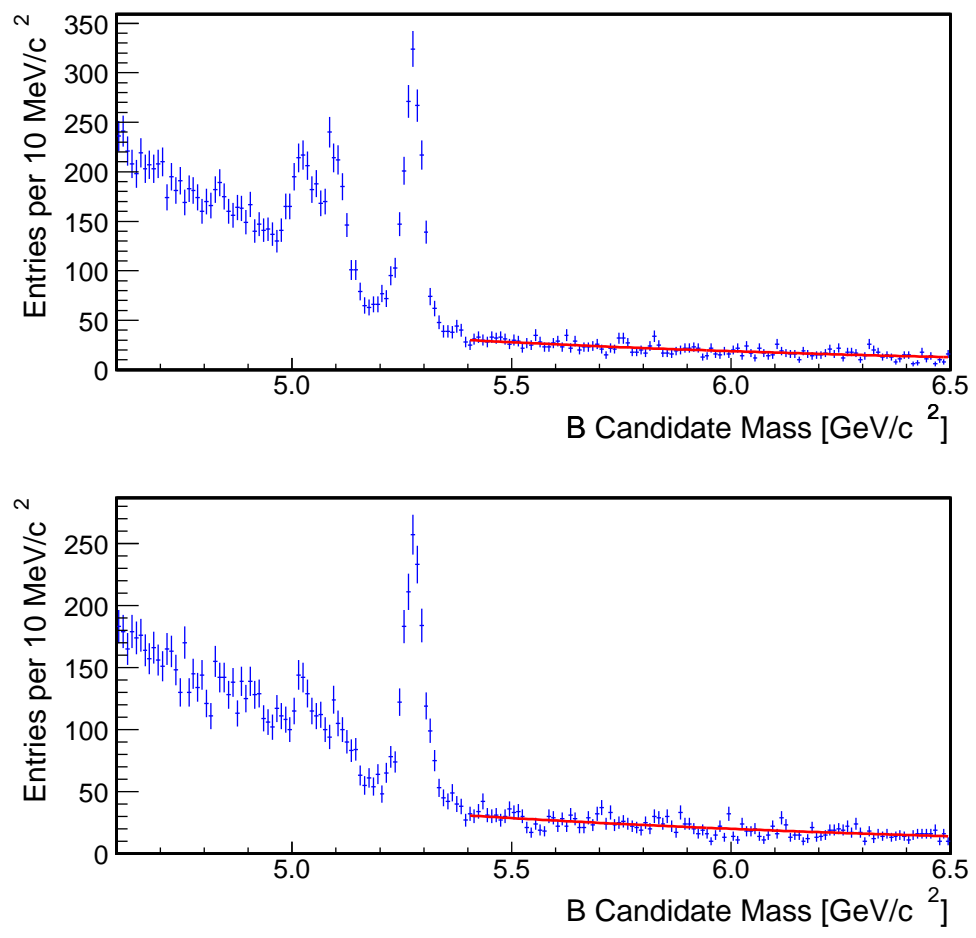

Figure 5-11: Fit of the high-mass background to an exponential distribution. The parameters of the exponential fit are used to determine the normalization factor used in sideband subtraction. The top plot shows the exponential fit for $B^{+}$decays, and the bottom plot shows the exponential fit for $B^{0}$ decays. 

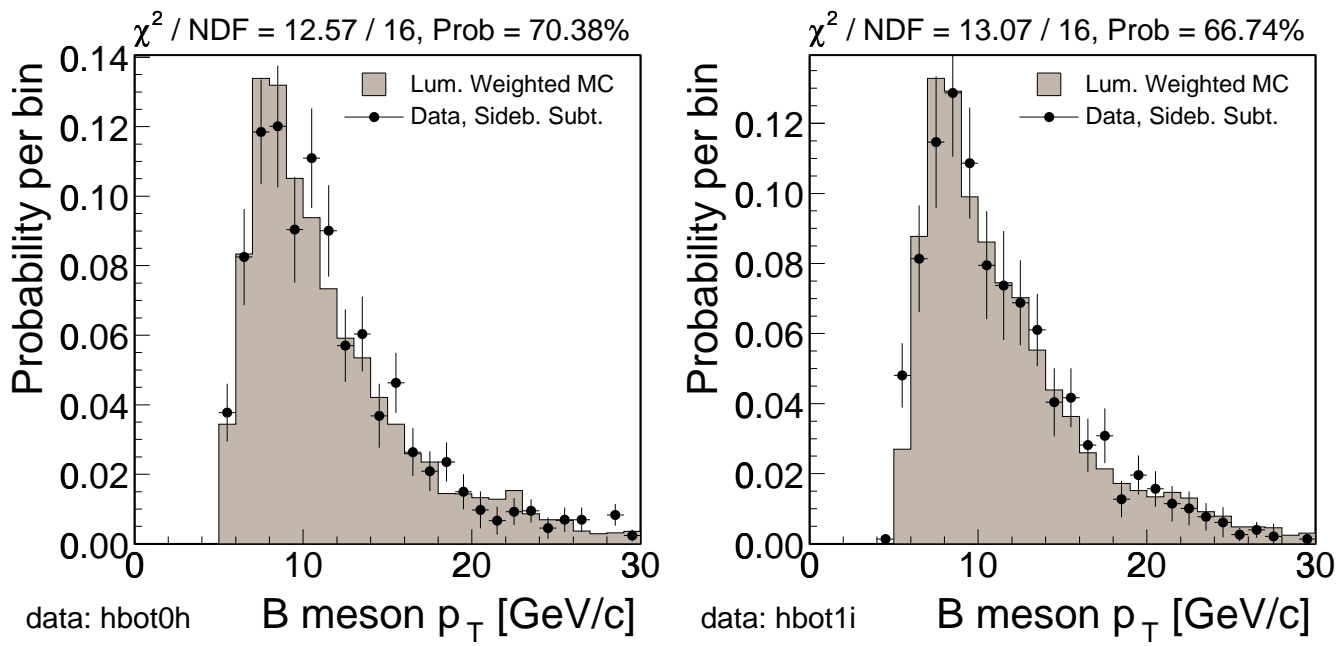

Figure 5-12: Comparison of Data and Monte Carlo distributions of the $p_{T}(B)$ for $B^{+}$ decays.
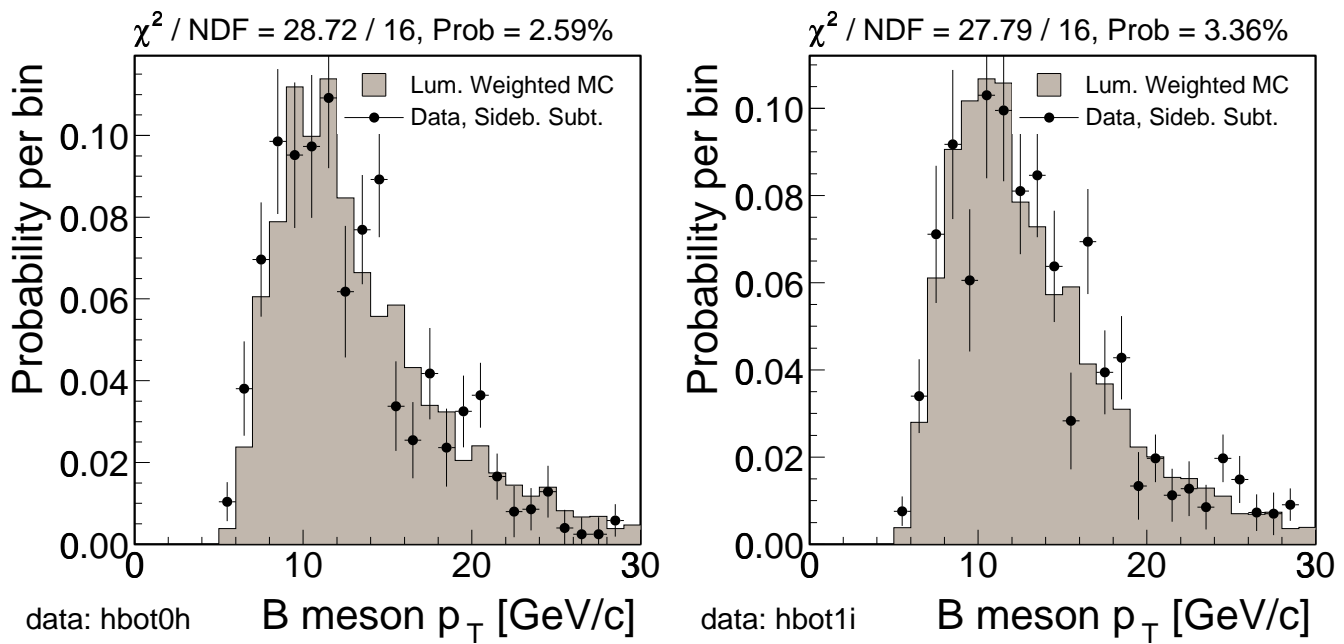

Figure 5-13: Comparison of Data and Monte Carlo distributions of the $p_{T}(B)$ for $B^{0}$ decays. 

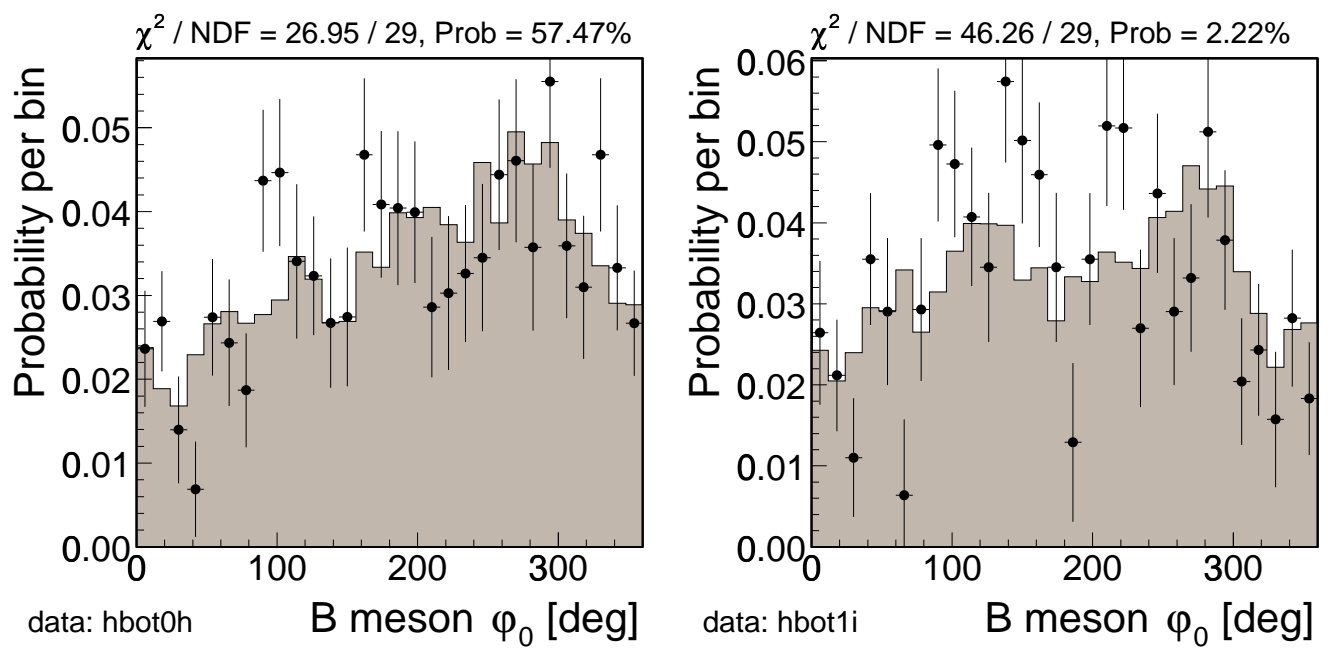

Figure 5-14: Comparison of Data and Monte Carlo distributions of the $\phi_{0}(B)$.
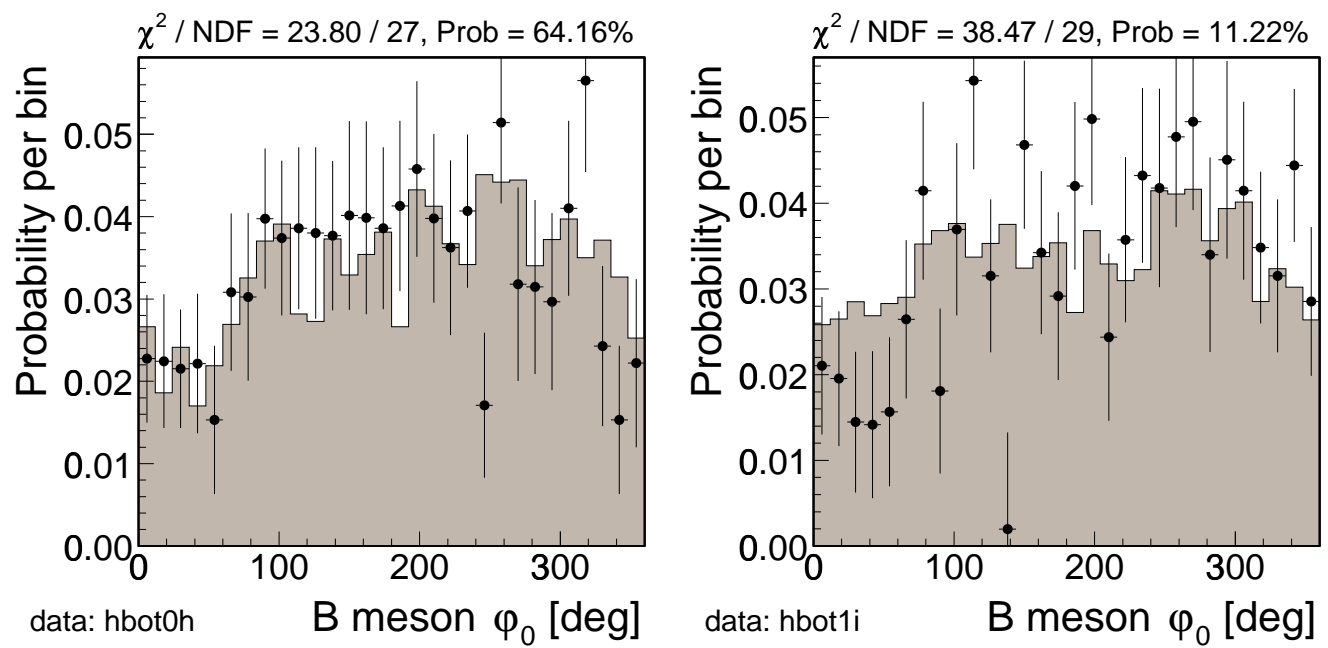

Figure 5-15: Comparison of Data and Monte Carlo distributions of the $\phi_{0}(B)$. 

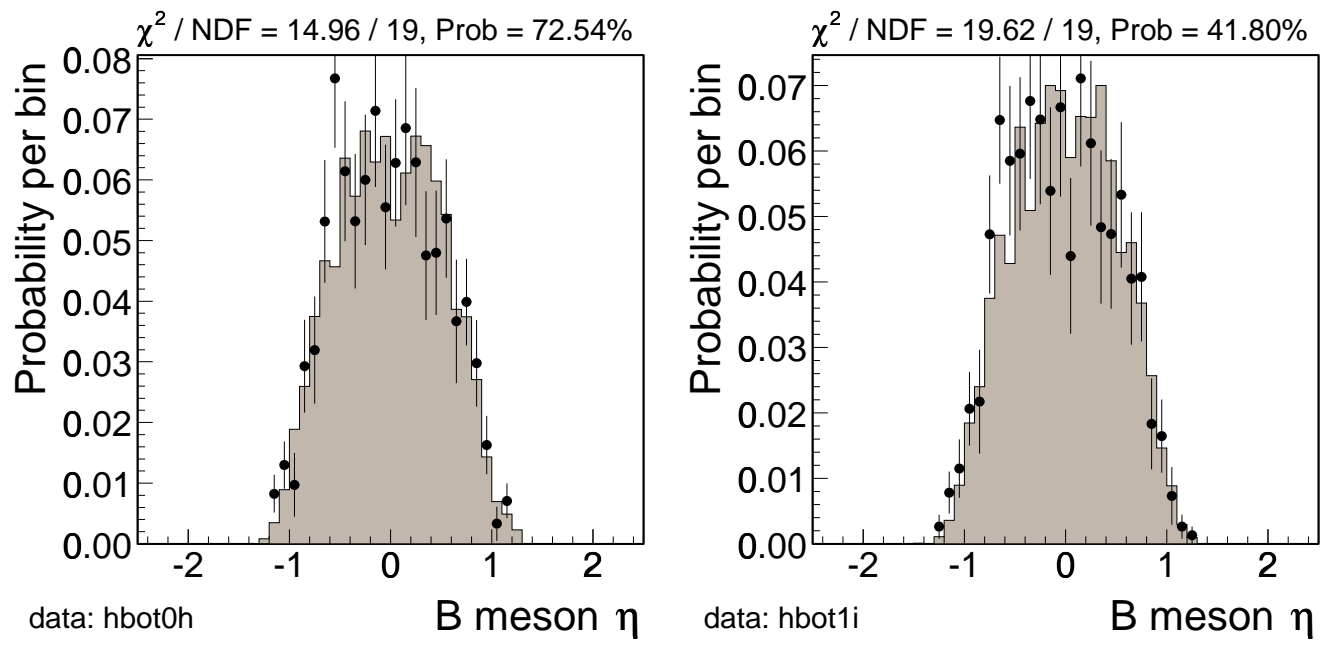

Figure 5-16: Comparison of Data and Monte Carlo distributions of the $\eta(B)$ for $B^{+}$ decays.
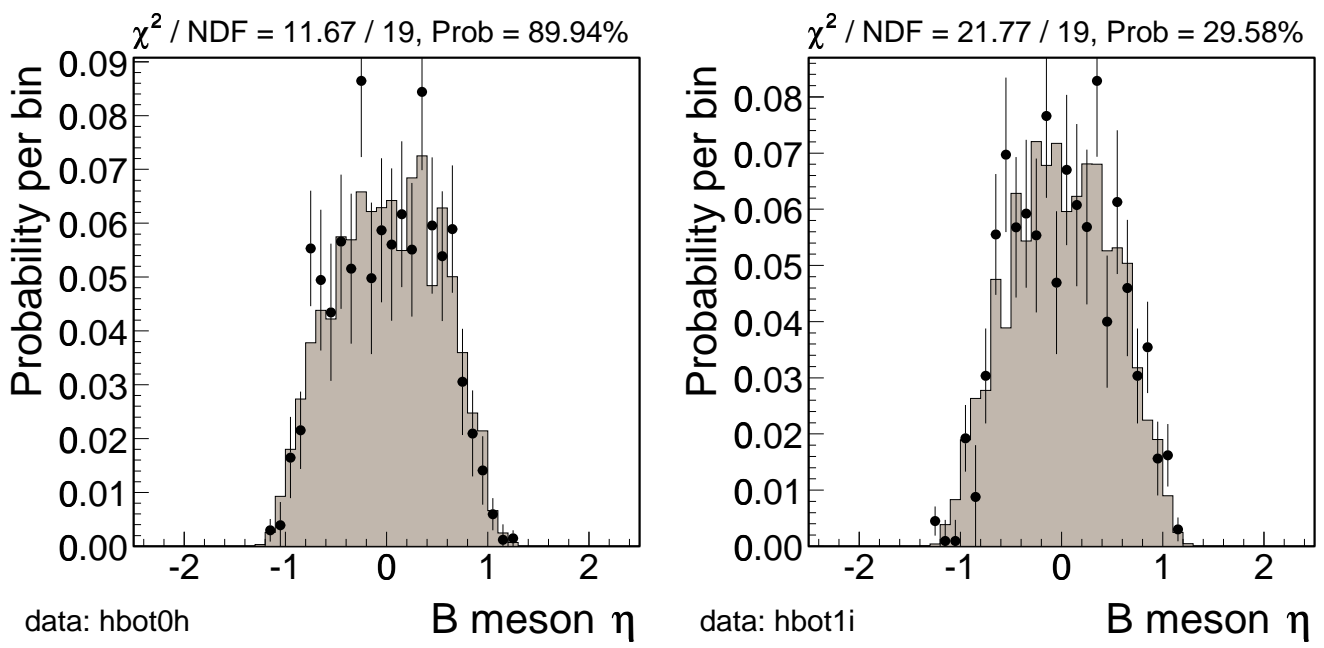

Figure 5-17: Comparison of Data and Monte Carlo distributions of the $\eta(B)$ for $B^{0}$ decays. 

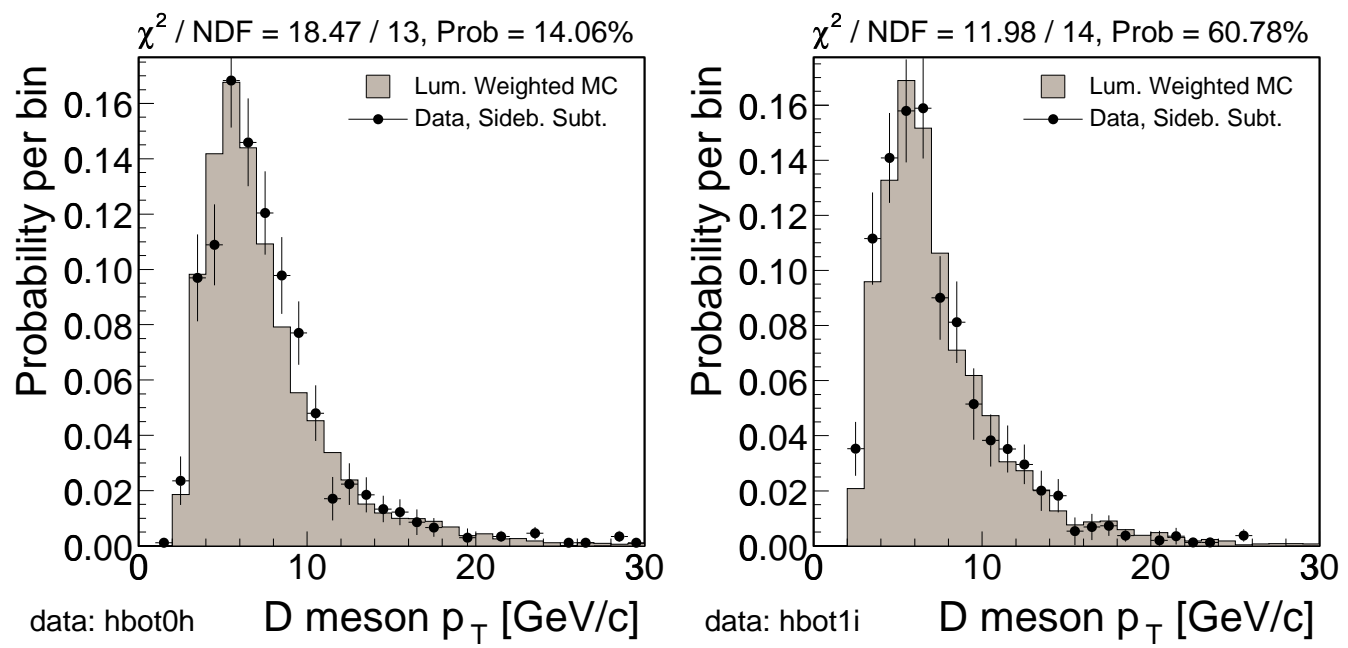

Figure 5-18: Comparison of Data and Monte Carlo distributions of the $p_{T}(D)$.
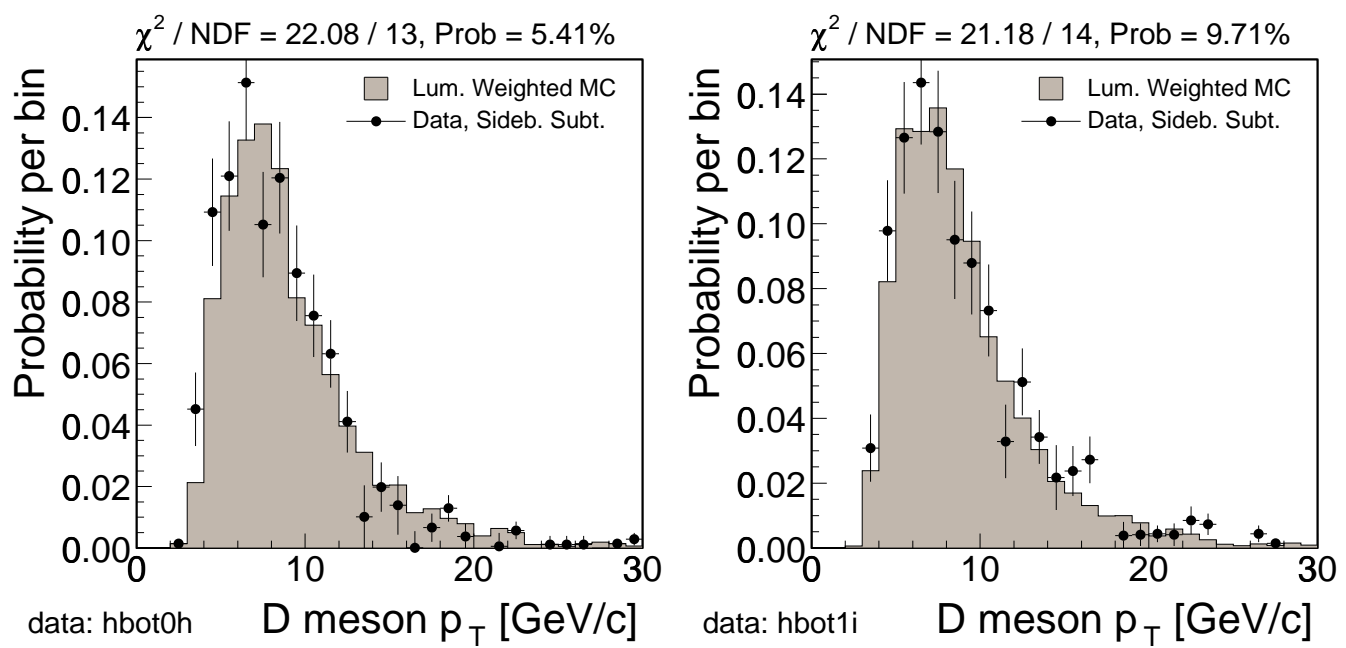

Figure 5-19: Comparison of Data and Monte Carlo distributions of the $p_{T}(D)$. 

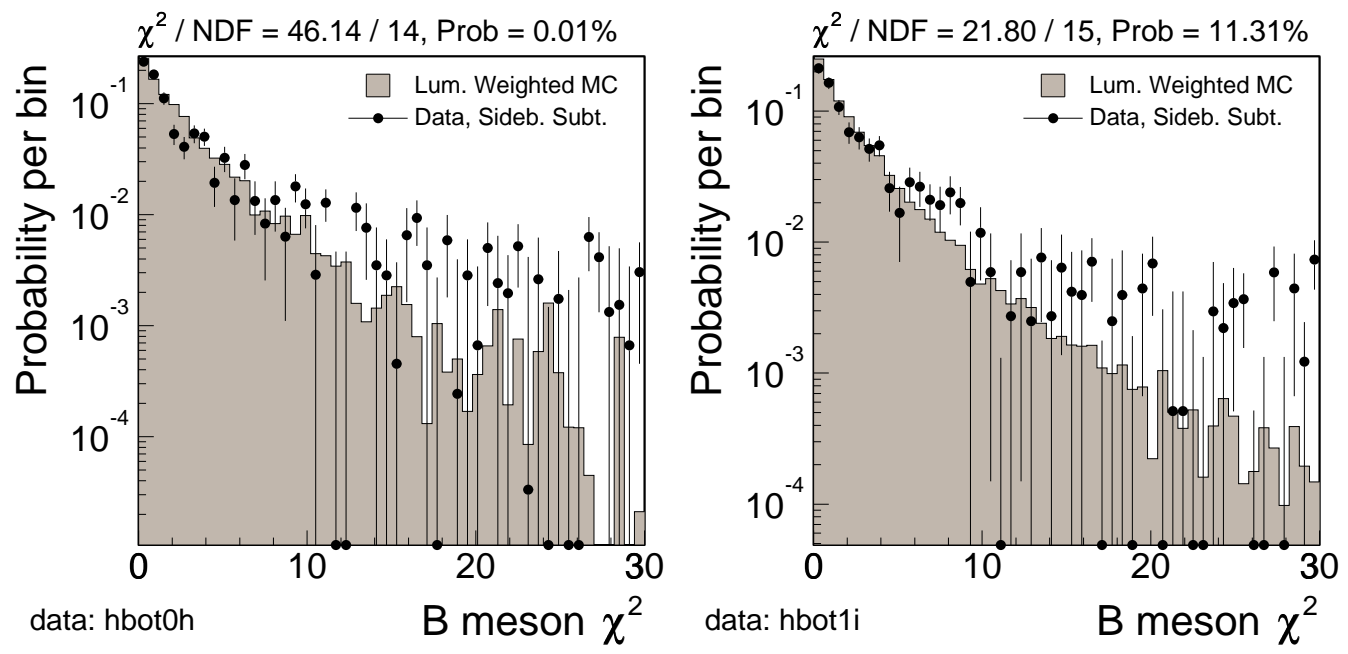

Figure 5-20: Comparison of Data and Monte Carlo distributions of the $\chi^{2}(B)$.
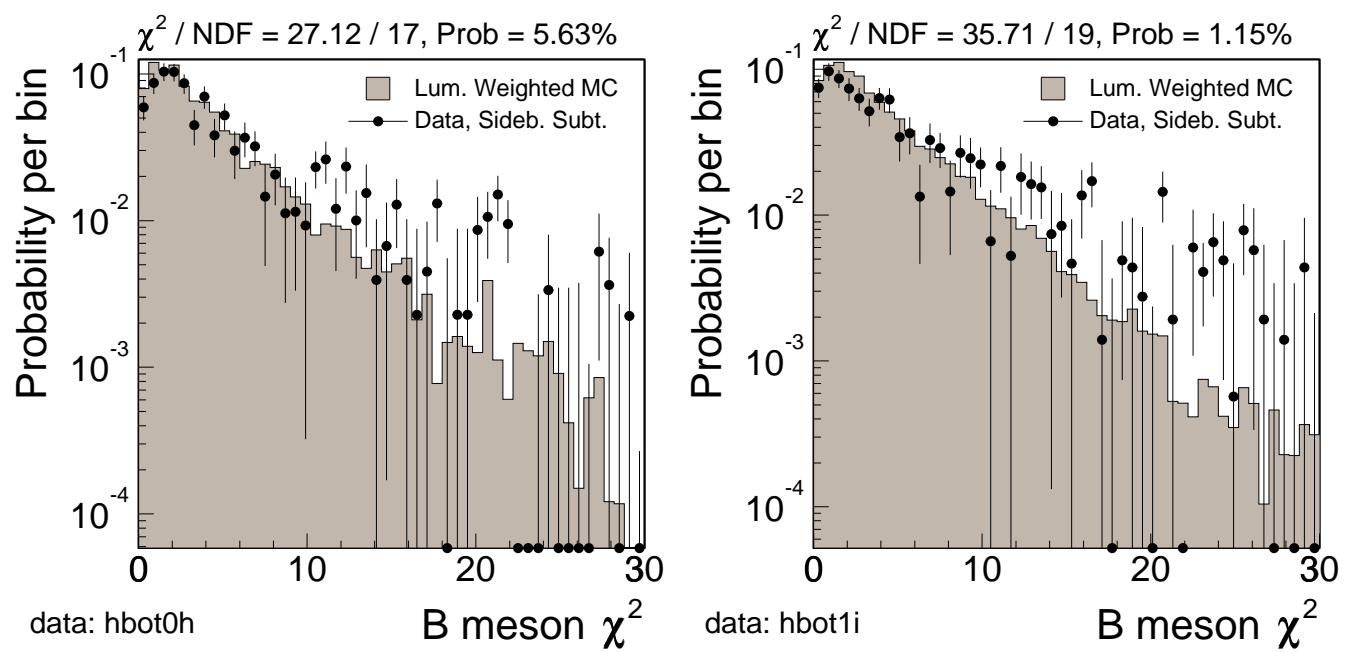

Figure 5-21: Comparison of Data and Monte Carlo distributions of the $\chi^{2}(B)$. 

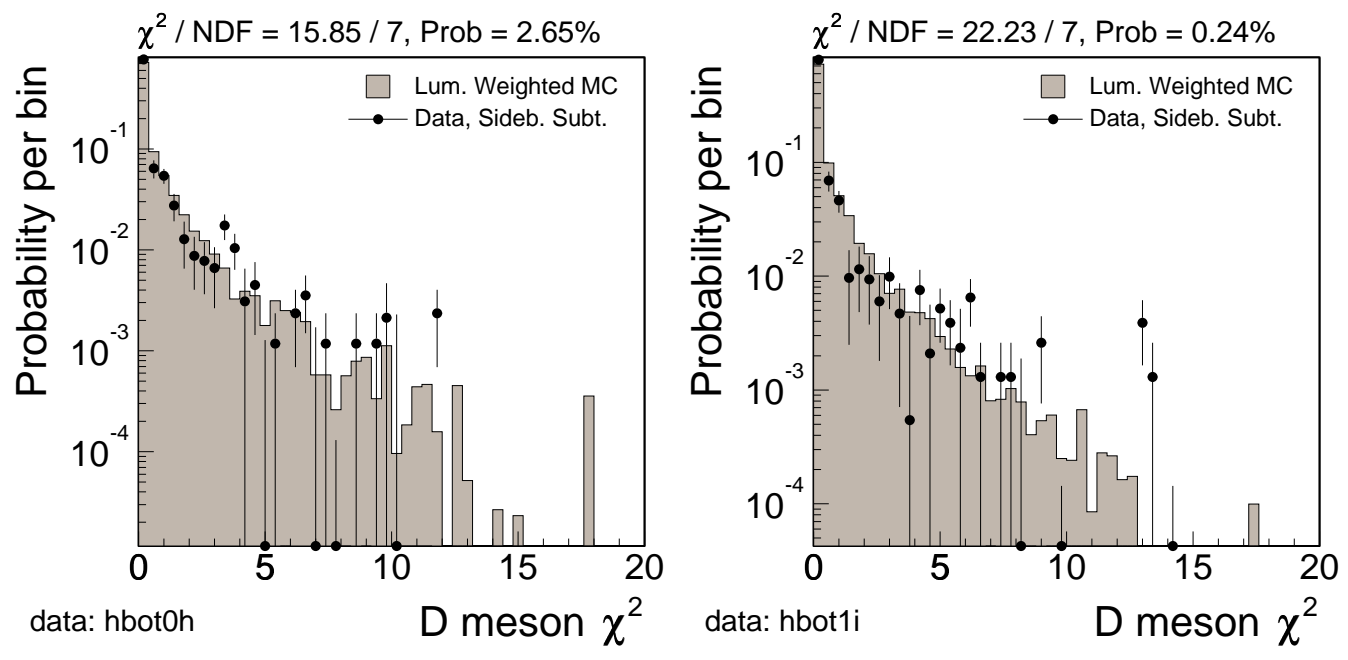

Figure 5-22: Comparison of Data and Monte Carlo distributions of the $\chi^{2}(D)$.
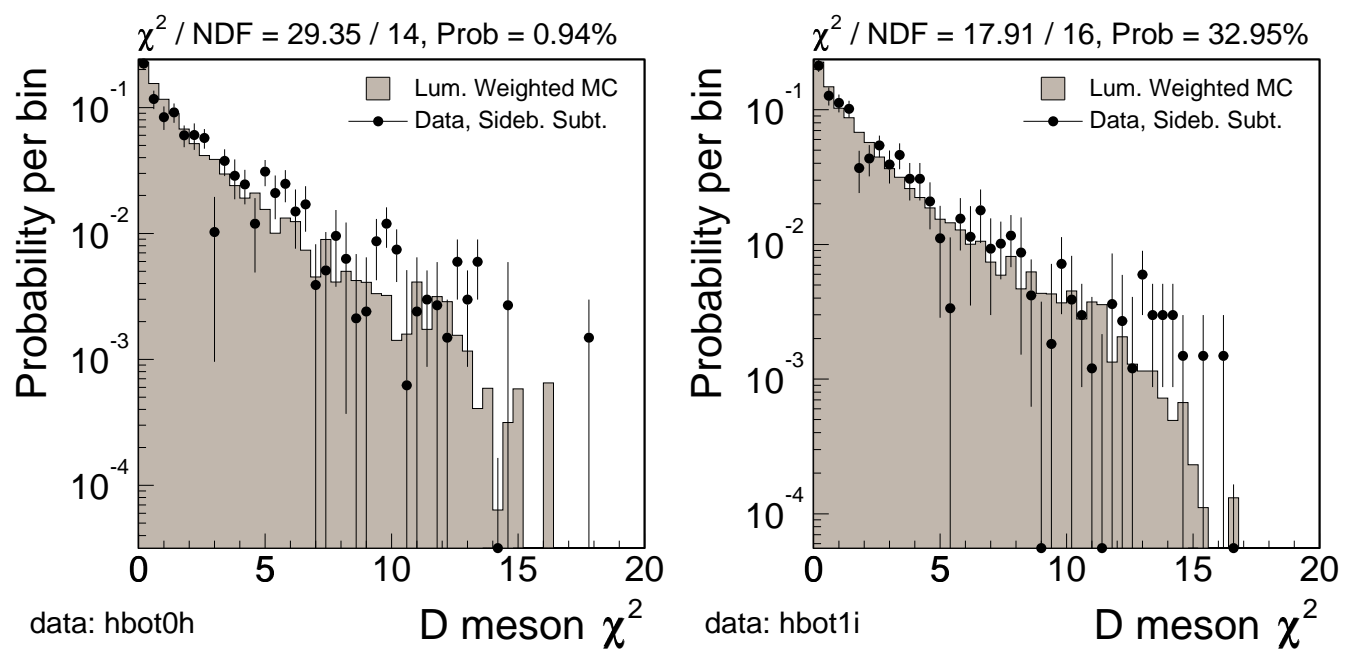

Figure 5-23: Comparison of Data and Monte Carlo distributions of the $\chi^{2}(D)$. 

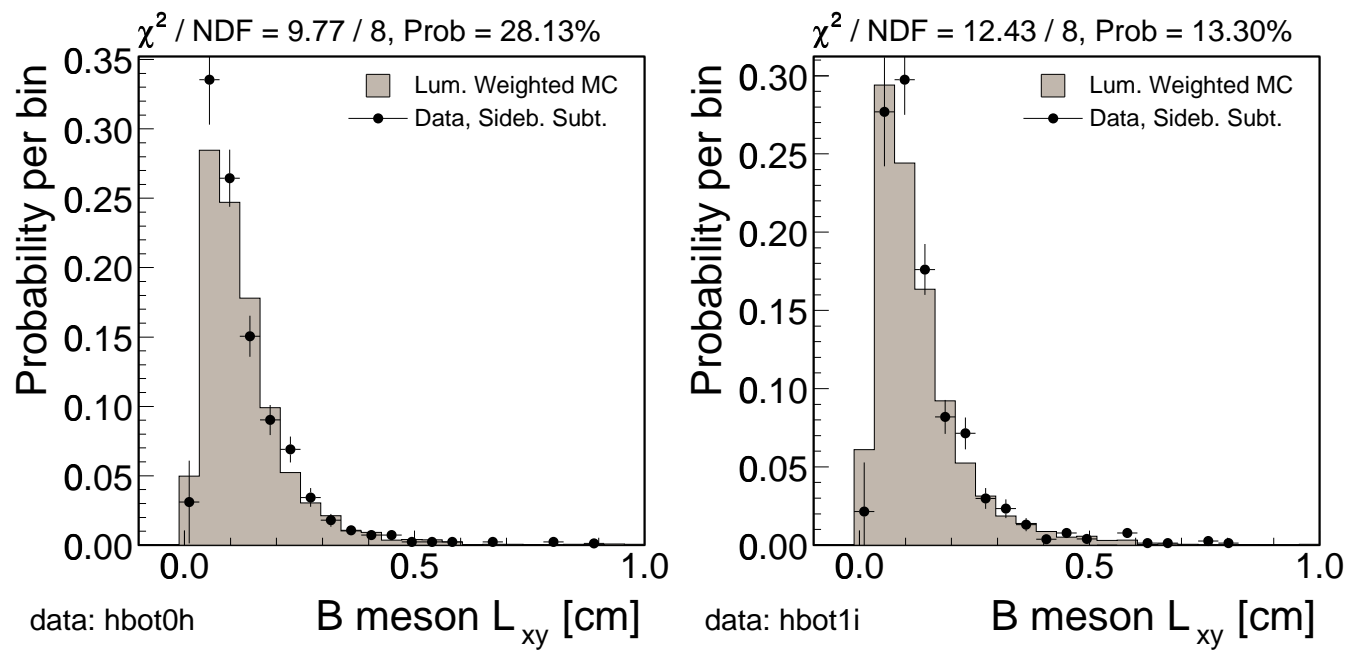

Figure 5-24: Comparison of Data and Monte Carlo distributions of the $L_{x y}(B)$.
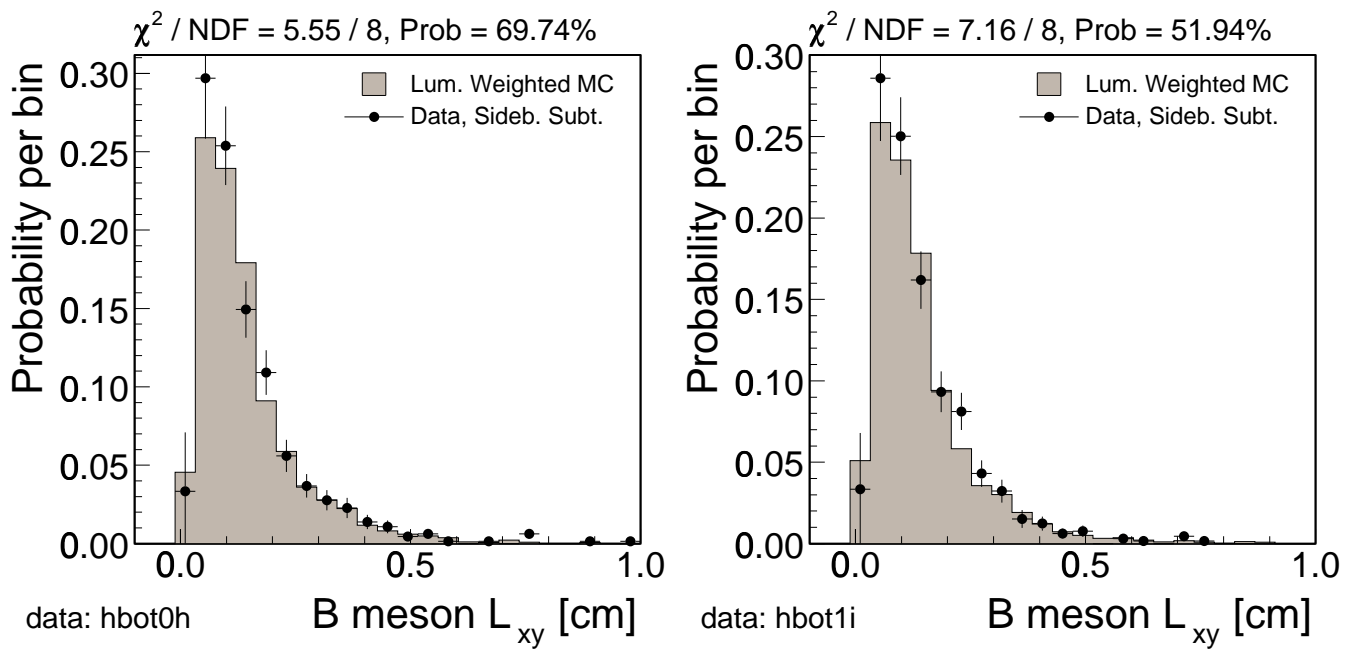

Figure 5-25: Comparison of Data and Monte Carlo distributions of the $L_{x y}(B)$. 

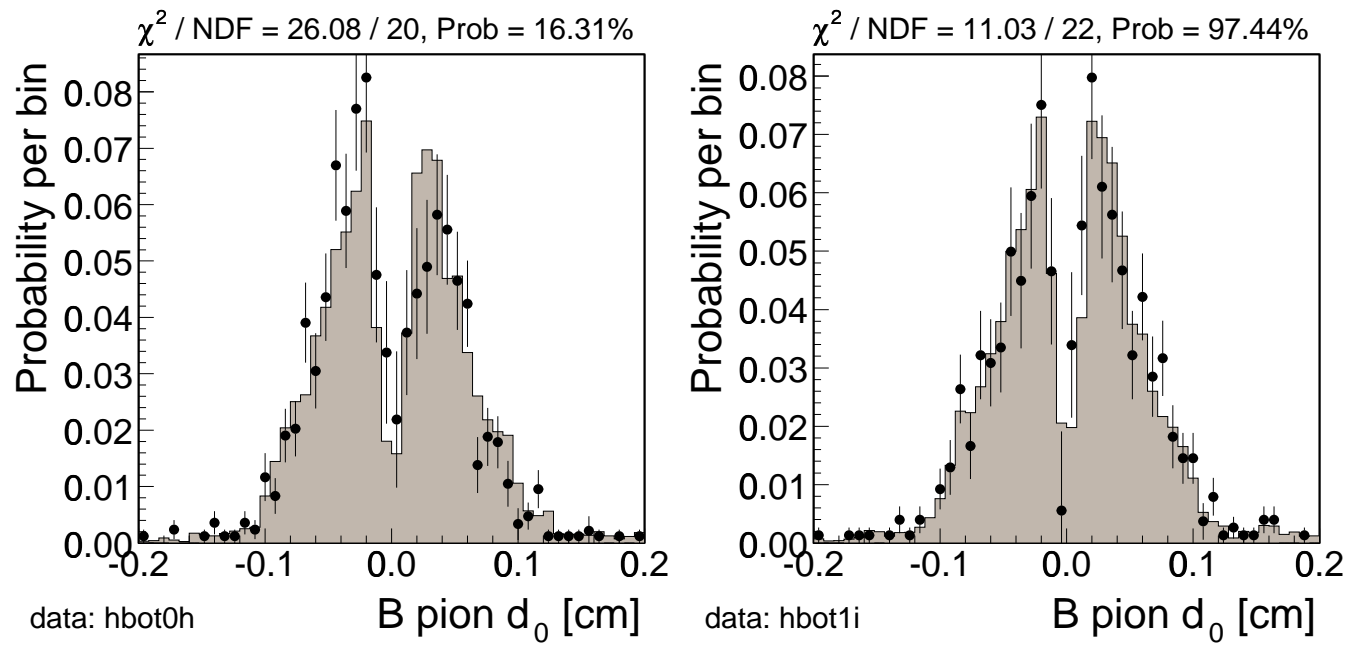

Figure 5-26: Comparison of Data and Monte Carlo distributions of the $d_{0}\left(\pi_{B}\right)$.
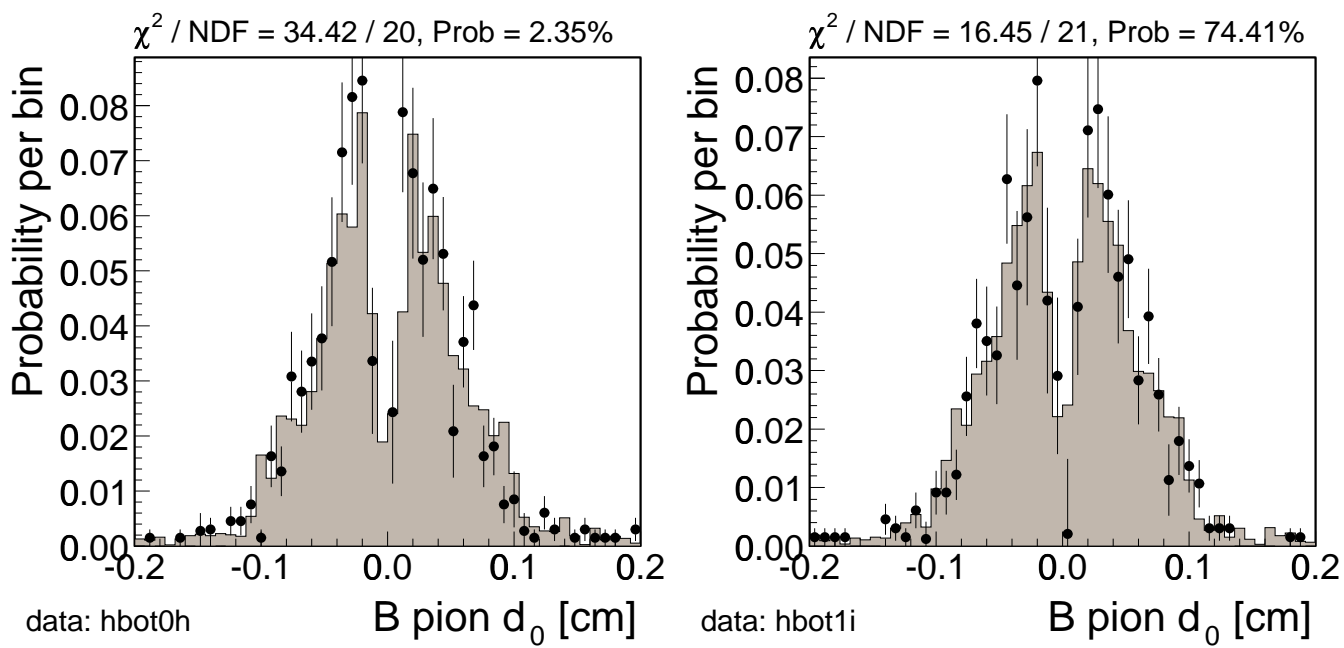

Figure 5-27: Comparison of Data and Monte Carlo distributions of the $d_{0}\left(\pi_{B}\right)$. 

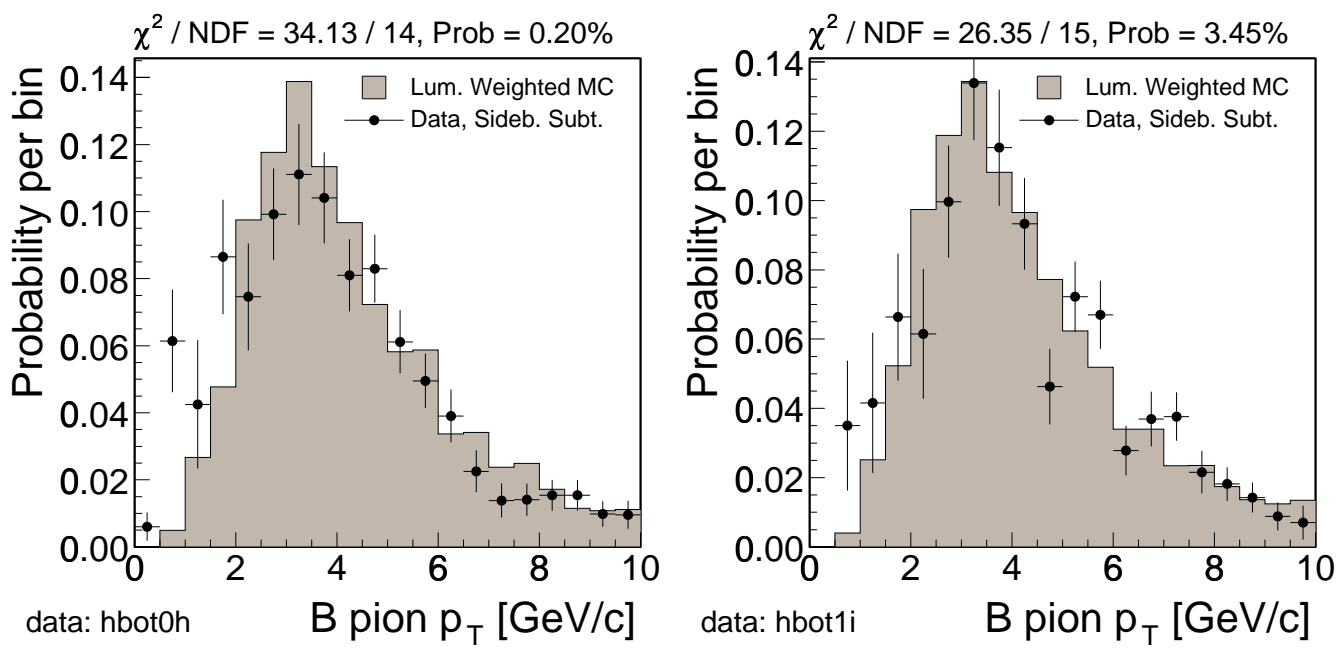

Figure 5-28: Comparison of Data and Monte Carlo distributions of the $p_{T}\left(\pi_{B}\right)$.
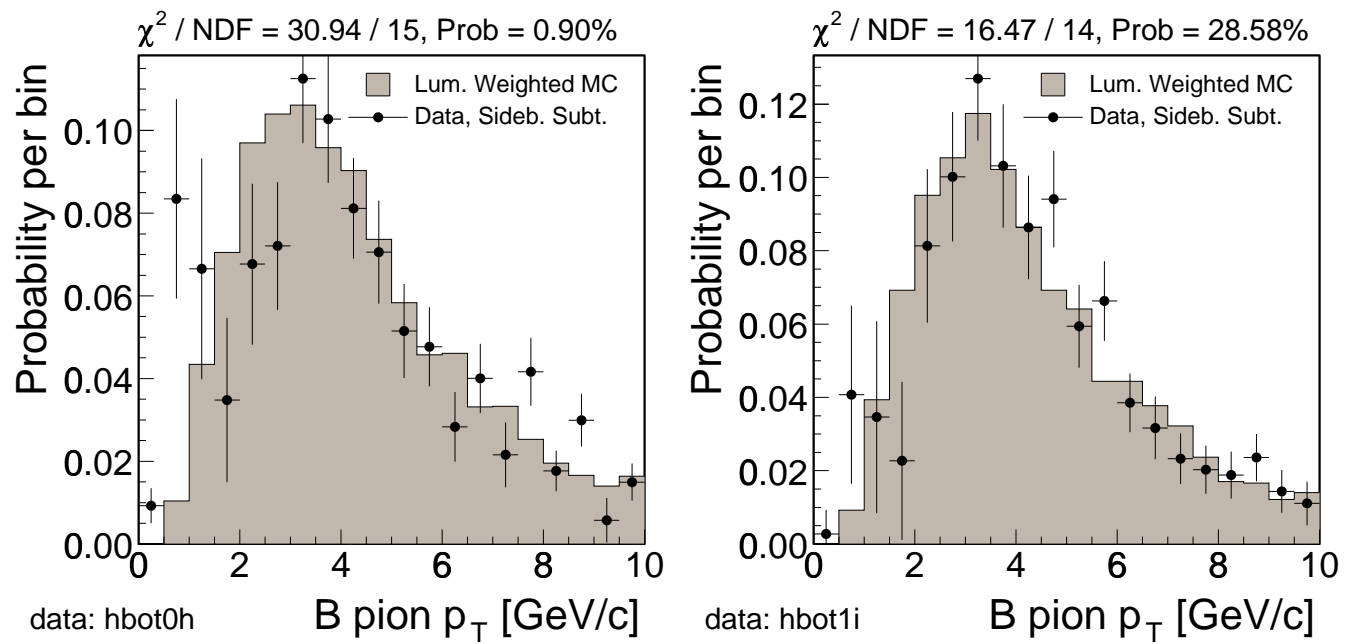

Figure 5-29: Comparison of Data and Monte Carlo distributions of the $p_{T}\left(\pi_{B}\right)$. 

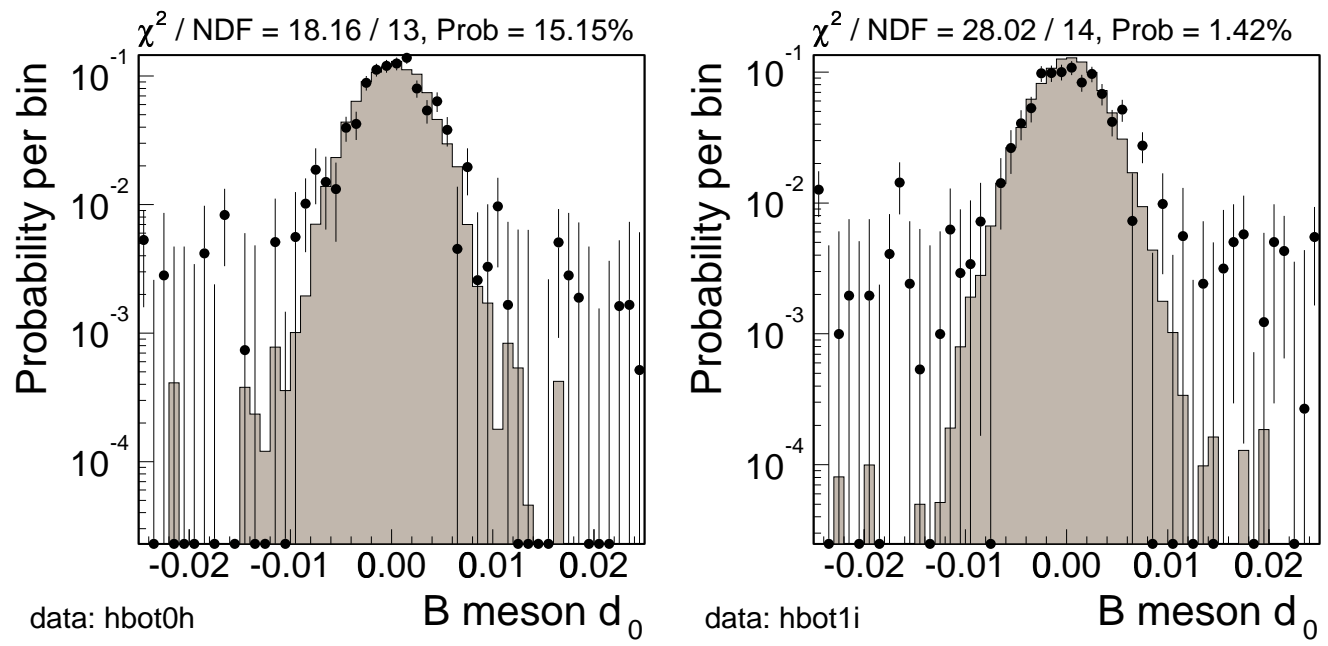

Figure 5-30: Comparison of Data and Monte Carlo distributions of the $d_{0}(B)$ for $B^{+}$ decays.
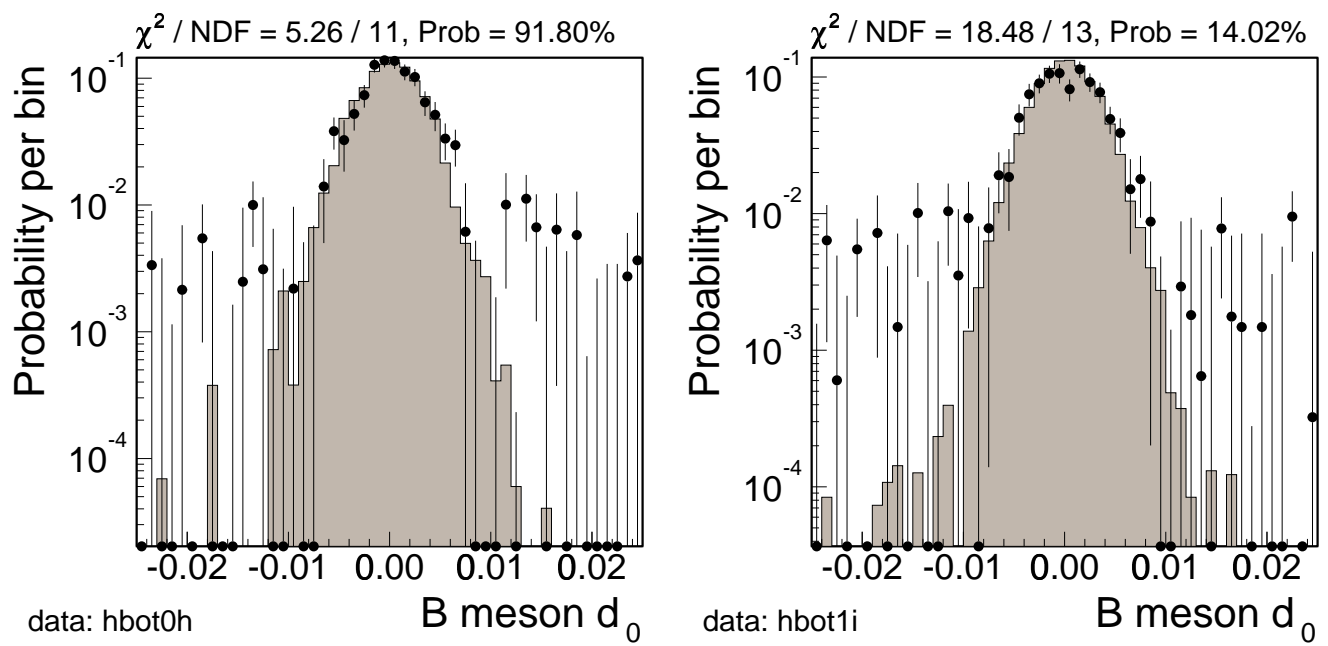

Figure 5-31: Comparison of Data and Monte Carlo distributions of the $d_{0}(B)$ for $B^{0}$ decays. 

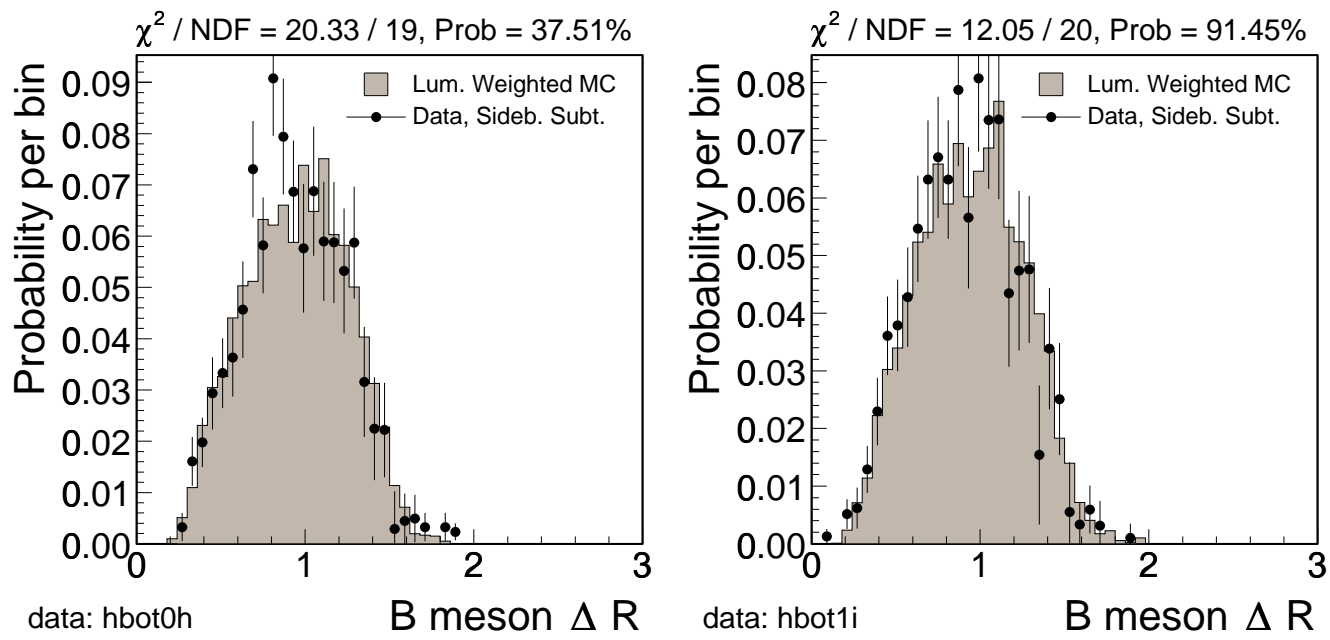

Figure 5-32: Comparison of Data and Monte Carlo distributions of the $\Delta R\left(D, \pi_{B}\right)$ for $B^{+}$decays.
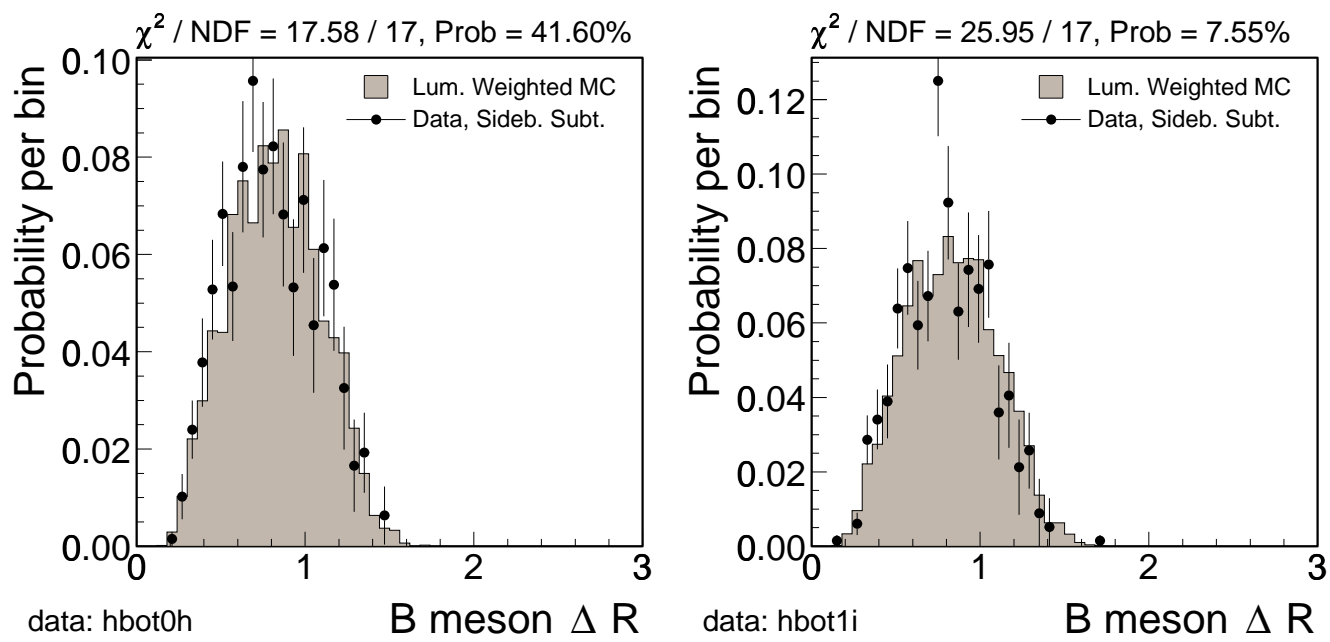

Figure 5-33: Comparison of Data and Monte Carlo distributions of the $\Delta R\left(D, \pi_{B}\right)$ for $B^{0}$ decays. 

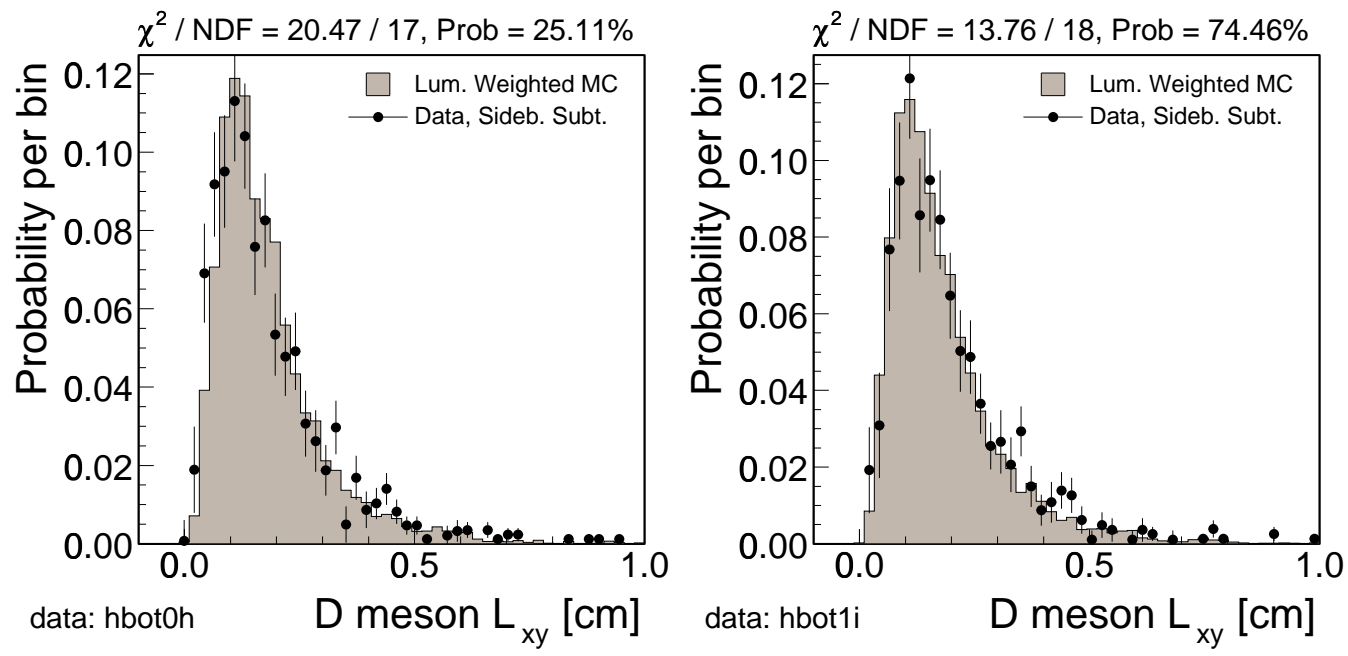

Figure 5-34: Comparison of Data and Monte Carlo distributions of the $L_{x y}(D)$ with respect to the primary vertex.
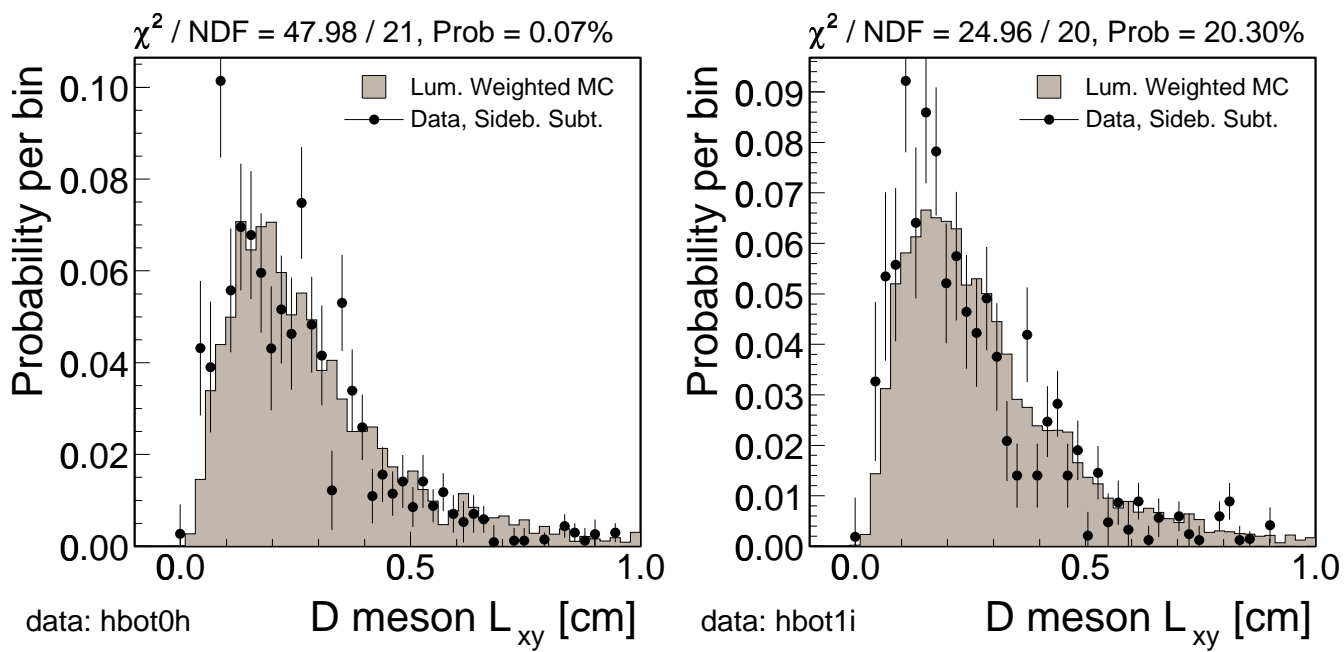

Figure 5-35: Comparison of Data and Monte Carlo distributions of the $L_{x y}(D)$ with respect to the primary vertex. 

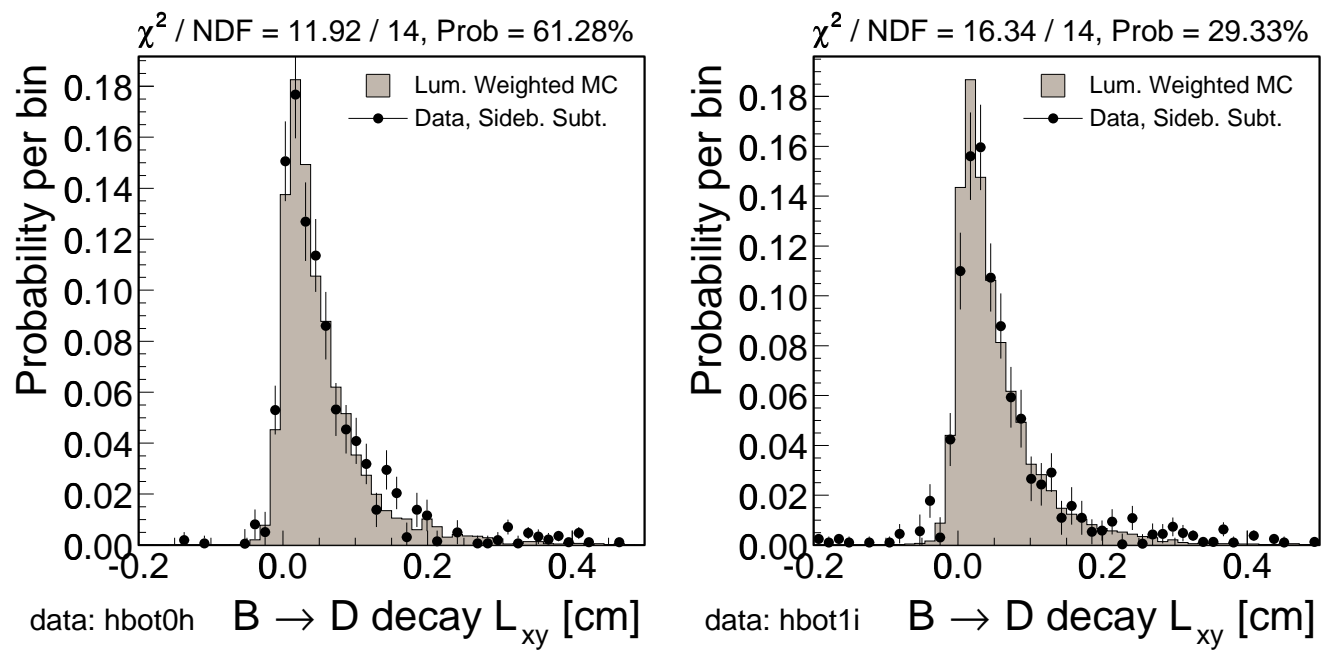

Figure 5-36: Comparison of Data and Monte Carlo distributions of the $L_{x y}(D)$.
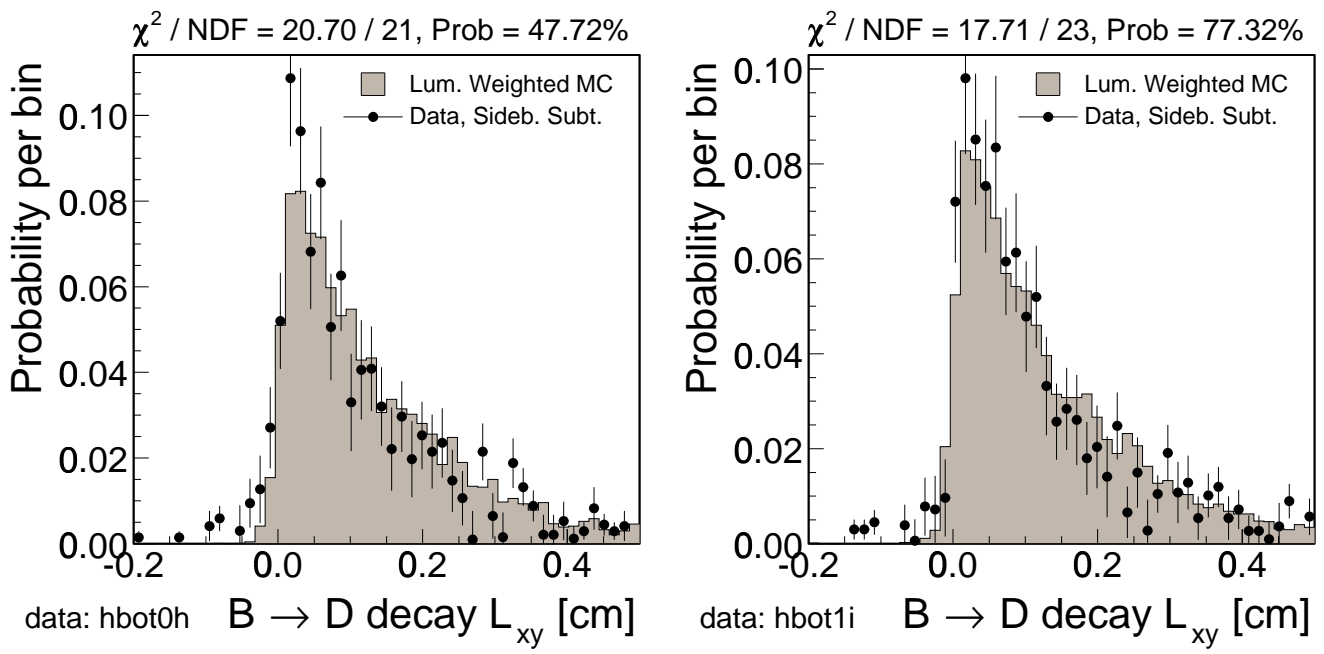

Figure 5-37: Comparison of Data and Monte Carlo distributions of the $L_{x y}(D)$. 

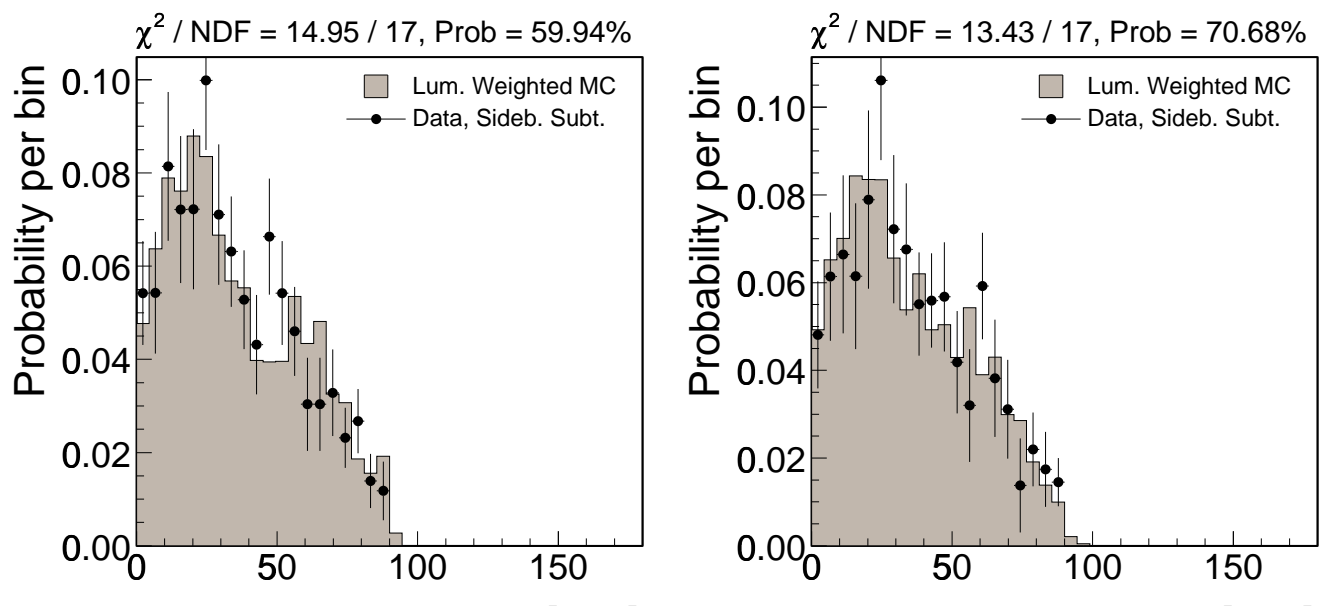

data: hbot0h Trig. pair $\Delta \varphi[\mathrm{deg}]$

data: hbot1i Trig. pair $\Delta \varphi[$ deg]

Figure 5-38: Comparison of Data and Monte Carlo distributions of the $\Delta \phi$ between the two trigger tracks.
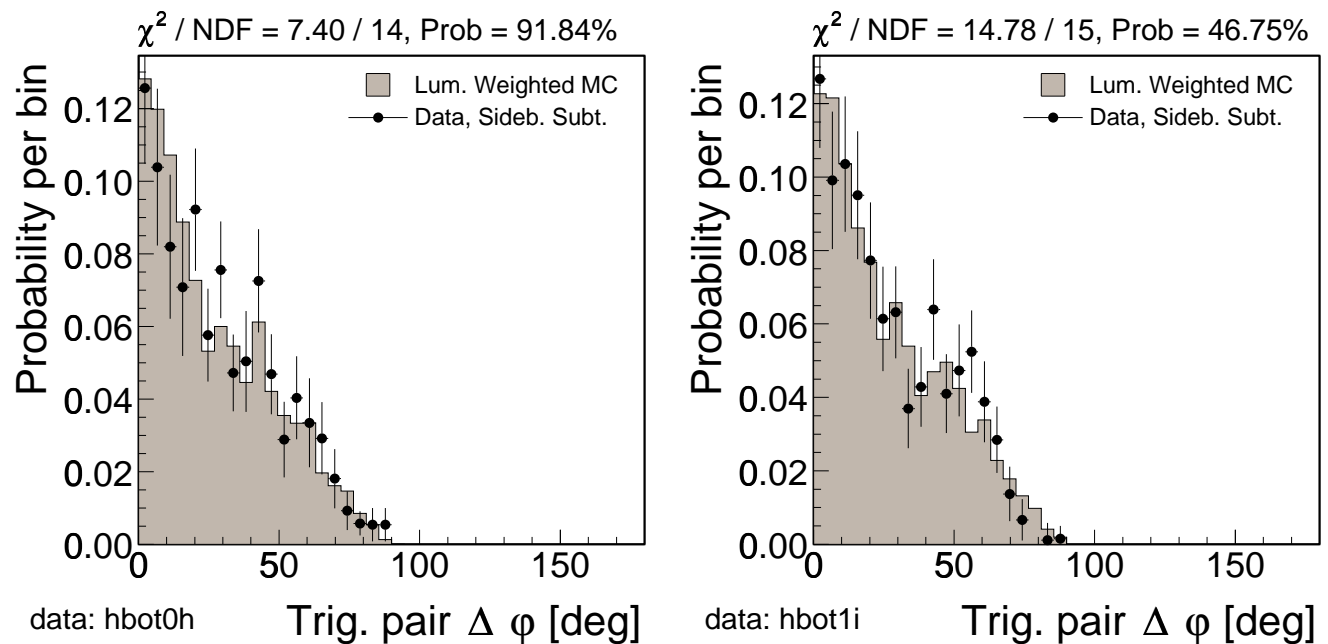

Figure 5-39: Comparison of Data and Monte Carlo distributions of the $\Delta \phi$ between the two trigger tracks. 

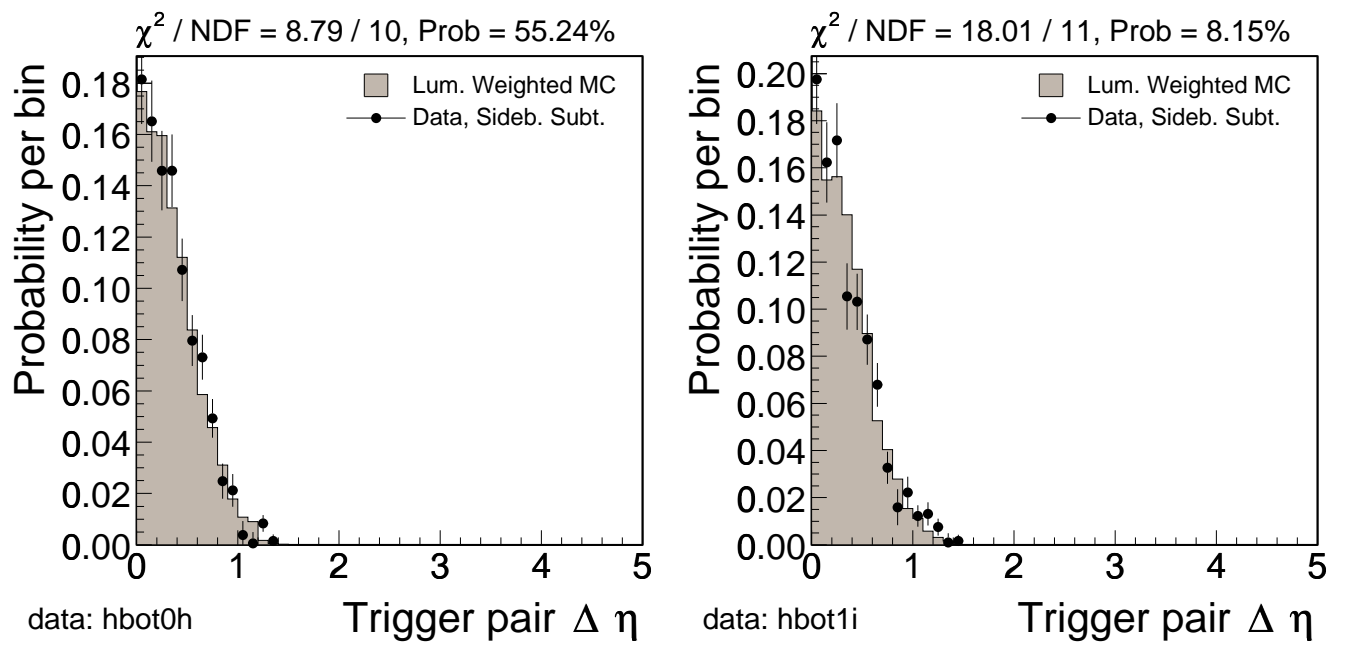

Figure 5-40: Comparison of Data and Monte Carlo distributions of the $\Delta \eta$ between the two trigger tracks.
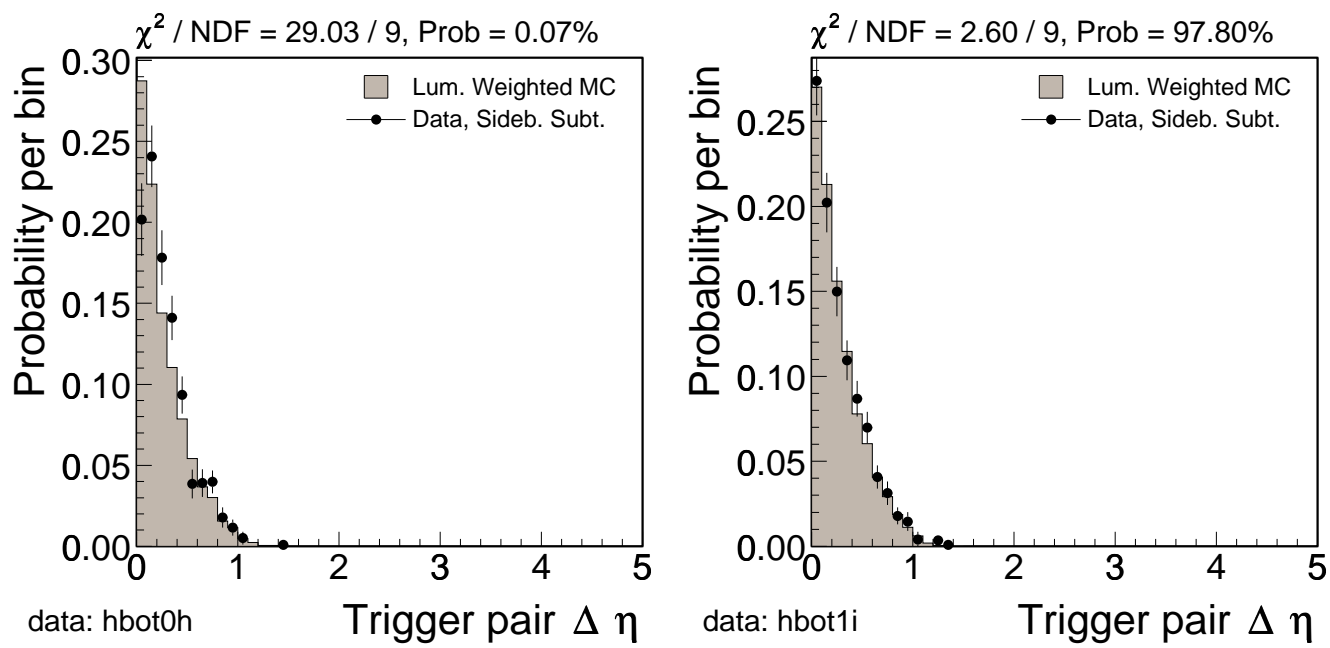

Figure 5-41: Comparison of Data and Monte Carlo distributions of the $\Delta \eta$ between the two trigger tracks. 

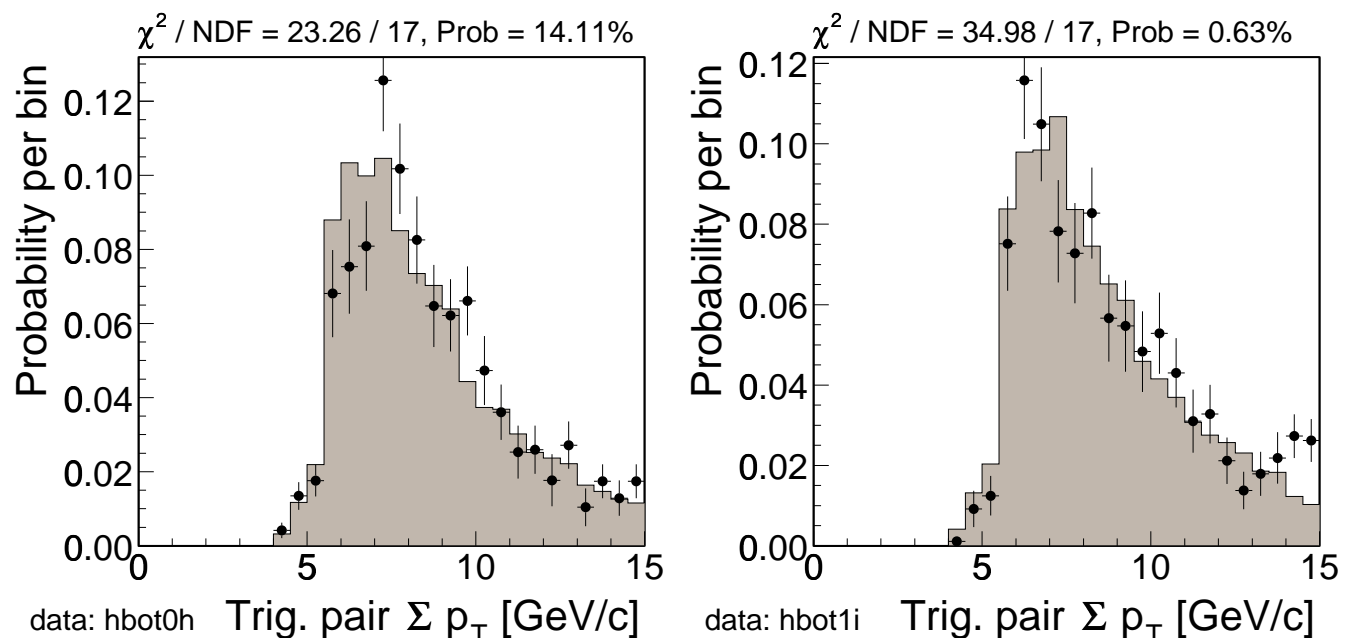

Figure 5-42: Comparison of Data and Monte Carlo distributions of the $\sum p_{T}$ of the two trigger tracks.
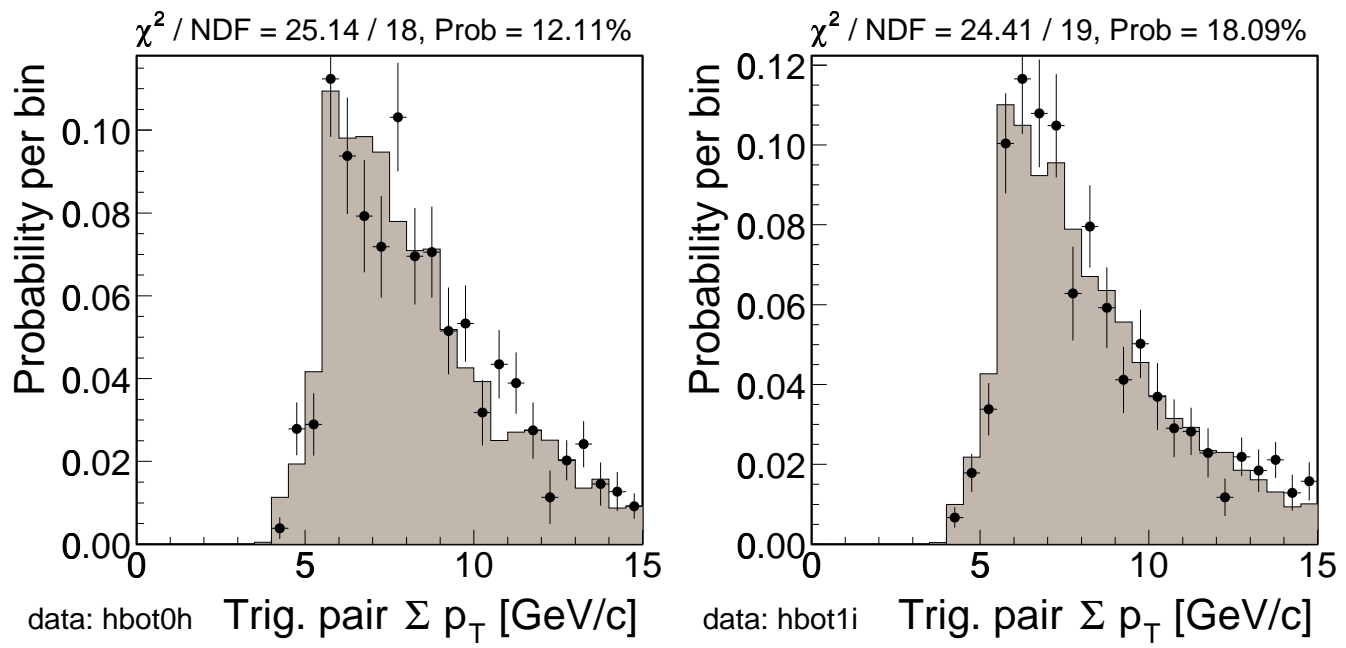

Figure 5-43: Comparison of Data and Monte Carlo distributions of the $\sum p_{T}$ of the two trigger tracks. 


\subsubsection{Reweighting the Monte Carlo}

A discrepancy is noticed between the transverse momentum spectrum of the Monte Carlo generated $B$ mesons and those reconstructed in data. We therefore apply one more correction to the Monte Carlo; the Monte Carlo is reweighted to make the $p_{T}$ spectra of the $B$ mesons agree better. Figure 5-44 shows the distributions of the transverse momenta for the $B^{+}$and $B^{0}$ mesons, for both Monte Carlo and sidebandsubtracted data. We find that the best fit model for the sideband subtracted data is a convolution of a Gaussian and an exponential function:

$$
f_{\text {data }}(x)=N \cdot \mathcal{G}(x \mid \mu, \sigma) \otimes \exp (x \mid \tau)
$$

For the Monte Carlo, we find that the best analytical description is given with a Gaussian and a product of an exponential and power function:

$$
f_{M C}(x)=N_{G} \cdot \mathcal{G}(x \mid \mu, \sigma)+N_{P} \cdot e^{-\lambda *(x-\delta)} *(x-\delta)^{\eta}
$$

We use the ratio of the analytical fit to the data and the analytical fit to the Monte Carlo to define the reweighting function. The reweighting functions for the $B^{+}$and $B^{0}$ spectra are shown in Figure 5-45. What appears to be a spike in these distributions is really just an artifact of our convention that we set the value of the reweighting function to be 1 in the range where there is no Monte Carlo to be reweighted.

We reweight the Monte Carlo while applying the selection cuts. This means that all the comparisons between data and Monte Carlo are done with reweighted Monte Carlo. It also means that the final ("total") efficiencies, which are used to calculate the ratios of branching fractions, are calculated with reweighted Monte Carlo. By comparing trigger efficiencies with and without Monte Carlo reweighting, we assign a systematic uncertainty due to $B$ meson $p_{T}$ spectrum simulation. 

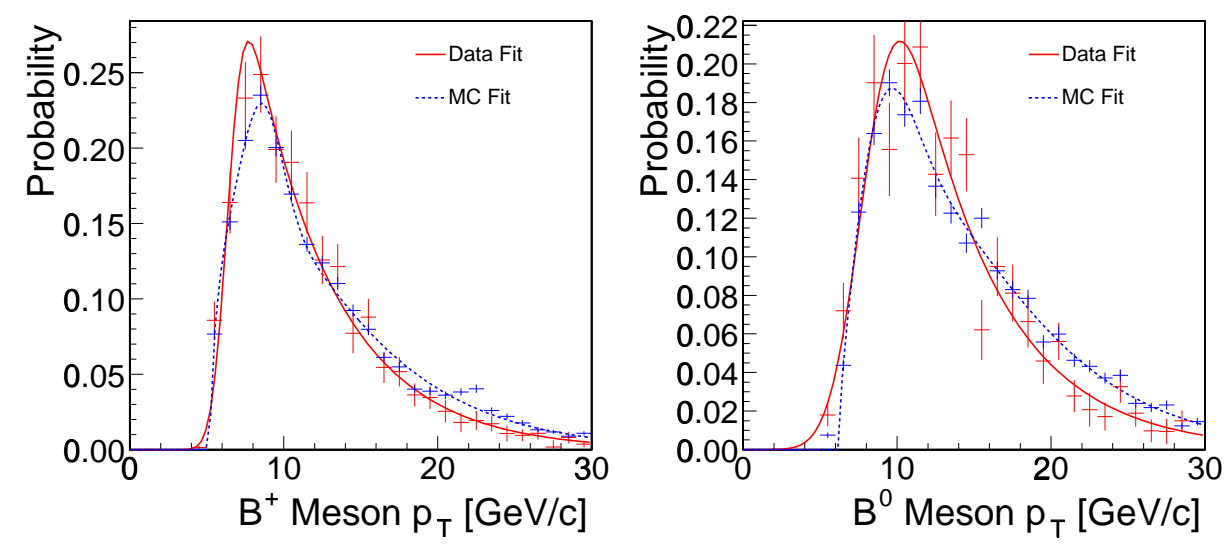

Figure 5-44: Fits of the $p_{T}$ spectra of the $B$ mesons for sideband subtracted data (solid) and Monte Carlo (dashed)
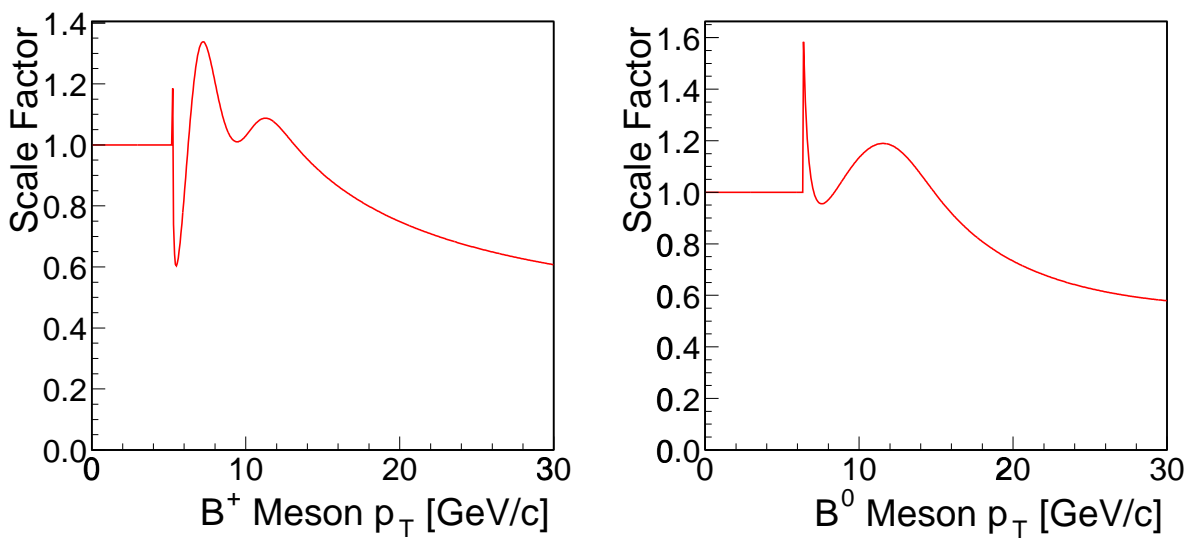

Figure 5-45: Monte Carlo reweighting functions are ratios of the fits of the individual spectra 


\subsection{Ratio of Efficiencies}

In subsection 5.1.2 we studied the success in reproducing different quantities in the Monte Carlo simulation. In this subsection, we focus on the different efficiencies that we extract from it. The detector and reconstruction efficiencies for finding different $B$ mesons are not exactly the same. We are interested in understanding the difference in efficiency for detecting $B_{s}^{0}$ decays with respect to $B^{0}$ decays as this will correct our raw numbers of detected $B_{s}^{0}$ and $B^{0}$ decays to give us ratios of branching fractions.

For more detailed understanding of the total efficiency $\left(\epsilon_{t o t}\right)$ for finding a given $B$ decay, we break up the total efficiency into two factors. These are the trigger efficiency $\left(\epsilon_{\text {trig }}\right)$ and reconstruction efficiency $\left(\epsilon_{\text {reco }}\right)$. The trigger efficiency is the efficiency with which the B_CHARM trigger path accepts a given $B$ meson decay. In terms of Monte Carlo simulation, it is defined as:

$$
\epsilon_{\text {trig }}=\frac{N_{\text {trig }}}{N_{\text {generated }}}
$$

where $N_{\text {generated }}$ is the number of generated events, and $N_{\text {trig }}$ is the number of events that pass the trigger selection. The number of generated events, $N_{\text {generated }}$ depends on the parameters (minimum $p_{T}$, maximum $|\eta|$ ) of the generation. Our choice of these parameters $\left(p_{T}>0 \mathrm{GeV} / \mathrm{c},|\eta|<6\right)$ is loose enough that the entire $B$ meson production cross section is included in the denominator. The reconstruction efficiency is the efficiency with which our reconstruction executable and analysis reconstructs $B$ meson decays that have passed the trigger selection:

$$
\epsilon_{\text {reco }}=\frac{N_{\text {reconstructed }}}{N_{\text {trig }}}
$$

where $N_{\text {reconstructed }}$ is the number of events in which the $B$ meson decay has been reconstructed by the analysis. The total efficiency is:

$$
\epsilon_{\text {tot }}=\frac{N_{\text {reconstructed }}}{N_{\text {generated }}}=\epsilon_{\text {trig }} \cdot \epsilon_{\text {reco }}
$$

As before, the dataset is broken up into run ranges. A single run represents the 
data taken in the run range. Table 5.9 shows the trigger efficiencies yielded for the different representative runs for both datasets. Tables 5.10 and shows the corresponding reconstruction efficiencies for the same runs. For visualization purposes, we plot these efficiencies as a function of represented luminosity, in Figures 5-46 and 5-47. All plots are divided by a dashed line in the middle which represents the separation point between the winter (hbot0h) and summer (hbot1i) datasets.

The variations in trigger efficiency are more pronounced in the first part of the data; after the shutdown, the trigger efficiency appears to stabilize. The reconstruction efficiencies show much more stability. However, for our measurement, it is most important that the ratio of total efficiencies does not change much with trigger conditions; if this ratio is stable, the analysis is essentially robust against any potentially poorly simulated trigger or detector effects, and we expect to have small systematic errors from the Monte Carlo simulation. The structures which are visible in individual plots in Figures 5-46 and 5-47 are consistent across all three $B$ meson decays and cancel in the ratio. The dependance of the ratio of total efficiencies for $B_{s}^{0}$ versus $B^{0}$ decays and $B^{+}$versus $B^{0}$ decays is shown in Figure 5-48. The distributions are consistent with no dependance on the trigger configuration. For evaluating the correction factor due to different total efficiencies for the different $B$ mesons, we average the efficiencies according to the luminosities that they represent:

$$
\overline{\epsilon_{t o t}}=\frac{\sum_{i} \mathcal{L}^{i} \epsilon_{t o t}^{i}}{\sum_{i} \mathcal{L}^{i}}
$$

where $\mathcal{L}^{i}$ is the luminosity of a given run range. The luminosity averaged efficiencies are listed in Table 5.11. 


\begin{tabular}{c|c|c|c} 
Run & $\epsilon\left(B_{s}\right)[\%]$ & $\epsilon\left(B^{+}\right)[\%]$ & $\epsilon\left(B^{0}\right)[\%]$ \\
\hline \hline 141508 & $0.185 \pm 0.004$ & $0.216 \pm 0.004$ & $0.206 \pm 0.004$ \\
144578 & $0.155 \pm 0.004$ & $0.185 \pm 0.004$ & $0.172 \pm 0.004$ \\
146920 & $0.176 \pm 0.004$ & $0.211 \pm 0.004$ & $0.197 \pm 0.004$ \\
150799 & $0.267 \pm 0.005$ & $0.315 \pm 0.005$ & $0.292 \pm 0.005$ \\
151513 & $0.236 \pm 0.004$ & $0.277 \pm 0.005$ & $0.258 \pm 0.005$ \\
150799 & $0.267 \pm 0.005$ & $0.315 \pm 0.005$ & $0.292 \pm 0.005$ \\
153618 & $0.268 \pm 0.005$ & $0.317 \pm 0.005$ & $0.302 \pm 0.005$ \\
\hline 160230 & $0.285 \pm 0.005$ & $0.338 \pm 0.005$ & $0.317 \pm 0.005$ \\
160823 & $0.285 \pm 0.005$ & $0.349 \pm 0.005$ & $0.323 \pm 0.005$ \\
161379 & $0.287 \pm 0.005$ & $0.325 \pm 0.005$ & $0.310 \pm 0.005$ \\
161678 & $0.293 \pm 0.005$ & $0.342 \pm 0.005$ & $0.325 \pm 0.005$ \\
162423 & $0.285 \pm 0.005$ & $0.331 \pm 0.005$ & $0.306 \pm 0.005$ \\
162498 & $0.282 \pm 0.005$ & $0.337 \pm 0.005$ & $0.310 \pm 0.005$ \\
162857 & $0.280 \pm 0.005$ & $0.338 \pm 0.005$ & $0.313 \pm 0.005$ \\
163064 & $0.283 \pm 0.005$ & $0.338 \pm 0.005$ & $0.314 \pm 0.005$
\end{tabular}

Table 5.9: Trigger efficiencies from realistic Monte Carlo for the different $B$ decay channels and the different representative runs.

\begin{tabular}{c|c|c|c} 
Run & $\epsilon\left(B_{s}\right)$ & $\epsilon\left(B^{+}\right)$ & $\epsilon\left(B^{0}\right)$ \\
\hline \hline 141508 & $0.386 \pm 0.010$ & $0.386 \pm 0.010$ & $0.386 \pm 0.010$ \\
144578 & $0.337 \pm 0.011$ & $0.337 \pm 0.011$ & $0.337 \pm 0.011$ \\
146920 & $0.357 \pm 0.010$ & $0.357 \pm 0.010$ & $0.357 \pm 0.010$ \\
150799 & $0.340 \pm 0.008$ & $0.340 \pm 0.008$ & $0.340 \pm 0.008$ \\
151513 & $0.335 \pm 0.009$ & $0.335 \pm 0.009$ & $0.335 \pm 0.009$ \\
152747 & $0.345 \pm 0.009$ & $0.345 \pm 0.009$ & $0.345 \pm 0.009$ \\
\hline 160230 & $0.357 \pm 0.008$ & $0.357 \pm 0.008$ & $0.357 \pm 0.008$ \\
160823 & $0.354 \pm 0.008$ & $0.354 \pm 0.008$ & $0.354 \pm 0.008$ \\
161379 & $0.323 \pm 0.008$ & $0.323 \pm 0.008$ & $0.323 \pm 0.008$ \\
161678 & $0.330 \pm 0.008$ & $0.330 \pm 0.008$ & $0.330 \pm 0.008$ \\
162423 & $0.376 \pm 0.008$ & $0.376 \pm 0.008$ & $0.376 \pm 0.008$ \\
162498 & $0.364 \pm 0.008$ & $0.364 \pm 0.008$ & $0.364 \pm 0.008$ \\
162857 & $0.351 \pm 0.008$ & $0.351 \pm 0.008$ & $0.351 \pm 0.008$ \\
163064 & $0.346 \pm 0.008$ & $0.346 \pm 0.008$ & $0.346 \pm 0.008$
\end{tabular}

Table 5.10: Reconstruction efficiencies from realistic Monte Carlo for the different $B$ decay channels and the different representative runs. 


\begin{tabular}{c|c|c|c} 
hbot0h & $B_{s}^{0}$ & $B^{+}$ & $B^{0}$ \\
\hline \hline Trig. (\%) & $0.2404 \pm 0.0026$ & $0.2842 \pm 0.0029$ & $0.2685 \pm 0.0028$ \\
Recon. & $0.3498 \pm 0.0049$ & $0.3912 \pm 0.0046$ & $0.2910 \pm 0.0044$ \\
\hline Total (\%) & $0.0840 \pm 0.0016$ & $0.1114 \pm 0.0018$ & $0.0782 \pm 0.0015$
\end{tabular}

\begin{tabular}{c|c|c|c} 
hbot1i & $B_{s}^{0}$ & $B^{+}$ & $B^{0}$ \\
\hline \hline Trig. (\%) & $0.2850 \pm 0.0017$ & $0.3371 \pm 0.0019$ & $0.3146 \pm 0.0018$ \\
Recon. & $0.3501 \pm 0.0029$ & $0.3923 \pm 0.0027$ & $0.3020 \pm 0.0026$ \\
\hline Total (\%) & $0.0997 \pm 0.0010$ & $0.1323 \pm 0.0012$ & $0.0950 \pm 0.0010$
\end{tabular}

\begin{tabular}{c|c|c|c} 
total & $B_{s}^{0}$ & $B^{+}$ & $B^{0}$ \\
\hline \hline Trig. (\%) & $0.2607 \pm 0.0016$ & $0.3083 \pm 0.0018$ & $0.2895 \pm 0.0017$ \\
Recon. & $0.3499 \pm 0.0030$ & $0.3917 \pm 0.0028$ & $0.2960 \pm 0.0027$ \\
\hline Total (\%) & $0.0912 \pm 0.0010$ & $0.1209 \pm 0.0011$ & $0.0858 \pm 0.0009$
\end{tabular}

Table 5.11: Luminosity averaged efficiencies from realistic Monte Carlo for the different $B$ decay channels. The top table lists efficiencies for the winter dataset. The middle and bottom tables list efficiencies for the summer and combined dataset. 

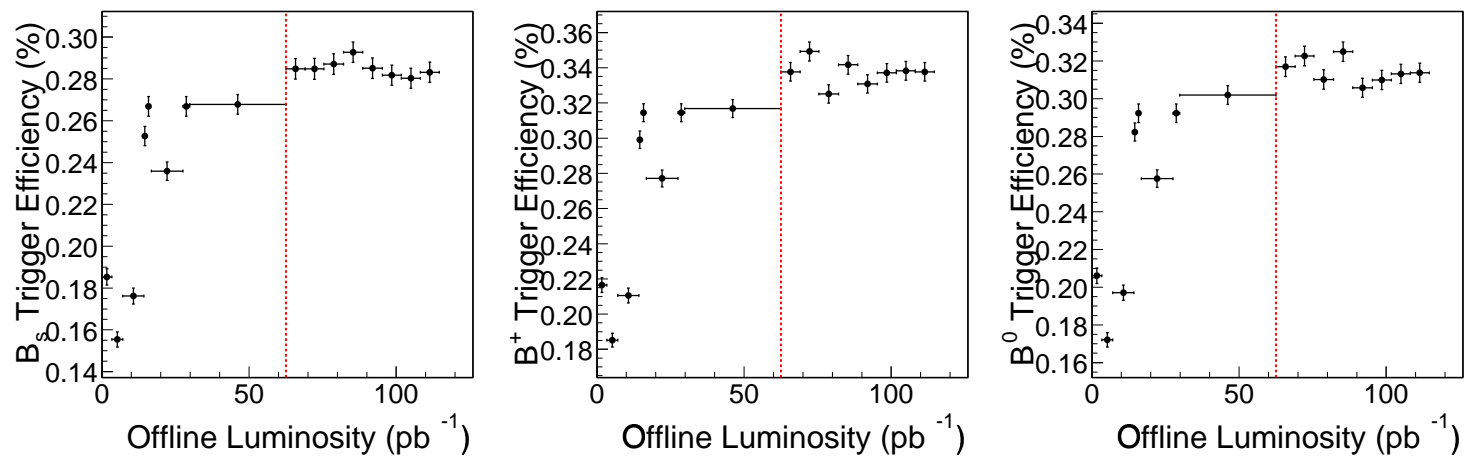

Figure 5-46: Variation of trigger efficiencies as a function of run conditions. The leftmost plot shows the trigger efficiency variations for the $B_{s}^{0}$, the middle for the $B^{+}$ and the rightmost plot for the $B^{0}$. The luminosity covered by the representative run is represented by the $\mathrm{x}$ error span on a given point.
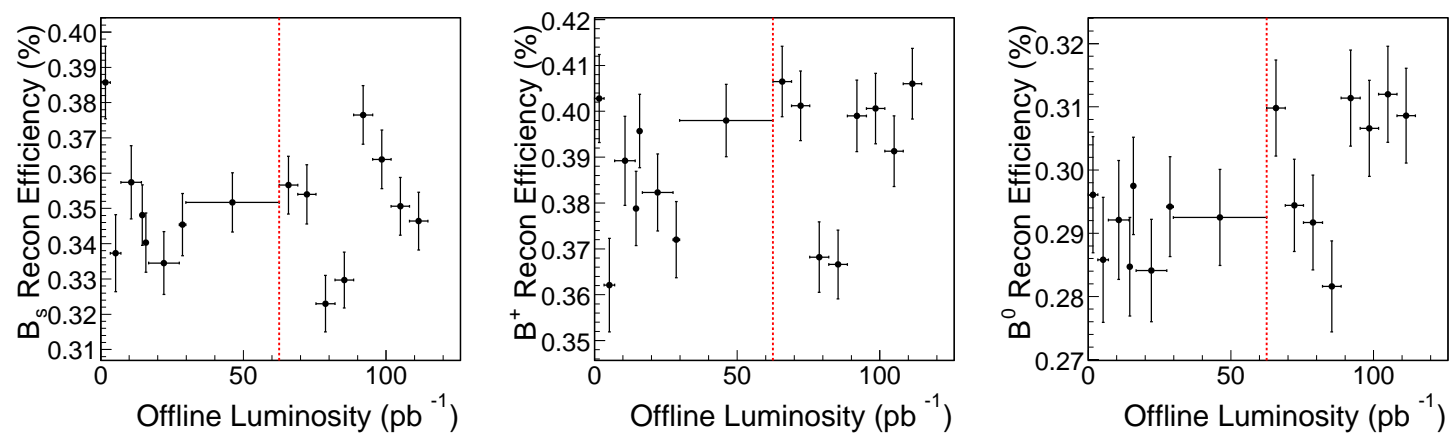

Figure 5-47: Variation of reconstruction efficiencies as a function of run conditions. The leftmost plot shows the reconstruction efficiency variations for the $B_{s}^{0}$, the middle for the $B^{+}$and the rightmost plot for the $B^{0}$. The luminosity covered by the representative run is denoted by the $\mathrm{x}$ error span on a given point. 

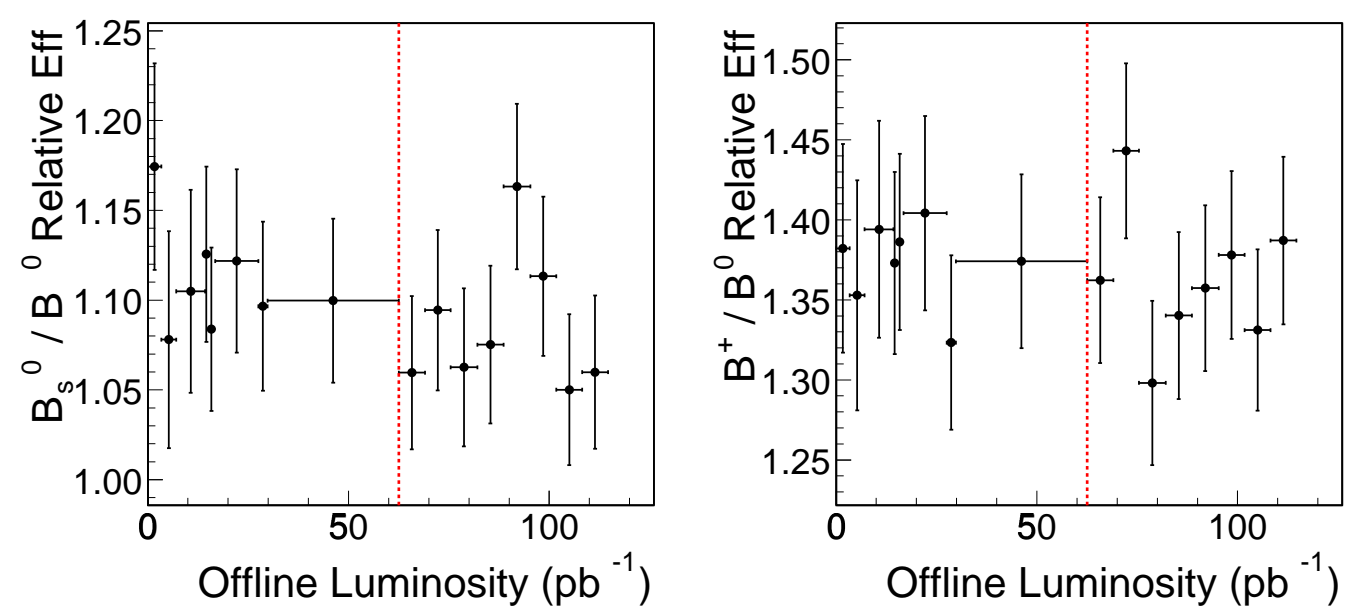

Figure 5-48: Variation of relative total efficiencies (trigger $\times$ reconstruction) for different run conditions. The left plot shows the ratio for $B_{s}^{0}$ mesons relative to $B^{0}$ mesons, and the right plot shows the ratio for $B^{+}$mesons relative to $B^{0}$ mesons. Neither distribution shows statistically significant variation with respect to run conditions. 


\subsection{Ratio of Branching Fractions}

Having measured the rates of different $B$ mesons in our data, and having estimated the efficiencies of the trigger and analysis software, we deduce the ratios of branching fractions for the winter, summer and combined datasets. We apply the formula:

$$
\frac{f_{s}}{f_{d}} \cdot \frac{B r\left(B_{s}^{0} \rightarrow D_{s} \pi\right)}{B r\left(B^{0} \rightarrow D^{-} \pi\right)}=\frac{N\left(B_{s}^{0}\right)}{N\left(B^{0}\right)} \cdot \frac{\epsilon\left(B^{0}\right)}{\epsilon\left(B_{s}^{0}\right)} \cdot \frac{B r\left(D^{+} \rightarrow K \pi \pi\right)}{B r\left(D_{s} \rightarrow \phi \pi, \phi \rightarrow K K\right)}
$$

using values of luminosity averaged efficiencies as found in Table 5.11 and rates of $B$ mesons as described in Section 5.2.6. We substitute $B_{s}^{0}$ for $B^{+}$in this equation assuming $f_{u} / f_{d}=1$ and using the corresponding $D_{0}$ branching ratio to obtain the ratio of branching fractions for the $B^{+}$mesons, too. For the winter dataset, we obtain:

$$
\begin{array}{r}
\frac{f_{s}}{f_{d}} \cdot \frac{B r\left(B_{s}^{0} \rightarrow D_{s} \pi\right)}{B r\left(B^{0} \rightarrow D^{+} \pi\right)}=0.353 \pm 0.066(\text { stat }) \pm 0.091(B R) \\
\frac{B r\left(B^{+} \rightarrow D^{0} \pi\right)}{B r\left(B^{0} \rightarrow D^{+} \pi\right)}=2.18 \pm 0.16(\text { stat }) \pm 0.15(B R)
\end{array}
$$

For the summer dataset, we obtain:

$$
\begin{array}{r}
\frac{f_{s}}{f_{d}} \cdot \frac{B r\left(B_{s}^{0} \rightarrow D_{s} \pi\right)}{B r\left(B^{0} \rightarrow D^{+} \pi\right)}=0.299 \pm 0.065(\text { stat }) \pm 0.077(B R) \\
\quad \frac{B r\left(B^{+} \rightarrow D^{0} \pi\right)}{B r\left(B^{0} \rightarrow D^{+} \pi\right)}=1.98 \pm 0.14(\text { stat }) \pm 0.14(B R)
\end{array}
$$

The two measurements are consistent within statistical errors, and we can combine the two datasets. For the combined dataset, we obtain:

$$
\begin{array}{r}
\frac{f_{s}}{f_{d}} \cdot \frac{B r\left(B_{s}^{0} \rightarrow D_{s} \pi\right)}{B r\left(B^{0} \rightarrow D^{+} \pi\right)}=0.325 \pm 0.046(\text { stat }) \pm 0.084(B R) \\
\frac{B r\left(B^{+} \rightarrow D^{0} \pi\right)}{B r\left(B^{0} \rightarrow D^{+} \pi\right)}=2.07 \pm 0.11(\text { stat }) \pm 0.14(B R)
\end{array}
$$

So far, we quote measurement results for both the ratio of $B_{s}^{0}$ and $B^{+}$mesons relative to $B^{0}$ mesons. The analysis of systematic uncertainties will focus on the ratio of branching fractions of $B_{s}^{0}$ decays relative to $B^{0}$ decays and use $B^{+}$decays as a 
high-statistics sample for cross-checks. In order to correctly estimate the systematic uncertainty for $B^{+}$decays relative to $B^{0}$ decays, an additional study is needed to estimate the systematic effects of having different numbers of tracks in the final state (three for $B^{+}$decays versus four for $B^{0}$ decays). 


\subsection{Systematic Uncertainties}

The previous sections dealt with the procedures of measuring the ratio of branching fractions. In this section, we estimate the systematic uncertainties of the measurement. Our measurement focuses on the ratio of branching fractions of two kinematically very similar decays: $B_{s}^{0} \rightarrow D_{s}^{-} \pi^{+}$and $B^{0} \rightarrow D^{-} \pi^{+}$. We therefore expect that most of the systematic uncertainties for the individual channels cancel to first order in the ratio, and expect a small systematic uncertainty on the measurement of the ratio. In order to determine the sources of systematic uncertainties, we have to consider what effects are different between the $B_{s}^{0}$ and $B^{0}$ decays.

The first effect that we investigate is the input $B$ meson production $p_{T}$ spectrum used in Monte Carlo generation. Due to different meson masses, the generated momentum spectra of the $B_{s}^{0}$ and $B^{0}$ are slightly different. A discrepancy between the real and simulated spectrum will introduce a systematic shift of the efficiencies. The $B_{s}^{0}$ and $B^{0}$ mesons and particularly the $D_{s}^{-}$and $D^{-}$mesons have different lifetimes which cause differences in the trigger acceptance. We also study limitations of the detector simulation program, uncertainties on the number of reconstructed $B$ mesons returned from the fit, and uncertainties introduced by estimating cut efficiencies as potential sources of systematic uncertainties.

\subsubsection{The $B$ Meson $p_{T}$ Spectrum}

A discrepancy was noticed between the $B$ meson $p_{T}$ spectrum observed in data to that obtained from Monte Carlo simulation, as shown in Figure 5-44. To resolve this discrepancy, we introduce a reweighting procedure to correct the $B$ meson $p_{T}$ spectrum. To obtain the systematic effect from imperfections in our $p_{T}$ spectrum, we compare ratios of total efficiencies when the $p_{T}$ spectra are scaled to the case when they are not. From the difference in efficiencies, we assign a $\pm 1.5 \%$ systematic uncertainty to $B$ meson $p_{T}$ spectrum effects. 


\subsection{2 $B$ and $D$ Lifetimes}

We use the world average [10] measured values of $B$ and $D$ lifetimes as input to the $B$ meson decay simulation. Using an incorrect lifetime results in incorrect trigger or reconstruction efficiencies, because the two-track trigger is based on track displacement. This causes the efficiencies to depend on the lifetimes of the decaying particles. In order to estimate the size of this effect, we vary the $B$ and $D$ meson lifetimes by their uncertainties, as listed in Table 5.12. We then reweight the Monte Carlo to reflect the new lifetime, and calculate the corrected efficiency. Comparing efficiencies before and after reweighting the Monte Carlo sample gives a very precise estimate of the effect of different lifetimes as it uses the exact same Monte Carlo events. We list the relative changes in efficiencies when rescaling the Monte Carlo in Table 5.13. These are then propagated as uncorrelated into our estimates of the total systematic uncertainties.

It is interesting to compare the relative uncertainties in Table 5.12 to the systematic uncertainties of Table 5.13. A rough comparison is possible between the two four-track states $\left(B_{s}^{0}\right.$ and $\left.B^{0}\right)$. The relative uncertainty on the $B_{s}^{0}$ lifetime is 3.8 times larger than that of the $B^{0}$ lifetime. The corresponding systematic uncertainty from the $B_{s}^{0}$ lifetime is 5 times larger than that of the $B^{0}$ systematic uncertainty. The $D_{s}^{-}$meson and $D^{-}$meson have considerably different lifetimes which also modify the trigger efficiencies. Taking that into account, the systematic uncertainties from $B_{s}^{0}$ and $B^{0}$ lifetimes are in rough agreement with each other.

\begin{tabular}{c|c} 
Meson & Lifetime \\
\hline \hline$B_{s}^{0}$ & $1.461 \pm 0.057 \mathrm{ps}$ \\
$B^{+}$ & $1.674 \pm 0.018 \mathrm{ps}$ \\
$B^{0}$ & $1.542 \pm 0.016 \mathrm{ps}$ \\
\hline$D_{s}^{+}$ & $490 \pm 9 \mathrm{fs}$ \\
$D^{0}$ & $411.7 \pm 2.7 \mathrm{fs}$ \\
$D^{+}$ & $1051 \pm 13 \mathrm{fs}$
\end{tabular}

Table 5.12: $B$ and $D$ meson lifetimes and errors used to estimate systematic effects by reweighting the Monte Carlo 


\begin{tabular}{l|c} 
Effect & Syst. Uncertainty \\
\hline \hline$B_{s}^{0}$ lifetime & $\pm 2.00 \%$ \\
$D_{s}^{+}$lifetime & $\pm 0.20 \%$ \\
$B^{+}$lifetime & $\pm 0.20 \%$ \\
$D^{0}$ lifetime & $\pm 0.04 \%$ \\
$B^{0}$ lifetime & $\pm 0.40 \%$ \\
$D^{+}$lifetime & $\pm 0.15 \%$
\end{tabular}

Table 5.13: Table of systematic uncertainties due to uncertainties on meson lifetimes

\subsubsection{XFT Simulation}

There are two effects which are not well simulated in the Monte Carlo which affect the measurement of branching fractions. The first effect has been observed when measuring the relative branching fraction of $D^{0} \rightarrow \pi^{+} \pi^{-}$to $D^{0} \rightarrow K^{-} \pi^{+}$.[54]. It has been observed that the relative efficiency of XFT triggering on kaon tracks is lower than that of pion tracks. This is due to the different energy deposition in the COT wires from kaons, which results in a different XFT hit finding efficiency and consequently a different track finding efficiency. This effect is not reproduced in the Monte Carlo. The other effect of concern is that XFT pattern recognition efficiency drops for high $(\sim 5 \mathrm{~mm})$ displacements of the beamline from the center of the detector, which have been observed in all our data.

To study the effect of different efficiencies when triggering on kaon and pion tracks, we simulate the effect on our Monte Carlo sample. The $D^{0}$ branching fraction analysis has found that the efficiency drop can be simulated well in Monte Carlo by introducing a $6 \%$ inefficiency when triggering on a kaon in the event, regardless of the kaon $p_{T}$ [54]. When we follow the same procedure in our Monte Carlo samples, we see a $2 \%$ change in the relative efficiency of the $B_{s}^{0}$ trigger efficiency to that of the $B^{0}$. We do not correct for this effect. Instead, we assign a $\pm 1 \%$ systematic uncertainty for it.

To check for the effect of XFT efficiency drop due to large displacements of the beamline from the center of the detector, we study the $\varphi_{0}$ distribution of reconstructed $B$ mesons. Due to the large displacement, the XFT pattern recognition has different efficiencies depending on the $\varphi_{0}$ of the track being reconstructed. This effect manifests 
itself in a discrepancy of shape between the $\varphi_{0}$ distributions of the data and Monte Carlo for reconstructed $B$ mesons. Figures 5-14 and 5-15 show a comparison between data and Monte Carlo for the $\varphi_{0}$ of the reconstructed $B^{+}$and $B^{0}$ mesons. No major discrepancies are found in this distribution, and no additional systematic error is assigned. The total systematic uncertainty due to XFT simulation is estimated to be $\pm 1 \%$.

\subsubsection{Fitting}

Our fitting method uses templates to describe different background shapes. This means that certain parameters are fixed in the fit to the data. These values are extracted from Monte Carlo and have corresponding errors on them, as listed in Tables 5.2 through 5.4. We estimate one part of our systematic uncertainty due to fitting by varying the values of the parameters in the templated fit. The other part of our estimate of the systematic uncertainty comes from increasing the range of the fit, to include more combinatorial background.

In all three decay modes studied, including more data on the high mass side, but keeping all the template shapes fixed makes little or no change on the number of $B$ mesons in the event, but the fit quality is not very good (fit probability below $1 \%)$. We expand the test by fixing the combinatorial background parameters from the high mass sideband and letting the slope and fraction of the " $B$-continuum" background float in the fit. In case our fitting model omits an additional continuumtype background, the floating shape in the template adjusts itself to compensate. The fits for these tests are depicted in Figure 5-49. We see an 6-8\% increase in the number of $B^{0}, B^{+}$meson candidates returned by the fit when this method is applied so we assign these values as systematic errors due to the limitations of the fitting model. The effect seen in the $B_{s}^{0}$ fit is much smaller, because this channel has much less background due to the $\phi$ invariant mass requirement. The final estimates of the systematic uncertainty due to all the combined fitting effects are listed in Tables 5.15 through 5.16 . 

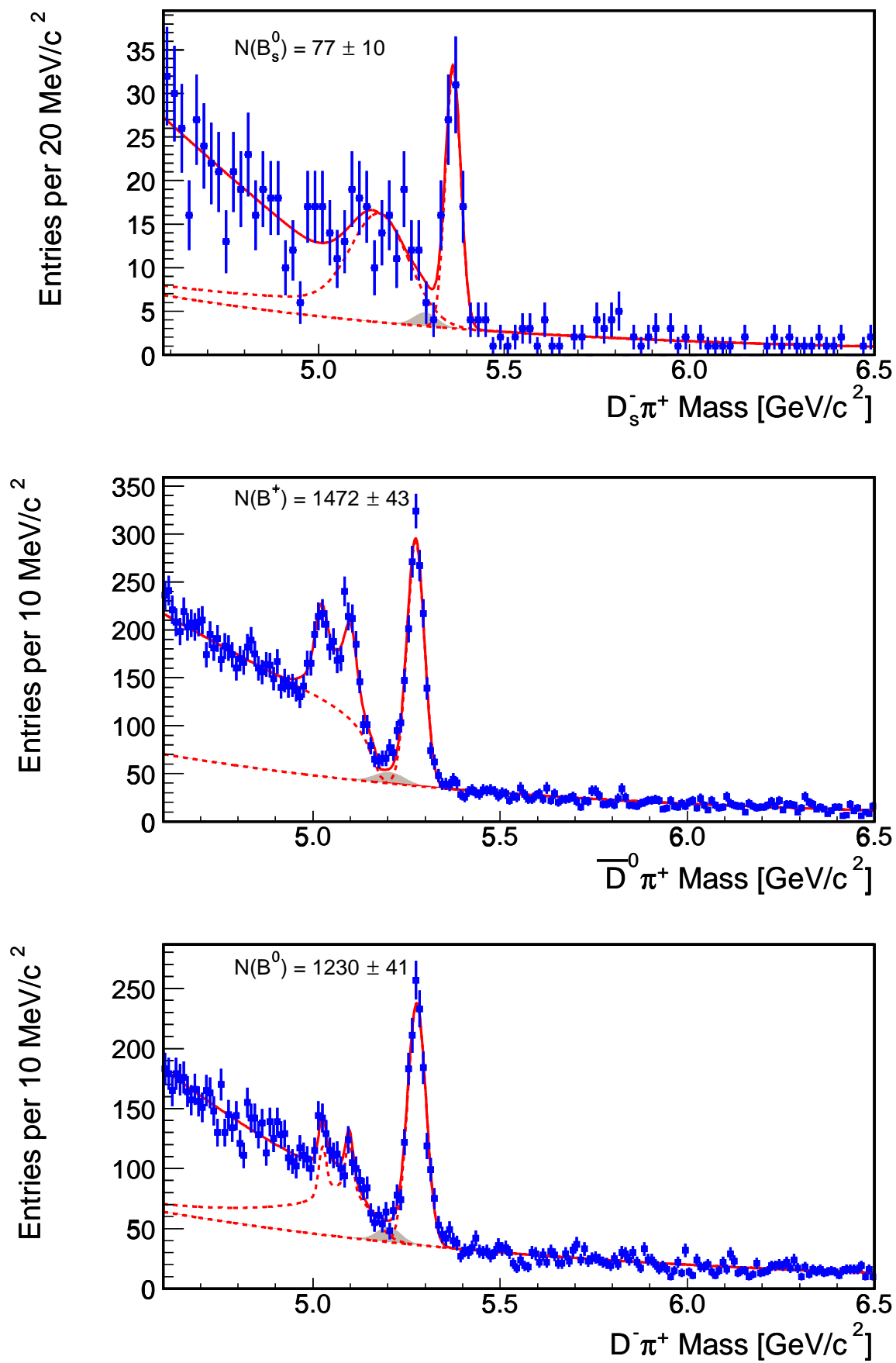

Figure 5-49: Fit results for extended fit range, as part of estimating the systematic uncertainty due to limitations of the fitting model. 


\begin{tabular}{c|l|c} 
Parm & Effect & Syst. Uncertainty [\%] \\
\hline \hline$f_{C}$ & Cabbibo supp. fraction & $+0.458 /-0.488$ \\
$\delta_{C}$ & Cabbibo mass diff $\left[\mathrm{GeV} / \mathrm{c}^{2}\right]$ & $+0.711 /-0.857$ \\
$\sigma_{C}$ & Cabbibo supp. width $\left[\mathrm{GeV} / \mathrm{c}^{2}\right]$ & $+0.439 /-0.259$ \\
$f$ & spike fraction & $+0.118 /-0.324$ \\
$\sigma_{B}$ & main body width $\left[\mathrm{GeV} / \mathrm{c}^{2}\right]$ & $+0.089 /-0.147$ \\
$\sigma_{H}$ & spike width $\left[\mathrm{GeV} / \mathrm{c}^{2}\right]$ & $+0.087 /-0.392$ \\
$N_{C} / N_{H}$ & continuum fraction & $+0.449 /-0.418$ \\
$s$ & slope $\left[1 / \mathrm{GeV} / \mathrm{c}^{2}\right]$ & $+0.206 /-0.209$ \\
$\tau$ & turnoff lifetime $\left[1 / \mathrm{GeV} / \mathrm{c}^{2}\right]$ & $+0.352 /-0.169$ \\
\hline & extended fit range & $+4.812 /-4.812$ \\
\hline & Total & $+4.946 /-4.972$
\end{tabular}

Table 5.14: Table of systematic uncertainties on the number of $B^{+}$events as returned by the templated fitter.

\begin{tabular}{c|l|c} 
Parm & Effect & Syst. Uncertainty [\%] \\
\hline \hline$f_{C}$ & Cabbibo suppressed fraction & $+0.476 /-0.637$ \\
$\delta_{C}$ & Cabbibo mass diff $\left[\mathrm{GeV} / \mathrm{c}^{2}\right]$ & $+1.119 /-1.182$ \\
$\sigma_{C}$ & Cabbibo supp. width $\left[\mathrm{GeV} / \mathrm{c}^{2}\right]$ & $+0.087 /-0.157$ \\
$\lambda$ & refl. "lifetime" $\left[1 / \mathrm{GeV} / \mathrm{c}^{2}\right]$ & $+0.754 /-3.060$ \\
$f$ & reflection gaus. frac & $+2.851 /-1.302$ \\
$\sigma$ & reflection width $\left[\mathrm{GeV} / \mathrm{c}^{2}\right]$ & $+0.500 / 0.000$ \\
$N_{R} / N_{L}$ & "B continuum" frac & $+2.681 /-1.546$ \\
$L$ & "B continuum" cutoff $\left[\mathrm{GeV} / \mathrm{c}^{2}\right]$ & $+1.198 /-3.553$ \\
\hline & extended fit range & $+1.318 /-1.318$ \\
\hline & Total & $+4.559 /-5.444$
\end{tabular}

Table 5.15: Table of systematic uncertainties on the number of $B_{s}^{0}$ events as returned by the templated fitter. 


\begin{tabular}{c|l|c} 
Parm & Effect & Syst. Uncertainty [\%] \\
\hline \hline$f_{C}$ & Cabbibo suppressed fraction & $+1.010 /-1.070$ \\
$\delta_{C}$ & Cabbibo mass diff $\left[\mathrm{GeV} / \mathrm{c}^{2}\right]$ & $+0.534 /-0.538$ \\
$\sigma_{C}$ & Cabbibo supp. width $\left[\mathrm{GeV} / \mathrm{c}^{2}\right]$ & $+2.536 / 0.000$ \\
$\lambda$ & reflect. "lifetime" [GeV/c $\left.\mathrm{c}^{2}\right]$ & $+0.282 /-0.346$ \\
$f_{G}$ & reflection Gaus. frac. & $+0.876 /-0.928$ \\
$\sigma_{R}$ & reflection width [GeV/c $\left.\mathrm{c}^{2}\right]$ & $+0.119 /-0.127$ \\
$f_{H}$ & spike frac & $+0.740 /-0.775$ \\
$\delta$ & spike separation $\left[\mathrm{GeV} / \mathrm{c}^{2}\right]$ & $+0.178 /-0.297$ \\
$\sigma_{H}$ & spike width $\left[\mathrm{GeV} / \mathrm{c}^{2}\right]$ & $+0.547 / 0.000$ \\
$N_{R} / N_{L}$ & continuum frac & $+0.418 /-0.375$ \\
$\chi$ & continuum cutoff $\left[\mathrm{GeV} / \mathrm{c}^{2}\right]$ & $+0.070 /-0.066$ \\
\hline & extended fit range & $+6.658 /-6.658$ \\
\hline & Total & $+7.348 /-6.899$
\end{tabular}

Table 5.16: Table of systematic uncertainties on the number of $B^{0}$ events as returned by the templated fitter. 


\subsubsection{Cut Efficiencies}

In Section 5.3, we explain our method for doing sideband subtraction to compare distributions in data and Monte Carlo. We apply a similar method to evaluate cut efficiencies and compare them to Monte Carlo predictions on the high-statistics samples $B^{+}, B^{0}$. This provides us with an estimate of how well the relative cut efficiencies are modeled for the low-statistics sample of $B_{s}^{0}$ decays.

We chose to compare (N-1)-type cut efficiencies, which means we study the efficiency of a single cut given that the other (N-1) cuts of the selection are applied. We define the number of events that pass the other $(\mathrm{N}-1)$ cuts for the signal region as $N_{s}$ and for the sideband as $N_{b}$. The number of events that pass all $\mathrm{N}$ cuts is $n_{s}$ for the signal region and $n_{b}$ for the sideband region. Then we are interested in the ratio:

$$
p_{c u t}^{d a t a}=\frac{n_{s}-n_{b}}{N_{s}-N_{b}}
$$

which can be directly compared to the (N-1) cut efficiency that we measure for the same cut in Monte Carlo. If we further define the probabilities for passing cut $\mathrm{N}$ given that the other $(\mathrm{N}-1)$ cuts are passed in the signal region as $p_{s}=n_{s} / N_{s}$ and for the background region $p_{b}=n_{b} / N_{b}$ then the statistical error on $p_{c u t}^{\text {data }}$ can be derived using propagation of binomial errors:

$$
\sigma\left(p_{c u t}^{d a t a}\right)=\frac{\sqrt{N_{s} p_{s}\left(1-p_{s}\right)+N_{b} p_{b}\left(1-p_{b}\right)}}{N_{s}-N_{b}}
$$

In reality, we are interested in comparing ratios of cut efficiencies. So we in fact compare the ratios $p_{c u t}^{\text {data }}\left(B^{+}\right) / p_{c u t}^{\text {data }}\left(B^{0}\right)$ and $p_{c u t}^{M C}\left(B^{+}\right) / p_{c u t}^{M C}\left(B^{0}\right)$. This tells us how much the ratio of cut efficiencies for the different $B$ mesons is different between data and Monte Carlo, and therefore gives us an estimate of the potential systematic discrepancy due to incorrect modeling of distributions in the Monte Carlo.

Table 5.17 shows a comparison of ratios of cut efficiencies in $B^{+}$and $B^{0}$ decays between data and Monte Carlo. The relevant quantity is $R$, the ratio of relative cut 


\begin{tabular}{l|c|c|c} 
Cut & $1-R(\%)$ & $\sigma(1-R)(\%)$ & Significance \\
\hline \hline$\chi^{2}(D)$ & -0.28 & 0.33 & 0.828 \\
$\chi^{2}(B)$ & -3.82 & 2.43 & 1.574 \\
$p_{T}(D)$ & -0.41 & 1.05 & 0.391 \\
$p_{T}(B)$ & 0.01 & 0.10 & 0.085 \\
$L_{x y}(D)$ & -0.33 & 1.57 & 0.214 \\
$L_{x y}(B)$ & 0.64 & 2.47 & 0.260 \\
$L_{x y}(B \rightarrow D)$ & -2.07 & 1.43 & 1.451 \\
$\Delta R\left(D, \pi_{B}\right)$ & -0.67 & 0.88 & 0.756 \\
$\left|d_{0}(B)\right|$ & 9.60 & 3.53 & 2.718 \\
$p_{T}\left(\pi_{B}\right)$ & -0.07 & 2.67 & 0.027
\end{tabular}

Table 5.17: Comparison of the ratio of relative (N-1) type efficiencies $(R)$ as measured in data and Monte Carlo for different cut variables

efficiencies in data and Monte Carlo:

$$
R=\frac{p_{c u t}^{\text {data }}\left(B^{+}\right) / p_{c u t}^{\text {data }}\left(B^{0}\right)}{p_{c u t}^{M C}\left(B^{+}\right) / p_{c u t}^{M C}\left(B^{0}\right)}
$$

or actually how much it differs from 1 , which is to say $R-1$. We assign a systematic uncertainty due to a relative cut efficiency mismeasurement in case $R-1$ is at least $2 \sigma$ different from 0 . We find only one cut which meets this criteria, and it is the $|d 0(B)|$ cut. We assign a $\pm 5 \%$ systematic uncertainty due to the discrepancy in relative cut efficiency in data and Monte Carlo.

\subsubsection{The $\phi^{0}$ Invariant Mass Requirement}

In reconstructing the $B_{s}^{0} \rightarrow D_{s}^{-} \pi^{+}, D_{s}^{-} \rightarrow \phi^{0} \pi^{-}$decays, we require that the invariant mass of the $\phi^{0}$ in the $\phi^{0} \rightarrow K^{+} K^{-}$decay be between $1.013 \mathrm{GeV} / \mathrm{c}^{2}$ and $1.028 \mathrm{GeV} / \mathrm{c}^{2}$. The world average [10] mass of the $\phi^{0}$ is $1019.417 \pm 0.014 \mathrm{MeV} / \mathrm{c}^{2}$. If the mass spectrum of $\phi^{0} \rightarrow K^{+} K^{-}$decays in data does not agree with the distribution we get from the realistic Monte Carlo simulation, the total efficiency is incorrect and this directly biases the $B_{s}^{0}$ branching ratio measurement, as this cut is not applied to the $B^{0}$ or $B^{+}$decay reconstructions. We have several cross-checks that we can use to determine if this is a significant effect in our analysis. 
The $\phi^{0} \rightarrow K^{+} K^{-}$invariant mass distribution is modeled with a Breit-Wigner function convoluted with the detector resolution function, which is modeled with a Gaussian distribution. The world average value for the width of the Breit-Wigner component is $4.26 \pm 0.03 \mathrm{MeV} / \mathrm{c}^{2}$ [10]. Figure 5-50 shows fits of Monte Carlo and data distributions to such a convolution. The background is modeled with a linear function. From the integral of the Breit-Wigner convolution in the cut region, we determine the absolute cut efficiency. Because both the data and Monte Carlo distributions are described well with the fit functions, we can use the integral of the fit function to estimate the cut efficiency. In the Monte Carlo, we find that this efficiency is 81.6 \%. As a crosscheck in data, we use the $\phi$ mass distribution from $B_{s}^{0} \rightarrow J / \psi \phi^{0}$ decays [55], because these decays have very similar kinematic properties. We find that the

cut efficiency in the fit to the data is $80.5 \%$. The detector resolution of the $\phi^{0}$ peak in our Monte Carlo simulation is $1.3 \pm 0.2 \mathrm{MeV} / \mathrm{c}^{2}$, and the fit to the $\phi^{0}$ invariant mass distribution of $B_{s}^{0} \rightarrow J / \psi \phi^{0}$ decays returns a width of $1.8 \pm 0.5 \mathrm{MeV} / \mathrm{c}^{2}$.

Similar $\phi^{0} \rightarrow K^{+} K^{-}$decays have been studied independently using Time of Flight particle identification [56]. These studies find that with low- $p_{T}$ kaons $\left(p_{T}<\right.$ $1.5 \mathrm{GeV} / \mathrm{c}$ ), the Gaussian contribution to the $\phi^{0}$ width is $1.37 \pm 0.26 \mathrm{MeV} / \mathrm{c}^{2}$. This is consistent with findings in both data and Monte Carlo. If we use the results of this independent fit to estimate the efficiency of the $\phi^{0}$ mass cut, we find an efficiency of $79.4 \%$. We conclude that the variation of the cut efficiency between data and Monte Carlo are at the $2 \%$ level, and assign a $\pm 1 \%$ systematic uncertainty due to the efficiency of the $\phi^{0}$ mass cut.

\subsubsection{Summary of Systematic Uncertainties}

The above systematic uncertainties are added in quadrature and propagated into the branching fractions to obtain the total systematic uncertainties for the measurement, as listed in Table 5.18. The systematic uncertainty is currently dominated by limitations of the fitting method, in particular for the $B^{0} \rightarrow D^{-} \pi^{+}$background. Further studies of the background shape with more generic $B$ Monte Carlo can improve this systematic uncertainty. 

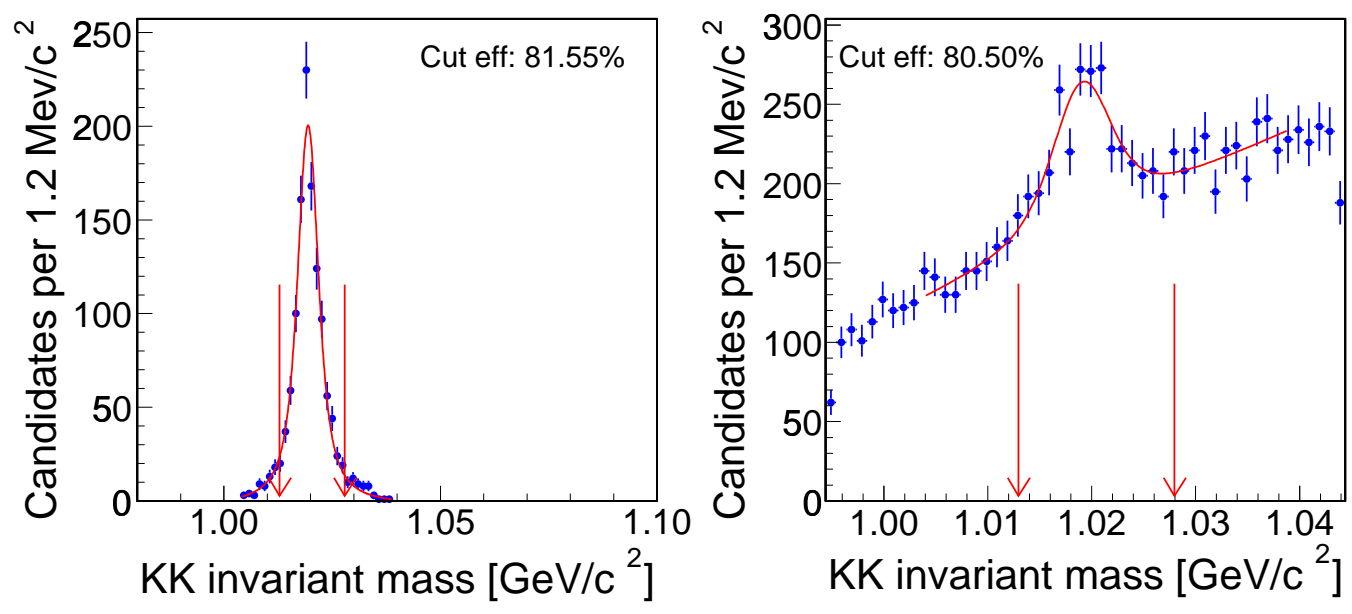

Figure 5-50: Comparison of $\phi^{0}$ mass cut efficiency in Monte Carlo and data. Both cuts are determined from the integral of the corresponding fits. Both data and Monte Carlo are fit to a Breit Wigner convoluted with the detector resolution function. On the left, the Monte Carlo shows a slightly higher cut efficiency than what is depicted on the right, in $B_{s} \rightarrow J / \psi \phi$ data. The difference is at the $1 \%$ level.

\begin{tabular}{l|c} 
Effect & Syst. Uncertainty \\
\hline \hline$B p_{T}$ spectrum & $\pm 1.5 \%$ \\
XFT simulation & $\pm 1.0 \%$ \\
$\phi^{0}$ mass cut & $\pm 1.0 \%$ \\
cut efficiencies & $\pm 5.0 \%$ \\
\hline$B_{s}^{0}$ lifetime & $\pm 2.0 \%$ \\
$D_{s}^{+}$lifetime & $\pm 0.2 \%$ \\
$B^{0}$ lifetime & $\pm 0.4 \%$ \\
$D^{+}$lifetime & $\pm 0.2 \%$ \\
\hline$B_{s}^{0}$ fitting & $\pm 5.0 \%$ \\
$B^{0}$ fitting & $\pm 7.0 \%$ \\
\hline Total & $\pm 10.4 \%$
\end{tabular}

Table 5.18: Table of systematic uncertainties for the $B_{s}^{0} / B^{0}$ relative branching fraction measurements. 


\subsection{Measurement Result}

Section 5.5 deals with extracting the ratio of branching fractions from the measured $B_{s}^{0}$ and $B^{0}$ meson yields and relative efficiencies extracted from Monte Carlo. Section 5.6 reviews the systematic uncertainties of the measurement, and finds a total $10.4 \%$ relative systematic uncertainty on the measurement. We measure the ratio of the branching fraction of $B_{s}^{0} \rightarrow D_{s}^{-} \pi^{+}$decays relative to the branching fraction of $B^{0} \rightarrow$ $D^{-} \pi^{+}$decays multiplied by the ratio of production fractions for $B_{s}^{0}$ mesons relative to $B^{0}$ mesons $\left(f_{s} / f_{d}\right)$ to be:

$$
\frac{f_{s}}{f_{d}} \cdot \frac{B r\left(B_{s}^{0} \rightarrow D_{s}^{-} \pi^{+}\right)}{B r\left(B^{0} \rightarrow D^{-} \pi^{+}\right)}=0.325 \pm 0.046(\text { stat }) \pm 0.034(\text { syst }) \pm 0.084(B R)
$$

where the first uncertainty is due to statistics, the second is the systematic uncertainty on the measurement, and the third is due to the uncertainty of the world average [10] measurements of the $D_{s}^{-} \rightarrow \phi \pi$ and $D^{-} \rightarrow K^{+} \pi^{-} \pi^{-}$branching fractions. Using the world average [10] value of $f_{s} / f_{d}=0.26 \pm 0.03$, we infer that the ratio of branching fractions is:

$$
\frac{B r\left(B_{s}^{0} \rightarrow D_{s}^{-} \pi^{+}\right)}{B r\left(B^{0} \rightarrow D^{-} \pi^{+}\right)}=1.25 \pm 0.18(\text { stat }) \pm 0.13(\text { syst }) \pm 0.32(B R) \pm 0.14(P R)
$$

where the last uncertainty is due to the uncertainty on the world average measurement of the ratio of $B_{s}^{0}$ to $B^{0}$ production rates, $f_{s} / f_{d}$. 


\section{Chapter 6}

\section{$B_{s}^{0}$ Meson Yields}

The previous chapters dealt with understanding the relative rates of $B_{s}^{0} \rightarrow D_{s}^{-} \pi^{+}$ decays to $B^{0} \rightarrow D^{-} \pi^{+}$decays. In addition, it is also relevant to understand the absolute rate of reconstructed $B_{s}^{0} \rightarrow D_{s}^{-} \pi^{+}$decays, especially because the available sample size is significantly smaller than the one predicted by initial design studies [57]. Recently, dynamically prescaled trigger paths have been developed with the intention of increasing the $B$ meson yield by populating unused trigger bandwidth. From Monte Carlo simulation and results from data, we can infer the increase in $B$ meson yields due to these new trigger paths.

In the following sections, we briefly review the initial design study and its results, additional trigger and reconstruction effects which need to be taken into account to understand the current yield. In the last section we derive a new estimate of the number of $B_{s}^{0} \rightarrow D_{s}^{-} \pi^{+}$events which are expected per $\mathrm{fb}^{-1}$ of data, based on the currently reconstructed sample and known trigger and luminosity upgrades.

\subsection{Design Yield Studies}

In this section, we explain the procedure used to determine the initial projections for the yields of $B_{s}^{0} \rightarrow D_{s}^{-} \pi^{+}$decays. To simulate the SVT-based trigger system, a parametric simulation program is implemented, and nominal performance parameters for different trigger subsystems are used. This procedure is documented in detail 
elsewhere $[58,57]$. The benefit of using a parametric simulation is understanding the exact contribution of every effect simulated. Such flexibility is not available from the realistic simulation framework. Here, we briefly revisit the technical implementation of the trigger simulation and the estimated yields.

\subsubsection{Event Generation}

$B_{s}^{0}$ mesons are generated in a similar way to what was described in Section 5.1, with a few differences. The CLEO Monte Carlo Program QQ [59] is used to decay the $B_{s}^{0}$ mesons. The primary interaction region is simulated to be symmetric in $\varphi$ around the origin. The interaction intensity in this plane was modeled with a Gaussian distribution around the origin, with a width of $25 \mu \mathrm{m}$ in both the $x$ and $y$ directions. The $z$ distribution of the interaction region was modeled with a Gaussian distribution around the origin, whose width is $30 \mathrm{~cm}$.

\subsubsection{Level 1 Parametric Trigger Simulation}

Three elements of the Level 1 Trigger system are simulated: the XFT, the XTRP and the Level 1 trigger decision. The simulated $p_{T}$ resolution of the XFT is $\sigma\left(p_{T}\right) /\left(p_{T}^{2}\right)=$ $0.015 \mathrm{GeV}^{-1}$, and the simulated resolution of the $\varphi_{6}$ measurement is $1.5 \mathrm{mrad}$. Track parameters are smeared according to these input resolutions. The XFT is assumed to have a track finding efficiency of $97 \%$, regardless of track $p_{T}$ and $\varphi_{6}$. The XFT divides the COT into bins of $1.25^{\circ}$ in $\varphi_{6}$ and only the highest momentum track in a given bin is reported. The XFT output is further binned by the XTRP system. For a COT segment of $15^{\circ}$, only the two outermost tracks in $\varphi$ which have momenta greater than $2 \mathrm{GeV} / \mathrm{c}$ as measured in the XFT are reported to the Level 1 decision system. Both binning effects are fully simulated. The Level 1 selection criteria are already explained in Section 3.8, and we briefly repeat them here. The Level 1 system requires a track pair such that the tracks have opposite charge, each track satisfies $p_{T}>2.0 \mathrm{GeV} / \mathrm{c}$, and the sum $p_{T 1}+p_{T 2}>5.5 \mathrm{GeV} / \mathrm{c}$. The opening angle $\Delta \varphi_{6}<135$. The same selection criteria are imposed upon the simulated XFT track measurements. 


\subsubsection{Level 2 Parametric Trigger Simulation}

In order to simulate the SVT system, we define the fiducial volume of the SVX-II with a series of concentric cylinders. To simulate the mechanical support systems and the readout chips, which are passive detector parts, we define cracks in $z$ along these cylinders. If a track traverses a cylinder in the crack region, it is assumed not to leave a hit in the silicon system, and it is therefore lost to the SVT pattern recognition. The radii of the cylinders and the $z$ regions of the cracks are listed in Table 6.1. The SVT is assumed to have a hit finding efficiency of $98 \%$ per layer when a track traverses the active region of the layer, and a pattern recognition efficiency of $95 \%$ per track. These are design efficiencies for the SVT system. Requiring hits in the 4 layers of SVX in which we have SVT pattern recognition results in an overall efficiency of $\epsilon_{\text {tot }}=0.98^{4} \cdot 0.95=87.62 \%$ which is implemented as an overall efficiency. Tracks reconstructed using the SVT system have better resolutions than the XTRP tracks: $\sigma\left(p_{T}\right) / p_{T}^{2}=0.0013 \mathrm{GeV}^{-1}$ and $\sigma\left(\varphi_{0}\right)=1.5 \mathrm{mrad}$. The Level 1 event selection criteria are repeated using the new SVT tracks, and these have to be passed before the Level 2 conditions are applied. The Level 2 selection requirements are listed in Section 3.8. For this study we required that an event passes either the B_CHARM trigger selection or the $B \rightarrow \pi \pi$ trigger selection, so we list the criteria for the two triggers in Table 6.2 .

\subsubsection{Tracking Fiducial Volume Simulation}

For an event to be useful in the analysis, the $B$ meson has to be reconstructible in the detector. The requirement for a $B$ meson to be considered reconstructible in this simulation is that all its stable, charged daughter particles are within $|\eta|<1$ and have a transverse momentum greater than $400 \mathrm{MeV} / \mathrm{c}$.

\subsubsection{Yield Estimate}

Our yield estimate is based on a number of measured values which are used as input. All the input values and associated errors are listed in Table 6.3. Our result is 
normalized to the measured production cross section for $B^{0}$ mesons and $f_{s} /\left(f_{u}+f_{d}\right)$. We assume that the branching fraction for $B_{s}^{0} \rightarrow D_{s}^{-} \pi^{+}$is the same as the branching fraction for $B^{0} \rightarrow D^{-} \pi^{+}$. We use the world average values for the branching fractions of $D_{s}^{-} \rightarrow \phi \pi^{-}$and $\phi \rightarrow K^{+} K^{-}$. After requiring that the generated $B_{s}^{0}$ mesons pass the simulated trigger conditions and that all the daughter tracks of the $B_{s}^{0}$ meson be in the fiducial volume of the tracking detector, we expect to have 16,100 $B_{s}^{0} \rightarrow D_{s}^{-} \pi^{+}, D_{s}^{-} \rightarrow \phi \pi^{-}$decays reconstructed in $2 \mathrm{fb}^{-1}$ of data. A detailed list of efficiencies at every level of the simulation is listed in Table 6.4.

\subsection{Yield Reduction Effects}

When the SVT-based triggers were included in the data taking, a discrepancy was noticed with respect to the expected yields of $B$ mesons from the trigger. A detailed study of the rates of $B$ mesons coming from this trigger is difficult because the trigger underwent rapid development at the pattern-recognition level, which changes absolute efficiencies drastically and is hard to simulate well. Once the trigger development stabilized, it became possible to compare the performance of the SVT-based triggers to design expectations and determine differences. Several effects were found which differ from the design behavior of the trigger system and reduce rates. These are: regions of inactive silicon, wedge crossing effects, barrel crossing effects and trigger efficiencies being below design specifications. Each of these effects reduces the trigger efficiency slightly. Combined, they introduce a significant reduction of yields. In order to study these effects, we simulate them in our parametric Monte Carlo to estimate the individual impact, and then combine them to estimate the total yield reduction. A detailed description of the study is given elsewhere [60].

\subsubsection{Dead Silicon Regions}

The design performance of SVT-based triggers is calculated based on a silicon tracker which has no inactive chips. In reality, certain parts of the silicon system malfunctioned or got damaged by beam spills and had to be shut down. This causes a large 
problem to the SVT pattern recognition, because the pattern recognition requires a silicon hit to be present in each of the four innermost SVXII $r-\varphi$ layers. Shutting down a layer in a wedge of silicon renders the entire silicon wedge useless for SVT triggering. Figure 6-1 shows how the dead regions of the SVX reduce the number of SVT tracks reconstructed per wedge and halfladder. Introducing this effect into the simulation reduces the number of reconstructed $B_{s}^{0}$ candidates by about $20 \%$.

\subsubsection{Wedge Crossers}

The pattern recognition of the SVT system is based on the $\varphi$ symmetry of the SVXII system. Every wedge of the SVXII system covers $30^{\circ}$ of the $\varphi$ angle. Furthermore, if collisions happen in the center of the detector, particles with transverse momenta of $p_{T} \geq 2 \mathrm{GeV} / \mathrm{c}$ traverse all four layers of the SVXII system while staying in the same wedge. Only a small fraction of tracks crosses the boundary between wedges due to bending of the trajectory in the magnetic field and the finite size of the beamspot. The SVT pattern recognition takes advantage of this symmetry by only looking for tracks which have all four hits in the same wedge of the SVXII system. In reality, the beams are offset from the center of the detector by about $5 \mathrm{~mm}$. This results in an increased number of particles crossing wedge boundaries and not being reconstructed by SVT pattern recognition. Figure 6-2 shows the distribution of tracks not reconstructed because of wedge crossing effects. In the upper plot, the the beam is centered at the origin. In the lower plot, the physical beam displacement is correctly simulated. We find that this effect reduces our signal yields by about $25 \%$.

\subsubsection{Barrel Crossers}

In addition to crossing wedge boundaries, particles can also cross barrel and half-barrel boundaries and therefore not be reconstructed. The design SVT studies assume that tracks which cross both half-barrel or barrel boundaries are reconstructed. The SVT pattern recognition which is relevant to the data analyzed does not reconstruct either barrel or half-barrel crossers and a SVT track is lost as soon as the particle passes 
the first $z$ boundary. Figure 6-3 depicts this inefficiency. The upper plot shows the $\eta-z$ distribution of tracks lost due to tracks crossing an inactive region of the SVXII detector in one of the four layers, thus loosing one of the four required hits. The lower plot shows the same distribution, but this time tracks are also rejected if they cross half-barrel or barrel boundaries. One can see that the largest loss comes from not reconstructing tracks which cross half-barrel boundaries. We estimate that our signal loss due to this effect is about $15 \%$.

\subsubsection{Inefficiencies}

A certain set of hit and pattern finding inefficiencies are originally simulated based on design projections for the XFT and SVT systems. In particular, the XFT pattern recognition efficiency, the SVT hit finding efficiency and the SVT track reconstruction efficiencies are simulated using design predictions. We re-evaluate these efficiencies using unbiased data samples and find good agreement with the design predictions. However, in real data taking, a quality cut is placed on the SVT $\chi^{2}$ cut which was not simulated in the original yield projections. We find that the efficiency of the $\chi^{2}$ cut on track pairs in unbiased samples is $97 \%$. Table 6.5 lists a comparison of the design efficiencies and those which were found from tests on data. 


\begin{tabular}{l|c} 
Simulated Effect & Region in $|z|$ \\
\hline \hline half-barrel boundary & $0.0-1.125 \mathrm{~mm}$ \\
ladder boundary & $73.329-75.387 \mathrm{~mm}$ \\
mechanical support (bulkhead) & $147.591-166.204 \mathrm{~mm}$ \\
ladder boundary & $238.204-240.466 \mathrm{~mm}$ \\
half-barrel boundary & $312.670-314.920 \mathrm{~mm}$ \\
ladder boundary & $387.124-389.182 \mathrm{~mm}$ \\
end of fiducial volume & $461.386 \mathrm{~mm}$
\end{tabular}

Table 6.1: Gaps in the silicon coverage due to the mechanical support and readout chips. The end of the SVX-II fiducial volume is defined as $|z|=461.386 \mathrm{~mm}$.

\begin{tabular}{c|c} 
multi-body $B$ decays & $B \rightarrow \pi^{+} \pi^{-}$decays \\
\hline \hline $120 \mu \mathrm{m}<\left|d_{0}\right|<1 \mathrm{~mm}$ & $100 \mu \mathrm{m}<\left|d_{0}\right|<1 \mathrm{~mm}$ \\
$2^{\circ}<\Delta \varphi_{0}<90^{\circ}$ & $20^{\circ}<\Delta \varphi_{0}<135^{\circ}$ \\
$\overrightarrow{p_{T}} \cdot \vec{X}_{v}>0$ & $\overrightarrow{p_{T}} \cdot \vec{X}_{v}>0$ \\
- & $\left|d_{0}(B)\right|<140 \mu \mathrm{m}$
\end{tabular}

Table 6.2: Selection requirements applied for the two Level 2 trigger options

\begin{tabular}{l|c|l} 
Quantity & Value & Reference \\
\hline \hline$\sigma\left(B^{0}\right)\left(p_{T}\left(B^{0}\right)>6 \mathrm{GeV} / \mathrm{c} ;|\mathrm{y}|<1\right)$ & $(3.52 \pm 0.61) \mu \mathrm{b}$ & CDF Measurement [61] \\
$f_{s} /\left(f_{u}+f_{d}\right)$ & $0.213 \pm 0.038$ & CDF Measurement [62] \\
$B r\left(B_{s}^{0} \rightarrow D_{s}^{-} \pi\right)$ & $(3.0 \pm 0.4) \cdot 10^{-3}$ & from $B^{0}$ in PDG [10] \\
$B r\left(D_{s}^{-} \rightarrow \phi \pi^{-}\right)$ & $(3.6 \pm 0.9) \%$ & PDG [10] \\
$B r\left(\phi \rightarrow K^{+} K^{-}\right)$ & $(49.1 \pm 0.8) \%$ & PDG [10]
\end{tabular}

Table 6.3: Measured and estimated values used as input for the $B_{s}^{0} \rightarrow D_{s}^{-} \pi^{+}$yield estimate. 


\begin{tabular}{l|c} 
Effect & Value \\
\hline \hline Level 1 efficiency & $2.60 \%$ \\
Level 2 multi-body efficiency & $0.47 \%$ \\
Level 2 $B \rightarrow \pi^{+} \pi^{-}$efficiency & $0.29 \%$ \\
Combined Level 2 efficiency & $0.51 \%$ \\
Fiducial efficiency & $0.35 \%$ \\
\hline Event yield & 16,100
\end{tabular}

Table 6.4: Efficiencies and yield estimate for $B_{s}^{0} \rightarrow D_{s}^{-} \pi^{+}$decays in $2 \mathrm{fb}^{-1}$ of data.

\begin{tabular}{l|c|c} 
Efficiency & Design Estimate & Measured \\
\hline \hline XFT pattern recognition & $97 \%$ & $97 \%$ \\
SVT hit finding & $95 \%$ & $94 \%$ \\
SVT pattern recognition & $95 \%$ & $94 \%$ \\
SVT $\chi^{2}$ requirement & - & $97 \%$ \\
\hline Total & $85 \%$ & $82 \%$
\end{tabular}

Table 6.5: Comparison of design trigger-level efficiencies to those measured in unbiased data samples [60].

\begin{tabular}{l|c|r|r} 
Effect & Efficiency & Efficiency Loss & Cumulative Loss \\
\hline \hline Initial Study & $2.87 \pm 0.04 \%$ & - & - \\
SVT $\chi^{2}$ requirement & $2.52 \pm 0.04 \%$ & $12 \%$ & $12 \%$ \\
Beam displacement & $1.79 \pm 0.04 \%$ & $26 \%$ & $37 \%$ \\
Barrel boundary crossers & $1.77 \pm 0.04 \%$ & $1 \%$ & $38 \%$ \\
Half-barrel crossers & $1.54 \pm 0.04 \%$ & $8 \%$ & $46 \%$ \\
Dead regions of SVXII & $1.30 \pm 0.04 \%$ & $9 \%$ & $55 \%$
\end{tabular}

Table 6.6: Reduction of the trigger efficiency as new effects are included in the parametric simulation [60]. 


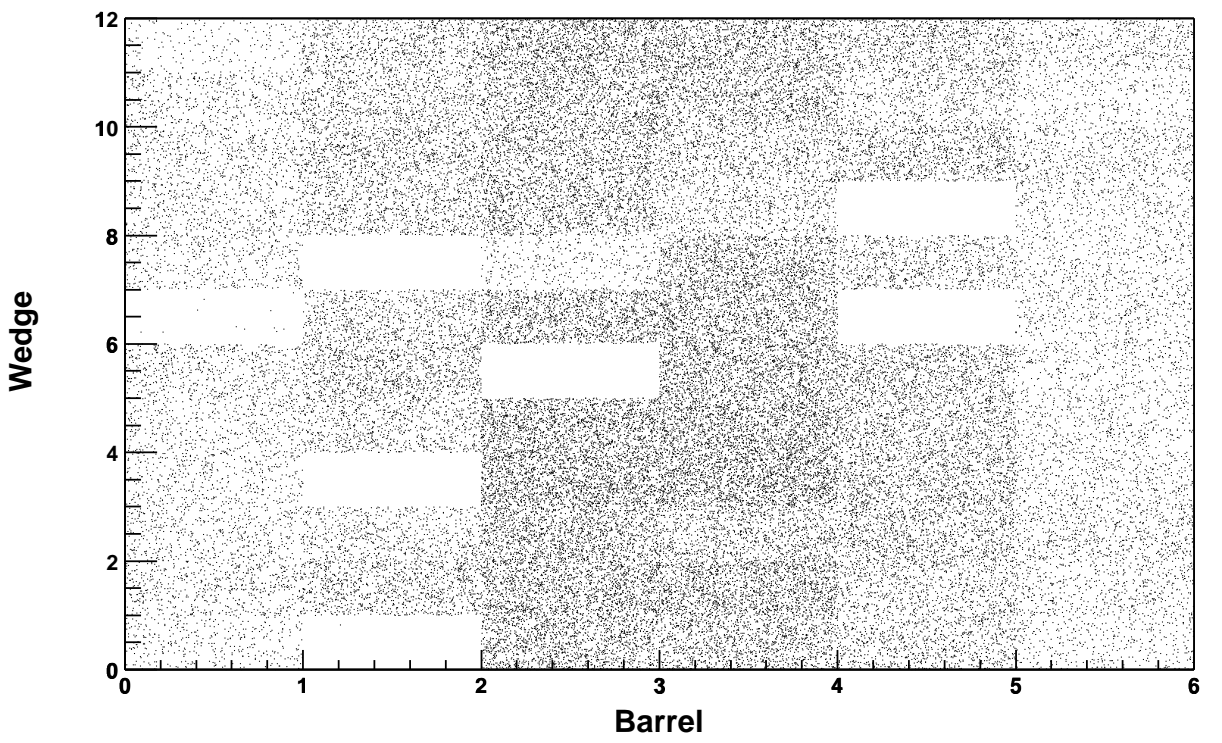

Figure 6-1: Track hit occupancy in wedges and halfbarrels of the SVX. For every point in the plot, a track with SVX hits has been matched to an SVT track. Areas with no points have no tracks because one of the layers of Silicon are not functioning. 

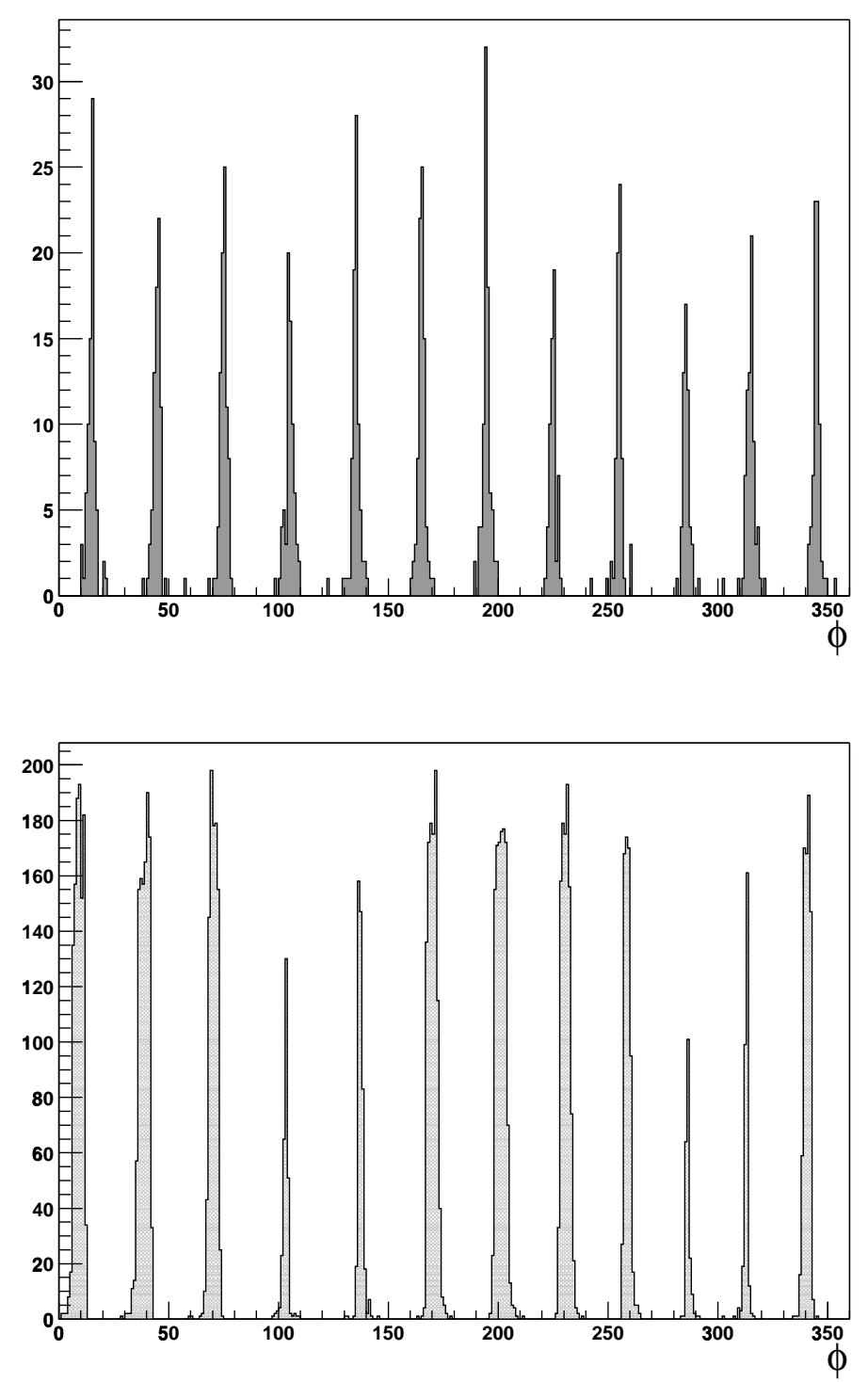

Figure 6-2: Tracks lost due to wedge boundary crossing. The top plot shows the distribution of the $\varphi_{0}$ of the tracks lost due to wedge boundary crossing when the interaction point is at the center of the detector. The bottom plot shows the same distribution when the interaction point is displaced by $5 \mathrm{~mm}$ from the center of the detector. Notice the difference in $y$ scales of the two plots. 

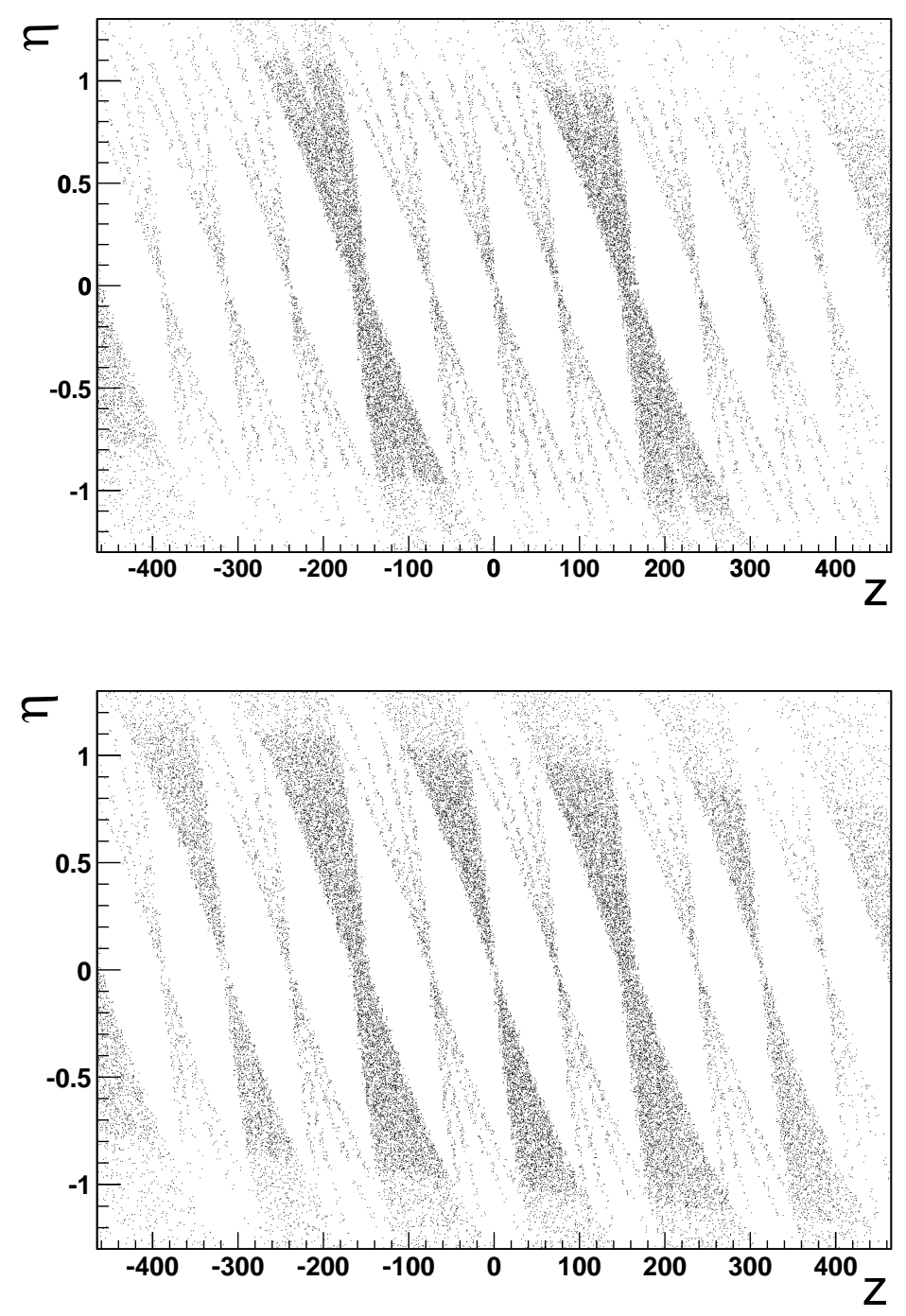

Figure 6-3: Tracks lost due to barrel and half-barrel boundary crossing. The top plot shows the distribution of tracks which are lost because they traversed an inactive region of the SVX-II system in one of the four layers. This is the design behavior of the SVT. The bottom plot shows the same distribution for the pattern recognition relevant to our sample; tracks which cross half-barrel or barrel boundaries are not reconstructed. Signal loss due to this effect is about $15 \%$. 


\subsubsection{Yield Reduction}

In order to estimate the total trigger yield reduction, we implement all the new trigger effects in our parametric simulation and check the yield reduction as each individual effect gets included. Table 6.6 lists the reduction of the trigger efficiency as different effects are included in the trigger simulation. Some of the effects are correlated so the overall yield reduction is not just a direct product of different effect efficiencies. We find that the trigger efficiency is reduced by $55 \%$ when all the new effects are introduced.

It should also be noted that this comparison of trigger efficiencies is done for a period in which the trigger conditions were stable. When the trigger was first introduced into the data taking, the hit and pattern recognition algorithms were much less efficient. On the other hand, due to rapid development of the algorithms during this commissioning phase, the efficiency also improved very quickly. We estimate that the trigger efficiency doubled due to this initial trigger development. To correct for these trigger development effects, we introduce another inefficiency of 0.5 to our yield estimate.

In addition to the decreased trigger efficiency, our initial estimate did not take into account any analysis inefficiencies. These inefficiencies are introduced by selection requirements and are necessary to extract a clean signal. We compare the fiducial efficiency of the original yield study, listed in Table 6.4 to the estimated reconstruction efficiency for our signal, listed in Table 5.11, and find that the effect of using selection requirements introduces another inefficiency of $47 \%$ to our yields.

As described earlier, prior to this measurement there was no good estimate of the value of $f_{s} / f_{d} \cdot \operatorname{Br}\left(B_{s}^{0} \rightarrow D_{s}^{-} \pi^{+}\right) / \operatorname{Br}\left(B^{0} \rightarrow D^{-} \pi^{+}\right)$. Our initial yield estimate used the measured $B^{+}$production cross section, the measured value of $f_{s} / f_{d}=0.43$ and $\operatorname{Br}\left(B_{s}^{0} \rightarrow D_{s}^{-} \pi^{+}\right)=\operatorname{Br}\left(B^{0} \rightarrow D^{-} \pi^{+}\right)$to predict the $B_{s}^{0}$ yield. Effectively, this meant that we assumed:

$$
\frac{f_{s}}{f_{d}} \cdot \frac{B_{s}^{0} \rightarrow D_{s}^{-} \pi^{+}}{B^{0} \rightarrow D^{-} \pi^{+}}=0.43
$$


The measurement described in the previous chapters shows that we have measured a value of 0.33 instead. This means that we should correct the initial estimate by a factor $0.33 / 0.43=0.77$ to compensate for this effect.

Finally, the initial estimates predicted rates based on the assumption that both the B_CHARM trigger path and the trigger path for reconstructing $B \rightarrow \pi^{+} \pi^{-}$decays are used to reconstruct $B_{s}^{0}$ decays. Our study only used the B_CHARM trigger path, which accounts for $90 \%$ of the expected $B_{s}^{0}$ yield. All the correction factors and corrected yields are listed in Table 6.7. After applying all effects, we find that the expected number of reconstructed $B_{s}^{0}$ candidates in our data sample should be 90 , and we reconstruct 80 candidates which is in good agreement with the prediction.

\begin{tabular}{l|cr} 
Effect & Corr. Factor & $B_{s}^{0}$ Yield \\
\hline \hline Initial Estimate $\left(2 \mathrm{fb}^{-1}\right)$ & - & 16100 \\
Integrated $\mathcal{L}\left(115 \mathrm{pb}^{-1}\right)$ & $0.115 / 2$ & 930 \\
Trigger development & $\simeq 0.5$ & 460 \\
Unaccounted Trigger Effects & 0.54 & 250 \\
Selection Requirements & 0.53 & 130 \\
$f_{s} / f_{d}$ and Branching Ratios & $0.33 / 0.43$ & 100 \\
Not using the $B \rightarrow \pi \pi$ trigger & 0.9 & 90
\end{tabular}

Table 6.7: Breakdown of event loss due to different effects which were not taken into account in the initial yield estimate. 


\subsection{Triggers With Loose Requirements}

In order to increase the $B$ meson yield, an additional trigger path has been developed. The path name for this trigger is B_CHARM_LOWPT and it is dynamically prescaled to take advantage of available trigger bandwidth as described in Section 4.2.2. The B_CHARM and LOW_PT triggers are running with dynamic prescaling for most of our data taking. B_CHARM typically rejects about $5 \%$ of the events due to dynamic prescaling. The LOW_PT trigger rejects $50 \%$ events in the hbot0h sample, and about $25 \%$ of events in the hbot1i sample because of prescaling. Because of the different prescaling rates of the two triggers, introducing events from the LOW_PT path into our measurements could compromise the validity of rate measurements. However, since we would like to understand our total $B$ meson yields, we try to estimate the yield increase due to these additional triggers.

Figure 6-4 illustrates the overlaps of the different triggers that we study. The phase space of the B_CHARM trigger is a subspace of the LOW_PT trigger, and the phase space of the HIGH_PT trigger is a subspace of both. The effective prescale depicted on the $y$ axis reflects that the same fraction of events are lost for a given trigger across its phase space. The LOW_PT trigger loses most events due to prescaling, followed by the B_CHARM trigger. The HIGH_PT trigger path is not prescaled in current data taking, but it will be prescaled in the future with increased Tevatron luminosities $\left(8 \cdot 10^{31} \mathrm{~cm}^{-2} \mathrm{~s}^{-1}\right.$ and higher $)$.

\subsection{Updated Yield Projections}

Several changes have recently been made to the trigger that improved the yields. In this study we assume that the following changes will work (they have already been tested to a large extent):

- Implementation of the 4 out of 5 scheme for the SVT trigger.

- Implementation of full SVX tracking in the level 3 filter and confirmation of the SVT trigger using SVX tracks. 
- Implementation of the data compression scheme on the level $3 \mathrm{PC}$ farm which lowers the limitation on the rate of data writing to tape

To extrapolate, we are using the numbers obtained in the Section 5.2.6 as a starting point and use partially data and partially realistic Monte Carlo simulation to take into account the various improvements.

The number of $B$ mesons used in the branching ratio analysis is not necessarily the same number we expect to obtain for the $B_{s}^{0}$ mixing analysis. The confirmation of the trigger becomes much less of an issue. Matching tracks to the SVT is irrelevant since non $B_{s}^{0}$ tracks might have triggered the event. To stay on the conservative side we will nevertheless not change the analysis cuts since it is difficult to extrapolate numbers when the origin of the trigger is not well defined. This makes the estimate slightly conservative.

The following adjustments are made to the reconstructed number of $B_{s}^{0}$ events to properly predict the cross section for reconstructed $B_{s}^{0}$ events. The number of events has to be divided by the luminosity, $\mathcal{L}$ to obtain the cross section. The hbotoh detector inefficiencies and the SVT tuning for the hbot1i have to be accounted for, $R_{\text {pre }}$ and the expected improvements from the 4 out of 5 algorithm, $R_{4 / 5}$. For the additional events from the dynamically prescaled trigger, we estimate the ratio of additional events without prescale, $A_{\mathrm{dps}}$, and divide by the effective prescale factor, $F_{\text {dps }}$.

$$
\sigma\left(B_{s}^{0}\right)=\left(N\left(B_{s}^{0}\right) / \mathcal{L}\right) \times R_{\mathrm{pre}} \times R_{4 / 5} \times\left(1+A_{\mathrm{dps}} / F_{\mathrm{dps}}\right)
$$

The prescale factor, $F_{\text {dps }}$, has to be integrated with luminosity weighting and according to the instantaneous luminosities. We consider two scenarios to evaluate the uncertainties on the prescaled trigger paths. The scenarios are as follows:

- realistic: Level 1 rate sustainable up to $20 \mathrm{kHz}$, the current luminosity profile which peaks around $2 \times 10^{31} 1 /\left(\mathrm{cm}^{2} \mathrm{~s}\right)$ and has a maximum at about $4 \times$ $10^{31} 1 /\left(\mathrm{cm}^{2} \mathrm{~s}\right)$

- optimistic: level 1 rate sustainable up to $25 \mathrm{kHz}$ and the current luminosity 
profile but stretched by a factor of 1.5: peaks around $3 \times 10^{31} /\left(\mathrm{cm}^{2} \mathrm{~s}\right)$ and has a maximum at about $6 \times 10^{31} /\left(\mathrm{cm}^{2} \mathrm{~s}\right)$

\subsubsection{Preshutdown Inefficiencies and SVT Improvements}

In the beginning of data taking the silicon detector and SVT were running in a suboptimal way. Parts of the silicon detector were not powered or did not take data properly. Furthermore, the treatment of dead ladders in the silicon has been significantly improved after the shutdown. After the correction, we obtain the reconstructed $B_{s}^{0}$ cross section corresponding to data taking with the detector in postshutdown conditions. Therefore, we calculate the efficiency correction weighted with the luminosities which is applied as a multiplicative factor, $R_{\text {pre }}$ :

$$
R_{\mathrm{pre}}=\frac{\sum \mathcal{L}_{i} \epsilon_{\mathrm{post}} / \epsilon_{i}}{\sum \mathcal{L}_{i}}
$$

The trigger efficiencies, $\epsilon_{i}$, for the different periods, are quoted in Table 5.9 and the luminosities per period are listed in Table 5.3. The trigger efficiency we want to normalize to is the average trigger efficiency in the hbot1i dataset $\epsilon_{\text {post }}$, which is listed in Table 5.11.

Using the luminosities and the trigger efficiencies from the realistic Monte Carlo for $B_{s}^{0} \rightarrow D_{s}^{-} \pi^{+}$the appropriate scale factor comes out to be: $R_{\text {pre }}=1.2$. When studying the improvement in $B$ event yields in our data a factor of about 1.3 is obtained. We use the factor of 1.3 which was measured in data and use the difference between data and Monte Carlo prediction as a systematic uncertainty, \pm 0.1 .

\subsubsection{SVT Algorithm Four out of Five}

The SVT four out of five algorithm loosens the original SVT requirement that there are hits found in the innermost four SVT layers. Instead, hits should be found in four of the five SVX layers of a given wedge for SVT pattern recognition to reconstruct a track. The algorithm has been tested successfully on data. From yield measurement using $J / \psi$ and $D^{0}$ data an increase of a factor of 1.5 has been observed. We have also 
extracted numbers from the realistic Monte Carlo simulation. The trigger efficiency increases by a factor of about 1.6 and the yield correction stays constant after applying full reconstruction cuts. Conservatively, we use a factor of $R_{4 / 5}=1.5$ since it has been measured in data with high statistics. We use the difference between data and Monte Carlo prediction as a systematic uncertainty, \pm 0.1 .

\subsubsection{Dynamically Prescaled Triggers}

The B_CHARM_LOWPT trigger path is used to recover lower $p_{T} B$ decays in times when the instantaneous luminosity is low and the bandwidth is not filled with the standard triggers. The prescale factors are reduced as soon as bandwidth becomes available.

The realistic Monte Carlo simulation predicts that the dynamically prescaled B_CHARM_LOWPT trigger path increases the yields by additional factor of $A_{\text {pre }}=0.66$. This does not imply any prescale factor yet. In the postshutdown data with the given luminosity profile the prescale factor turns out to be about $R_{\mathrm{dps}}=1.5$.

As the prescale is dynamic and depends on the instantaneous luminosity the prescale factor depends on the actual distributions of instantaneous luminosities throughout the data taking period. The luminosity profile for data taking is the distribution of luminosity per run section (smallest, indivisible segment of a dataset) for a given time period. A profile for the instantaneous luminosity is shown in Figure 6-5. This profile is provided for the month of May 2003, which was the best data taking month for CDF-II at the time of this study.

With the present characteristics of CDF-II's data acquisition the prescale further depends on the maximal Level 1 trigger input rate. The prescale factor, $F_{d p s}$, is

$$
F_{d p s}=1 / \max \left(0, \min \left(1,\left(r_{L 1, \max }-329 \cdot L\right) /(400 \cdot L)\right)\right)
$$

and is shown as a function of the instantaneous luminosity, $L$, for various maximal Level 1 trigger rates, $r_{L 1, \max }$, in Figure 6-7. A convolution of the prescale factor at a given instantaneous luminosity with the luminosity profile reveals the effective prescale factor which we are interested in. 
Using a maximum Level 1 trigger rate of $15 \mathrm{kHz}$ the convolution with the May luminosity profile results in a prescale factor of 1.7. This factor is somewhat higher than the effective prescale factor for the complete postshutdown period because we selected the best month. The prescale factor for the overall postshutdown period comes out to be about about 1.5 [63].

For our realistic extrapolation scenario we assume the month of May luminosity profile and the achieved Level 1 trigger rate limit of $20 \mathrm{kHz}$. The convolution results in a factor of 1.2 .

To evaluate a scenario with a high Tevatron instantaneous luminosity we take the luminosity profile of May and stretch all instantaneous luminosities by a factor of 1.5. The distribution then reaches up to a maximum instantaneous luminosity of about $60 \times 10^{30} \mathrm{~cm}^{-2} \mathrm{~s}^{-1}$ ) which is close to the design goal. By the time Tevatron will have achieved this luminosity the Level 1 rate limit will have gone up to about $25 \mathrm{kHz}$. The convolution results in a prescale factor of 1.5.

It is difficult to quote a systematic error on the effective prescale since the Tevatron performance (luminosity profile) as well as the Level 1 trigger rate limit are not well known. The uncertainty is reflected in the two different scenarios quoted. 


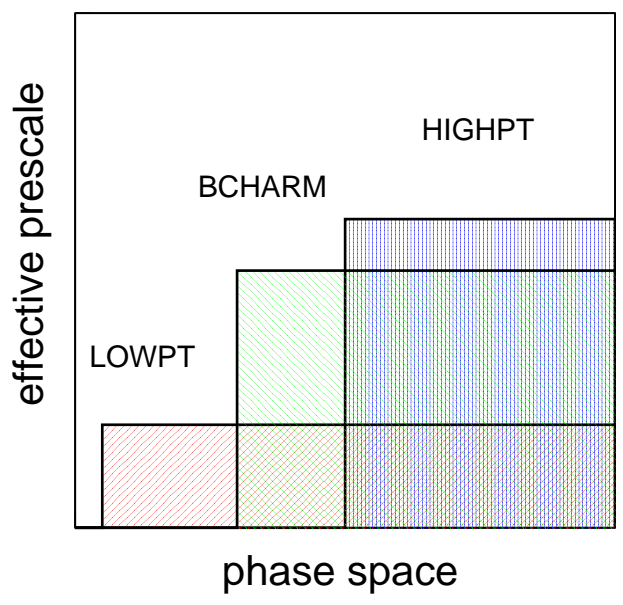

Figure 6-4: A sketch of the phase space overlaps of the three main hadronic $B$ paths.

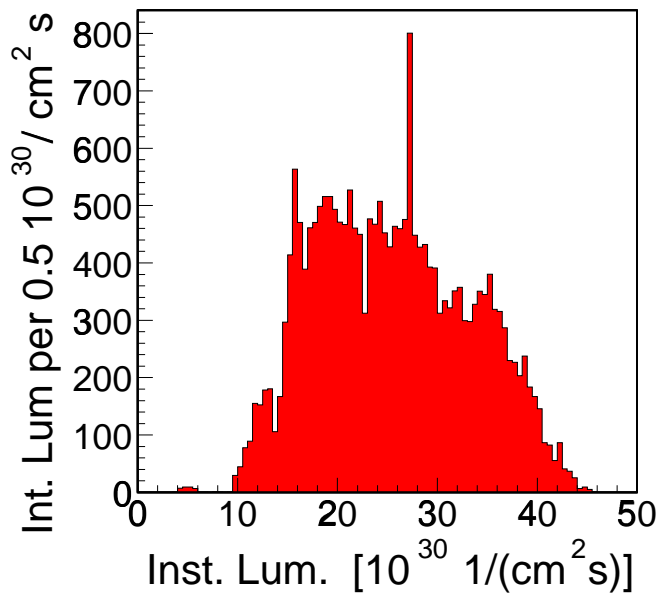

Figure 6-5: Profile of instantaneous luminosities in the Month of May 2003, which was the best month of data taking so far. The picture shows how much luminosity has been integrated at a given instantaneous luminosity. 


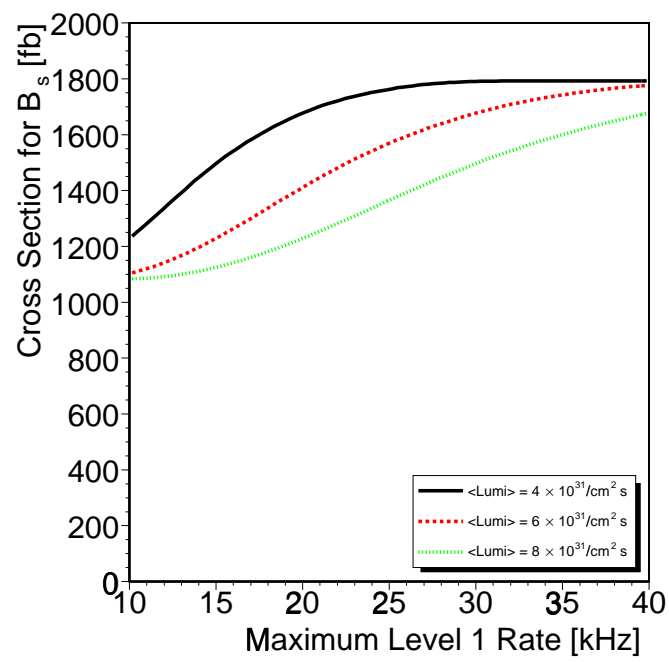

Figure 6-6: The effective $B_{s}$ cross section as a function of the Level 1 rate limitation. Three scenarios with different average instantaneous luminosity profiles are shown.

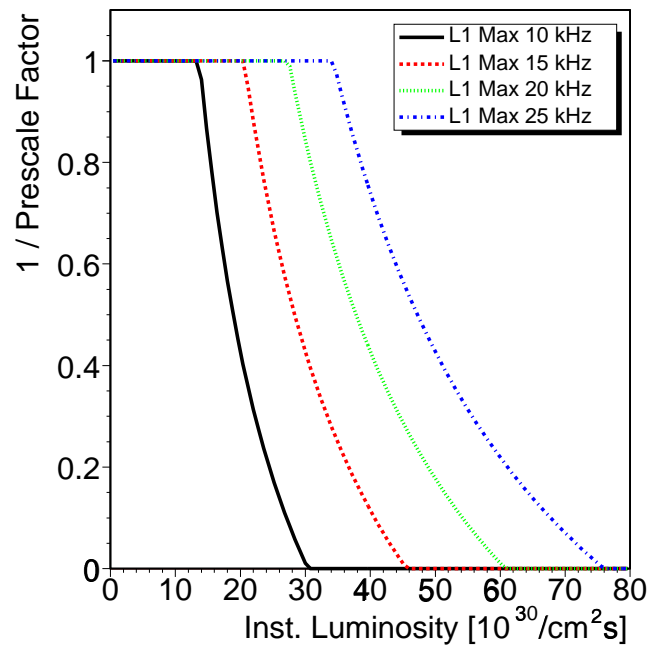

Figure 6-7: The inverse of the prescale factor in dependence of the instantaneous luminosity. Four different maximal Level 1 trigger rates are depicted. The prescale factor is 1 as long as the Level 1 trigger rate is not saturated, and gets increased to only fill the remaining trigger bandwidth. 


\subsubsection{Level 1 Rate Limitation}

The prescale factor for the dynamically prescaled triggers depends both on the instantaneous luminosity as well as on the rate limitation of the Level 1 trigger. Depending on the profile of the instantaneous luminosity the rate limitation can have a quite substantial effect on the $B_{s}$ event yields. To demonstrate this behavior we assume three luminosity profiles with peaks at $40 \times 10^{30} \mathrm{~cm}^{-2} \mathrm{~s}^{-1}, 60 \times 10^{30} \mathrm{~cm}^{-2} \mathrm{~s}^{-1}$ and $80 \times 10^{30} \mathrm{~cm}^{-2} \mathrm{~s}^{-1}$. The effective $B_{s}$ cross sections versus the Level 1 rate limitation is shown in Figure 6-6. For very low Level 1 rate limitations the dynamic prescaled trigger contributions basically vanish while they are almost not prescaled when the Level 1 rate limitations are large.

The maximal effect is thus the addition of $66 \%$ as discussed in the last subsection. For the profile with instantaneous peak luminosities of $40 \times 10^{30} \mathrm{~cm}^{-2} \mathrm{~s}^{-1}$ the increase in effective cross section between $20 \mathrm{kHz}$ and $40 \mathrm{kHz}$ is about $6 \%$ while for the profile with instantaneous peak luminosities of $60 \times 10^{30} \mathrm{~cm}^{-2} \mathrm{~s}^{-1}$ the increase is $27 \%$.

\subsubsection{Projected Yields}

The best estimate of the number of events in the B_CHARM trigger path for the preshutdown data is provided in Section 5.2.6: $42 \pm 7$. This number is going to be used in our formula. The two scenarios are derived from the numbers quoted in the previous subsections applying the formula in Equation 6.2. The first so called realistic scenario has

$$
\sigma\left(B_{s}^{0}\right)=36 \times 1 / 0.065 \mathrm{fb}^{-1} \times 1.30 \times 1.50(1+0.66 / 1.2)=1953 \mathrm{fb}
$$

For the second scenario, the optimistic, only the effective prescale factor has to be changed to 1.5:

$$
\sigma\left(B_{s}^{0}\right)=36 \times 1 / 0.065 \mathrm{fb}^{-1} \times 1.30 \times 1.50(1+0.66 / 1.5)=1814 \mathrm{fb}
$$

For the optimistic scenario the reconstructed $B_{s}^{0}$ cross section turns out to be lower but the luminosity is integrated in half the time, which justifies the word optimistic. 
For the extrapolation we suggest to use the average of those two scenarios which is $1900 \mathrm{fb}$. The uncertainties on those numbers are not small. The uncertainties on the number of reconstructed $B_{s}^{0}$ events, the correction of the preshutdown detector conditions, the $4 / 5$ extrapolation and the dynamic prescales added in quadrature reveals a total uncertainty of $(19 \% \oplus 10 \% \oplus 10 \% \oplus 5 \%)=24 \%$ corresponding to about $400 \mathrm{fb}$. This cross section only includes the decay $B_{s}^{0} \rightarrow D_{s}^{-} \pi^{+}$with $D_{s}^{-} \rightarrow \phi \pi^{-}$and $\phi \rightarrow K^{+} K^{-}$.

\subsection{Conclusions}

We have reviewed the design projections for the $B_{s}^{0} \rightarrow D_{s}^{-} \pi^{+}$yield and listed a number of effects that could not be quantified at the time. After correcting for these effects, we find reasonable agreement between the number of $B_{s}^{0}$ mesons reconstructed in data and predicted by Monte Carlo. From the yields obtained in data, the expected luminosity profile and the known and expected trigger upgrades, we expect to accumulate $1900 B_{s}^{0} \rightarrow D_{s}^{-} \pi^{+}$decays per $\mathrm{fb}^{-1}$ of data gathered. 


\section{Chapter 7}

\section{Conclusion}

We analyze $115 \mathrm{pb}^{-1}$ of data collected with the upgraded CDF-II detector. By triggering on displaced tracks, we gather a sample rich in bottom and charm mesons. In this sample, we make the first high statistics observation of the hadronic decay $B_{s}^{0} \rightarrow D_{s}^{-} \pi^{+}, D_{s}^{-} \rightarrow \phi \pi^{-}$. These decays provide excellent measurements of the $c t$ of

the $B_{s}^{0}$ meson decay and represent groundbreaking work for a future measurement of the frequency of $B_{s}^{0}$ oscillations.

Using a series of selection requirements optimized on a combination of data and Monte Carlo, we reconstruct $78 \pm 11 B_{s}^{0} \rightarrow D_{s}^{-} \pi^{+}$decays and $1153 \pm 45 B^{0} \rightarrow D^{-} \pi^{+}$ decays with good signal to background ratio. This is the world's largest sample of fully reconstructed $B_{s}^{0} \rightarrow D_{s}^{-} \pi^{+}$decays. After correcting for differences in trigger and reconstruction efficiencies, we find that the ratio of production fractions multiplied by the ratio of branching fractions for these decays is:

$$
\frac{f_{s}}{f_{d}} \cdot \frac{B r\left(B_{s}^{0} \rightarrow D_{s}^{-} \pi^{+}\right)}{B r\left(B^{0} \rightarrow D^{-} \pi^{+}\right)}=0.325 \pm 0.046(\text { stat }) \pm 0.034(\text { syst }) \pm 0.084(B R)
$$

where the first uncertainty is statistical, the second due to systematic effects, and the third is due to the current uncertainty on the world average $D$ branching fractions.

Using the world average [10] value of $f_{s} / f_{d}=0.26 \pm 0.03$, we infer that the ratio of branching fractions is: 


$$
\frac{B r\left(B_{s}^{0} \rightarrow D_{s}^{-} \pi^{+}\right)}{B r\left(B^{0} \rightarrow D^{-} \pi^{+}\right)}=1.25 \pm 0.18(\text { stat }) \pm 0.13(\text { syst }) \pm 0.32(B R) \pm 0.14(P R)
$$

where the last uncertainty is due to the uncertainty on the world average measurement of the ratio of $B_{s}^{0}$ to $B^{0}$ production rates, $f_{s} / f_{d}$.

This is in agreement with the theoretical prediction of equal branching fractions, and the measurement is currently limited by the world average measurement of the branching fraction $\operatorname{Br}\left(D_{s}^{-} \rightarrow \phi \pi^{-}\right)$. A better measurement of this branching fraction immediately improves our measurement, as it propagates directly into the rate calculation. We expect the systematic uncertainty on the measurement to improve as more data become available. Higher statistics are needed to test the agreement between the theoretical prediction and the experimental value.

The rate of observed $B_{s}^{0}$ mesons is significantly lower than predicted by design studies. By evaluating our current known trigger and reconstruction effects, we find that the discrepancy is consistent with a combination of effects which could not be taken into account in the design studies. We find that a fraction of these effects can be recovered by upgrading trigger algorithms, expanding trigger paths and correctly positioning the beam in the center of the detector. 


\section{Bibliography}

[1] N. Cabibbo, Phys. Rev. Lett. 10, 531 (1963).

[2] S.L. Glashow, J. Iliopoulos, and L. Maiani, Phys. Rev. D2, 1585 (1970).

[3] J.J Aubert et al., Experimental Observation of a Heavy Particle $J$, Phys. Rev. Lett. 33:1404-1406, (1974).

[4] J.E. Augustin et al., Discovery of a Narrow Resonance in $e^{+} e^{-}$Annihilation, Phys.Rev.Lett.33:1406-1408, (1974).

[5] M. Kobayashi and T. Maskawa, Progr. Theor. Phys. 49, 652 (1973).

[6] J.H. Christenson et al., Phys. Rev. Lett. 13, 138 (1964).

[7] S.W. Herb et al., Phys. Rev. Lett. 39, 252 (1977).

[8] F.Abe et al., Phys. Rev. Lett. 74, 2626 (1995).

[9] L. Wolfenstein, Phys. Rev. Lett. 51, 1915 (1983).

[10] Particle Data Group, K. Hagiwara et al., Phys. Rev. D 66, 010001 (2002).

[11] L.L. Chau and W.Y. Keung, Phys. Rev. Lett. 53, 1802 (1984).

[12] M. Battaglia et al., hep-ph/0304132.

[13] D. Boutigny et al., SLAC-R-0457.

[14] M.T. Cheng et al., BELLE-TDR-3-95, KEK-95-1. 
[15] S.Rahatlou et al, Measurement of the CP Violating Asymmetry Amplitude sin $2 \beta$ with the BaBar Detector., hep-ex/0207088.

[16] A. Abashian et al. Measurement of the CP Violation Parameter $\sin 2 \phi_{1}$ in $B_{d}^{0}$ Meson Decays, Phys.Rev.Lett. 86:2509-2514, (2001).

[17] BaBar Collaboration (P.F. Harrison, (ed.) et al.), SLAC-R-0504, 43-71 (1998).

[18] M. Ciuchini et al., Unitarity Triangle Analysis in the Standard Model and Sensitivity to new Physics, hep-ph/0307195.

[19] H.G. Moser, A. Roussarie, Nucl.Instrum.Meth. A384 491-505 (1997).

[20] PDG Review: Pseudoscalar meson decay constants, K. Hagiwara et al., Phys. Rev. D66, 010001 (2002).

[21] BaBar Collaboration (P.F. Harrison, (ed.) et al.), SLAC-R-0504, 63-64 (1998).

[22] S. Godfrey and N. Isgur, Phys. Rev. D 32, 189 (1985).

[23] G. Aubrecht et al., A Teachers Guide to the Nuclear Science Wall Chart, Contemporary Physics Education Project, 2003. http://www.lbl.gov/abc/wallchart/teachersguide/pdf/Chap11.pdf.

[24] C.W. Schmidt, The Fermilab 400-MeV Linac upgrade FERMILAB-CONF-93-111 (1993).

[25] Fermilab Beams Division, Run II Handbook, http://www-bd.fnal.gov/runII/index.html.

[26] J. Marriner, Stochastic Cooling Overview, FERMILAB-CONF-03-158(2003).

[27] R. Blair et al., The CDF-II detector: Technical design report, FERMILAB-PUB-96/390-E (1996).

[28] F. Abe et al., Nucl. Inst. and Meth. Phys. Res, 271A, 387 (1988); FERMILAB-PUB-94/024-E (1994). 
[29] T.K. Nelson et al.,

FERMILAB-CONF-01/357-E.

[30] A. Sill et al., Nucl. Instrum. Meth., A447, 1-8 (2000).

[31] T. Affolder et al., Nucl. Instrum. Meth., A485 , 6-9 (2002).

[32] K.T. Pitts et al.,

FERMILAB-CONF-96-443-E.

[33] R. Wagner et al., CDF Central Outer Tracker, CDF Note 6267 (2003).

[34] R. Brun, K. Hakelberg, M. Hansroul, and J.C. Lasalle, CERN-DD-78-2-REV; CERN-DD-78-2.

[35] G. Gomez for the TOF group, The CDF-II Time of Flight Detector, CDF Note 6258 (2003).

[36] P. Schlabach, CDF Central Muon Detector, http://www-cdf .fnal.gov/internal/upgrades/muon/cmu.html.

[37] P. Schlabach, CDF Central Muon Detector, http://www-cdf.fnal.gov/internal/upgrades/muon/cmp.html.

[38] P. Schlabach, CDF Central Muon Detector, http://www-cdf.fnal.gov/internal/upgrades/muon/cmx.html.

[39] E.J. Thomson et al., IEEE Trans. Nucl. Sci 49, 1063 (2002).

[40] W. Ashmanskas et al., Nucl. Instrum Methods Phys Res. A 447, 218 (2000), W. Ashmanskas et al., FERMILAB-CONF-02/035-E, A. Bardi et al., Nucl. Instrum Methods Phys Res. A 485, 6 (2002).

[41] I. Yu, J. Lewis, Study of $B \rightarrow \pi^{+} \pi^{-}$trigger rate for Run II, CDF Note 4095.

[42] A. Belloni, I.K. Furic, Ch. Paus, Multibody Trigger Paths in the Two Track Trigger Data, CDF Note 6526. 
[43] W. Badgett, The CDF Run II Run Database and Online Java API, CDF Note 5672.

[44] M. Feindt, S. Menzemer, K. Rinnert, P. Schemitz, A. Skiba, KalKalmanFitter A Fast and Versatile Kalman Track-Fitter for CDF II CDF Note 5388.

[45] B. Ashmanskas, A. Cerri, see CdfCode browser: http://cdfcodebrowser.fnal.gov/CdfCode/source/svtsim/ .

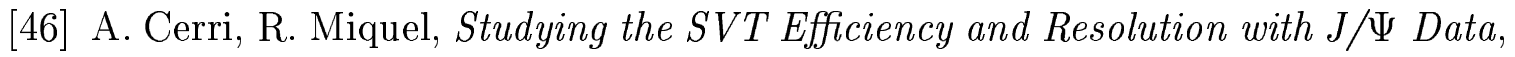
CDF Note 5838.

[47] J. Marriner, Secondary Vertex Fit with Mass and Pointing Constraints (CTVMFT), CDF Note 1996 (1993).

[48] K. Anikeev, P. Murat, Ch. Paus, Description of Bgenerator II, CDF Note 5092.

[49] P. Nason, S. Dawson and R.K. Ellis, Nucl. Phys. B303 (1988) 607; Nucl. Phys. B327 (1989) 49.

[50] C. Peterson et al., Phys. Rev. D27 (1983) 105.

[51] M. Cacciari et al., QCD Analysis of First B Cross-Section Data at $1.96 \mathrm{TeV}$, hep-ph/0312132.

[52] W. Bell, J.P. Fernandez, L. Flores, F. Wuerthwein, R.J. Tesarek, User Guide For EvtGen CDF, CDF Note 5618.

[53] A. Cerri, M. Rescigno, The CDFII Realistic Simulation Package - CDF Note in preparation.

[54] S. Giagu et al., BR ratios and direct CP violation in Cabibbo supressed decays of $D^{0}$, CDF Note 6391.

[55] K. Anikeev et al., An Update of B Lifetime Measurements Using Exclusively reconstructed Decays $B \rightarrow J / \psi X$ in Run II Data, CDF Note 6550. 
[56] M. Jones, Initial Performance Studies of the CDF-II Time-of-Flight Detector, CDF Note 5930.

[57] I.K. Furić, Ch. Paus, Event Yield Estimates for Hadronic Bs Decays in Run II, CDF Note 5349.

[58] I.K. Furić, Ch. Paus, C.J. Seely, Track Trigger Simulation at the Generator Level in Run II, CDF Note 5348.

[59] P. Avery, K. Read and G. Trahern, Cornell Internal Note CSN-22, 1985 (unpublished).

[60] A. Belloni, I.K. Furić, Ch. Paus, Update of $B_{s}^{0}$ event yields using GenTrig, CDF Note 6816.

[61] S. Gadomski, P.K. Sinervo, W. Taylor, W. Trischuk, A combined measurement of the B Meson Fragmentation Fraction Ratio $f_{s} /\left(f_{u}+f_{d}\right)$ Using Run I Inclusive Electron and Dimuon Data, CDF Note 5007, (2000).

[62] T.A. Keaffaber, J.D. Lewis, M.W. Bailey, D. Bortoletto, S. Tkaczyk, A.F. Garfinkel, Measurement of the B Meson Differential Cross Section Using the Exclusive Decay $B \rightarrow J / \psi K$, CDF Note 4911 (to be updated, 2000).

[63] M. Rescigno, B Hadronic Signal Yield in Hadronic Two Track Trigger, talk at the CSL review see: http://www-cdf.fnal.gov/internal/people/links/MarcoRescigno/CSLreview.ppt. 